\title{
EXPLORING THE ROLE OF AMINOCATALYSIS IN THE DEAROMATIZATION AND REGIOSELECTIVITY OF HETEROAROMATIC ALDEHYDES
}

Jorge Dourado, Simarpreet Singh, Rebecca L. Davis*

Chemistry Department, University of Manitoba, 360 Parker Building, 144 Dysart Road, Winnipeg, Manitoba, Canada R3T 2N2

*Corresponding Author: Rebecca L. Davis (ebecca.Davis@umanitoba.ca) ORCID 0000-0002-0679-

6025

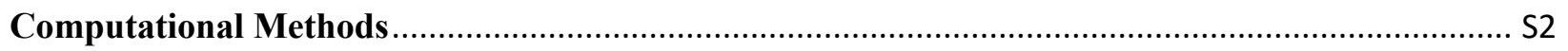

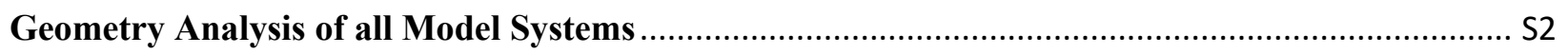

Charge Analysis and Fukui function Isosurfaces for all Model systems ..................................... S11

Hyperhomodesmotic Equations and Orbital Coefficients for the $N$-Methyl systems .................... S14

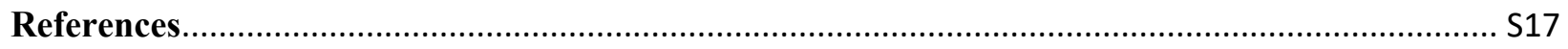

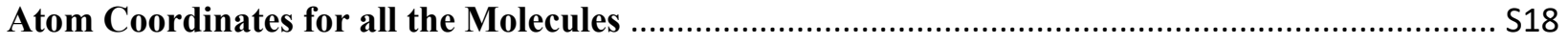




\section{Computational Methods}

All structures were optimized in the gas phase using M06-2X $\mathrm{X}^{1}$ with the double- $\zeta$ split valence 6$31+\mathrm{G}(\mathrm{d}, \mathrm{p})$ basis set $\mathrm{t}^{2,3}$ and vibrational analysis verified that each structure was a minimum. All optimizations were performed in Gaussian 09.e01. ${ }^{4}$ Single point energies were calculated using M06-2X ${ }^{1}$ functional and the correlation-consistent basis set cc-pVTZ ${ }^{5}$ as well as the second order Møller-Plesset perturbation theory $(\mathrm{MP} 2)^{6-9}$ with the cc-pVTZ ${ }^{5}$ basis set. Values discussed in test are free energies calculated at M06-2X/ccpVTZ //M06-2X/6-31+G(d,p). MP2/cc-pVTZ//M06-2X/6-31+G(d,p) energies are included in tables and show the same trends as those calculated with M06-2X/cc-pVTZ //M06-2X/6-31+G(d,p). Electrostatic potential-derived charges were calculated using CHelpG scheme. ${ }^{10}$ The Fukui function describes the change of the electronic density $f(\mathrm{r})$ in a given point with respect to the change in the number of electrons, both calculated at the geometry of the neutral molecule. This approach has been used to predict reactivity in organic molecules. ${ }^{11-14}$ Orbital coefficients were generated with QMForge v2.1. ${ }^{15}$ All 3D figures were generated using VESTA v3.5.5. ${ }^{16}$

\section{Geometry Analysis of all Model Systems}

\section{Model System A}

Table S1. Bond lengths $(\AA)$ and dihedral angles $\left(^{\circ}\right)$ for the aldehydes and enols of model system A.

\begin{tabular}{|c|c|c|c|c|c|c|}
\hline \multirow[b]{2}{*}{ Atom numbers } & \multicolumn{3}{|c|}{$\mathrm{H}^{1 \mathrm{O} 2} \underbrace{3}_{4} \underbrace{7}_{5} x_{6}^{7} 8$} & \multicolumn{3}{|c|}{$\overbrace{2}^{\mathrm{HO}_{2}^{1}} \underbrace{3}_{5} \underbrace{7}_{6}=8$} \\
\hline & $\mathbf{X}=\mathbf{O}$ & $\mathbf{X}=\mathbf{N H}$ & $\mathbf{X}=\mathbf{S}$ & $\mathbf{X}=\mathbf{O}$ & $\mathrm{X}=\mathrm{NH}$ & $\mathbf{X}=\mathbf{S}$ \\
\hline 1,2 & 1.21 & 1.22 & 1.21 & 1.36 & 1.37 & 1.36 \\
\hline 2,3 & 1.46 & 1.45 & 1.46 & 1.34 & 1.34 & 1.34 \\
\hline 3,4 & 1.37 & 1.39 & 1.37 & 1.45 & 1.46 & 1.46 \\
\hline 4,5 & 1.42 & 1.41 & 1.42 & 1.35 & 1.35 & 1.34 \\
\hline 5,6 & 1.37 & 1.39 & 1.37 & 1.46 & 1.47 & 1.46 \\
\hline 6,7 & 1.35 & 1.36 & 1.73 & 1.34 & 1.35 & 1.34 \\
\hline 7,3 & 1.36 & 1.37 & 1.73 & 1.38 & 1.39 & 1.78 \\
\hline
\end{tabular}




\begin{tabular}{|c|c|c|c|c|c|c|}
\hline $\mathbf{1 , 2 , 3 , 4}$ & 180.00 & 180.00 & 180.00 & 1.37 & 1.39 & 1.77 \\
\hline $\mathbf{3 , 4 , 5 , 6}$ & 0.00 & 0.00 & 0.00 & 179.97 & 179.99 & 179.98 \\
\hline $\mathbf{2 , 3 , 7 , 6}$ & 180.00 & 180.00 & 179.99 & 0.01 & 0.00 & 0.00 \\
\hline $\mathbf{N H , 7 , 3 , 4}$ & N.A. & 180.00 & N.A. & 179.96 & 180.00 & 179.99 \\
\hline $\mathbf{N H , 7 , 6 , 5}$ & N.A. & 180.00 & N.A. & N.A. & 180.00 & N.A. \\
\hline
\end{tabular}

Table S2. Bond lengths $(\AA)$ and dihedral angles $\left(^{\circ}\right)$ for the iminium ions and enamines of model system A.

\begin{tabular}{|c|c|c|c|c|c|c|}
\hline Atom numbers & $\mathbf{X}=\mathbf{O}$ & $\mathrm{X}=\mathrm{NH}$ & $\mathrm{X}=\mathrm{S}$ & $\mathbf{X}=\mathbf{O}$ & $\mathbf{X}=\mathbf{N H}$ & $\mathbf{X}=\mathbf{S}$ \\
\hline 1,2 & 1.30 & 1.31 & 1.30 & 1.36 & 1.39 & 1.36 \\
\hline 2,3 & 1.41 & 1.40 & 1.42 & 1.35 & 1.35 & 1.36 \\
\hline 3,4 & 1.38 & 1.41 & 1.39 & 1.43 & 1.46 & 1.45 \\
\hline 4,5 & 1.41 & 1.39 & 1.40 & 1.35 & 1.35 & 1.35 \\
\hline 5,6 & 1.38 & 1.40 & 1.38 & 1.45 & 1.47 & 1.46 \\
\hline 6,8 & 1.35 & 1.35 & 1.72 & 1.34 & 1.35 & 1.34 \\
\hline 6,7 & 1.37 & 1.39 & 1.75 & 1.38 & 1.40 & 1.78 \\
\hline 7,3 & 179.90 & 179.03 & 179.97 & 1.39 & 1.40 & 1.78 \\
\hline $1,2,3,4$ & 0.08 & 0.15 & 0.08 & 179.73 & 175.57 & 179.23 \\
\hline $3,4,5,6$ & 179.56 & 179.38 & 179.17 & 0.00 & 0.60 & 0.11 \\
\hline $2,3,7,6$ & N.A. & 178.38 & N.A. & 179.52 & 175.85 & 177.78 \\
\hline $\mathrm{NH}, 7,3,4$ & N.A. & 178.53 & N.A. & N.A. & 155.38 & N.A. \\
\hline $\mathrm{NH}, 7,6,5$ & 178.93 & 178.69 & 178.86 & N.A. & 155.61 & N.A. \\
\hline $9,1,2,3$ & 1.30 & 1.31 & 1.30 & 7.63 & 48.20 & 19.30 \\
\hline
\end{tabular}




\section{Model System B}

Table S3. Bond lengths $(\AA)$ and dihedral angles $\left(^{\circ}\right)$ for the aldehydes and enols of model system B.

\begin{tabular}{|c|c|c|c|c|c|c|}
\hline \multirow[b]{2}{*}{ Bond } & \multicolumn{3}{|c|}{ 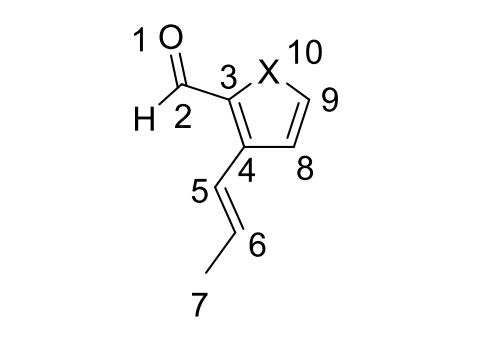 } & \multicolumn{3}{|c|}{ 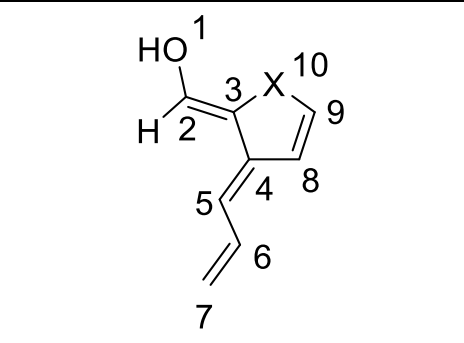 } \\
\hline & $\mathbf{X}=\mathbf{O}$ & $\mathrm{X}=\mathbf{N H}$ & $\mathbf{X}=\mathbf{S}$ & $\mathbf{X}=\mathbf{O}$ & $\mathrm{X}=\mathrm{NH}$ & $\mathbf{X}=\mathbf{S}$ \\
\hline 1,2 & 1.21 & 1.22 & 1.21 & 1.36 & 1.37 & 1.36 \\
\hline 2,3 & 1.46 & 1.45 & 1.46 & 1.34 & 1.34 & 1.34 \\
\hline 3,4 & 1.38 & 1.40 & 1.39 & 1.47 & 1.48 & 1.48 \\
\hline 4,5 & 1.46 & 1.46 & 1.47 & 1.35 & 1.36 & 1.36 \\
\hline 5,6 & 1.34 & 1.34 & 1.34 & 1.45 & 1.47 & 1.45 \\
\hline 6,7 & 1.50 & 1.50 & 1.50 & 1.34 & 1.34 & 1.34 \\
\hline 4,8 & 1.43 & 1.42 & 1.43 & 1.46 & 1.46 & 1.46 \\
\hline 8,9 & 1.36 & 1.38 & 1.37 & 1.34 & 1.35 & 1.34 \\
\hline 9,10 & 1.34 & 1.36 & 1.71 & 1.36 & 1.38 & 1.75 \\
\hline 3,10 & 1.46 & 1.37 & 1.73 & 1.39 & 1.40 & 1.79 \\
\hline $1,2,3,4$ & 179.97 & 179.99 & 179.55 & 179.98 & 179.92 & 179.00 \\
\hline $2,3,4,5$ & 0.00 & 0.01 & 1.38 & 0.01 & 0.06 & 9.93 \\
\hline $3,4,5,6$ & 179.97 & 179.95 & 165.74 & 180.00 & 179.99 & 178.60 \\
\hline $4,5,6,7$ & 179.99 & 179.99 & 179.66 & 180.00 & 179.99 & 179.61 \\
\hline $1,2,3,10$ & 0.03 & 0.00 & 0.86 & 0.02 & 0.06 & 3.69 \\
\hline $3,4,8,9$ & 0.01 & 0.01 & 0.26 & 0.01 & 0.01 & 4.10 \\
\hline $\mathrm{N} H, 10,3,4$ & N.A. & 179.98 & N.A. & N.A. & 179.83 & N.A. \\
\hline $\mathrm{NH}, 10,8,9$ & N.A. & 179.97 & N.A. & N.A. & 179.82 & N.A. \\
\hline
\end{tabular}


Table S4. Bond lengths $(\AA)$ and dihedral angles $\left(^{\circ}\right)$ for the iminium ions and enamines of model system B.

\begin{tabular}{|c|c|c|c|c|c|c|}
\hline \multirow[b]{2}{*}{ Bond } & \multicolumn{3}{|c|}{ 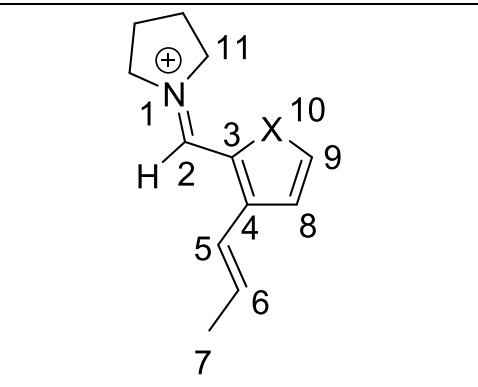 } & \multicolumn{3}{|c|}{ 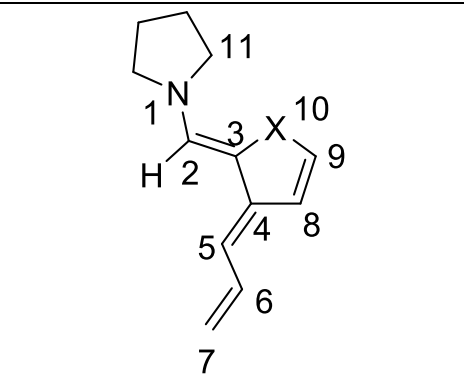 } \\
\hline & $\mathbf{X}=\mathbf{O}$ & $\mathrm{X}=\mathrm{NH}$ & $\mathrm{X}=\mathbf{S}$ & $\mathbf{X}=\mathbf{O}$ & $\mathrm{X}=\mathrm{NH}$ & $\mathbf{X}=\mathbf{S}$ \\
\hline 1,2 & 1.30 & 1.31 & 1.30 & 1.36 & 1.38 & 1.36 \\
\hline 2,3 & 1.41 & 1.40 & 1.41 & 1.35 & 1.35 & 1.36 \\
\hline 3,4 & 1.40 & 1.42 & 1.41 & 1.46 & 1.47 & 1.47 \\
\hline 4,5 & 1.45 & 1.46 & 1.46 & 1.36 & 1.36 & 1.36 \\
\hline 5,6 & 1.34 & 1.34 & 1.34 & 1.45 & 1.45 & 1.45 \\
\hline 6,7 & 1.49 & 1.49 & 1.49 & 1.34 & 1.34 & 1.34 \\
\hline 4,8 & 1.43 & 1.41 & 1.42 & 1.46 & 1.46 & 1.46 \\
\hline 8,9 & 1.36 & 1.39 & 1.37 & 1.34 & 1.35 & 1.34 \\
\hline 9,10 & 1.34 & 1.35 & 1.70 & 1.36 & 1.39 & 1.75 \\
\hline 3,10 & 1.41 & 1.39 & 1.73 & 1.40 & 1.42 & 1.79 \\
\hline $1,2,3,4$ & 179.60 & 177.64 & 178.70 & 179.31 & 176.04 & 177.23 \\
\hline $2,3,4,5$ & 1.49 & 2.71 & 6.13 & 3.10 & 12.75 & 20.13 \\
\hline $3,4,5,6$ & 177.45 & 166.90 & 157.80 & 178.63 & 178.21 & 174.55 \\
\hline $4,5,6,7$ & 179.85 & 179.33 & 178.96 & 179.79 & 179.74 & 179.20 \\
\hline $1,2,3,10$ & 1.73 & 4.66 & 6.79 & 1.38 & 7.46 & 13.06 \\
\hline $3,4,8,9$ & 0.04 & 0.28 & 0.25 & 0.78 & 3.28 & 6.65 \\
\hline $\mathrm{NH}, 10,3,4$ & N.A. & 177.35 & N.A. & N.A. & 148.74 & N.A. \\
\hline $\mathrm{NH}, 10,8,9$ & N.A. & 177.65 & N.A. & N.A. & 146.10 & N.A. \\
\hline $11,1,2,3$ & 0.64 & 2.69 & 2.08 & 4.15 & 46.61 & 27.45 \\
\hline
\end{tabular}




\section{Model System C}

Table S5. Bond lengths $(\AA)$ and dihedral angles $\left(^{\circ}\right)$ for the aldehydes and enols of model system C.

\begin{tabular}{|c|c|c|c|c|c|c|}
\hline \multirow[b]{2}{*}{ Bond } & \multicolumn{3}{|c|}{$\int_{6}^{1}$} & \multicolumn{3}{|c|}{$=\left.\right|_{6} ^{1} \mathrm{OH}$} \\
\hline & $\mathbf{X}=\mathbf{O}$ & $\mathrm{X}=\mathrm{NH}$ & $\mathbf{X}=\mathbf{S}$ & $\mathbf{X}=\mathbf{O}$ & $\mathrm{X}=\mathrm{NH}$ & $\mathbf{X}=\mathbf{S}$ \\
\hline 1,2 & 1.21 & 1.21 & 1.21 & 1.35 & 1.35 & 1.35 \\
\hline 2,3 & 1.46 & 1.46 & 1.47 & 1.35 & 1.35 & 1.35 \\
\hline 3,4 & 1.38 & 1.40 & 1.38 & 1.47 & 1.48 & 1.48 \\
\hline 4,5 & 1.45 & 1.46 & 1.46 & 1.34 & 1.36 & 1.35 \\
\hline 5,6 & 1.34 & 1.34 & 1.34 & 1.45 & 1.45 & 1.45 \\
\hline 6,7 & 1.50 & 1.50 & 1.50 & 1.34 & 1.34 & 1.34 \\
\hline 3,8 & 1.44 & 1.43 & 1.43 & 1.47 & 1.47 & 1.46 \\
\hline 8,9 & 1.35 & 1.37 & 1.36 & 1.34 & 1.35 & 1.34 \\
\hline 9,10 & 1.37 & 1.38 & 1.73 & 1.37 & 1.39 & 1.75 \\
\hline 4,10 & 1.35 & 1.36 & 1.73 & 1.39 & 1.40 & 1.79 \\
\hline $1,2,3,4$ & 179.90 & 179.98 & 180.00 & 179.99 & 179.83 & 180.00 \\
\hline $2,3,4,5$ & 0.03 & 0.00 & 0.08 & 0.06 & 6.23 & 0.01 \\
\hline $3,4,5,6$ & 179.84 & 178.60 & 178.71 & 180.00 & 177.92 & 180.00 \\
\hline $4,5,6,7$ & 179.99 & 179.91 & 179.91 & 180.00 & 177.52 & 179.97 \\
\hline $1,2,3,8$ & 0.09 & 0.10 & 0.10 & 0.04 & 2.15 & 0.00 \\
\hline $10,4,5,6$ & 0.14 & 1.33 & 1.32 & 0.01 & 2.67 & 0.00 \\
\hline $10,4,3,8$ & 0.01 & 0.00 & 0.05 & 0.02 & 3.84 & 0.01 \\
\hline $\mathrm{N} H, 10,4,3$ & N.A. & 179.60 & N.A. & N.A. & 165.45 & N.A. \\
\hline $\mathrm{NH}, 10,9,8$ & N.A. & 179.61 & N.A. & N.A. & 164.52 & N.A. \\
\hline
\end{tabular}


Table S6. Bond lengths $(\AA)$ and dihedral angles $\left(^{\circ}\right)$ for the iminium ions and enamines of model system C.

\begin{tabular}{|c|c|c|c|c|c|c|}
\hline Bond & $\mathrm{X}=\mathbf{O}$ & $\mathbf{X}=\mathbf{N H}$ & $\mathrm{X}=\mathbf{S}$ & $\mathrm{Y}=\mathbf{O}$ & $\mathrm{Y}=\mathrm{NH}$ & $\mathrm{X}=\mathrm{S}$ \\
\hline 1,2 & 1.30 & 1.30 & 1.30 & 1.36 & 1.36 & 1.36 \\
\hline 2,3 & 1.41 & 1.41 & 1.42 & 1.36 & 1.36 & 1.37 \\
\hline 3,4 & 1.40 & 1.38 & 1.41 & 1.47 & 1.47 & 1.47 \\
\hline 4,5 & 1.44 & 1.46 & 1.46 & 1.35 & 1.37 & 1.36 \\
\hline 5,6 & 1.34 & 1.34 & 1.34 & 1.45 & 1.44 & 1.45 \\
\hline 6,7 & 1.49 & 1.49 & 1.49 & 1.34 & 1.35 & 1.34 \\
\hline 3,8 & 1.45 & 1.44 & 1.44 & 1.47 & 1.47 & 1.46 \\
\hline 8,9 & 1.35 & 1.36 & 1.36 & 1.34 & 1.35 & 1.34 \\
\hline 9,10 & 1.37 & 1.38 & 1.73 & 1.37 & 1.39 & 1.76 \\
\hline 4,10 & 1.33 & 1.35 & 1.71 & 1.38 & 1.40 & 1.78 \\
\hline $1,2,3,4$ & 177.29 & 176.99 & 175.13 & 176.95 & 176.46 & 173.00 \\
\hline $2,3,4,5$ & 1.33 & 2.96 & 4.10 & 7.02 & 13.30 & 15.02 \\
\hline $3,4,5,6$ & 179.38 & 153.17 & 153.05 & 178.87 & 175.01 & 177.75 \\
\hline $4,5,6,7$ & 179.89 & 178.25 & 178.43 & 179.63 & 176.16 & 179.34 \\
\hline $1,2,3,8$ & 4.12 & 6.95 & 9.50 & 7.84 & 11.06 & 14.42 \\
\hline $10,4,5,6$ & 1.07 & 27.06 & 28.60 & 2.37 & 6.55 & 5.04 \\
\hline $10,4,3,8$ & 0.16 & 0.37 & 1.28 & 2.22 & 6.09 & 6.24 \\
\hline $\mathrm{N} H, 10,4,3$ & N.A. & 175.29 & N.A. & N.A. & 160.87 & N.A. \\
\hline $\mathrm{NH}, 10,9,8$ & N.A. & 175.33 & N.A. & N.A. & 158.80 & N.A. \\
\hline $11,1,2,3$ & 1.23 & 2.02 & 2.60 & 14.74 & 16.78 & 22.09 \\
\hline
\end{tabular}




\section{Model System D}

Table S7. Bond lengths $(\AA)$ and dihedral angles $\left({ }^{\circ}\right)$ for the aldehydes and enols of model system D.

\begin{tabular}{|c|c|c|c|c|c|c|}
\hline \multirow[b]{2}{*}{ Bond } & \multicolumn{3}{|c|}{ 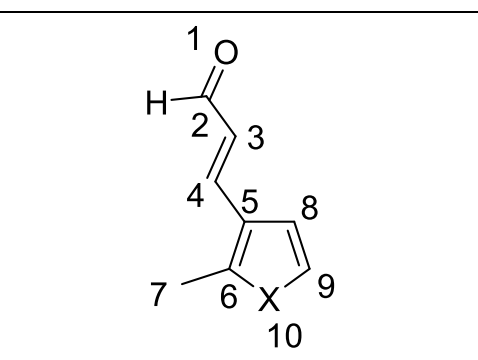 } & \multicolumn{3}{|c|}{ (1) } \\
\hline & $\mathbf{X}=\mathbf{O}$ & $\mathbf{X}=\mathbf{N H}$ & $\mathbf{X}=\mathbf{S}$ & $\mathbf{X}=\mathbf{O}$ & $\mathrm{X}=\mathrm{NH}$ & $\mathbf{X}=\mathbf{S}$ \\
\hline 1,2 & 1.21 & 1.21 & 1.21 & 1.36 & 1.36 & 1.36 \\
\hline 2,3 & 1.47 & 1.46 & 1.47 & 1.34 & 1.34 & 1.34 \\
\hline 3,4 & 1.35 & 1.35 & 1.35 & 1.44 & 1.44 & 1.44 \\
\hline 4,5 & 1.45 & 1.45 & 1.46 & 1.35 & 1.35 & 1.36 \\
\hline 5,6 & 1.37 & 1.39 & 1.38 & 1.48 & 1.49 & 1.49 \\
\hline 6,7 & 1.49 & 1.49 & 1.50 & 1.34 & 1.34 & 1.34 \\
\hline 5,8 & 1.44 & 1.44 & 1.44 & 1.46 & 1.46 & 1.46 \\
\hline 8,9 & 1.35 & 1.37 & 1.36 & 1.34 & 1.35 & 1.34 \\
\hline 9,10 & 1.36 & 1.38 & 1.72 & 1.37 & 1.38 & 1.76 \\
\hline 6,10 & 1.35 & 1.36 & 1.73 & 1.39 & 1.40 & 1.78 \\
\hline $1,2,3,4$ & 179.99 & 179.99 & 180.00 & 179.95 & 179.92 & 179.94 \\
\hline $2,3,4,5$ & 180.00 & 180.00 & 180.00 & 179.95 & 179.93 & 179.90 \\
\hline $3,4,5,6$ & 179.97 & 179.97 & 179.98 & 180.00 & 179.99 & 179.99 \\
\hline $4,5,6,7$ & 0.01 & 0.01 & 0.00 & 0.03 & 0.04 & 0.05 \\
\hline $3,4,5,8$ & 0.04 & 0.03 & 0.02 & 0.01 & 0.01 & 0.03 \\
\hline $10,6,5,4$ & 180.00 & 180.00 & 180.00 & 179.98 & 179.97 & 179.96 \\
\hline $10,6,5,8$ & 0.01 & 0.00 & 0.00 & 0.01 & 0.02 & 0.03 \\
\hline $\mathrm{N} H, 10,6,5$ & N.A. & 180.00 & N.A. & N.A. & 180.00 & N.A. \\
\hline $\mathrm{NH}, 10,9,8$ & N.A. & 180.00 & N.A. & N.A. & 180.00 & N.A. \\
\hline
\end{tabular}


Table S8. Bond lengths $(\AA)$ and dihedral angles $\left(^{\circ}\right)$ for the iminium ions and enamines of model system D.

\begin{tabular}{|c|c|c|c|c|c|c|}
\hline \multirow[b]{2}{*}{ Bond } & & & 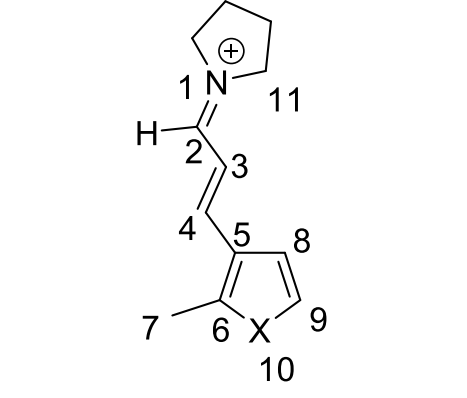 & \multicolumn{3}{|c|}{ (11) } \\
\hline & $\mathbf{X}=\mathbf{O}$ & $\mathbf{X}=\mathbf{N H}$ & $\mathbf{X}=\mathbf{S}$ & $\mathbf{X}=\mathbf{O}$ & $\mathrm{X}=\mathrm{NH}$ & $\mathbf{X}=\mathbf{S}$ \\
\hline 1,2 & 1.30 & 1.31 & 1.30 & 1.36 & 1.36 & 1.35 \\
\hline 2,3 & 1.37 & 1.38 & 1.37 & 1.36 & 1.36 & 1.36 \\
\hline 3,4 & 1.42 & 1.41 & 1.43 & 1.44 & 1.44 & 1.44 \\
\hline 4,5 & 1.39 & 1.41 & 1.40 & 1.36 & 1.36 & 1.36 \\
\hline 5,6 & 1.48 & 1.41 & 1.50 & 1.48 & 1.49 & 1.48 \\
\hline 6,7 & 1.48 & 1.49 & 1.50 & 1.34 & 1.35 & 1.34 \\
\hline 5,8 & 1.45 & 1.44 & 1.44 & 1.46 & 1.46 & 1.46 \\
\hline 8,9 & 1.35 & 1.36 & 1.35 & 1.34 & 1.35 & 1.34 \\
\hline 9,10 & 1.37 & 1.39 & 1.73 & 1.37 & 1.39 & 1.76 \\
\hline 6,10 & 1.33 & 1.35 & 1.71 & 1.39 & 1.40 & 1.79 \\
\hline $1,2,3,4$ & 179.82 & 179.86 & 179.79 & 179.97 & 179.77 & 179.62 \\
\hline $2,3,4,5$ & 179.77 & 179.65 & 179.76 & 179.97 & 179.60 & 179.47 \\
\hline $3,4,5,6$ & 179.95 & 178.68 & 179.76 & 179.97 & 179.84 & 179.95 \\
\hline $4,5,6,7$ & 0.13 & 1.73 & 0.28 & 0.03 & 6.72 & 1.44 \\
\hline $3,4,5,8$ & 0.11 & 1.07 & 0.22 & 0.06 & 1.80 & 0.52 \\
\hline $10,6,5,4$ & 179.96 & 179.76 & 179.94 & 179.99 & 173.98 & 178.75 \\
\hline $10,6,5,8$ & 0.01 & 0.04 & 0.05 & 0.04 & 4.69 & 0.85 \\
\hline $\mathrm{NH}, 10,6,5$ & N.A. & 180.00 & N.A. & N.A. & 162.70 & N.A. \\
\hline $\mathrm{NH}, 10,9,8$ & N.A. & 179.99 & N.A. & N.A. & 161.33 & N.A. \\
\hline $11,1,2,3$ & 0.47 & 0.51 & 0.49 & 0.14 & 0.79 & 0.03 \\
\hline
\end{tabular}




\section{Model System E}

Table S9. Bond lengths $(\AA)$ and dihedral angles $\left(^{\circ}\right)$ for the aldehydes and enols of model system E.

\begin{tabular}{|c|c|c|c|c|c|c|}
\hline & \multicolumn{3}{|c|}{ 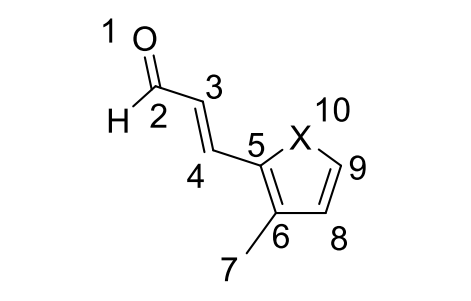 } & \multicolumn{3}{|c|}{$\mathrm{HO}^{1}$} \\
\hline Bond & $\mathbf{X}=\mathbf{O}$ & $\mathbf{X}=\mathbf{N H}$ & $\mathbf{X}=\mathbf{S}$ & $\mathbf{X}=\mathbf{O}$ & $\mathrm{X}=\mathrm{NH}$ & $\mathbf{X}=\mathbf{S}$ \\
\hline 1,2 & 1.21 & 1.21 & 1.21 & 1.35 & 1.36 & 1.35 \\
\hline 2,3 & 1.47 & 1.46 & 1.47 & 1.34 & 1.34 & 1.35 \\
\hline 3,4 & 1.35 & 1.35 & 1.35 & 1.45 & 1.45 & 1.45 \\
\hline 4,5 & 1.44 & 1.44 & 1.45 & 1.34 & 1.35 & 1.35 \\
\hline 5,6 & 1.37 & 1.39 & 1.38 & 1.48 & 1.49 & 1.48 \\
\hline 6,7 & 1.49 & 1.50 & 1.50 & 1.34 & 1.34 & 1.35 \\
\hline 6,8 & 1.43 & 1.42 & 1.43 & 1.47 & 1.47 & 1.47 \\
\hline 8,9 & 1.36 & 1.38 & 1.37 & 1.34 & 1.35 & 1.34 \\
\hline 9,10 & 1.35 & 1.36 & 1.72 & 1.37 & 1.39 & 1.76 \\
\hline 5,10 & 1.36 & 1.38 & 1.74 & 1.39 & 1.41 & 1.78 \\
\hline $1,2,3,4$ & 179.98 & 179.99 & 179.96 & 180.00 & 178.36 & 180.00 \\
\hline $2,3,4,5$ & 179.99 & 180.00 & 179.97 & 179.97 & 173.67 & 179.97 \\
\hline $3,4,5,6$ & 179.99 & 179.99 & 179.57 & 180.00 & 176.72 & 179.99 \\
\hline $4,5,6,7$ & 0.03 & 0.02 & 0.09 & 0.01 & 7.41 & 0.11 \\
\hline $3,4,5,10$ & 0.01 & 0.01 & 0.28 & 0.00 & 3.20 & 0.02 \\
\hline $10,5,6,8$ & 0.01 & 0.01 & 0.04 & 0.00 & 5.65 & 0.06 \\
\hline $7,6,5,10$ & 179.98 & 179.98 & 179.78 & 180.00 & 172.53 & 179.92 \\
\hline $\mathrm{NH}, 10,5,4$ & N.A. & 0.01 & N.A. & N.A. & 28.96 & N.A. \\
\hline $\mathrm{N} H, 10,9,8$ & N.A. & 179.99 & N.A. & N.A. & 150.06 & N.A. \\
\hline
\end{tabular}


Table S10. Bond lengths $(\AA)$ and dihedral angles $\left(^{\circ}\right)$ for the iminium ions and enamines of model system D.

\begin{tabular}{|c|c|c|c|c|c|c|}
\hline Bond & $\mathbf{X}=\mathbf{O}$ & $\mathrm{X}=\mathbf{N H}$ & $\mathbf{X}=\mathbf{S}$ & $\mathbf{X}=\mathbf{O}$ & $\mathrm{X}=\mathrm{NH}$ & $\mathbf{X}=\mathbf{S}$ \\
\hline 1,2 & 1.30 & 1.31 & 1.30 & 1.36 & 1.36 & 1.35 \\
\hline 2,3 & 1.41 & 1.41 & 1.41 & 1.36 & 1.36 & 1.36 \\
\hline 3,4 & 1.37 & 1.38 & 1.37 & 1.44 & 1.44 & 1.44 \\
\hline 4,5 & 1.47 & 1.40 & 1.41 & 1.35 & 1.35 & 1.35 \\
\hline 5,6 & 1.38 & 1.42 & 1.40 & 1.47 & 1.48 & 1.48 \\
\hline 6,7 & 1.49 & 1.50 & 1.50 & 1.35 & 1.35 & 1.35 \\
\hline 6,8 & 1.42 & 1.40 & 1.41 & 1.47 & 1.47 & 1.47 \\
\hline 8,9 & 1.37 & 1.39 & 1.37 & 1.34 & 1.35 & 1.34 \\
\hline 9,10 & 1.34 & 1.35 & 1.71 & 1.36 & 1.39 & 1.75 \\
\hline 5,10 & 1.37 & 1.39 & 1.74 & 1.40 & 1.41 & 1.78 \\
\hline $1,2,3,4$ & 179.87 & 179.89 & 179.72 & 179.63 & 179.56 & 179.49 \\
\hline $2,3,4,5$ & 179.73 & 179.73 & 179.65 & 179.71 & 175.65 & 179.62 \\
\hline $3,4,5,6$ & 179.95 & 179.41 & 179.74 & 179.94 & 177.28 & 179.80 \\
\hline $4,5,6,7$ & 0.04 & 0.36 & 0.24 & 0.02 & 7.91 & 1.04 \\
\hline $3,4,5,10$ & 0.43 & 0.42 & 0.51 & 0.08 & 3.01 & 0.18 \\
\hline $10,5,6,8$ & 0.02 & 0.00 & 0.01 & 0.02 & 5.84 & 0.57 \\
\hline $7,6,5,10$ & 179.95 & 179.56 & 179.76 & 179.96 & 172.34 & 179.31 \\
\hline $\mathrm{NH}, \mathbf{1 0 , 5 , 4}$ & N.A. & 0.47 & N.A. & N.A. & 29.25 & N.A. \\
\hline $\mathrm{NH}, 10,9,8$ & N.A. & 179.64 & N.A. & N.A. & 149.33 & N.A. \\
\hline $11,1,2,3$ & 0.43 & 0.42 & 0.51 & 0.22 & 3.42 & 0.32 \\
\hline
\end{tabular}

\section{Charge Analysis and Fukui function Isosurfaces for all Model systems}

Table S11. ESP charges of trienamines A12-15.

\begin{tabular}{|c|c|c|c|c|}
\hline Carbon & $\begin{array}{c}\mathbf{A 1 2} \\
\left(\mathrm{X}=\mathrm{CH}_{2}\right)\end{array}$ & $\begin{array}{c}\mathbf{A 1 3} \\
(\mathrm{X}=\mathrm{O})\end{array}$ & $\begin{array}{c}\mathbf{A 1 4} \\
(\mathrm{X}=\mathrm{NH})\end{array}$ & $\begin{array}{c}\mathbf{A 1 5} \\
(\mathrm{X}=\mathrm{S})\end{array}$ \\
\hline $\boldsymbol{i p s o}$ & -0.085 & -0.363 & -0.153 & -0.154 \\
\hline $\boldsymbol{\alpha}$ & -0.150 & 0.266 & 0.151 & 0.044 \\
\hline $\boldsymbol{\beta}$ & -0.143 & -0.228 & -0.145 & -0.136 \\
\hline $\boldsymbol{\gamma}$ & -0.211 & -0.221 & -0.220 & 0.249 \\
\hline $\boldsymbol{\delta}$ & 0.138 & 0.425 & 0.459 & 0.356 \\
\hline $\boldsymbol{\varepsilon}$ & $\mathbf{- 0 . 5 6 2}$ & $\mathbf{- 0 . 6 9 1}$ & $\mathbf{- 0 . 7 5 1}$ & $\mathbf{- 0 . 6 2 1}$ \\
\hline
\end{tabular}




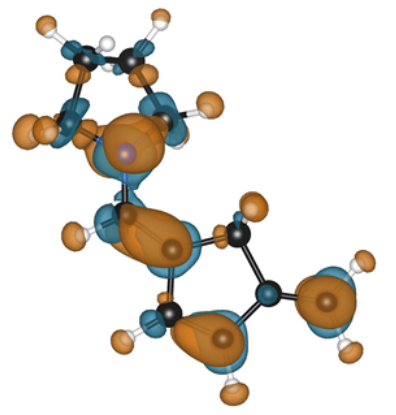

A12

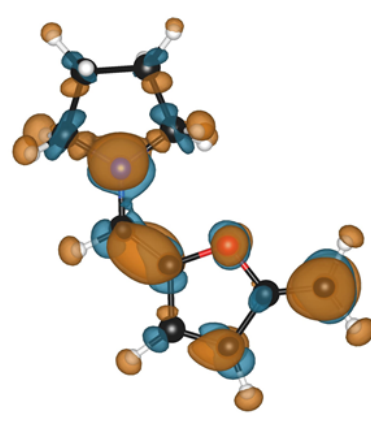

A13

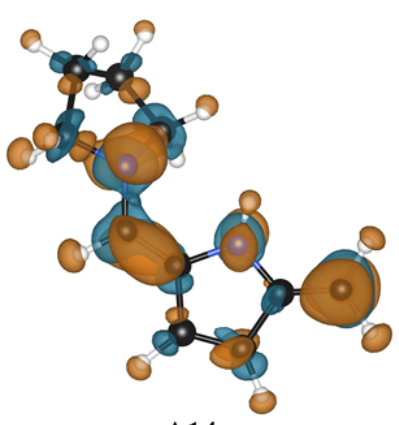

A14

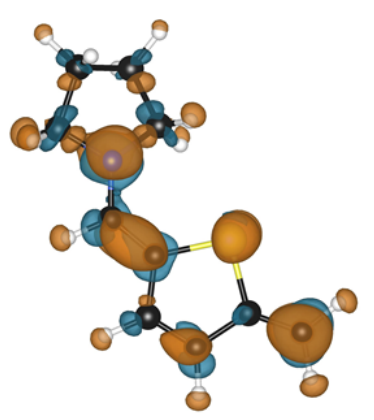

$\mathbf{A 1 5}$

Figure S1. Electrophilic Fukui function $f^{-}$(r) for trienamines A12-15 (isovalue 0.003).

Table S12. ESP charges of trienamines B12-B15.

\begin{tabular}{|c|c|c|c|c|}
\hline Carbon & $\begin{array}{c}\text { B12 } \\
\left(\mathrm{X}=\mathrm{CH}_{2}\right)\end{array}$ & $\begin{array}{c}\text { B13 } \\
(\mathrm{X}=\mathrm{O})\end{array}$ & $\begin{array}{c}\text { B14 } \\
(\mathrm{X}=\mathrm{NH})\end{array}$ & $\begin{array}{c}\text { B15 } \\
(\mathrm{X}=\mathrm{S})\end{array}$ \\
\hline $\boldsymbol{i p s o}$ & -0.033 & -0.297 & -0.109 & -0.091 \\
\hline $\boldsymbol{\alpha}$ & -0.272 & 0.130 & 0.039 & -0.076 \\
\hline $\boldsymbol{\beta}$ & 0.148 & 0.097 & 0.204 & 0.221 \\
\hline $\boldsymbol{\gamma}$ & -0.312 & -0.323 & -0.363 & -0.333 \\
\hline $\boldsymbol{\delta}$ & 0.091 & 0.088 & 0.121 & 0.078 \\
\hline $\boldsymbol{\varepsilon}$ & $\mathbf{- 0 . 4 6 0}$ & $\mathbf{- 0 . 4 8 8}$ & $\mathbf{- 0 . 4 9 1}$ & $\mathbf{- 0 . 4 6 0}$ \\
\hline
\end{tabular}
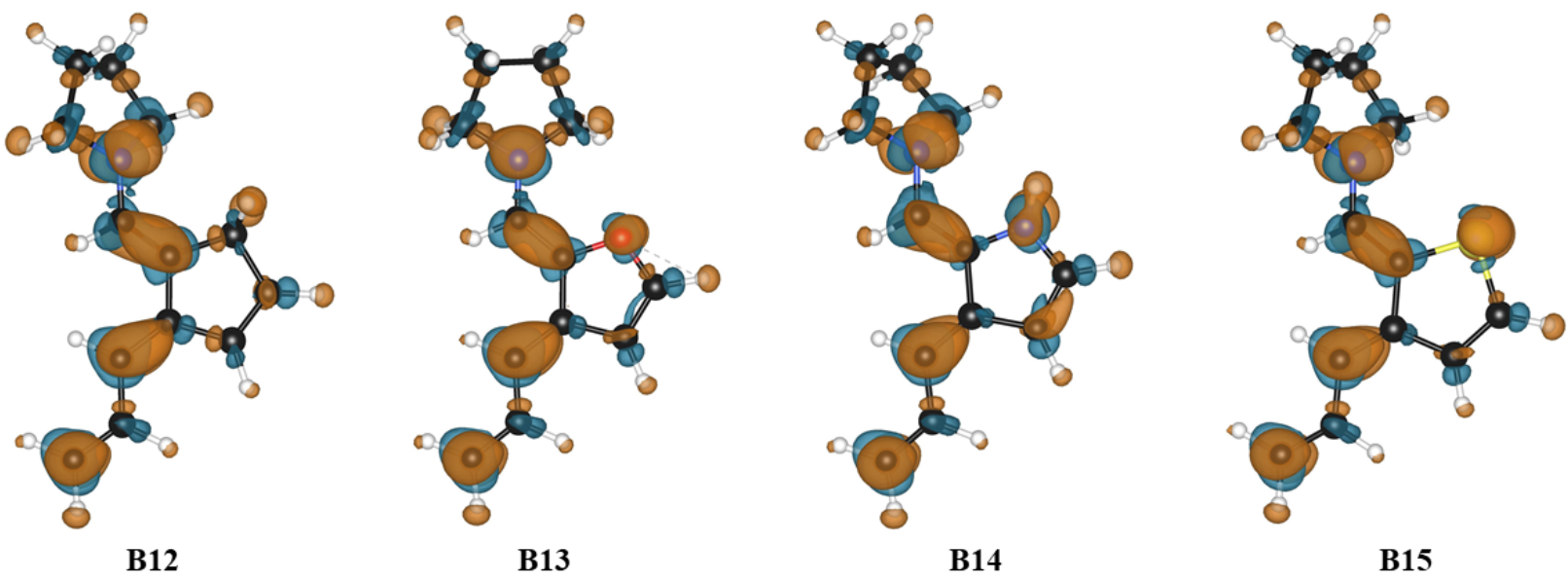

Figure S2. Electrophilic Fukui function $f^{\prime}$ (r) for trienamines B12-15 (isovalue 0.003).

Table S13. ESP charges of trienamines C12-C15.

\begin{tabular}{|c|c|c|c|c|}
\hline Carbon & $\begin{array}{c}\mathbf{C 1 2} \\
\left(\mathrm{X}=\mathrm{CH}_{2}\right)\end{array}$ & $\begin{array}{c}\mathbf{C 1 3} \\
(\mathrm{X}=\mathrm{O})\end{array}$ & $\begin{array}{c}\mathbf{C 1 4} \\
(\mathrm{X}=\mathrm{NH})\end{array}$ & $\begin{array}{c}\mathbf{C 1 5} \\
(\mathrm{X}=\mathrm{S})\end{array}$ \\
\hline $\boldsymbol{i p s o}$ & -0.152 & -0.278 & -0.249 & -0.240 \\
\hline $\boldsymbol{\alpha}$ & 0.019 & 0.107 & 0.137 & 0.167 \\
\hline $\boldsymbol{\beta}$ & -0.143 & 0.189 & 0.137 & -0.024 \\
\hline $\boldsymbol{\gamma}$ & -0.238 & -0.385 & -0.397 & -0.218 \\
\hline $\boldsymbol{\delta}$ & 0.051 & 0.186 & 0.152 & -0.096 \\
\hline $\boldsymbol{\varepsilon}$ & $\mathbf{- 0 . 4 4 3}$ & $\mathbf{- 0 . 5 1 9}$ & $\mathbf{- 0 . 5 2 6}$ & $\mathbf{- 0 . 4 8 6}$ \\
\hline
\end{tabular}




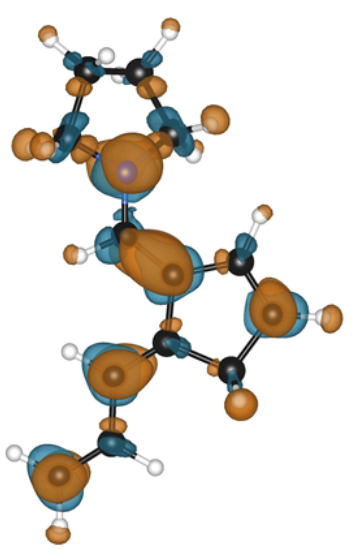

C12

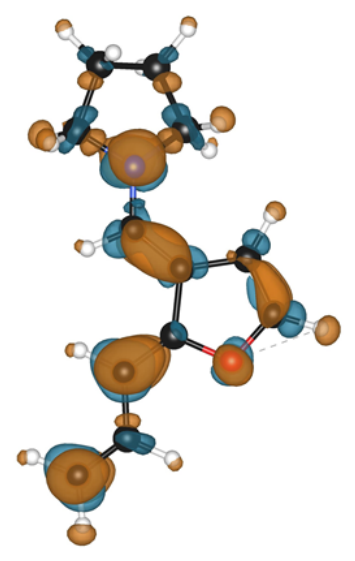

C13

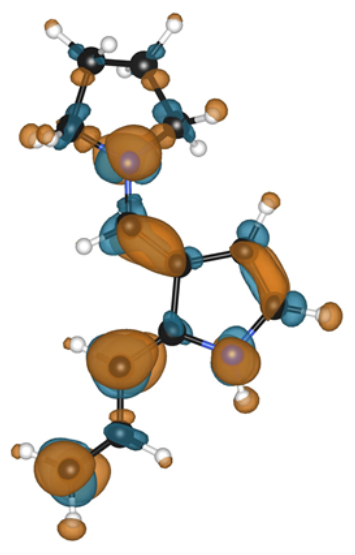

C14

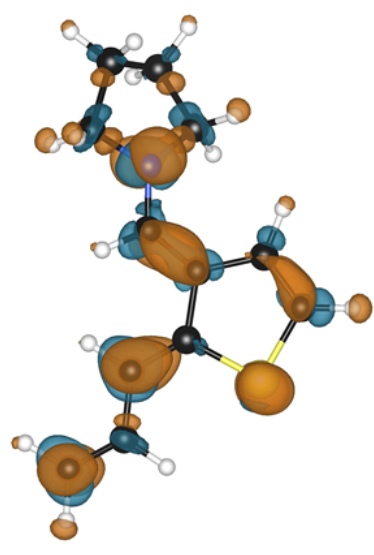

C15

Figure S3. Electrophilic Fukui function $f^{\prime}$ (r) for trienamines C12-15 (isovalue 0.003).

Table S14. ESP charges of trienamines D12-D15.

\begin{tabular}{|c|c|c|c|c|}
\hline Carbon & $\begin{array}{c}\mathbf{D 1 2} \\
\left(\mathrm{X}=\mathrm{CH}_{2}\right)\end{array}$ & $\begin{array}{c}\mathbf{D 1 3} \\
(\mathrm{X}=\mathrm{O})\end{array}$ & $\begin{array}{c}\mathbf{D 1 4} \\
(\mathrm{X}=\mathrm{NH})\end{array}$ & $\begin{array}{c}\text { D15 } \\
(\mathrm{X}=\mathrm{S})\end{array}$ \\
\hline $\boldsymbol{i p s o}$ & -0.159 & -0.173 & -0.131 & -0.107 \\
\hline $\boldsymbol{\alpha}$ & -0.150 & -0.120 & -0.156 & -0.164 \\
\hline $\boldsymbol{\beta}$ & -0.197 & -0.260 & -0.254 & -0.272 \\
\hline $\boldsymbol{\gamma}$ & 0.065 & 0.126 & 0.141 & 0.189 \\
\hline $\boldsymbol{\delta}$ & -0.009 & 0.301 & 0.258 & 0.130 \\
\hline $\boldsymbol{\varepsilon}$ & $\mathbf{- 0 . 4 7 3}$ & $\mathbf{- 0 . 5 9 0}$ & $\mathbf{- 0 . 6 3 5}$ & $\mathbf{- 0 . 5 1 5}$ \\
\hline
\end{tabular}
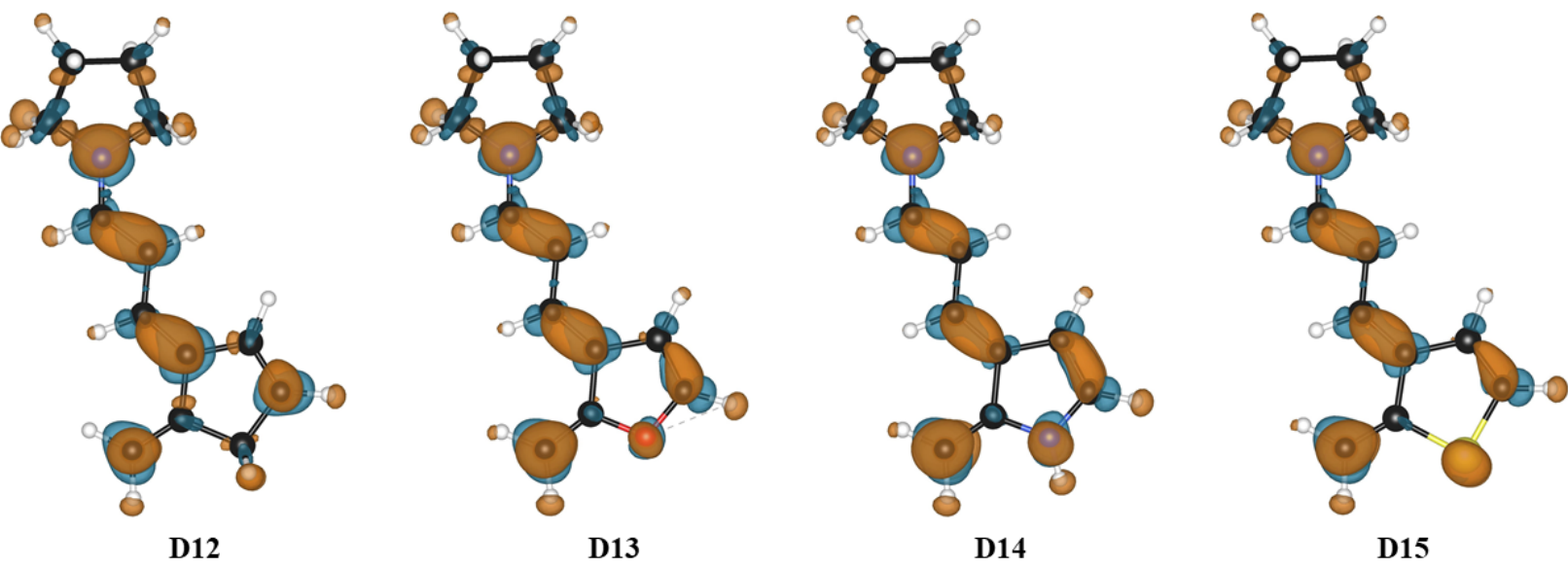

Figure S4. Electrophilic Fukui function $f^{\prime}$ (r) for trienamines D12-15 (isovalue 0.003).

Table S15. ESP charges of trienamines E12-E15.

\begin{tabular}{|c|c|c|c|c|}
\hline Carbon & $\begin{array}{c}\text { E12 } \\
\left(\mathrm{X}=\mathrm{CH}_{2}\right)\end{array}$ & $\begin{array}{c}\text { E13 } \\
(\mathrm{X}=\mathrm{O})\end{array}$ & $\begin{array}{c}\text { E14 } \\
(\mathrm{X}=\mathrm{NH})\end{array}$ & $\begin{array}{c}\text { E15 } \\
(\mathrm{X}=\mathrm{S})\end{array}$ \\
\hline ipso & -0.150 & -0.189 & -0.177 & -0.181 \\
\hline $\boldsymbol{\alpha}$ & -0.179 & -0.059 & -0.102 & -0.162 \\
\hline $\boldsymbol{\beta}$ & -0.130 & -0.269 & -0.227 & -0.089 \\
\hline $\boldsymbol{\gamma}$ & -0.220 & 0.128 & 0.044 & -0.101 \\
\hline $\boldsymbol{\delta}$ & 0.283 & 0.318 & 0.381 & 0.429 \\
\hline $\boldsymbol{\varepsilon}$ & $\mathbf{- 0 . 5 9 0}$ & $\mathbf{- 0 . 6 6 0}$ & $\mathbf{- 0 . 6 5 8}$ & $\mathbf{- 0 . 6 7 6}$ \\
\hline
\end{tabular}



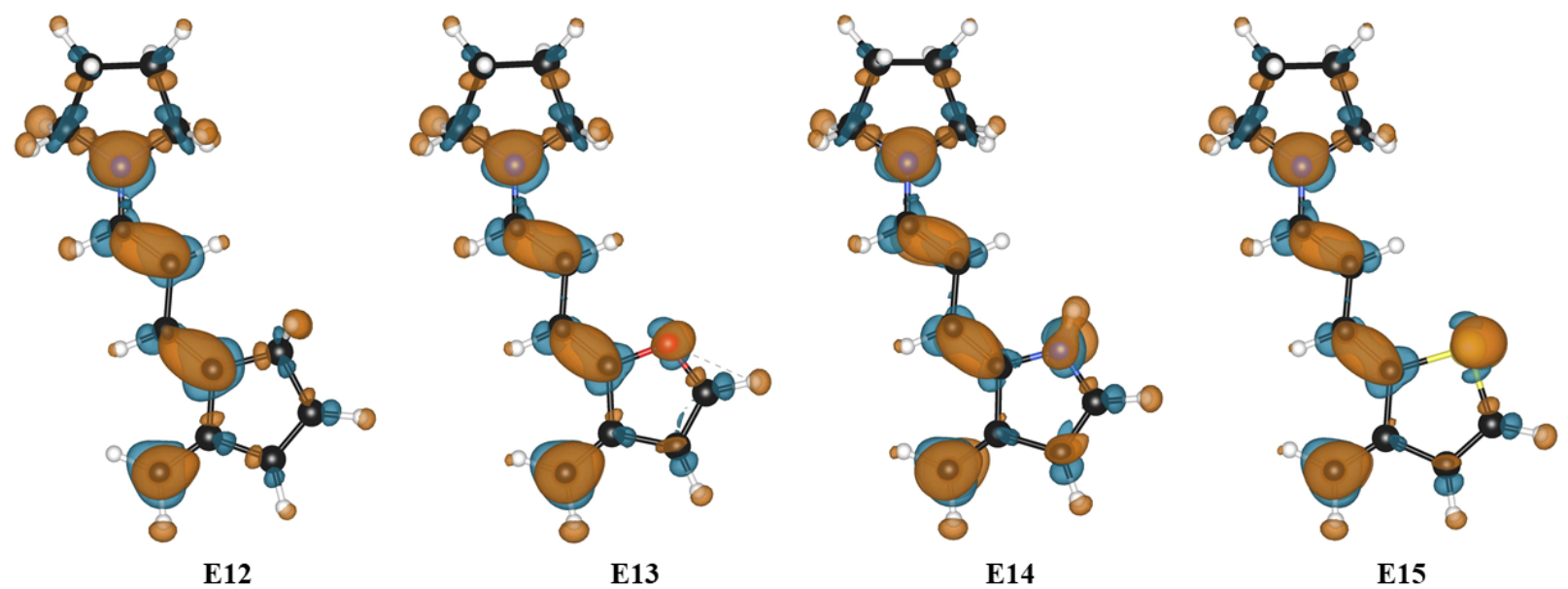

Figure S5. Electrophilic Fukui function $f^{\prime}$ (r) for trienamines E12-15 (isovalue 0.003).

\section{Hyperhomodesmotic Equations and Orbital Coefficients for the $N$-Methyl systems}

Model System A

(1)

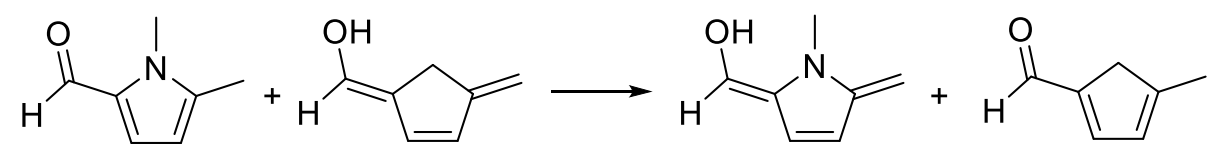

Me-A2

A4

Me-A6

A8

$[0.0]$

19.3

(2)<smiles></smiles><smiles>C=C1C=CC(=CN2CCCC2)C1</smiles>

A12
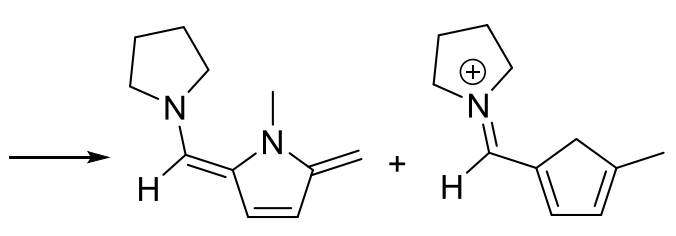

$G_{(\text {eq. } 2 \text { - eq. 1) }}=-7.0$

Me-A10

[0.0]

Me-A14

A16

12.3

Scheme S1 - Hyperhomodesmotic equations for the $N$-methyl derivative of model system A (relative energies in $\mathrm{kcal} / \mathrm{mol}$ ) 


\section{Model System B}

(3)<smiles>C/C=C/c1ccn(C)c1C=O</smiles>

Me-B2<smiles>C=CC=C1C=C(C)CC1=CO</smiles>

B4<smiles>C=CC=C1C=[C+]N(C)C1=CO</smiles>

Me-B6<smiles>CC=CC1=C(C=O)CC=C1</smiles>

B8

[0.0]

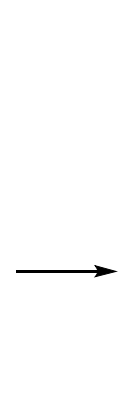

B12<smiles>C=CC=C1C=CCC1=CN1CCCC1</smiles>

Me-B10

[0.0]

19.6<smiles>C=C/C=c1/ccn(C)/c1=C\N1CCCC1</smiles>

Me-B14

$$
G_{(\text {eq. } 4 \text { - eq. 3) }}=-7.7
$$

Scheme S2 - Hyperhomodesmotic equations for the $N$-methyl derivative of model system B (relative energies in $\mathrm{kcal} / \mathrm{mol}$ )

\section{Model System C}

(5)<smiles>C/C=C/C1=C(C=O)C=C[C+]1C</smiles>

$\mathrm{Me}-\mathrm{C2}$

[0.0]<smiles>C=CC=C1CC=CC1=CO</smiles>

C4

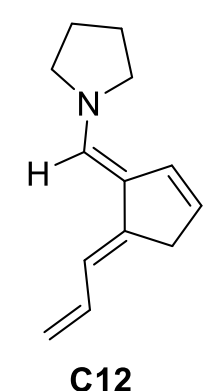<smiles>C=CC=c1c(=C(C)C)ccn1C</smiles>

Me-C6<smiles>CC=CC1=C(C=O)C=CC1</smiles>

C8

(6)<smiles>C/C=C/C1=C(C=[N+]2CCCC2)C=C[CH+]1</smiles>

Me-C10

[0.0]

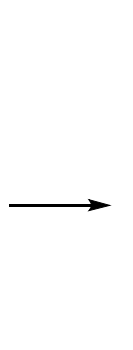

$\mathrm{Me}-\mathrm{C} 14$

15.1

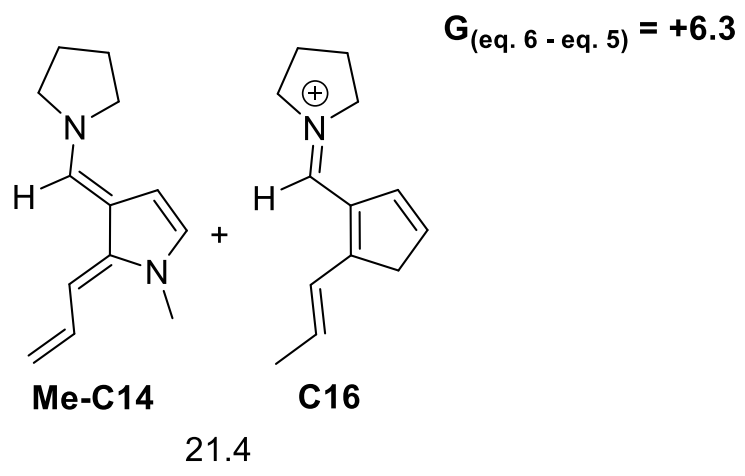

Scheme S3 - Hyperhomodesmotic equations for the $N$-methyl derivative of model system $\mathrm{C}$ (relative energies in $\mathrm{kcal} / \mathrm{mol}$ ) 


\section{Model System D}

(7)<smiles>Cc1c(/C=C/C=O)ccn1C</smiles>

Me_D2

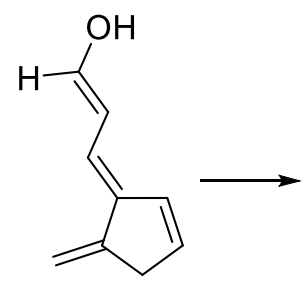

D4<smiles>Cc1c(/C=C/C=[N+]2CCCC2)ccn1C</smiles>

Me-D10

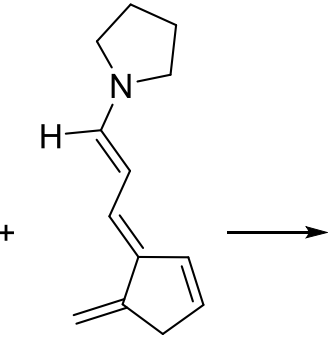

D12<smiles>C=c1/c(=C/C=C/O)ccn1C</smiles>

Me-D6

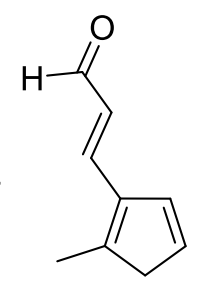

D8

15.1

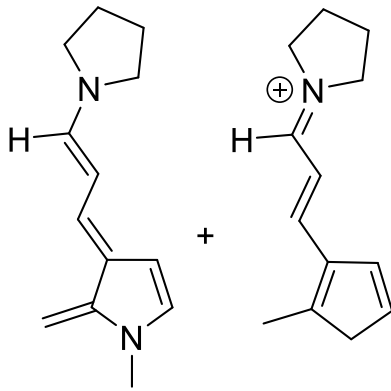

Me-D14 D16

[0.0]

21.3

Scheme S4 - Hyperhomodesmotic equations for the $N$-methyl derivative of model system D (relative energies in $\mathrm{kcal} / \mathrm{mol}$ )

Model System E

(9)<smiles></smiles>

Me-E2<smiles></smiles>

Me-E10<smiles>C=C1C=CCC1=C/C=C/O</smiles>

E4

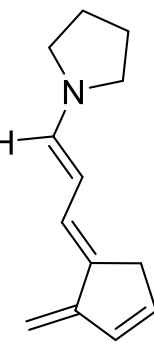

E12

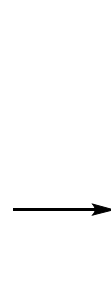<smiles>C=C1C=C[NH+]/C1=C/C=C/O</smiles>

Me-E6

$$
G_{(\text {eq. } 8 \text { - eq. } 7)}=+6.2
$$

[0.0]

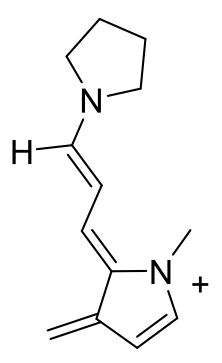

Me-E14<smiles>CC1=C(/C=C/C=O)CC=C1</smiles>

E8<smiles>[C+]1CCCC1</smiles><smiles>CC=N</smiles><smiles>C=CC1=C(C)C=CC1</smiles>

E16

18.5

Scheme S5 - Hyperhomodesmotic equations for the $N$-methyl derivative of model system E (relative energies in $\mathrm{kcal} / \mathrm{mol}$ ) 
Table S16. Orbital coefficients of the HOMO of $N$-Me trienamines.

\begin{tabular}{|c|c|c|c|c|c|}
\hline Carbon & $\begin{array}{c}\text { Model } \\
\text { System A }\end{array}$ & $\begin{array}{c}\text { Model } \\
\text { System B }\end{array}$ & $\begin{array}{c}\text { Model } \\
\text { System C }\end{array}$ & $\begin{array}{c}\text { Model } \\
\text { System D }\end{array}$ & $\begin{array}{c}\text { Model } \\
\text { System E }\end{array}$ \\
\hline $\boldsymbol{i p s o}$ & 18.7 & 14.0 & 10.5 & 8.2 & 9.1 \\
\hline $\boldsymbol{\alpha}$ & 10.3 & 8.3 & 9.4 & 9.2 & 10.2 \\
\hline $\boldsymbol{\beta}$ & 2.8 & 5.2 & 2.8 & 11.9 & 13.3 \\
\hline $\boldsymbol{\gamma}$ & 6.2 & $\mathbf{1 9 . 3}$ & $\mathbf{2 2 . 8}$ & 10.5 & 9.8 \\
\hline $\boldsymbol{\delta}$ & 2.0 & 1.5 & 1.2 & 1.0 & 1.5 \\
\hline $\boldsymbol{\varepsilon}$ & $\mathbf{2 0 . 4}$ & 10.1 & 11.6 & $\mathbf{1 7 . 8}$ & $\mathbf{1 5 . 3}$ \\
\hline
\end{tabular}

Table S17. Comparison of Orbital coefficients of the HOMOs of $N$-H-trienamines and $N$-Me trienamines

\begin{tabular}{|c|c|c|c|c|c|c|c|c|c|c|}
\hline Carbon & $\mathbf{A 1 4}$ & $\begin{array}{c}\text { Me- } \\
\mathbf{A 1 4}\end{array}$ & $\mathbf{B 1 4}$ & $\begin{array}{c}\text { Me- } \\
\mathbf{B 1 4}\end{array}$ & $\mathbf{C 1 4}$ & $\begin{array}{c}\text { Me- } \\
\mathbf{C 1 4}\end{array}$ & $\mathbf{D 1 4}$ & $\begin{array}{c}\text { Me- } \\
\text { D14 }\end{array}$ & $\mathbf{E 1 4}$ & $\begin{array}{c}\text { Me- } \\
\text { E14 }\end{array}$ \\
\hline $\boldsymbol{i p s o}$ & 15.2 & 18.7 & 14.4 & 14.0 & 10.7 & 10.5 & 9.1 & 8.2 & 9.3 & 9.1 \\
\hline $\boldsymbol{\alpha}$ & 8.4 & 10.3 & 8.1 & 8.3 & 10.2 & 9.4 & 12.9 & 9.2 & 11.4 & 10.2 \\
\hline $\boldsymbol{\beta}$ & 3.3 & 2.8 & 4.8 & 5.2 & 3.0 & 2.8 & 11.2 & 11.9 & 13.3 & 13.3 \\
\hline $\boldsymbol{\gamma}$ & 5.6 & 6.2 & $\mathbf{1 9 . 4}$ & $\mathbf{1 9 . 3}$ & $\mathbf{2 2 . 6}$ & $\mathbf{2 2 . 8}$ & 13.9 & 10.5 & 11.1 & 9.8 \\
\hline $\boldsymbol{\delta}$ & 3.7 & 2.0 & 1.4 & 1.5 & 1.2 & 1.2 & 1.2 & 1.0 & 1.7 & 1.5 \\
\hline $\boldsymbol{\varepsilon}$ & $\mathbf{2 0 . 6}$ & $\mathbf{2 0 . 4}$ & 10.1 & 10.1 & 11.3 & 11.6 & $\mathbf{1 4 . 8}$ & $\mathbf{1 7 . 8}$ & $\mathbf{1 5 . 3}$ & $\mathbf{1 5 . 3}$ \\
\hline
\end{tabular}

\section{References}

(1) Zhao, Y.; Truhlar, D. G. The M06 Suite of Density Functionals for Main Group Thermochemistry, Thermochemical Kinetics, Noncovalent Interactions, Excited States, and Transition Elements: Two New Functionals and Systematic Testing of Four M06-Class Functionals and 12 Other Function. Theor. Chem. Acc. 2008, 120 (1-3), 215-241. https://doi.org/10.1007/s00214-007-0310$\mathrm{x}$.

(2) Petersson, G. A.; Bennett, A.; Tensfeldt, T. G.; Al-Laham, M. A.; Shirley, W. A.; Mantzaris, J. A Complete Basis Set Model Chemistry. I. The Total Energies of Closed-Shell Atoms and Hydrides of the First-Row Elements. J. Chem. Phys. 1988, 89 (4), 2193-2218. https://doi.org/10.1063/1.455064.

(3) Petersson, G. A.; Al-Laham, M. A. A Complete Basis Set Model Chemistry. II. Open-Shell Systems and the Total Energies of the First-Row Atoms. J. Chem. Phys. 1991, 94 (9), 6081-6090. https://doi.org/10.1063/1.460447.

(4) Frisch, M. J.; Trucks, G. W.; Schlegel, H. B.; Scuseria, G. E.; Robb, M. a.; Cheeseman, J. R.; Scalmani, G.; Barone, V.; Petersson, G. a.; Nakatsuji, H.; Li, X.; Caricato, M.; Marenich, a. V.; Bloino, J.; Janesko, B. G.; Gomperts, R.; Mennucci, B.; Hratchian, H. P.; Ortiz, J. V.; Izmaylov, a. F.; Sonnenberg, J. L.; Williams; Ding, F.; Lipparini, F.; Egidi, F.; Goings, J.; Peng, B.; Petrone, A.; Henderson, T.; Ranasinghe, D.; Zakrzewski, V. G.; Gao, J.; Rega, N.; Zheng, G.; Liang, W.; Hada, M.; Ehara, M.; Toyota, K.; Fukuda, R.; Hasegawa, J.; Ishida, M.; Nakajima, T.; Honda, Y.; Kitao, O.; Nakai, H.; Vreven, T.; Throssell, K.; Montgomery Jr., J. a.; Peralta, J. E.; Ogliaro, F.; Bearpark, M. J.; Heyd, J. J.; Brothers, E. N.; Kudin, K. N.; Staroverov, V. N.; Keith, T. a.; Kobayashi, R.; Normand, J.; Raghavachari, K.; Rendell, a. P.; Burant, J. C.; Iyengar, S. S.; Tomasi, J.; Cossi, M.; Millam, J. M.; Klene, M.; Adamo, C.; Cammi, R.; Ochterski, J. W.; Martin, R. L.; Morokuma, K.; Farkas, O.; Foresman, J. B.; Fox, D. J. G09. 2009, p Gaussian 09, Gaussian, 
Inc., Wallin.

(5) Kendall, R. A.; Jr., T. H. D.; Harrison, R. J. Electron Affinities of the First-row Atoms Revisited. Systematic Basis Sets and Wave Functions. J. Chem. Phys. 1992, 96 (9), 6796.

https://doi.org/10.1063/1.462569.

(6) Head-Gordon, M.; Pople, J. A.; Frisch, M. J. MP2 Energy Evaluation by Direct Methods. Chem. Phys. Lett. 1988, 153 (6), 503-506. https://doi.org/10.1016/0009-2614(88)85250-3.

(7) Sæbø, S.; Almlöf, J. Avoiding the Integral Storage Bottleneck in LCAO Calculations of Electron Correlation. Chem. Phys. Lett. 1989, 154 (1), 83-89. https://doi.org/10.1016/0009-2614(89)874421.

(8) Frisch, M. J.; Head-Gordon, M.; Pople, J. A. A Direct MP2 Gradient Method. Chem. Phys. Lett. 1990, 166 (3), 275-280. https://doi.org/10.1016/0009-2614(90)80029-D.

(9) Head-Gordon, M.; Head-Gordon, T. Analytic MP2 Frequencies without Fifth-Order Storage. Theory and Application to Bifurcated Hydrogen Bonds in the Water Hexamer. Chem. Phys. Lett. 1994, 220 (1-2), 122-128. https://doi.org/10.1016/0009-2614(94)00116-2.

(10) Breneman, C. M.; Wiberg, K. B. Determining Atom-Centered Monopoles from Molecular Electrostatic Potentials. The Need for High Sampling Density in Formamide Conformational Analysis. J. Comput. Chem. 1990, 11 (3), 361-373. https://doi.org/10.1002/JCC.540110311.

(11) Parr, R. G.; Yang, W. Density Functional Approach to the Frontier-Electron Theory of Chemical Reactivity. J. Am. Chem. Soc. 1984, 106 (14), 4049-4050. https://doi.org/10.1021/JA00326A036.

(12) Geerlings, P.; Proft, F. De; Langenaeker, W. Conceptual Density Functional Theory. Chem. Rev. 2003, 103 (5), 1793-1873. https://doi.org/10.1021/CR990029P.

(13) Roy, R. K. Stockholders Charge Partitioning Technique. A Reliable Electron Population Analysis Scheme to Predict Intramolecular Reactivity Sequence. J. Phys. Chem. A 2003, 107 (48), 1042810434. https://doi.org/10.1021/JP035848Z.

(14) Liu, S.; Rong, C.; Lu, T. Information Conservation Principle Determines Electrophilicity, Nucleophilicity, and Regioselectivity. J. Phys. Chem. A 2014, 118 (20), 3698-3704. https://doi.org/10.1021/JP5032702.

(15) Tenderholt, A. L. QMForge, version 2.1 https://sourceforge.net/projects/qmforge/files/qmforge/QMForge-2.1/\%0A.

(16) Momma, K.; Izumi, F. VESTA 3 for Three-Dimensional Visualization of Crystal, Volumetric and Morphology Data. J. Appl. Crystallogr. 2011, 44 (6), 1272-1276.

https://doi.org/10.1107/S0021889811038970.

\section{Atom Coordinates for all the Molecules}

\section{Aldehyde A1}




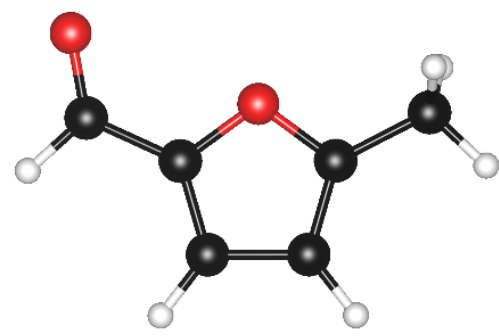

HF $(M 062 X / 6-31+G(d, p))=-382.5277955$ Hartrees

Imaginary Frequencies: none found

Zero-point correction $=0.108322$ (Hartree/Particle)

Thermal correction $=0.076441$ Hartrees

Coordinates from last standard orientation:

\begin{tabular}{|c|c|c|c|c|c|}
\hline \multirow{2}{*}{$\begin{array}{l}\text { Center } \\
\text { Number }\end{array}$} & \multirow{2}{*}{$\begin{array}{l}\text { Atomic } \\
\text { Number }\end{array}$} & \multirow{2}{*}{$\begin{array}{c}\text { Atomic } \\
\text { Type }\end{array}$} & \multicolumn{3}{|c|}{ Coordinates (Angstroms) } \\
\hline & & & $\mathrm{x}$ & $\mathrm{Y}$ & Z \\
\hline & & & 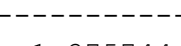 & ---------- & ---------- \\
\hline 1 & 6 & 0 & 1.375744 & -0.126055 & -0.000039 \\
\hline 2 & 6 & 0 & 1.299606 & 1.240881 & -0.000011 \\
\hline 3 & 1 & 0 & 2.137053 & 1.922463 & 0.000080 \\
\hline 4 & 6 & 0 & -0.088014 & 1.560053 & 0.000004 \\
\hline 5 & 1 & 0 & -0.543457 & 2.540475 & 0.000096 \\
\hline 6 & 6 & 0 & -0.754104 & 0.364370 & -0.000024 \\
\hline 7 & 8 & 0 & 0.137251 & -0.661068 & -0.000044 \\
\hline 8 & 6 & 0 & -2.181796 & 0.069935 & 0.000005 \\
\hline 9 & 1 & 0 & -2.818917 & 0.976375 & 0.000020 \\
\hline 10 & 6 & 0 & 2.514381 & -1.080950 & 0.000034 \\
\hline 11 & 1 & 0 & 2.478849 & -1.722943 & -0.884593 \\
\hline 12 & 1 & 0 & 3.457153 & -0.532319 & 0.000007 \\
\hline 13 & 1 & 0 & 2.478872 & -1.722851 & 0.884728 \\
\hline 14 & 8 & 0 & -2.660308 & -1.042757 & 0.000025 \\
\hline
\end{tabular}

\section{Aldehyde A2}

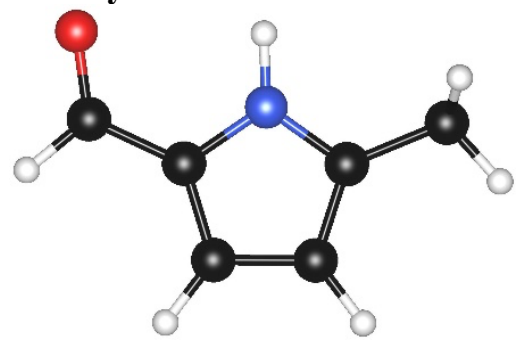

HF $(M 062 X / 6-31+G(d, p))=-362.6872371$ Hartrees

Imaginary Frequencies: none found

Zero-point correction $=0.121432$ (Hartree/Particle)

Thermal correction $=0.089606$ Hartrees

Coordinates from last standard orientation:

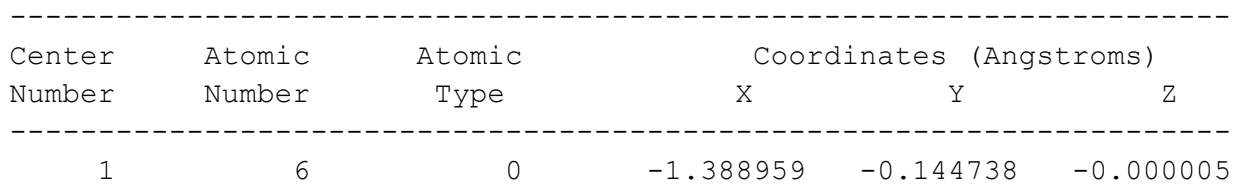




\begin{tabular}{|c|c|c|c|c|c|}
\hline 2 & 6 & 0 & -1.289247 & 1.241453 & 0.000009 \\
\hline 3 & 6 & 0 & 0.083412 & 1.572549 & 0.000000 \\
\hline 4 & 6 & 0 & 0.790787 & 0.379993 & -0.000020 \\
\hline 5 & 7 & 0 & -0.125513 & -0.644201 & -0.000021 \\
\hline 6 & 1 & 0 & 0.146813 & -1.618146 & -0.000017 \\
\hline 7 & 1 & 0 & -2.127298 & 1.923694 & 0.000026 \\
\hline 8 & 1 & 0 & 0.520001 & 2.561822 & -0.000006 \\
\hline 9 & 6 & 0 & -2.593252 & -1.028401 & 0.000009 \\
\hline 10 & 1 & 0 & -2.616925 & -1.670573 & -0.886035 \\
\hline 11 & 1 & 0 & -2.617038 & -1.670398 & 0.886175 \\
\hline 12 & 1 & 0 & -3.497466 & -0.417921 & -0.000107 \\
\hline 13 & 6 & 0 & 2.211180 & 0.096703 & 0.000004 \\
\hline 14 & 1 & 0 & 2.875750 & 0.980476 & 0.000014 \\
\hline 15 & 8 & 0 & 2.663903 & -1.035612 & 0.000014 \\
\hline
\end{tabular}

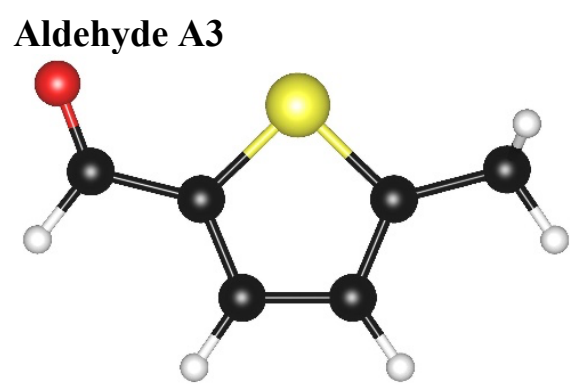

HF $(M 062 X / 6-31+G(d, p))=-705.4955175$ Hartrees

Imaginary Frequencies: none found

Zero-point correction $=0.104736$ (Hartree/Particle)

Thermal correction $=0.070700$ Hartrees

Coordinates from last standard orientation:

\begin{tabular}{|c|c|c|c|c|c|}
\hline \multirow{2}{*}{$\begin{array}{l}\text { Center } \\
\text { Number }\end{array}$} & \multirow{2}{*}{$\begin{array}{l}\text { Atomic } \\
\text { Number }\end{array}$} & \multirow{2}{*}{$\begin{array}{l}\text { Atomic } \\
\text { Type }\end{array}$} & \multicolumn{3}{|c|}{ Coordinates (Angstroms) } \\
\hline & & & $X$ & $Y$ & Z \\
\hline 1 & 6 & 0 & -0.904114 & 0.406018 & 0.000078 \\
\hline 2 & 6 & 0 & -0.180906 & 1.573719 & 0.000046 \\
\hline 3 & 6 & 0 & 1.220542 & 1.346593 & -0.000070 \\
\hline 4 & 6 & 0 & 1.543305 & 0.011991 & -0.000086 \\
\hline 5 & 1 & 0 & -0.646691 & 2.553622 & 0.000110 \\
\hline 6 & 1 & 0 & 1.968404 & 2.131347 & -0.000133 \\
\hline 7 & 16 & 0 & 0.129805 & -0.982765 & -0.000004 \\
\hline 8 & 6 & 0 & 2.913383 & -0.593874 & 0.000039 \\
\hline 9 & 1 & 0 & 3.072886 & -1.218606 & -0.883578 \\
\hline 10 & 1 & 0 & 3.664247 & 0.199244 & -0.001502 \\
\hline 11 & 1 & 0 & 3.073792 & -1.215983 & 0.885357 \\
\hline 12 & 6 & 0 & -2.359849 & 0.283404 & 0.000007 \\
\hline 13 & 1 & 0 & -2.896894 & 1.253285 & -0.000024 \\
\hline 14 & 8 & 0 & -2.963349 & -0.768221 & -0.000030 \\
\hline
\end{tabular}

\section{Enol A4}




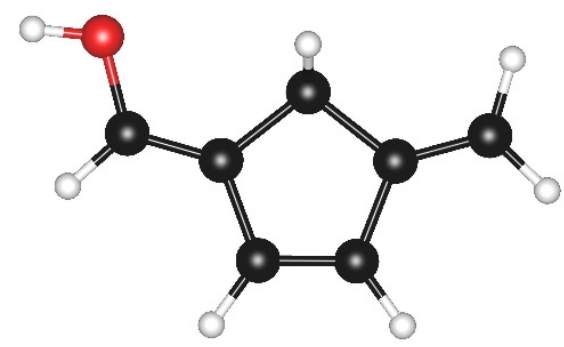

HF $(M 062 X / 6-31+G(d, p))=-346.5931717$ Hartrees

Imaginary Frequencies: none found

Zero-point correction $=0.131596$ (Hartree/Particle)

Thermal correction $=0.099574$ Hartrees

Coordinates from last standard orientation:

\begin{tabular}{|c|c|c|c|c|c|}
\hline \multirow{2}{*}{$\begin{array}{l}\text { Center } \\
\text { Number }\end{array}$} & \multirow{2}{*}{$\begin{array}{l}\text { Atomic } \\
\text { Number }\end{array}$} & \multirow{2}{*}{$\begin{array}{c}\text { Atomic } \\
\text { Type }\end{array}$} & \multicolumn{3}{|c|}{ Coordinates (Angstroms) } \\
\hline & & & $\mathrm{x}$ & Y & z \\
\hline 1 & 6 & 0 & 1.583220 & -0.291727 & -0.000109 \\
\hline 2 & 6 & 0 & 1.439401 & 1.168394 & 0.000162 \\
\hline 3 & 1 & 0 & 2.284272 & 1.847830 & 0.000309 \\
\hline 4 & 6 & 0 & 0.138321 & 1.523998 & 0.000210 \\
\hline 5 & 1 & 0 & -0.240401 & 2.540783 & 0.000556 \\
\hline 6 & 6 & 0 & -0.719189 & 0.343465 & -0.000336 \\
\hline 7 & 6 & 0 & -2.055285 & 0.336594 & -0.000431 \\
\hline 8 & 1 & 0 & -2.644403 & 1.250420 & -0.000299 \\
\hline 9 & 6 & 0 & 2.732576 & -0.977473 & -0.000353 \\
\hline 10 & 1 & 0 & 2.745972 & -2.062922 & -0.000708 \\
\hline 11 & 1 & 0 & 3.689954 & -0.465487 & -0.000488 \\
\hline 12 & 8 & 0 & -2.740394 & -0.846393 & 0.002435 \\
\hline 13 & 1 & 0 & -3.688099 & -0.685655 & -0.014639 \\
\hline 14 & 6 & 0 & 0.177203 & -0.877822 & 0.000056 \\
\hline 15 & 1 & 0 & -0.000924 & -1.503524 & -0.881060 \\
\hline 16 & 1 & 0 & -0.000702 & -1.502873 & 0.881646 \\
\hline
\end{tabular}

\section{Enol A5}

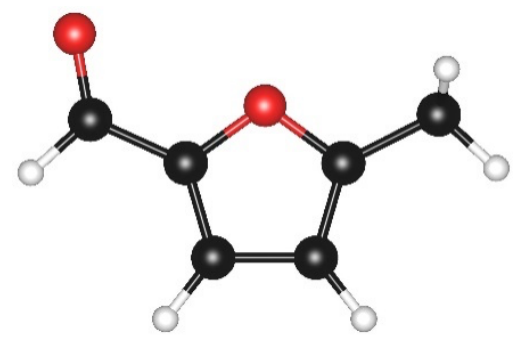

HF $(M 062 X / 6-31+G(d, p))=-382.4882319$ Hartrees

Imaginary Frequencies: none found

Zero-point correction $=0.107953$ (Hartree/Particle)

Thermal correction $=0.076513$ Hartrees

Coordinates from last standard orientation:

Center Atomic Atomic Coordinates (Angstroms)




\begin{tabular}{|c|c|c|c|c|c|}
\hline Number & Number & Type & $\mathrm{x}$ & Y & Z \\
\hline 1 & 6 & 0 & 1.472947 & -0.310612 & 0.000145 \\
\hline 2 & 6 & 0 & 1.470349 & 1.150388 & -0.000030 \\
\hline 3 & 1 & 0 & 2.367223 & 1.753900 & 0.000023 \\
\hline 4 & 6 & 0 & 0.190456 & 1.564747 & -0.000096 \\
\hline 5 & 1 & 0 & -0.182181 & 2.580352 & -0.000005 \\
\hline 6 & 6 & 0 & -0.652523 & 0.383111 & 0.000329 \\
\hline 7 & 8 & 0 & 0.157017 & -0.727622 & 0.000465 \\
\hline 8 & 6 & 0 & -1.989768 & 0.295686 & -0.000219 \\
\hline 9 & 1 & 0 & -2.589699 & 1.199198 & -0.000565 \\
\hline 10 & 6 & 0 & 2.487729 & -1.181719 & -0.000218 \\
\hline 11 & 1 & 0 & 2.304721 & -2.248351 & -0.000307 \\
\hline 12 & 1 & 0 & 3.506223 & -0.816782 & -0.000947 \\
\hline 13 & 8 & 0 & -2.620485 & -0.909123 & -0.000267 \\
\hline 14 & 1 & 0 & -3.573680 & -0.783965 & 0.000745 \\
\hline
\end{tabular}

\section{Enol A6}

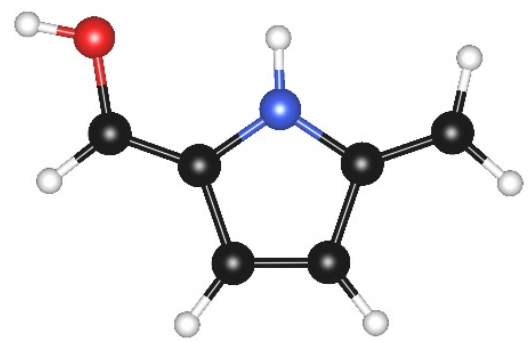

HF $(M 062 X / 6-31+G(d, p))=-362.6370567$ Hartrees

Imaginary Frequencies: none found

Zero-point correction $=0.119909$ (Hartree/Particle)

Thermal correction $=0.087709$ Hartrees

\section{Coordinates from last standard orientation:}

\begin{tabular}{|c|c|c|c|c|c|}
\hline \multirow{2}{*}{$\begin{array}{l}\text { Center } \\
\text { Number }\end{array}$} & \multirow{2}{*}{$\begin{array}{l}\text { Atomic } \\
\text { Number }\end{array}$} & \multirow{2}{*}{$\begin{array}{c}\text { Atomic } \\
\text { Type }\end{array}$} & \multicolumn{3}{|c|}{ Coordinates (Angstroms) } \\
\hline & & & $\mathrm{x}$ & $\mathrm{Y}$ & z \\
\hline 1 & 6 & 0 & -1.503442 & -0.316760 & 0.000030 \\
\hline 2 & 6 & 0 & -1.458566 & 1.152729 & 0.000066 \\
\hline 3 & 1 & 0 & -2.346119 & 1.771075 & 0.000128 \\
\hline 4 & 6 & 0 & -0.175706 & 1.562136 & 0.000020 \\
\hline 5 & 1 & 0 & 0.192039 & 2.579752 & 0.000036 \\
\hline 6 & 6 & 0 & 0.687264 & 0.385075 & -0.000082 \\
\hline 7 & 6 & 0 & 2.028144 & 0.325937 & -0.000062 \\
\hline 8 & 1 & 0 & 2.646147 & 1.215280 & -0.000007 \\
\hline 9 & 6 & 0 & -2.589984 & -1.111325 & 0.000050 \\
\hline 10 & 1 & 0 & -2.509377 & -2.191917 & 0.000008 \\
\hline 11 & 1 & 0 & -3.578034 & -0.670466 & 0.000122 \\
\hline 12 & 8 & 0 & 2.637654 & -0.904564 & 0.000145 \\
\hline 13 & 1 & 0 & 3.593640 & -0.806371 & -0.001082 \\
\hline 14 & 7 & 0 & -0.168551 & -0.711780 & -0.000055 \\
\hline 15 & 1 & 0 & 0.154075 & -1.665137 & -0.000119 \\
\hline
\end{tabular}




\section{Enol A7}

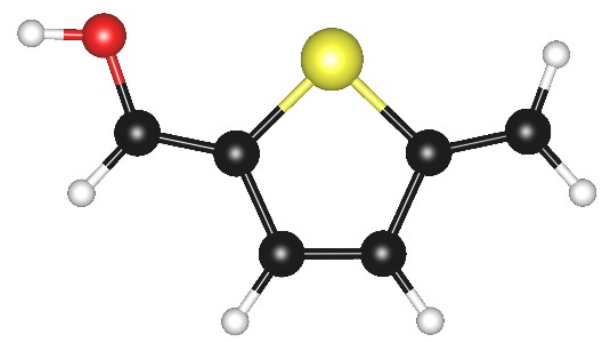

HF $(M 062 X / 6-31+G(d, p))=-705.4574365$ Hartrees

Imaginary Frequencies: none found

Zero-point correction $=0.104529$ (Hartree/Particle)

Thermal correction $=0.071959$ Hartrees

Coordinates from last standard orientation:

\begin{tabular}{|c|c|c|c|c|c|}
\hline \multirow{2}{*}{$\begin{array}{l}\text { Center } \\
\text { Number }\end{array}$} & \multirow{2}{*}{$\begin{array}{l}\text { Atomic } \\
\text { Number }\end{array}$} & \multirow{2}{*}{$\begin{array}{c}\text { Atomic } \\
\text { Type }\end{array}$} & \multicolumn{3}{|c|}{ Coordinates (Angstroms) } \\
\hline & & & $\mathrm{x}$ & Y & Z \\
\hline 1 & 6 & 0 & -1.674854 & -0.112802 & 0.000076 \\
\hline 2 & 6 & 0 & -1.373476 & 1.319149 & 0.000137 \\
\hline 3 & 1 & 0 & -2.174042 & 2.050523 & 0.000240 \\
\hline 4 & 6 & 0 & -0.061874 & 1.613624 & 0.000068 \\
\hline 5 & 1 & 0 & 0.346517 & 2.618870 & 0.000107 \\
\hline 6 & 6 & 0 & 0.809543 & 0.448104 & -0.000078 \\
\hline 7 & 6 & 0 & 2.149281 & 0.467685 & -0.000068 \\
\hline 8 & 1 & 0 & 2.700532 & 1.404107 & -0.000018 \\
\hline 9 & 6 & 0 & -2.894566 & -0.670051 & 0.000122 \\
\hline 10 & 1 & 0 & -3.039977 & -1.744046 & 0.000063 \\
\hline 11 & 1 & 0 & -3.776512 & -0.038367 & 0.000224 \\
\hline 12 & 8 & 0 & 2.859943 & -0.691066 & 0.000051 \\
\hline 13 & 1 & 0 & 3.804831 & -0.512421 & -0.000865 \\
\hline 14 & 16 & 0 & -0.154076 & -1.040274 & -0.000106 \\
\hline
\end{tabular}

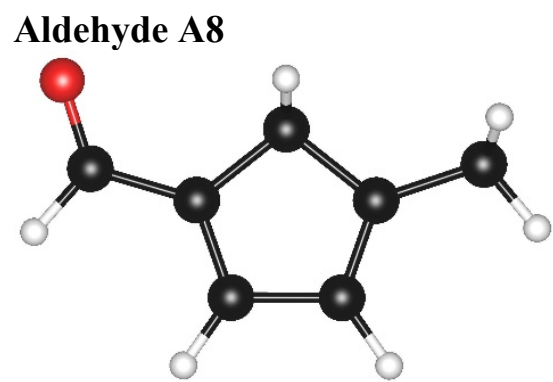

HF $(M 062 X / 6-31+G(d, p))=-346.6127503$ Hartrees

Imaginary Frequencies: none found

Zero-point correction $=0.130759$ (Hartree/Particle)

Thermal correction $=0.098492$ Hartrees

Coordinates from last standard orientation:

\begin{tabular}{|c|c|c|c|}
\hline Center & Atomic & Atomic & Coordinates (Angstroms) \\
\hline Number & Number & Type & $\mathrm{x}$ \\
\hline
\end{tabular}




\begin{tabular}{|c|c|c|c|c|c|}
\hline 1 & 6 & 0 & -0.830027 & 0.295577 & -0.000011 \\
\hline 2 & 6 & 0 & -0.159055 & 1.474461 & -0.000091 \\
\hline 3 & 6 & 0 & 1.275807 & 1.216431 & -0.000142 \\
\hline 4 & 6 & 0 & 1.489414 & -0.120877 & -0.000064 \\
\hline 5 & 1 & 0 & -0.616249 & 2.459337 & -0.000163 \\
\hline 6 & 1 & 0 & 2.045007 & 1.980624 & -0.000259 \\
\hline 7 & 6 & 0 & 2.798092 & -0.841239 & 0.000002 \\
\hline 8 & 1 & 0 & 2.887947 & -1.487244 & -0.880251 \\
\hline 9 & 1 & 0 & 3.635476 & -0.140054 & -0.000817 \\
\hline 10 & 1 & 0 & 2.888565 & -1.485870 & 0.881209 \\
\hline 11 & 6 & 0 & -2.279187 & 0.140251 & -0.000018 \\
\hline 12 & 1 & 0 & -2.856745 & 1.087657 & 0.000009 \\
\hline 13 & 8 & 0 & -2.850382 & -0.933219 & 0.000156 \\
\hline 14 & 6 & 0 & 0.161350 & -0.829900 & 0.000102 \\
\hline 15 & 1 & 0 & 0.040310 & -1.478554 & -0.877663 \\
\hline 16 & 1 & 0 & 0.040379 & -1.478373 & 0.878011 \\
\hline
\end{tabular}

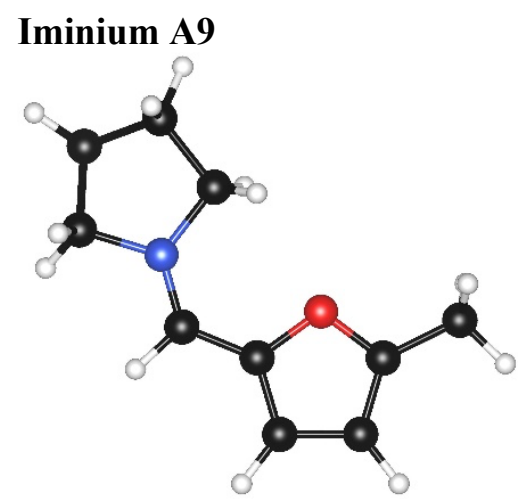

HF $(M 062 X / 6-31+G(d, p))=-519.028205$ Hartrees

Imaginary Frequencies: none found

Zero-point correction $=0.228589$ (Hartree/Particle)

Thermal correction $=0.191270$ Hartrees

\section{Coordinates from last standard orientation:}

\begin{tabular}{|c|c|c|c|c|c|}
\hline \multirow{2}{*}{$\begin{array}{l}\text { Center } \\
\text { Number }\end{array}$} & \multirow{2}{*}{$\begin{array}{l}\text { Atomic } \\
\text { Number }\end{array}$} & \multirow{2}{*}{$\begin{array}{c}\text { Atomic } \\
\text { Type }\end{array}$} & \multicolumn{3}{|c|}{ Coordinates (Angstroms) } \\
\hline & & & $\mathrm{x}$ & $\mathrm{Y}$ & $\mathrm{z}$ \\
\hline 1 & 6 & 0 & 2.071803 & -1.678665 & 0.004074 \\
\hline 2 & 6 & 0 & 3.184241 & -0.819227 & -0.030374 \\
\hline 3 & 6 & 0 & 0.950806 & -0.867405 & 0.026282 \\
\hline 4 & 6 & 0 & -0.408699 & -1.239534 & 0.054838 \\
\hline 5 & 6 & 0 & 2.686100 & 0.466379 & -0.029158 \\
\hline 6 & 6 & 0 & 3.336769 & 1.796960 & -0.058705 \\
\hline 7 & 7 & 0 & -1.452144 & -0.466013 & 0.073777 \\
\hline 8 & 6 & 0 & -1.443953 & 1.019389 & 0.063279 \\
\hline 9 & 6 & 0 & -2.842598 & -0.991811 & 0.079932 \\
\hline 10 & 6 & 0 & -2.919511 & 1.387633 & 0.230088 \\
\hline 11 & 1 & 0 & -0.795916 & 1.386907 & 0.860248 \\
\hline 12 & 6 & 0 & -3.660028 & 0.202663 & -0.399668 \\
\hline 13 & 1 & 0 & -2.903403 & -1.874993 & -0.558052 \\
\hline 14 & 1 & 0 & -3.169167 & 1.470108 & 1.292257 \\
\hline
\end{tabular}




$\begin{array}{rrrrrr}15 & 1 & 0 & -4.702076 & 0.133023 & -0.085526 \\ 16 & 1 & 0 & -1.037387 & 1.348507 & -0.898293 \\ 17 & 1 & 0 & -3.150762 & 2.340271 & -0.247802 \\ 18 & 1 & 0 & -3.631665 & 0.264958 & -1.491982 \\ 19 & 1 & 0 & -0.599647 & -2.311252 & 0.057511 \\ 20 & 1 & 0 & 2.072254 & -2.760433 & 0.012738 \\ 21 & 1 & 0 & 4.228939 & -1.091055 & -0.053606 \\ 22 & 1 & 0 & 3.059039 & 2.378063 & 0.825286 \\ 23 & 1 & 0 & -3.100152 & -1.265023 & 1.108880 \\ 24 & 1 & 0 & 4.419962 & 1.679037 & -0.078654 \\ 25 & 8 & 0 & 1.340123 & 0.443781 & 0.005360 \\ 26 & 1 & 0 & 3.024424 & 2.355435 & -0.945855\end{array}$

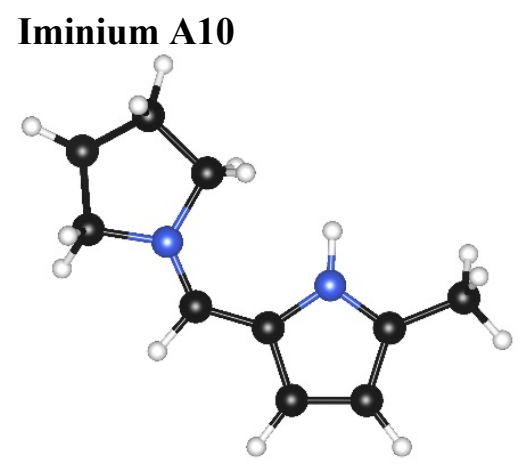

HF $(M 062 X / 6-31+G(d, p))=-499.1821833$ Hartrees

Imaginary Frequencies: none found

Zero-point correction $=0.241203$ (Hartree/Particle)

Thermal correction $=0.202793$ Hartrees

\section{Coordinates from last standard orientation:}

\begin{tabular}{|c|c|c|c|c|c|}
\hline \multirow{2}{*}{$\begin{array}{l}\text { Center } \\
\text { Number }\end{array}$} & \multirow{2}{*}{$\begin{array}{l}\text { Atomic } \\
\text { Number }\end{array}$} & \multirow{2}{*}{$\begin{array}{c}\text { Atomic } \\
\text { Type }\end{array}$} & \multicolumn{3}{|c|}{ Coordinates (Angstroms) } \\
\hline & & & $\mathrm{x}$ & $\mathrm{Y}$ & $\mathrm{Z}$ \\
\hline & & & 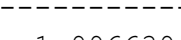 & ----------1 & --------1 \\
\hline 1 & 6 & 0 & 1.996639 & -1.668470 & 0.027647 \\
\hline 2 & 6 & 0 & 3.172804 & -0.930468 & -0.010649 \\
\hline 3 & 6 & 0 & 0.915231 & -0.766556 & 0.026176 \\
\hline 4 & 6 & 0 & -0.432047 & -1.151890 & 0.050023 \\
\hline 5 & 6 & 0 & 2.819682 & 0.425304 & -0.039192 \\
\hline 6 & 6 & 0 & 3.687395 & 1.636511 & -0.068385 \\
\hline 7 & 7 & 0 & -1.516532 & -0.421401 & 0.068411 \\
\hline 8 & 6 & 0 & -1.567167 & 1.053771 & 0.082810 \\
\hline 9 & 6 & 0 & -2.881402 & -1.005074 & 0.064175 \\
\hline 10 & 6 & 0 & -3.058864 & 1.369223 & 0.238010 \\
\hline 11 & 1 & 0 & -0.966175 & 1.434694 & 0.914624 \\
\hline 12 & 6 & 0 & -3.746083 & 0.159803 & -0.405841 \\
\hline 13 & 1 & 0 & -2.905873 & -1.878895 & -0.588889 \\
\hline 14 & 1 & 0 & -3.317224 & 1.432134 & 1.299134 \\
\hline
\end{tabular}




$\begin{array}{rrrrrr}15 & 1 & 0 & -4.785746 & 0.047346 & -0.096183 \\ 16 & 1 & 0 & -1.172792 & 1.427985 & -0.870211 \\ 17 & 1 & 0 & -3.322879 & 2.316372 & -0.233682 \\ 18 & 1 & 0 & -3.716760 & 0.234818 & -1.497326 \\ 19 & 1 & 0 & -0.597018 & -2.227882 & 0.051474 \\ 20 & 1 & 0 & 1.899207 & -2.745760 & 0.055098 \\ 21 & 1 & 0 & 4.185037 & -1.306966 & -0.019788 \\ 22 & 1 & 0 & 3.712983 & 2.117006 & 0.914987 \\ 23 & 1 & 0 & 4.706845 & 1.356759 & -0.334227 \\ 24 & 1 & 0 & 3.328477 & 2.366414 & -0.799114 \\ 25 & 1 & 0 & 1.471294 & 0.509456 & -0.014389 \\ 26 & 7 & 0 & 0.967823 & 1.382965 & -0.048808 \\ 27 & 1 & 0 & & & \end{array}$

\section{Iminium A11}

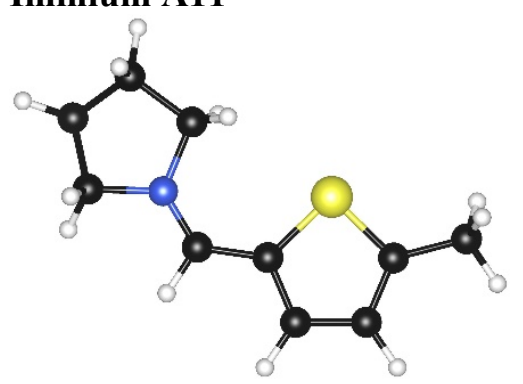

HF $(M 062 X / 6-31+G(d, p))=-841.9915834$ Hartrees

Imaginary Frequencies: none found

Zero-point correction $=0.225272$ (Hartree/Particle)

Thermal correction $=0.186669$ Hartrees

\section{Coordinates from last standard orientation:}

\begin{tabular}{|c|c|c|c|c|c|}
\hline \multirow{2}{*}{$\begin{array}{l}\text { Center } \\
\text { Number }\end{array}$} & \multirow{2}{*}{$\begin{array}{l}\text { Atomic } \\
\text { Number }\end{array}$} & \multirow{2}{*}{$\begin{array}{c}\text { Atomic } \\
\text { Type }\end{array}$} & \multicolumn{3}{|c|}{ Coordinates (Angstroms) } \\
\hline & & & $\mathrm{x}$ & $\mathrm{Y}$ & Z \\
\hline 1 & 6 & 0 & -1.720310 & 1.720016 & 0.020273 \\
\hline 2 & 6 & 0 & -2.995287 & 1.139557 & -0.020358 \\
\hline 3 & 6 & 0 & -0.690694 & 0.782140 & 0.036786 \\
\hline 4 & 6 & 0 & 0.669088 & 1.177416 & 0.061959 \\
\hline 5 & 6 & 0 & -2.956852 & -0.242082 & -0.037188 \\
\hline 6 & 6 & 0 & -4.116709 & -1.185038 & -0.063100 \\
\hline 7 & 7 & 0 & 1.742356 & 0.444838 & 0.079098 \\
\hline 8 & 6 & 0 & 1.791852 & -1.035784 & 0.073044 \\
\hline 9 & 6 & 0 & 3.112215 & 1.022841 & 0.079989 \\
\hline 10 & 6 & 0 & 3.283625 & -1.352559 & 0.214134 \\
\hline 11 & 1 & 0 & 1.184190 & -1.426914 & 0.893287 \\
\hline 12 & 6 & 0 & 3.969756 & -0.135808 & -0.416371 \\
\hline 13 & 1 & 0 & 3.135135 & 1.912600 & -0.550918 \\
\hline 14 & 1 & 0 & 3.550490 & -1.433813 & 1.271986 \\
\hline
\end{tabular}




$\begin{array}{rrrrrr}15 & 1 & 0 & 5.011824 & -0.028657 & -0.112955 \\ 16 & 1 & 0 & 1.382776 & -1.389160 & -0.880286 \\ 17 & 1 & 0 & 3.541294 & -2.292605 & -0.274737 \\ 18 & 1 & 0 & 3.931750 & -0.192626 & -1.508670 \\ 19 & 1 & 0 & 0.841218 & 2.253715 & 0.063738 \\ 20 & 1 & 0 & -1.536596 & 2.789280 & 0.036413 \\ 21 & 1 & 0 & -3.923091 & 1.697979 & -0.038656 \\ 22 & 1 & 0 & -4.217720 & -1.699675 & 0.897187 \\ 23 & 1 & 0 & 3.367293 & 1.296920 & 1.109261 \\ 24 & 1 & 0 & -5.036174 & -0.629560 & -0.253372 \\ 25 & 1 & 0 & -3.999595 & -1.941921 & -0.842529 \\ 26 & 16 & 0 & -1.344337 & -0.835852 & -0.003653\end{array}$

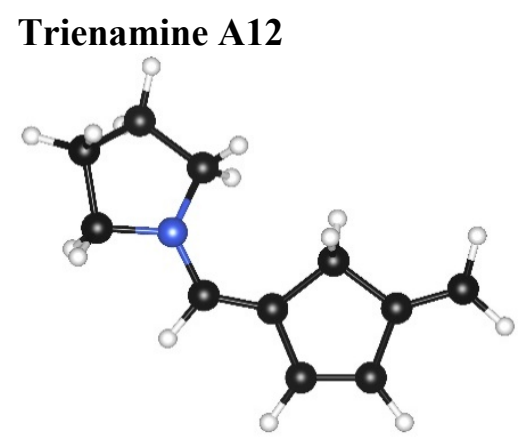

HF $(M 062 X / 6-31+G(d, p))=-482.7037554$ Hartrees

Imaginary Frequencies: none found

Zero-point correction $=0.238156$ (Hartree/Particle)

Thermal correction $=0.200257$ Hartrees

Coordinates from last standard orientation:

\begin{tabular}{|c|c|c|c|c|c|}
\hline \multirow{2}{*}{$\begin{array}{l}\text { Center } \\
\text { Number }\end{array}$} & \multirow{2}{*}{$\begin{array}{l}\text { Atomic } \\
\text { Number }\end{array}$} & \multirow{2}{*}{$\begin{array}{c}\text { Atomic } \\
\text { Type }\end{array}$} & \multicolumn{3}{|c|}{ Coordinates (Angstroms) } \\
\hline & & & $\mathrm{x}$ & $Y$ & Z \\
\hline 1 & 6 & 0 & 2.986169 & 0.525401 & 0.090684 \\
\hline 2 & 6 & 0 & 3.224370 & -0.890424 & -0.193906 \\
\hline 3 & 6 & 0 & 2.053807 & -1.560554 & -0.274431 \\
\hline 4 & 6 & 0 & 0.914644 & -0.680760 & -0.058783 \\
\hline 5 & 1 & 0 & 4.213500 & -1.317128 & -0.313847 \\
\hline 6 & 1 & 0 & 1.949128 & -2.623620 & -0.469634 \\
\hline 7 & 6 & 0 & -0.364344 & -1.113596 & 0.005601 \\
\hline 8 & 1 & 0 & -0.551416 & -2.184647 & -0.079402 \\
\hline 9 & 7 & 0 & -1.496680 & -0.356546 & 0.197985 \\
\hline 10 & 6 & 0 & 3.910472 & 1.483933 & 0.237675 \\
\hline 11 & 1 & 0 & 4.968826 & 1.258491 & 0.150892 \\
\hline 12 & 1 & 0 & 3.634737 & 2.512362 & 0.450269 \\
\hline 13 & 6 & 0 & -2.799636 & -1.009066 & 0.279490 \\
\hline
\end{tabular}




\begin{tabular}{|c|c|c|c|c|c|}
\hline 14 & 1 & 0 & -2.840244 & -1.679950 & 1.143176 \\
\hline 15 & 1 & 0 & -3.011532 & -1.600813 & -0.627711 \\
\hline 16 & 6 & 0 & -3.763206 & 0.168656 & 0.390808 \\
\hline 17 & 1 & 0 & -3.774771 & 0.540132 & 1.420930 \\
\hline 18 & 1 & 0 & -4.784531 & -0.093033 & 0.105329 \\
\hline 19 & 6 & 0 & -3.120024 & 1.201026 & -0.542189 \\
\hline 20 & 1 & 0 & -3.449570 & 2.224287 & -0.348606 \\
\hline 21 & 1 & 0 & -3.364983 & 0.958284 & -1.581625 \\
\hline 22 & 6 & 0 & -1.613387 & 1.015036 & -0.305882 \\
\hline 23 & 1 & 0 & -1.036162 & 1.127396 & -1.231953 \\
\hline 24 & 1 & 0 & -1.225987 & 1.736727 & 0.423880 \\
\hline 25 & 6 & 0 & 1.475178 & 0.711776 & 0.191346 \\
\hline 26 & 1 & 0 & 1.191715 & 1.074725 & 1.186454 \\
\hline 27 & 1 & 0 & 1.133799 & 1.454035 & -0.536530 \\
\hline
\end{tabular}

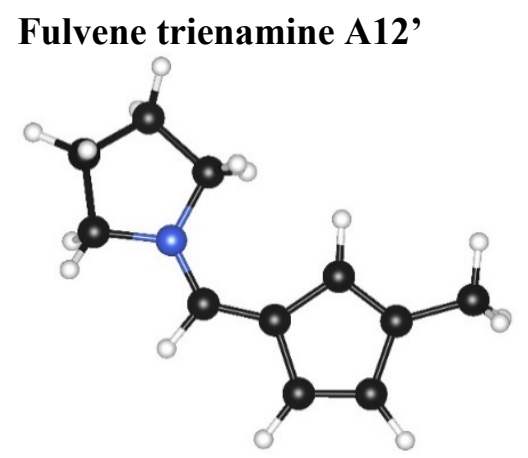

HF $(M 062 X / 6-31+G(d, p))=-482.720686$ Hartrees

Imaginary Frequencies: none found

Zero-point correction $=0.238397$ (Hartree/Particle)

Thermal correction $=0.200207$ Hartrees

Coordinates from last standard orientation:

\begin{tabular}{|c|c|c|c|c|c|}
\hline \multirow{2}{*}{$\begin{array}{l}\text { Center } \\
\text { Number }\end{array}$} & \multirow{2}{*}{$\begin{array}{l}\text { Atomic } \\
\text { Number }\end{array}$} & \multirow{2}{*}{$\begin{array}{c}\text { Atomic } \\
\text { Type }\end{array}$} & \multicolumn{3}{|c|}{ Coordinates (Angstroms) } \\
\hline & & & $\mathrm{x}$ & Y & Z \\
\hline 1 & & & 2868597 & 0454201 & 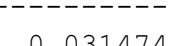 \\
\hline 1 & 6 & 0 & 2.868597 & 0.454201 & 0.031474 \\
\hline 2 & 6 & 0 & 3.160927 & -0.968990 & -0.018590 \\
\hline 3 & 6 & 0 & 1.985378 & -1.658380 & -0.047083 \\
\hline 4 & 6 & 0 & 0.891804 & -0.698183 & -0.022925 \\
\hline 5 & 1 & 0 & 4.158497 & -1.393450 & -0.029803 \\
\hline 6 & 1 & 0 & 1.854234 & -2.733793 & -0.086343 \\
\hline 7 & 6 & 0 & -0.413795 & -1.120447 & -0.038348 \\
\hline 8 & 1 & 0 & -0.585430 & -2.196641 & -0.048984 \\
\hline 9 & 7 & 0 & -1.546153 & -0.399018 & -0.039632 \\
\hline 10 & 6 & 0 & -2.878625 & -1.005029 & -0.034747 \\
\hline 11 & 1 & 0 & -2.911636 & -1.850111 & 0.658871 \\
\hline 12 & 1 & 0 & -3.142797 & -1.367444 & -1.038604 \\
\hline
\end{tabular}




\begin{tabular}{|c|c|c|c|c|c|}
\hline 13 & 6 & 0 & -3.780342 & 0.155887 & 0.382432 \\
\hline 14 & 1 & 0 & -3.772120 & 0.257793 & 1.472808 \\
\hline 15 & 1 & 0 & -4.813559 & 0.022393 & 0.055274 \\
\hline 16 & 6 & 0 & -3.089642 & 1.361604 & -0.266138 \\
\hline 17 & 1 & 0 & -3.379903 & 2.315918 & 0.177781 \\
\hline 18 & 1 & 0 & -3.328913 & 1.396747 & -1.334295 \\
\hline 19 & 6 & 0 & -1.599233 & 1.059706 & -0.076607 \\
\hline 20 & 1 & 0 & -0.978014 & 1.437989 & -0.895308 \\
\hline 21 & 1 & 0 & -1.209761 & 1.470557 & 0.864811 \\
\hline 22 & 6 & 0 & 1.510593 & 0.620644 & 0.027420 \\
\hline 23 & 1 & 0 & 1.006356 & 1.576379 & 0.073724 \\
\hline 24 & 6 & 0 & 3.906864 & 1.533666 & 0.082882 \\
\hline 25 & 1 & 0 & 3.443436 & 2.522744 & 0.135042 \\
\hline 26 & 1 & 0 & 4.556985 & 1.418007 & 0.957441 \\
\hline 27 & 1 & 0 & 4.550523 & 1.507961 & -0.803617 \\
\hline
\end{tabular}

\section{Trieamine A13}

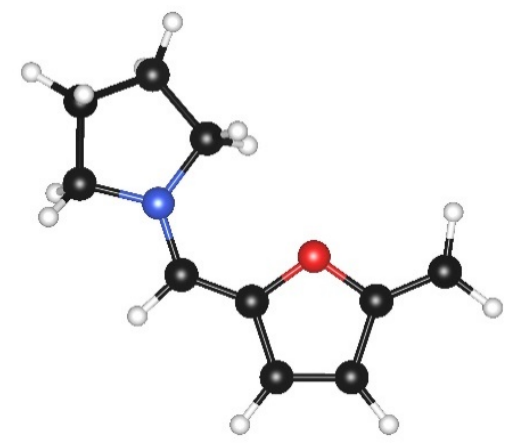

HF $(M 062 X / 6-31+G(d, p))=-518.6058491$ Hartrees

Imaginary Frequencies: none found

Zero-point correction $=0.213928$ (Hartree/Particle)

Thermal correction $=0.175216$ Hartrees

Coordinates from last standard orientation:

\begin{tabular}{cccccc} 
Center & Atomic & Atomic & \multicolumn{3}{c}{ Coordinates (Angstroms) } \\
Number & Number & Type & X & Y & $Z$ \\
-1 & 6 & 0 & 2.745192 & 0.599225 & 0.040828 \\
2 & 6 & 0 & 3.244851 & -0.767393 & -0.035214 \\
3 & 6 & 0 & 2.180404 & -1.597980 & -0.073379 \\
4 & 6 & 0 & 0.984532 & -0.797959 & -0.023546 \\
5 & 1 & 0 & 4.294025 & -1.025899 & -0.054817 \\
6 & 1 & 0 & 2.185420 & -2.678734 & -0.130920 \\
7 & 6 & 0 & -0.308772 & -1.201441 & -0.026831 \\
8 & 1 & 0 & -0.476739 & -2.274060 & -0.063818 \\
9 & 7 & 0 & -1.434911 & -0.439954 & 0.025448 \\
10 & 6 & 0 & 3.391627 & 1.773288 & 0.099996
\end{tabular}




\begin{tabular}{|c|c|c|c|c|c|}
\hline 11 & 1 & 0 & 4.473192 & 1.788434 & 0.094450 \\
\hline 12 & 1 & 0 & 2.848655 & 2.707707 & 0.154995 \\
\hline 13 & 6 & 0 & -2.766022 & -1.033351 & -0.003701 \\
\hline 14 & 1 & 0 & -2.841491 & -1.852471 & 0.719833 \\
\hline 15 & 1 & 0 & -3.003213 & -1.433705 & -1.002591 \\
\hline 16 & 6 & 0 & -3.672495 & 0.147999 & 0.339848 \\
\hline 17 & 1 & 0 & -3.704936 & 0.284655 & 1.426068 \\
\hline 18 & 1 & 0 & -4.693889 & 0.013111 & -0.022768 \\
\hline 19 & 6 & 0 & -2.944664 & 1.325690 & -0.319117 \\
\hline 20 & 1 & 0 & -3.245953 & 2.296659 & 0.079918 \\
\hline 21 & 1 & 0 & -3.142050 & 1.325127 & -1.396667 \\
\hline 22 & 6 & 0 & -1.464806 & 1.021085 & -0.061154 \\
\hline 23 & 1 & 0 & -0.811203 & 1.375387 & -0.863251 \\
\hline 24 & 1 & 0 & -1.109913 & 1.466989 & 0.876919 \\
\hline 25 & 8 & 0 & 1.366674 & 0.533938 & 0.047267 \\
\hline
\end{tabular}

\section{Trienamine A14}

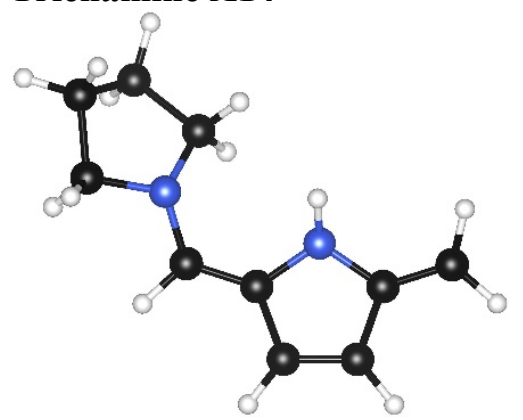

HF $(M 062 X / 6-31+G(d, p))=-498.7478339$ Hartrees Imaginary Frequencies: none found Zero-point correction $=0.226391$ (Hartree/Particle) Thermal correction $=0.188996$ Hartrees

Coordinates from last standard orientation:

\begin{tabular}{|c|c|c|c|c|c|}
\hline \multirow{2}{*}{$\begin{array}{l}\text { Center } \\
\text { Number }\end{array}$} & \multirow{2}{*}{$\begin{array}{l}\text { Atomic } \\
\text { Number }\end{array}$} & \multirow{2}{*}{$\begin{array}{c}\text { Atomic } \\
\text { Type }\end{array}$} & \multicolumn{3}{|c|}{ Coordinates (Angstroms) } \\
\hline & & & $\mathrm{X}$ & $\mathrm{Y}$ & z \\
\hline 1 & 6 & 0 & 2.829793 & 0.569448 & 0.106917 \\
\hline 2 & 6 & 0 & 3.211752 & -0.784762 & -0.307677 \\
\hline 3 & 6 & 0 & 2.107037 & -1.555518 & -0.376476 \\
\hline 4 & 6 & 0 & 0.945933 & -0.751857 & -0.027353 \\
\hline 5 & 7 & 0 & 1.431912 & 0.547781 & 0.184725 \\
\hline 6 & 1 & 0 & 0.933279 & 1.196431 & 0.774274 \\
\hline 7 & 1 & 0 & 4.233420 & -1.077107 & -0.509965 \\
\hline 8 & 1 & 0 & 2.050578 & -2.600391 & -0.653932 \\
\hline 9 & 6 & 0 & -0.344221 & -1.147847 & 0.055164 \\
\hline 10 & 1 & 0 & -0.573822 & -2.205030 & -0.063971 \\
\hline 11 & 7 & 0 & -1.413410 & -0.299447 & 0.310760 \\
\hline
\end{tabular}




$\begin{array}{rrrrrr}12 & 6 & 0 & 3.632216 & 1.620059 & 0.364573 \\ 13 & 1 & 0 & 4.705196 & 1.513046 & 0.273203 \\ 14 & 1 & 0 & 3.236071 & 2.582317 & 0.667397 \\ 15 & 6 & 0 & -2.721337 & -0.909461 & 0.543053 \\ 16 & 1 & 0 & -2.767170 & -1.361328 & 1.538982 \\ 17 & 1 & 0 & -2.924096 & -1.699689 & -0.201002 \\ 18 & 6 & 0 & -3.695431 & 0.252046 & 0.366099 \\ 19 & 1 & 0 & -3.725768 & 0.856623 & 1.279097 \\ 20 & 1 & 0 & -4.710458 & -0.081866 & 0.139434 \\ 21 & 6 & 0 & -3.045561 & 1.042091 & -0.774043 \\ 22 & 1 & 0 & -3.403518 & 2.071489 & -0.850872 \\ 23 & 1 & 0 & -3.242905 & 0.540431 & -1.727833 \\ 24 & 6 & 0 & -1.550400 & 0.960462 & -0.444271 \\ 25 & 1 & 0 & -0.916551 & 0.939488 & -1.336890 \\ 26 & 1 & 0 & -1.242464 & 1.819290 & 0.167771 \\ -------------------------------1\end{array}$

\section{Fulvene trienamine A14'}

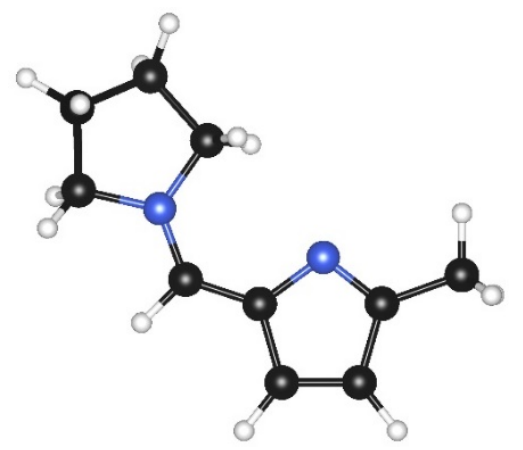

HF $(M 062 X / 6-31+G(d, p))=-498.7768887$ Hartrees

Imaginary Frequencies: none found

Zero-point correction $=0.227128$ (Hartree/Particle)

Thermal correction $=0.189429$ Hartrees

Coordinates from last standard orientation:

\begin{tabular}{|c|c|c|c|c|c|}
\hline \multirow{2}{*}{$\begin{array}{l}\text { Center } \\
\text { Number }\end{array}$} & \multirow{2}{*}{$\begin{array}{l}\text { Atomic } \\
\text { Number }\end{array}$} & \multirow{2}{*}{$\begin{array}{c}\text { Atomic } \\
\text { Type }\end{array}$} & \multicolumn{3}{|c|}{ Coordinates (Angstroms) } \\
\hline & & & $\mathrm{x}$ & Y & Z \\
\hline 1 & 6 & 0 & 2.692671 & 0.481895 & 0.026678 \\
\hline 2 & 6 & 0 & 3.178172 & -0.880804 & 0.015161 \\
\hline 3 & 6 & 0 & 2.066997 & -1.677579 & -0.016139 \\
\hline 4 & 6 & 0 & 0.942446 & -0.777379 & -0.024065 \\
\hline 5 & 1 & 0 & 4.216558 & -1.187257 & 0.029303 \\
\hline 6 & 1 & 0 & 2.017508 & -2.759682 & -0.033153 \\
\hline 7 & 6 & 0 & -0.374391 & -1.184693 & -0.050228 \\
\hline 8 & 1 & 0 & -0.562355 & -2.257509 & -0.061540 \\
\hline 9 & 7 & 0 & -1.474110 & -0.431614 & -0.059064 \\
\hline 10 & 6 & 0 & -2.825811 & -0.998428 & -0.068463 \\
\hline
\end{tabular}




$\begin{array}{rrrrrr}11 & 1 & 0 & -2.882023 & -1.861841 & 0.600558 \\ 12 & 1 & 0 & -3.095563 & -1.323777 & -1.082837 \\ 13 & 6 & 0 & -3.695179 & 0.176488 & 0.376632 \\ 14 & 1 & 0 & -3.685494 & 0.251199 & 1.469281 \\ 15 & 1 & 0 & -4.731096 & 0.077308 & 0.045774 \\ 16 & 6 & 0 & -2.968279 & 1.376841 & -0.241176 \\ 17 & 1 & 0 & -3.237668 & 2.327830 & 0.222601 \\ 18 & 1 & 0 & -3.200938 & 1.442503 & -1.309756 \\ 19 & 6 & 0 & -1.488001 & 1.037726 & -0.046131 \\ 20 & 1 & 0 & -0.827573 & 1.425675 & -0.823678 \\ 21 & 1 & 0 & -1.093341 & 1.394171 & 0.912262 \\ 22 & 6 & 0 & 3.546325 & 1.711766 & 0.062109 \\ 23 & 1 & 0 & 4.179548 & 1.725047 & 0.955578 \\ 24 & 1 & 0 & 2.913381 & 2.600901 & 0.066281 \\ 25 & 1 & 0 & 1.378396 & 0.545287 & 0.003590 \\ 26 & 7 & 0 & 0 & & \end{array}$

\section{Trienamine A15}

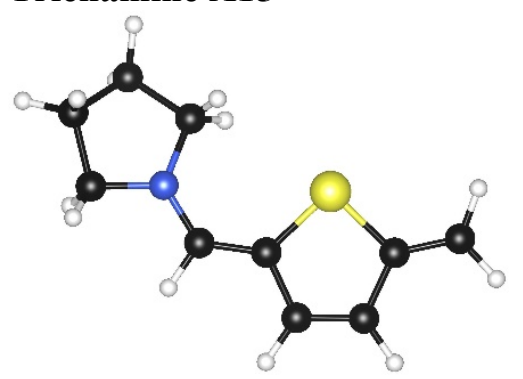

HF $(M 062 X / 6-31+G(d, p))=-841.5716629$ Hartrees

Imaginary Frequencies: none found

Zero-point correction $=0.210916$ (Hartree/Particle)

Thermal correction $=0.171406$ Hartrees

\section{Coordinates from last standard orientation:}

\begin{tabular}{rrrrrr} 
Center & $\begin{array}{c}\text { Atomic } \\
\text { Number }\end{array}$ & $\begin{array}{c}\text { Atomic } \\
\text { Number }\end{array}$ & \multicolumn{3}{c}{ Coordinates } \\
Type & X Angstroms) & Y & $Z$ \\
1 & 6 & 0 & -3.055180 & -0.346298 & 0.026575 \\
2 & 6 & 0 & -3.062135 & 1.102553 & -0.150453 \\
3 & 6 & 0 & -1.838690 & 1.667574 & -0.180820 \\
4 & 6 & 0 & -0.728078 & 0.749543 & -0.036290 \\
5 & 1 & 0 & -3.997682 & 1.641832 & -0.245034 \\
6 & 1 & 0 & -1.660578 & 2.731652 & -0.303701 \\
7 & 6 & 0 & 0.567522 & 1.153284 & -0.002579 \\
8 & 1 & 0 & 0.740098 & 2.227447 & -0.059326 \\
9 & 7 & 0 & 1.704859 & 0.414634 & 0.112913 \\
10 & 6 & 0 & -4.119293 & -1.163239 & 0.090603 \\
11 & 1 & 0 & -5.118806 & -0.750368 & 0.007663 \\
12 & 1 & 0 & -4.020571 & -2.234036 & 0.224608 \\
13 & 6 & 0 & 3.016371 & 1.056607 & 0.154104
\end{tabular}




$\begin{array}{rrrrrr}14 & 1 & 0 & 3.068547 & 1.778395 & 0.975568 \\ 15 & 1 & 0 & 3.225683 & 1.588679 & -0.787915 \\ 16 & 6 & 0 & 3.969654 & -0.122597 & 0.336902 \\ 17 & 1 & 0 & 4.014637 & -0.401804 & 1.394943 \\ 18 & 1 & 0 & 4.982564 & 0.100327 & -0.005192 \\ 19 & 6 & 0 & 3.284907 & -1.228782 & -0.473454 \\ 20 & 1 & 0 & 3.625552 & -2.232471 & -0.210712 \\ 21 & 1 & 0 & 3.471005 & -1.073842 & -1.541617 \\ 22 & 6 & 0 & 1.796487 & -1.019756 & -0.170152 \\ 23 & 1 & 0 & 1.156534 & -1.283277 & -1.018283 \\ 24 & 1 & 0 & 1.476445 & -1.610291 & 0.697772 \\ 25 & 16 & 0 & -1.367927 & -0.904876 & 0.157139\end{array}$

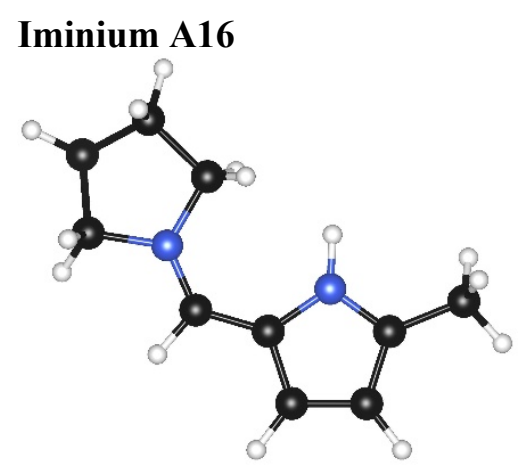

HF $(M 062 X / 6-31+G(d, p))=-483.1133476$ Hartrees

Imaginary Frequencies: none found Zero-point correction $=0.251329$ (Hartree/Particle)

Thermal correction $=0.213321$ Hartrees

Coordinates from last standard orientation:

\begin{tabular}{|c|c|c|c|c|c|}
\hline \multirow{2}{*}{$\begin{array}{l}\text { Center } \\
\text { Number }\end{array}$} & \multirow{2}{*}{$\begin{array}{l}\text { Atomic } \\
\text { Number }\end{array}$} & \multirow{2}{*}{$\begin{array}{c}\text { Atomic } \\
\text { Type }\end{array}$} & \multicolumn{3}{|c|}{ Coordinates (Angstroms) } \\
\hline & & & $\mathrm{x}$ & $\mathrm{Y}$ & Z \\
\hline 1 & 6 & 0 & -1.886537 & -1.632074 & -0.005783 \\
\hline 2 & 6 & 0 & -3.159509 & -0.975359 & 0.040865 \\
\hline 3 & 6 & 0 & -0.865688 & -0.707269 & -0.044241 \\
\hline 4 & 6 & 0 & 0.482677 & -1.130099 & -0.070862 \\
\hline 5 & 6 & 0 & -2.963655 & 0.373338 & 0.031017 \\
\hline 6 & 6 & 0 & -3.997190 & 1.443894 & 0.064806 \\
\hline 7 & 7 & 0 & 1.567462 & -0.407366 & -0.075299 \\
\hline 8 & 6 & 0 & 1.632738 & 1.071582 & -0.035825 \\
\hline 9 & 6 & 0 & 2.929699 & -0.999671 & -0.086535 \\
\hline 10 & 6 & 0 & 3.122754 & 1.374434 & -0.211741 \\
\hline 11 & 1 & 0 & 1.006168 & 1.492960 & -0.824010 \\
\hline 12 & 6 & 0 & 3.807807 & 0.148021 & 0.400483 \\
\hline
\end{tabular}




\begin{tabular}{|c|c|c|c|c|c|}
\hline 13 & 1 & 0 & 2.949204 & -1.888255 & 0.546478 \\
\hline 14 & 1 & 0 & 3.366094 & 1.453901 & -1.275577 \\
\hline 15 & 1 & 0 & 4.843713 & 0.032365 & 0.079673 \\
\hline 16 & 1 & 0 & 1.261928 & 1.406915 & 0.938833 \\
\hline 17 & 1 & 0 & 3.403342 & 2.310643 & 0.272180 \\
\hline 18 & 1 & 0 & 3.788641 & 0.200822 & 1.493555 \\
\hline 19 & 1 & 0 & 0.651129 & -2.206974 & -0.082626 \\
\hline 20 & 1 & 0 & -1.745815 & -2.709167 & -0.007318 \\
\hline 21 & 1 & 0 & -4.118064 & -1.477421 & 0.079215 \\
\hline 22 & 1 & 0 & -3.942072 & 2.060843 & -0.838755 \\
\hline 23 & 1 & 0 & 3.175415 & -1.280860 & -1.116285 \\
\hline 24 & 1 & 0 & -5.000507 & 1.022370 & 0.136176 \\
\hline 25 & 1 & 0 & -3.835386 & 2.110297 & 0.918710 \\
\hline 26 & 6 & 0 & -1.491390 & 0.666817 & -0.030379 \\
\hline 27 & 1 & 0 & -1.264441 & 1.247653 & -0.933811 \\
\hline 28 & 1 & 0 & -1.181829 & 1.273787 & 0.829821 \\
\hline
\end{tabular}

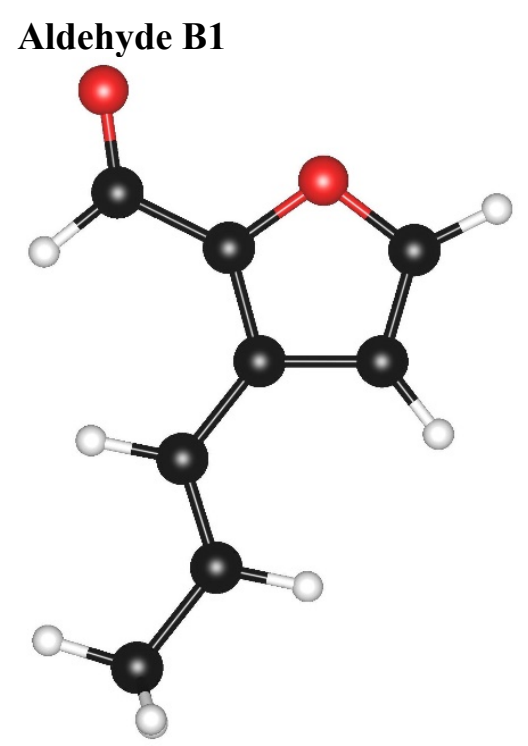

HF $(M 062 X / 6-31+G(d, p))=-459.8920817$ Hartrees

Imaginary Frequencies: none found

Zero-point correction $=0.142254$ (Hartree/Particle)

Thermal correction $=0.107009$ Hartrees

Coordinates from last standard orientation:

\begin{tabular}{|c|c|c|c|c|c|}
\hline \multirow{2}{*}{$\begin{array}{l}\text { Center } \\
\text { Number }\end{array}$} & \multirow{2}{*}{$\begin{array}{l}\text { Atomic } \\
\text { Number }\end{array}$} & \multirow{2}{*}{$\begin{array}{c}\text { Atomic } \\
\text { Type }\end{array}$} & \multicolumn{3}{|c|}{ Coordinates (Angstroms) } \\
\hline & & & $x$ & Y & Z \\
\hline & & & & -------- & --------- \\
\hline 1 & 6 & 0 & 0.156592 & 0.332642 & 0.000118 \\
\hline 2 & 6 & 0 & -1.124296 & -0.179309 & -0.000104 \\
\hline 3 & 6 & 0 & -1.618166 & -1.550818 & -0.000173 \\
\hline 4 & 1 & 0 & -0.825187 & -2.322712 & -0.000582 \\
\hline 5 & 6 & 0 & 1.401428 & -0.432246 & 0.000331 \\
\hline
\end{tabular}




$\begin{array}{rrrrrr}6 & 6 & 0 & 2.623805 & 0.112161 & -0.000054 \\ 7 & 6 & 0 & 3.899091 & -0.671982 & -0.000090 \\ 8 & 1 & 0 & 4.505492 & -0.429055 & 0.878932 \\ 9 & 1 & 0 & 3.703921 & -1.747239 & 0.001843 \\ 10 & 1 & 0 & 1.312386 & -1.517116 & 0.000871 \\ 11 & 1 & 0 & 2.720925 & 1.197655 & -0.000481 \\ 12 & 6 & 0 & -1.349960 & 1.981493 & 0.000067 \\ 13 & 1 & 0 & -1.942345 & 2.883819 & 0.000063 \\ 14 & 6 & 0 & -0.008109 & 1.757558 & 0.000055 \\ 15 & 1 & 0 & 0.763585 & 2.512637 & 0.000161 \\ 16 & 1 & 0 & 4.503512 & -0.431944 & -0.881302 \\ 17 & 8 & 0 & -2.036948 & 0.826516 & -0.000184 \\ 18 & 8 & 0 & -2.791127 & -1.856896 & 0.000133 \\ --------------------------------------------------1\end{array}$

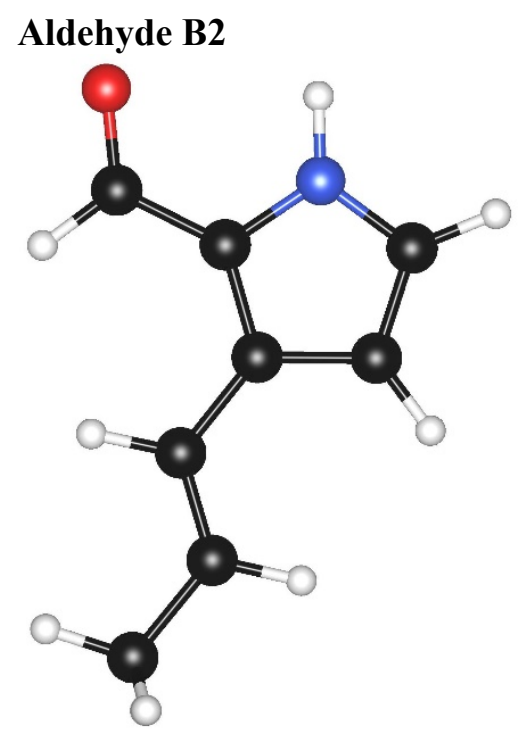

HF $($ M062X/6-31+G(d,p)) = -440.0542266 Hartrees

Imaginary Frequencies: none found

Zero-point correction $=0.155004$ (Hartree/Particle)

Thermal correction $=0.119651$ Hartrees

Coordinates from last standard orientation:

\begin{tabular}{cccccc} 
Center & Atomic & Atomic & \multicolumn{3}{c}{ Coordinates } \\
Number & Number & Type & $X$ & Y Angtroms) \\
-1 & 6 & 0 & 0.170557 & 0.344118 & 0.000294 \\
2 & 6 & 0 & -1.113486 & -0.207302 & 0.000152 \\
3 & 6 & 0 & -1.593894 & -1.574674 & 0.000029 \\
4 & 1 & 0 & -0.825434 & -2.368153 & 0.000292 \\
5 & 6 & 0 & 1.420573 & -0.414254 & 0.000492
\end{tabular}




$\begin{array}{rrrrrr}6 & 6 & 0 & 2.645751 & 0.123997 & -0.000261 \\ 7 & 6 & 0 & 3.917927 & -0.666789 & -0.000212 \\ 8 & 1 & 0 & 4.526751 & -0.429396 & 0.878934 \\ 9 & 1 & 0 & 3.716092 & -1.741149 & 0.001731 \\ 10 & 1 & 0 & 1.332808 & -1.500150 & 0.001360 \\ 11 & 1 & 0 & 2.747641 & 1.209094 & -0.001064 \\ 12 & 6 & 0 & -1.360946 & 2.008493 & -0.000144 \\ 13 & 1 & 0 & -1.894774 & 2.947372 & -0.000345 \\ 14 & 6 & 0 & -0.002751 & 1.754788 & 0.000230 \\ 15 & 1 & 0 & 0.773333 & 2.506068 & 0.000388 \\ 16 & 1 & 0 & 4.524921 & -0.432292 & -0.881427 \\ 17 & 8 & 0 & -2.781778 & -1.853912 & -0.000277 \\ 18 & 7 & 0 & -2.018887 & 0.823082 & -0.000165 \\ 19 & 1 & 0 & -3.017293 & 0.668069 & 0.000032 \\ -----------------------------------------------1\end{array}$

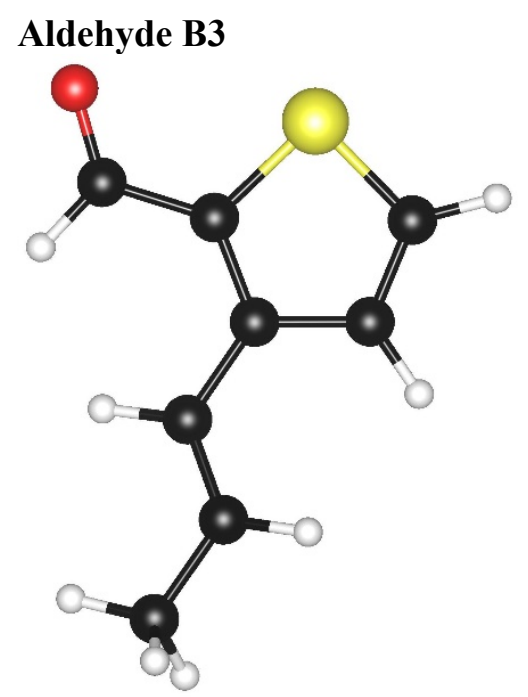

HF $(M 062 X / 6-31+G(d, p))=-782.8629443$ Hartrees

Imaginary Frequencies: none found

Zero-point correction $=0.138668$ (Hartree/Particle)

Thermal correction $=0.102485$ Hartrees

Coordinates from last standard orientation:

\begin{tabular}{|c|c|c|c|c|c|}
\hline \multirow{2}{*}{$\begin{array}{l}\text { Center } \\
\text { Number }\end{array}$} & \multirow{2}{*}{$\begin{array}{l}\text { Atomic } \\
\text { Number }\end{array}$} & \multirow{2}{*}{$\begin{array}{c}\text { Atomic } \\
\text { Type }\end{array}$} & \multicolumn{3}{|c|}{ Coordinates (Angstroms) } \\
\hline & & & $x$ & Y & Z \\
\hline--- & & & ( & ( & ------ \\
\hline 1 & 6 & 0 & -0.346814 & -0.274220 & -0.068014 \\
\hline 2 & 6 & 0 & 0.863826 & 0.401062 & -0.025519 \\
\hline 3 & 6 & 0 & 1.101979 & 1.842626 & -0.000699 \\
\hline 4 & 1 & 0 & 0.199003 & 2.479863 & -0.021404 \\
\hline 5 & 6 & 0 & -1.659162 & 0.379713 & -0.125736 \\
\hline 6 & 6 & 0 & -2.827082 & -0.232556 & 0.104243 \\
\hline
\end{tabular}




$\begin{array}{rrrrrr}7 & 6 & 0 & -4.161055 & 0.443817 & 0.035167 \\ 8 & 1 & 0 & -4.683485 & 0.373690 & 0.995173 \\ 9 & 1 & 0 & -4.057680 & 1.500216 & -0.225293 \\ 10 & 1 & 0 & -1.674469 & 1.439573 & -0.371163 \\ 11 & 1 & 0 & -2.833113 & -1.289184 & 0.370055 \\ 12 & 6 & 0 & 1.176432 & -2.033636 & -0.001317 \\ 13 & 1 & 0 & 1.591211 & -3.032799 & 0.008566 \\ 14 & 6 & 0 & -0.144064 & -1.690428 & -0.056519 \\ 15 & 1 & 0 & -0.943562 & -2.418934 & -0.109864 \\ 16 & 1 & 0 & -4.802421 & -0.034604 & -0.712885 \\ 17 & 8 & 0 & 2.207938 & 2.342223 & 0.044619 \\ 18 & 16 & 0 & 2.219791 & -0.673368 & 0.033764 \\ -----------------------------------------------------\end{array}$

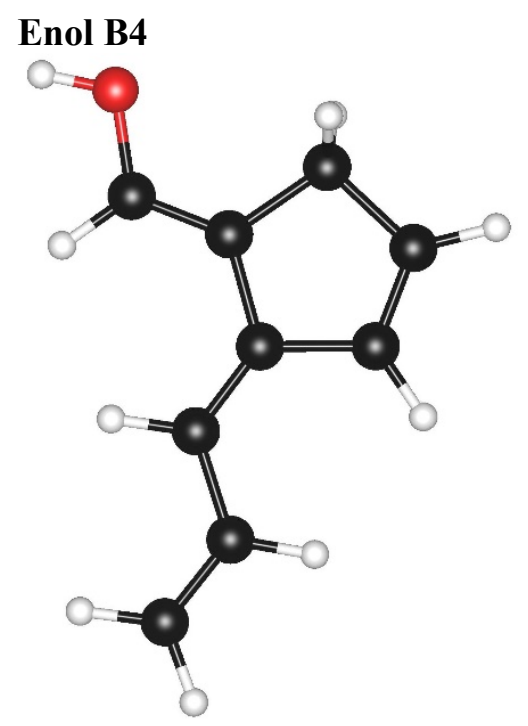

HF $(M 062 X / 6-31+G(d, p))=-423.962005$ Hartrees

Imaginary Frequencies: none found

Zero-point correction $=0.165626$ (Hartree/Particle)

Thermal correction $=0.130196$ Hartrees

Coordinates from last standard orientation:

\begin{tabular}{|c|c|c|c|c|c|}
\hline \multirow{2}{*}{$\begin{array}{l}\text { Center } \\
\text { Number }\end{array}$} & \multirow{2}{*}{$\begin{array}{l}\text { Atomic } \\
\text { Number }\end{array}$} & \multirow{2}{*}{$\begin{array}{c}\text { Atomic } \\
\text { Type }\end{array}$} & \multicolumn{3}{|c|}{ Coordinates (Angstroms) } \\
\hline & & & $x$ & Y & Z \\
\hline--- & & & $---2--1$ & $\cdots--$ & ---------- \\
\hline 1 & 6 & 0 & -0.338758 & 0.264310 & 0.000026 \\
\hline 2 & 6 & 0 & 1.076592 & -0.165825 & -0.000039 \\
\hline 3 & 6 & 0 & 1.534391 & -1.419239 & 0.000128 \\
\hline 4 & 1 & 0 & 0.882328 & -2.289208 & 0.000318 \\
\hline 5 & 6 & 0 & -1.438569 & -0.523758 & -0.000098 \\
\hline
\end{tabular}




\begin{tabular}{|c|c|c|c|c|c|}
\hline 6 & 6 & 0 & -2.810125 & -0.048347 & 0.000043 \\
\hline 7 & 6 & 0 & -3.881241 & -0.855807 & -0.000064 \\
\hline 8 & 1 & 0 & -4.890588 & -0.460108 & 0.000061 \\
\hline 9 & 1 & 0 & -3.770003 & -1.937185 & -0.000278 \\
\hline 10 & 1 & 0 & -1.312150 & -1.606048 & -0.000320 \\
\hline 11 & 1 & 0 & -2.967748 & 1.028445 & 0.000260 \\
\hline 12 & 6 & 0 & 0.965582 & 2.185159 & 0.000026 \\
\hline 13 & 1 & 0 & 1.254164 & 3.230646 & 0.000034 \\
\hline 14 & 6 & 0 & -0.298314 & 1.735944 & 0.000162 \\
\hline 15 & 1 & 0 & -1.180390 & 2.364792 & 0.000314 \\
\hline 16 & 6 & 0 & 1.967252 & 1.059445 & -0.000187 \\
\hline 17 & 1 & 0 & 2.623661 & 1.087570 & -0.879163 \\
\hline 18 & 1 & 0 & 2.624001 & 1.087530 & 0.878536 \\
\hline 19 & 8 & 0 & 2.878252 & -1.661293 & -0.000064 \\
\hline 20 & 1 & 0 & 3.049848 & -2.607385 & 0.000770 \\
\hline
\end{tabular}

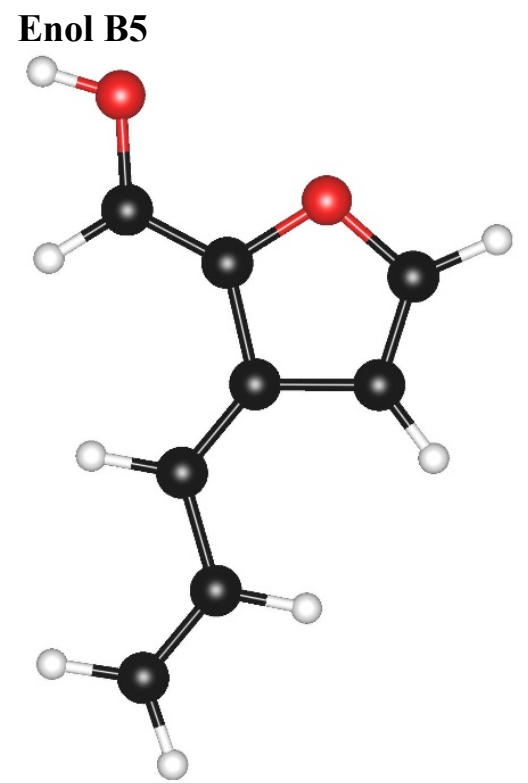

HF $(M 062 X / 6-31+G(d, p))=-459.8540095$ Hartrees

Imaginary Frequencies: none found

Zero-point correction $=0.141517$ (Hartree/Particle)

Thermal correction $=0.106321$ Hartrees

Coordinates from last standard orientation:

\begin{tabular}{cccccc} 
Center & Atomic & Atomic & \multicolumn{3}{c}{ Coordinates (Angstroms) } \\
Number & Number & Type & $X$ & $Y$ & $Z$ \\
-1 & 6 & 0 & -0.350825 & 0.264652 & 0.000063 \\
2 & 6 & 0 & 1.063419 & -0.144250 & 0.000032 \\
3 & 6 & 0 & 1.621940 & -1.360345 & 0.000087
\end{tabular}




\begin{tabular}{|c|c|c|c|c|c|}
\hline 4 & 1 & 0 & 0.999224 & -2.248373 & 0.000162 \\
\hline 5 & 6 & 0 & -1.442305 & -0.538923 & 0.000035 \\
\hline 6 & 6 & 0 & -2.809674 & -0.060985 & -0.000030 \\
\hline 7 & 6 & 0 & -3.887598 & -0.860485 & -0.000070 \\
\hline 8 & 1 & 0 & -4.893381 & -0.456151 & -0.000167 \\
\hline 9 & 1 & 0 & -3.785325 & -1.942601 & -0.000017 \\
\hline 10 & 1 & 0 & -1.305317 & -1.619116 & 0.000057 \\
\hline 11 & 1 & 0 & -2.958075 & 1.017615 & -0.000095 \\
\hline 12 & 8 & 0 & 1.865207 & 0.986859 & -0.000010 \\
\hline 13 & 6 & 0 & 1.023095 & 2.057127 & -0.000093 \\
\hline 14 & 1 & 0 & 1.513580 & 3.020366 & -0.000086 \\
\hline 15 & 6 & 0 & -0.277143 & 1.725747 & 0.000126 \\
\hline 16 & 1 & 0 & -1.105074 & 2.417643 & 0.000304 \\
\hline 17 & 8 & 0 & 2.971270 & -1.516077 & -0.000282 \\
\hline 18 & 1 & 0 & 3.197087 & -2.450868 & 0.001281 \\
\hline
\end{tabular}

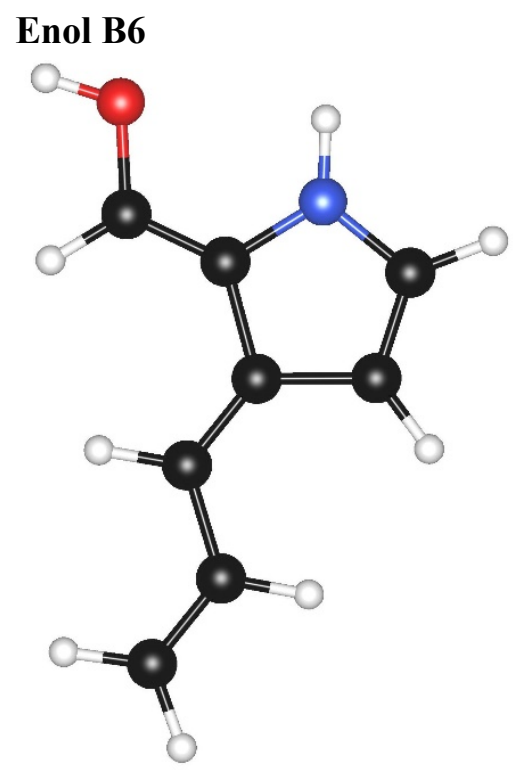

HF $(M 062 X / 6-31+G(d, p))=-440.0033034$ Hartrees

Imaginary Frequencies: none found Zero-point correction $=0.152735$ (Hartree/Particle)

Thermal correction $=0.116901$ Hartrees

Coordinates from last standard orientation:

\begin{tabular}{cccccc} 
Center & Atomic & Atomic & \multicolumn{3}{c}{ Coordinates } \\
Number & Number & Type & X & Y & $Z$ \\
-1 & 6 & 0 & -0.356701 & 0.274819 & 0.000033 \\
2 & 6 & 0 & 1.056893 & -0.169372 & 0.000443 \\
3 & 6 & 0 & 1.584701 & -1.402207 & -0.000013
\end{tabular}




$\begin{array}{rrrrrr}4 & 1 & 0 & 0.982242 & -2.302049 & -0.000626 \\ 5 & 6 & 0 & -1.449809 & -0.531306 & 0.000238 \\ 6 & 6 & 0 & -2.816112 & -0.055424 & -0.000043 \\ 7 & 6 & 0 & -3.896480 & -0.853342 & -0.000101 \\ 8 & 1 & 0 & -4.901644 & -0.447312 & -0.000387 \\ 9 & 1 & 0 & -3.796151 & -1.935738 & 0.000098 \\ 10 & 1 & 0 & -1.311820 & -1.611526 & 0.000572 \\ 11 & 1 & 0 & -2.963693 & 1.023366 & -0.000291 \\ 12 & 6 & 0 & 1.018638 & 2.093959 & -0.000152 \\ 13 & 1 & 0 & 1.447794 & 3.087094 & -0.000358 \\ 14 & 6 & 0 & -0.287204 & 1.735112 & -0.000254 \\ 15 & 1 & 0 & -1.124588 & 2.415147 & -0.000395 \\ 16 & 8 & 0 & 2.951331 & -1.529565 & -0.001222 \\ 17 & 1 & 0 & 3.203512 & -2.457111 & 0.005278 \\ 18 & 7 & 0 & 1.840591 & 0.989771 & 0.000898 \\ 19 & 1 & 0 & 2.846008 & 0.982824 & -0.001299 \\ --------------------------------------------1\end{array}$

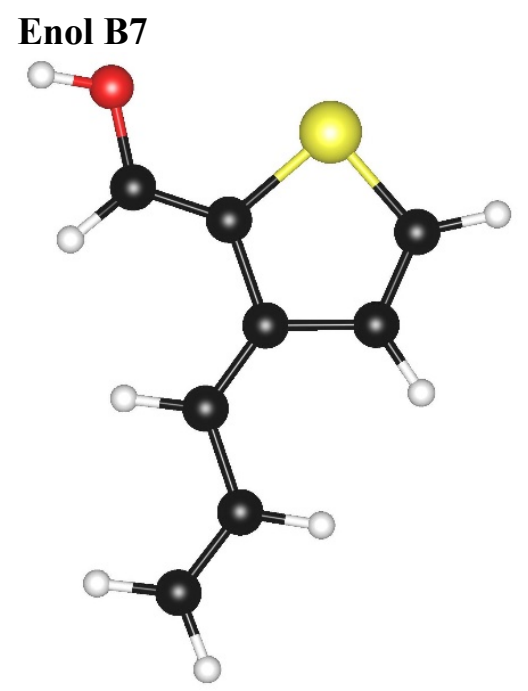

HF $(M 062 X / 6-31+G(d, p))=-782.8244225$ Hartrees

Imaginary Frequencies: none found

Zero-point correction $=0.138690$ (Hartree/Particle)

Thermal correction $=0.102729$ Hartrees

Coordinates from last standard orientation:

\begin{tabular}{cccccr} 
Center & Atomic & Atomic & \multicolumn{3}{c}{ Coordinates } \\
Number & Number & Type & X Angstroms) & Y & $Z$ \\
-1 & 6 & 0 & 0.530561 & -0.211753 & -0.032191 \\
2 & 6 & 0 & -0.822248 & 0.384908 & 0.001438 \\
3 & 6 & 0 & -1.142555 & 1.682648 & -0.077888 \\
4 & 1 & 0 & -0.389028 & 2.456814 & -0.190392
\end{tabular}




\begin{tabular}{|c|c|c|c|c|c|}
\hline 5 & 6 & 0 & 1.689649 & 0.491100 & 0.040353 \\
\hline 6 & 6 & 0 & 3.021498 & -0.078152 & -0.020263 \\
\hline 7 & 6 & 0 & 4.145852 & 0.649812 & 0.062597 \\
\hline 8 & 1 & 0 & 5.125339 & 0.187824 & 0.012848 \\
\hline 9 & 1 & 0 & 4.109784 & 1.729618 & 0.182174 \\
\hline 10 & 1 & 0 & 1.643536 & 1.572363 & 0.160605 \\
\hline 11 & 1 & 0 & 3.106360 & -1.156189 & -0.140268 \\
\hline 12 & 6 & 0 & -0.862469 & -2.109015 & -0.042083 \\
\hline 13 & 1 & 0 & -1.179739 & -3.144231 & -0.054998 \\
\hline 14 & 6 & 0 & 0.404393 & -1.666396 & -0.108656 \\
\hline 15 & 1 & 0 & 1.253870 & -2.333442 & -0.179637 \\
\hline 16 & 8 & 0 & -2.442206 & 2.074794 & -0.011325 \\
\hline 17 & 1 & 0 & -2.525648 & 3.015794 & -0.192310 \\
\hline 18 & 16 & 0 & -2.087182 & -0.861614 & 0.097046 \\
\hline
\end{tabular}

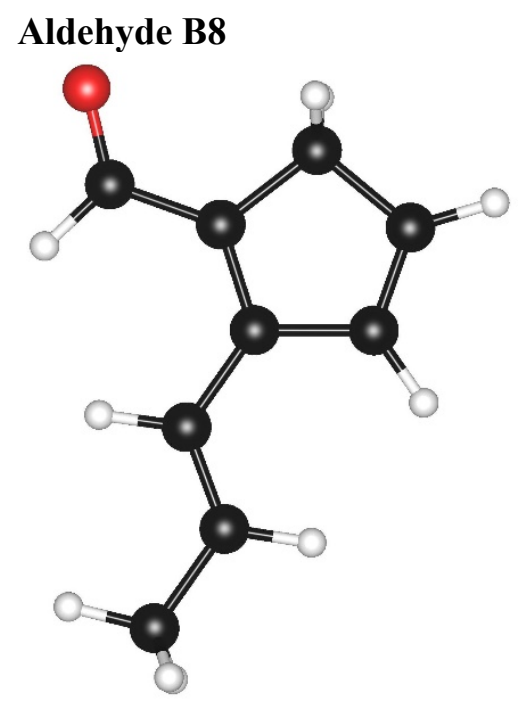

HF $(M 062 X / 6-31+G(d, p))=-423.9821478$ Hartrees

Imaginary Frequencies: none found

Zero-point correction $=0.165110$ (Hartree/Particle)

Thermal correction $=0.129708$ Hartrees

Coordinates from last standard orientation:

\begin{tabular}{cccccc} 
Center & Atomic & Atomic & \multicolumn{3}{c}{ Coordinates } \\
Number & Number & Type & $\mathrm{X}$ & $\mathrm{Y}$ & $\mathrm{Z}$ \\
-1 & 6 & 0 & -0.133036 & 0.304327 & -0.000013 \\
2 & 6 & 0 & 1.134397 & -0.213557 & -0.000130 \\
3 & 6 & 0 & 1.520053 & -1.617991 & -0.000032 \\
4 & 1 & 0 & 0.699936 & -2.361707 & -0.000108
\end{tabular}




$\begin{array}{rrrrrr}5 & 6 & 0 & -1.388875 & -0.440435 & 0.000014 \\ 6 & 6 & 0 & -2.604974 & 0.123106 & 0.000029 \\ 7 & 6 & 0 & -3.890813 & -0.642730 & -0.000027 \\ 8 & 1 & 0 & -4.492409 & -0.391292 & -0.880104 \\ 9 & 1 & 0 & -3.712179 & -1.720735 & 0.000317 \\ 10 & 1 & 0 & -1.322556 & -1.526616 & 0.000017 \\ 11 & 1 & 0 & -2.690278 & 1.208923 & 0.000057 \\ 12 & 6 & 0 & 1.276827 & 2.122981 & 0.000026 \\ 13 & 1 & 0 & 1.662900 & 3.135474 & 0.000025 \\ 14 & 6 & 0 & -0.022682 & 1.772252 & 0.000106 \\ 15 & 1 & 0 & -0.863245 & 2.455336 & 0.000226 \\ 16 & 6 & 0 & 2.139477 & 0.898664 & -0.000112 \\ 17 & 1 & 0 & 2.798702 & 0.850007 & -0.876785 \\ 18 & 1 & 0 & -4.492823 & -0.390780 & 0.879612 \\ 19 & 1 & 0 & 2.678874 & -1.993520 & 0.000130 \\ 20 & 8 & 0 .--196726 & \end{array}$

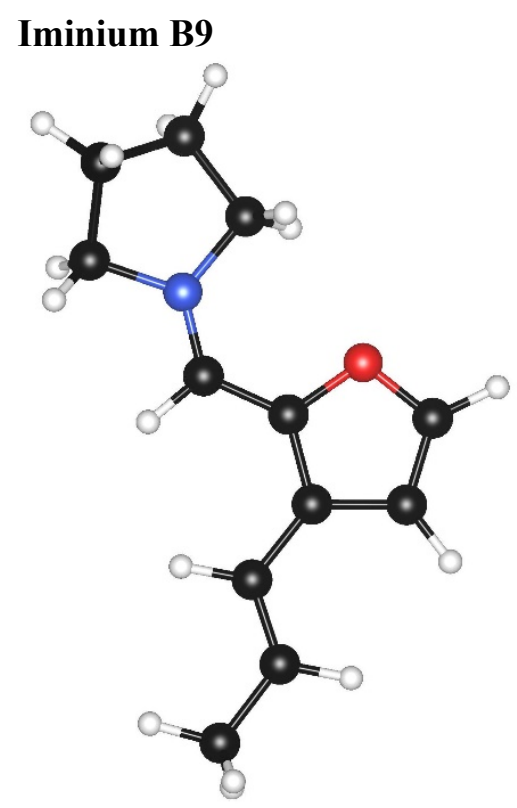

HF $(M 062 X / 6-31+G(d, p))=-596.3955132$ Hartrees

Imaginary Frequencies: none found

Zero-point correction $=0.262114$ (Hartree/Particle)

Thermal correction $=0.220898$ Hartrees

Coordinates from last standard orientation:

\begin{tabular}{|c|c|c|c|c|c|}
\hline \multirow{2}{*}{$\begin{array}{l}\text { Center } \\
\text { Number }\end{array}$} & \multirow{2}{*}{$\begin{array}{l}\text { Atomic } \\
\text { Number }\end{array}$} & \multirow{2}{*}{$\begin{array}{c}\text { Atomic } \\
\text { Type }\end{array}$} & \multicolumn{3}{|c|}{ Coordinates (Angstroms) } \\
\hline & & & $\mathrm{x}$ & $\mathrm{Y}$ & Z \\
\hline 1 & 6 & 0 & -1.767091 & 0.456765 & 0.001594 \\
\hline
\end{tabular}




\begin{tabular}{|c|c|c|c|c|c|}
\hline 2 & 6 & 0 & -0.369878 & 0.497308 & 0.012797 \\
\hline 3 & 6 & 0 & 0.547701 & -0.566714 & 0.037760 \\
\hline 4 & 1 & 0 & 0.117143 & -1.565920 & 0.036943 \\
\hline 5 & 7 & 0 & 1.848244 & -0.508273 & 0.055056 \\
\hline 6 & 6 & 0 & 2.703466 & -1.724094 & 0.048657 \\
\hline 7 & 1 & 0 & 2.276199 & -2.470158 & -0.623574 \\
\hline 8 & 1 & 0 & 2.738157 & -2.126052 & 1.067099 \\
\hline 9 & 6 & 0 & 4.063769 & -1.185160 & -0.383141 \\
\hline 10 & 1 & 0 & 4.106791 & -1.099269 & -1.473419 \\
\hline 11 & 1 & 0 & 4.878321 & -1.832761 & -0.056700 \\
\hline 12 & 6 & 0 & 4.096202 & 0.200080 & 0.270102 \\
\hline 13 & 1 & 0 & 4.837956 & 0.866227 & -0.171957 \\
\hline 14 & 1 & 0 & 4.313208 & 0.109886 & 1.338909 \\
\hline 15 & 6 & 0 & 2.675307 & 0.725484 & 0.065657 \\
\hline 16 & 1 & 0 & 2.327688 & 1.393829 & 0.854162 \\
\hline 17 & 1 & 0 & 2.550528 & 1.226623 & -0.899420 \\
\hline 18 & 6 & 0 & -2.603004 & -0.729608 & 0.043893 \\
\hline 19 & 6 & 0 & -3.943392 & -0.693308 & -0.019969 \\
\hline 20 & 6 & 0 & -4.826074 & -1.894202 & 0.024414 \\
\hline 21 & 1 & 0 & -5.443628 & -1.944686 & -0.878302 \\
\hline 22 & 1 & 0 & -4.251653 & -2.818637 & 0.110616 \\
\hline 23 & 1 & 0 & -2.110727 & -1.695563 & 0.135116 \\
\hline 24 & 1 & 0 & -4.442737 & 0.270897 & -0.111794 \\
\hline 25 & 6 & 0 & -1.033801 & 2.567152 & -0.072601 \\
\hline 26 & 1 & 0 & -0.861297 & 3.633093 & -0.111323 \\
\hline 27 & 6 & 0 & -2.174581 & 1.821863 & -0.053799 \\
\hline 28 & 1 & 0 & -3.181258 & 2.210601 & -0.073046 \\
\hline 29 & 1 & 0 & -5.515101 & -1.826764 & 0.872843 \\
\hline 30 & 8 & 0 & 0.063870 & 1.796646 & -0.031717 \\
\hline
\end{tabular}

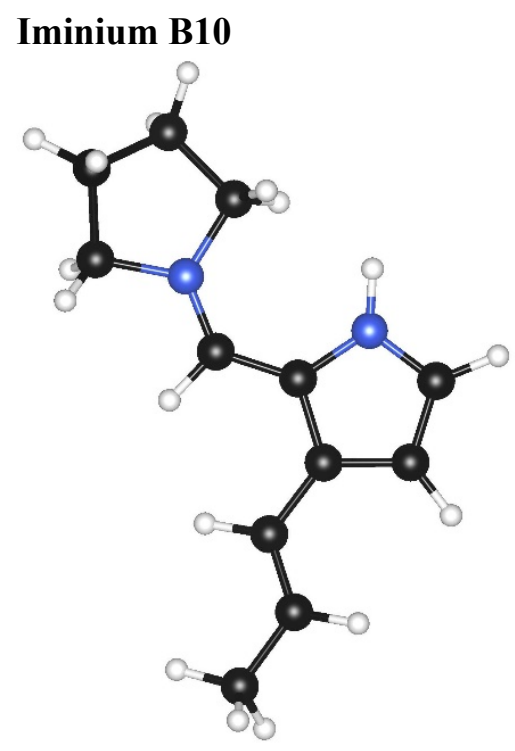

HF $(M 062 X / 6-31+G(d, p))=-576.5496683$ Hartrees

Imaginary Frequencies: none found Zero-point correction $=0.275235$ (Hartree/Particle) Thermal correction $=0.234419$ Hartrees

Coordinates from last standard orientation: 


\begin{tabular}{|c|c|c|c|c|c|}
\hline \multirow{2}{*}{$\begin{array}{l}\text { Center } \\
\text { Number }\end{array}$} & \multirow{2}{*}{$\begin{array}{l}\text { Atomic } \\
\text { Number }\end{array}$} & \multirow{2}{*}{$\begin{array}{c}\text { Atomic } \\
\text { Type }\end{array}$} & \multicolumn{3}{|c|}{ Coordinates (Angstroms) } \\
\hline & & & $\mathrm{x}$ & Y & z \\
\hline 1 & 6 & 0 & -1.778452 & 0.496202 & 0.043162 \\
\hline 2 & 6 & 0 & -0.360193 & 0.610650 & 0.009567 \\
\hline 3 & 6 & 0 & 0.533384 & -0.465978 & 0.012017 \\
\hline 4 & 1 & 0 & 0.074008 & -1.451744 & -0.005576 \\
\hline 5 & 7 & 0 & 1.843218 & -0.473223 & 0.024678 \\
\hline 6 & 6 & 0 & 2.636290 & -1.728588 & -0.010822 \\
\hline 7 & 1 & 0 & 2.196220 & -2.422490 & -0.729016 \\
\hline 8 & 1 & 0 & 2.622478 & -2.179653 & 0.987558 \\
\hline 9 & 6 & 0 & 4.033451 & -1.242039 & -0.384665 \\
\hline 10 & 1 & 0 & 4.114336 & -1.123409 & -1.469718 \\
\hline 11 & 1 & 0 & 4.807651 & -1.936456 & -0.056259 \\
\hline 12 & 6 & 0 & 4.115499 & 0.118743 & 0.314865 \\
\hline 13 & 1 & 0 & 4.897180 & 0.764373 & -0.086969 \\
\hline 14 & 1 & 0 & 4.298461 & -0.014760 & 1.385210 \\
\hline 15 & 6 & 0 & 2.720681 & 0.710802 & 0.095855 \\
\hline 16 & 1 & 0 & 2.401271 & 1.356913 & 0.919241 \\
\hline 17 & 1 & 0 & 2.662293 & 1.253061 & -0.856490 \\
\hline 18 & 6 & 0 & -2.534708 & -0.745728 & 0.141124 \\
\hline 19 & 6 & 0 & -3.854294 & -0.832774 & -0.082415 \\
\hline 20 & 6 & 0 & -4.651104 & -2.090237 & 0.025967 \\
\hline 21 & 1 & 0 & -5.147303 & -2.312452 & -0.924298 \\
\hline 22 & 1 & 0 & -4.027996 & -2.942501 & 0.305057 \\
\hline 23 & 1 & 0 & -1.998400 & -1.651081 & 0.417971 \\
\hline 24 & 1 & 0 & -4.402327 & 0.064570 & -0.368923 \\
\hline 25 & 6 & 0 & -1.212382 & 2.673713 & -0.093938 \\
\hline 26 & 1 & 0 & -1.199752 & 3.752988 & -0.156469 \\
\hline 27 & 6 & 0 & -2.288823 & 1.805059 & -0.018979 \\
\hline 28 & 1 & 0 & -3.326287 & 2.101851 & 0.010389 \\
\hline 29 & 1 & 0 & -5.441494 & -1.975098 & 0.774979 \\
\hline 30 & 7 & 0 & -0.063075 & 1.969610 & -0.069076 \\
\hline 31 & 1 & 0 & 0.852561 & 2.382215 & -0.146331 \\
\hline
\end{tabular}

\section{Iminium B11}

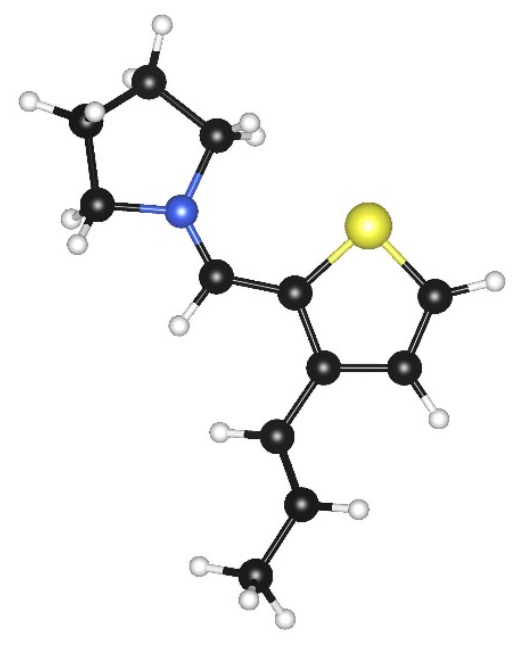

HF $(M 062 X / 6-31+G(d, p))=-919.3607663$ Hartrees Imaginary Frequencies: none found 
Zero-point correction $=0.258647$ (Hartree/Particle)

Thermal correction $=0.217046$ Hartrees

Coordinates from last standard orientation:

\begin{tabular}{|c|c|c|c|c|c|}
\hline \multirow{2}{*}{$\begin{array}{l}\text { Center } \\
\text { Number }\end{array}$} & \multirow{2}{*}{$\begin{array}{l}\text { Atomic } \\
\text { Number }\end{array}$} & \multirow{2}{*}{$\begin{array}{c}\text { Atomic } \\
\text { Type }\end{array}$} & \multicolumn{3}{|c|}{ Coordinates (Angstroms) } \\
\hline & & & $\mathrm{x}$ & Y & Z \\
\hline 1 & 6 & 0 & -1.762529 & 0.325871 & 0.097154 \\
\hline 2 & 6 & 0 & -0.362399 & 0.482490 & 0.069375 \\
\hline 3 & 6 & 0 & 0.525181 & -0.617074 & 0.047949 \\
\hline 4 & 1 & 0 & 0.060778 & -1.601353 & 0.015090 \\
\hline 5 & 7 & 0 & 1.827730 & -0.633629 & 0.044344 \\
\hline 6 & 6 & 0 & 2.608753 & -1.898288 & -0.022249 \\
\hline 7 & 1 & 0 & 2.141439 & -2.581621 & -0.733093 \\
\hline 8 & 1 & 0 & 2.611871 & -2.352525 & 0.974547 \\
\hline 9 & 6 & 0 & 4.002220 & -1.423292 & -0.422398 \\
\hline 10 & 1 & 0 & 4.059513 & -1.291025 & -1.507319 \\
\hline 11 & 1 & 0 & 4.773986 & -2.132585 & -0.121044 \\
\hline 12 & 6 & 0 & 4.114400 & -0.073643 & 0.293345 \\
\hline 13 & 1 & 0 & 4.895931 & 0.567676 & -0.115426 \\
\hline 14 & 1 & 0 & 4.316714 & -0.222908 & 1.358200 \\
\hline 15 & 6 & 0 & 2.725948 & 0.541229 & 0.105548 \\
\hline 16 & 1 & 0 & 2.421669 & 1.194211 & 0.927459 \\
\hline 17 & 1 & 0 & 2.646778 & 1.088526 & -0.840788 \\
\hline 18 & 6 & 0 & -2.459654 & -0.951221 & 0.235010 \\
\hline 19 & 6 & 0 & -3.727384 & -1.149250 & -0.159370 \\
\hline 20 & 6 & 0 & -4.467947 & -2.435907 & -0.009555 \\
\hline 21 & 1 & 0 & -4.798925 & -2.803122 & -0.986321 \\
\hline 22 & 1 & 0 & -3.856240 & -3.204166 & 0.467855 \\
\hline 23 & 1 & 0 & -1.927002 & -1.780202 & 0.695661 \\
\hline 24 & 1 & 0 & -4.268724 & -0.331192 & -0.634229 \\
\hline 25 & 6 & 0 & -1.550305 & 2.634189 & -0.097167 \\
\hline 26 & 1 & 0 & -1.800906 & 3.685342 & -0.166173 \\
\hline 27 & 6 & 0 & -2.418945 & 1.581290 & 0.002836 \\
\hline 28 & 1 & 0 & -3.493236 & 1.706542 & 0.042960 \\
\hline 29 & 1 & 0 & -5.370940 & -2.283670 & 0.591047 \\
\hline 30 & 16 & 0 & 0.089321 & 2.167444 & -0.070106 \\
\hline
\end{tabular}

\section{Trienamine B12}




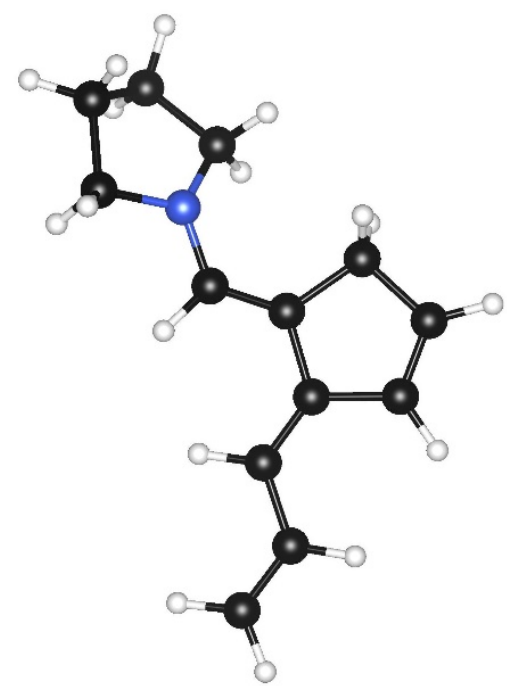

HF $(M 062 X / 6-31+G(d, p))=-560.0733681$ Hartrees

Imaginary Frequencies: none found

Zero-point correction $=0.271641$ (Hartree/Particle)

Thermal correction $=0.230718$ Hartrees

Coordinates from last standard orientation:

\begin{tabular}{|c|c|c|c|c|c|}
\hline \multirow{2}{*}{$\begin{array}{l}\text { Center } \\
\text { Number }\end{array}$} & \multirow{2}{*}{$\begin{array}{l}\text { Atomic } \\
\text { Number }\end{array}$} & \multirow{2}{*}{$\begin{array}{c}\text { Atomic } \\
\text { Type }\end{array}$} & \multicolumn{3}{|c|}{ Coordinates (Angstroms) } \\
\hline & & & $\mathrm{x}$ & $\mathrm{Y}$ & $\mathrm{Z}$ \\
\hline 1 & 6 & 0 & -1.844520 & 0.325597 & -0.027217 \\
\hline 2 & 6 & 0 & -0.384984 & 0.529063 & -0.058253 \\
\hline 3 & 6 & 0 & 0.513622 & -0.460181 & -0.257109 \\
\hline 4 & 1 & 0 & 0.150336 & -1.471389 & -0.447774 \\
\hline 5 & 7 & 0 & 1.879807 & -0.338735 & -0.278135 \\
\hline 6 & 6 & 0 & 2.712463 & -1.494572 & -0.604432 \\
\hline 7 & 1 & 0 & 2.544915 & -1.813400 & -1.637772 \\
\hline 8 & 1 & 0 & 2.485642 & -2.345316 & 0.059507 \\
\hline 9 & 6 & 0 & 4.129508 & -0.980829 & -0.362055 \\
\hline 10 & 1 & 0 & 4.468233 & -0.404485 & -1.229743 \\
\hline 11 & 1 & 0 & 4.844229 & -1.787065 & -0.183152 \\
\hline 12 & 6 & 0 & 3.939353 & -0.058403 & 0.846672 \\
\hline 13 & 1 & 0 & 4.754011 & 0.656899 & 0.979887 \\
\hline 14 & 1 & 0 & 3.861398 & -0.659028 & 1.759360 \\
\hline 15 & 6 & 0 & 2.597354 & 0.627234 & 0.559192 \\
\hline 16 & 1 & 0 & 2.025501 & 0.825854 & 1.473207 \\
\hline 17 & 1 & 0 & 2.740584 & 1.580634 & 0.034743 \\
\hline 18 & 6 & 0 & -2.512780 & -0.842155 & 0.144514 \\
\hline 19 & 6 & 0 & -3.954495 & -0.994414 & 0.100741 \\
\hline 20 & 6 & 0 & -4.596189 & -2.159559 & 0.282093 \\
\hline 21 & 1 & 0 & -5.677556 & -2.224829 & 0.237788 \\
\hline 22 & 1 & 0 & -4.049259 & -3.078233 & 0.478606 \\
\hline 23 & 1 & 0 & -1.942082 & -1.748912 & 0.341764 \\
\hline 24 & 1 & 0 & -4.546117 & -0.102127 & -0.097087 \\
\hline 25 & 6 & 0 & -1.486858 & 2.608780 & -0.217529 \\
\hline 26 & 1 & 0 & -1.666828 & 3.674870 & -0.304708 \\
\hline 27 & 6 & 0 & -2.437628 & 1.663904 & -0.186653 \\
\hline 28 & 1 & 0 & -3.502871 & 1.853790 & -0.242282 \\
\hline 29 & 6 & 0 & -0.104400 & 2.022009 & -0.082396 \\
\hline
\end{tabular}




$\begin{array}{rrrrrr}30 & 1 & 0 & 0.542653 & 2.295941 & -0.926624 \\ 31 & 1 & 0 & 0.385880 & 2.399097 & 0.825819\end{array}$

\section{Fulvene trienamine B12'}

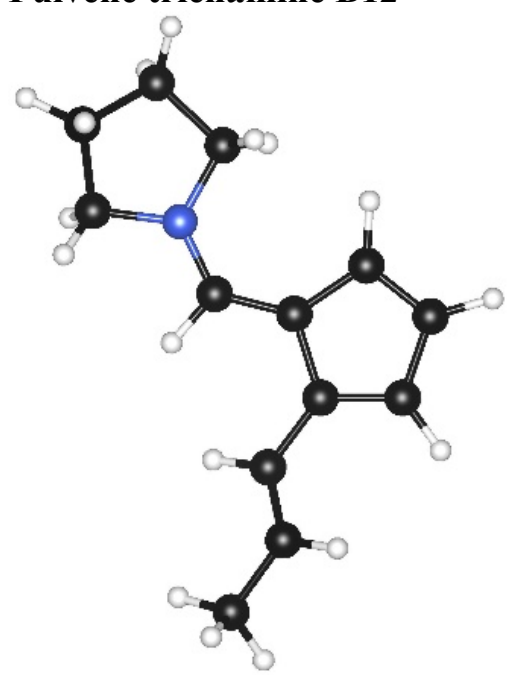

HF $(M 062 X / 6-31+G(d, p))=-560.0894673$ Hartrees

Imaginary Frequencies: none found

Zero-point correction $=0.272227$ (Hartree/Particle)

Thermal correction $=0.232627$ Hartrees

Coordinates from last standard orientation:

\begin{tabular}{|c|c|c|c|c|c|}
\hline \multirow{2}{*}{$\begin{array}{l}\text { Center } \\
\text { Number }\end{array}$} & \multirow{2}{*}{$\begin{array}{l}\text { Atomic } \\
\text { Number }\end{array}$} & \multirow{2}{*}{$\begin{array}{c}\text { Atomic } \\
\text { Type }\end{array}$} & \multicolumn{3}{|c|}{ Coordinates (Angstroms) } \\
\hline & & & $x$ & $Y$ & Z \\
\hline 1 & 6 & 0 & -1.795741 & 0.569478 & 0.117825 \\
\hline 2 & 6 & 0 & -0.336929 & 0.712185 & 0.067526 \\
\hline 3 & 6 & 0 & 0.492483 & -0.382459 & 0.096032 \\
\hline 4 & 1 & 0 & 0.022265 & -1.363529 & 0.148795 \\
\hline 5 & 7 & 0 & 1.831082 & -0.444608 & 0.060162 \\
\hline 6 & 6 & 0 & 2.566638 & -1.711555 & 0.081065 \\
\hline 7 & 1 & 0 & 2.078433 & -2.445710 & -0.565879 \\
\hline 8 & 1 & 0 & 2.606695 & -2.116386 & 1.102150 \\
\hline 9 & 6 & 0 & 3.958496 & -1.308369 & -0.402362 \\
\hline 10 & 1 & 0 & 3.973413 & -1.266496 & -1.496576 \\
\hline 11 & 1 & 0 & 4.733610 & -2.002997 & -0.072275 \\
\hline 12 & 6 & 0 & 4.117621 & 0.100372 & 0.183151 \\
\hline 13 & 1 & 0 & 4.889638 & 0.689904 & -0.315059 \\
\hline 14 & 1 & 0 & 4.372934 & 0.034983 & 1.246151 \\
\hline 15 & 6 & 0 & 2.721901 & 0.712049 & 0.020124 \\
\hline 16 & 1 & 0 & 2.466379 & 1.412312 & 0.822162 \\
\hline 17 & 1 & 0 & 2.608139 & 1.234091 & -0.939524 \\
\hline 18 & 6 & 0 & -2.525836 & -0.692119 & 0.257233 \\
\hline 19 & 6 & 0 & -3.744172 & -0.933834 & -0.244963 \\
\hline 20 & 6 & 0 & -4.510629 & -2.206380 & -0.041179 \\
\hline 21 & 1 & 0 & -4.761658 & -2.676445 & -0.998742 \\
\hline 22 & 1 & 0 & -3.934690 & -2.922641 & 0.552151 \\
\hline 23 & 1 & 0 & -2.048170 & -1.489880 & 0.830228 \\
\hline
\end{tabular}




\begin{tabular}{rrrrrr}
24 & 1 & 0 & -4.222762 & -0.159312 & -0.844524 \\
25 & 6 & 0 & -1.262310 & 2.789185 & -0.100948 \\
26 & 1 & 0 & -1.394539 & 3.860487 & -0.193786 \\
27 & 6 & 0 & -2.331758 & 1.829158 & 0.023122 \\
28 & 1 & 0 & -3.387865 & 2.066894 & 0.071237 \\
29 & 6 & 0 & -0.061485 & 2.134645 & -0.071487 \\
30 & 1 & 0 & 0.908067 & 2.602495 & -0.157402 \\
31 & 1 & 0 & -5.457140 & -2.019646 & 0.478915 \\
\hline
\end{tabular}

\section{Trienamine B13}

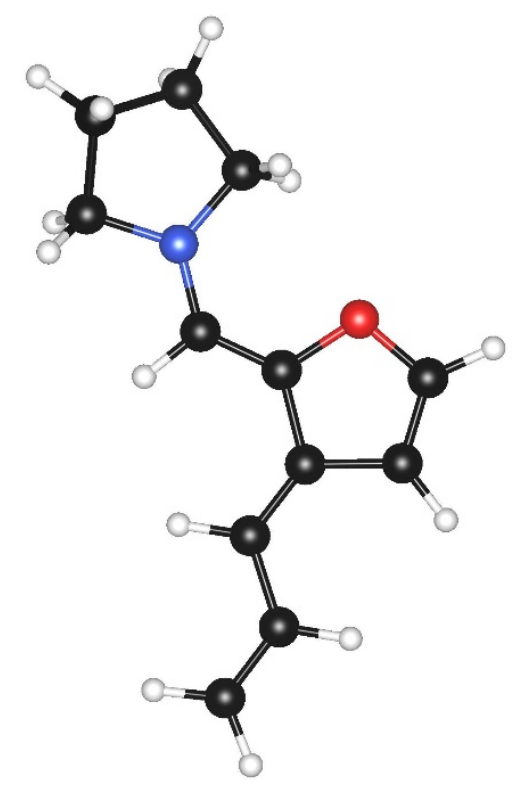

HF $(M 062 X / 6-31+G(d, p))=-595.9714135$ Hartrees

Imaginary Frequencies: none found

Zero-point correction $=0.247927$ (Hartree/Particle)

Thermal correction $=0.206796$ Hartrees

Coordinates from last standard orientation:

\begin{tabular}{|c|c|c|c|c|c|}
\hline \multirow{2}{*}{$\begin{array}{l}\text { Center } \\
\text { Number }\end{array}$} & \multirow{2}{*}{$\begin{array}{l}\text { Atomic } \\
\text { Number }\end{array}$} & \multirow{2}{*}{$\begin{array}{c}\text { Atomic } \\
\text { Type }\end{array}$} & \multicolumn{3}{|c|}{ Coordinates (Angstroms) } \\
\hline & & & $x$ & Y & Z \\
\hline 1 & 6 & 0 & 1.865679 & 0.326327 & 0.003330 \\
\hline 2 & 6 & 0 & 0.405487 & 0.417240 & -0.010400 \\
\hline 3 & 6 & 0 & -0.517025 & -0.572835 & -0.020034 \\
\hline 4 & 1 & 0 & -0.128063 & -1.587450 & -0.020391 \\
\hline 5 & 7 & 0 & -1.872309 & -0.484378 & -0.020135 \\
\hline 6 & 6 & 0 & -2.722744 & -1.670594 & 0.014291 \\
\hline 7 & 1 & 0 & -2.356519 & -2.388546 & 0.755681 \\
\hline 8 & 1 & 0 & -2.745244 & -2.169892 & -0.966794 \\
\hline 9 & 6 & 0 & -4.095207 & -1.098990 & 0.367440 \\
\hline 10 & 1 & 0 & -4.174565 & -0.969978 & 1.452313 \\
\hline 11 & 1 & 0 & -4.914936 & -1.739843 & 0.035520 \\
\hline 12 & 6 & 0 & -4.073739 & 0.268499 & -0.325257 \\
\hline 13 & 1 & 0 & -4.818654 & 0.963760 & 0.067501 \\
\hline
\end{tabular}




\begin{tabular}{|c|c|c|c|c|c|}
\hline 14 & 1 & 0 & -4.256455 & 0.142972 & -1.398100 \\
\hline 15 & 6 & 0 & -2.640477 & 0.758531 & -0.095623 \\
\hline 16 & 1 & 0 & -2.272944 & 1.392860 & -0.906557 \\
\hline 17 & 1 & 0 & -2.546720 & 1.324151 & 0.840538 \\
\hline 18 & 6 & 0 & 2.637806 & -0.793134 & -0.048221 \\
\hline 19 & 6 & 0 & 4.082137 & -0.791347 & -0.001103 \\
\hline 20 & 6 & 0 & 4.852725 & -1.891467 & -0.055484 \\
\hline 21 & 1 & 0 & 5.934057 & -1.828115 & -0.013245 \\
\hline 22 & 1 & 0 & 4.414071 & -2.882072 & -0.143771 \\
\hline 23 & 1 & 0 & 2.154955 & -1.765665 & -0.129787 \\
\hline 24 & 1 & 0 & 4.571720 & 0.177748 & 0.088403 \\
\hline 25 & 8 & 0 & 0.031441 & 1.766185 & 0.030357 \\
\hline 26 & 6 & 0 & 1.184754 & 2.481773 & 0.088771 \\
\hline 27 & 1 & 0 & 1.055982 & 3.554355 & 0.129784 \\
\hline 28 & 6 & 0 & 2.292532 & 1.723508 & 0.081011 \\
\hline 29 & 1 & 0 & 3.306382 & 2.091808 & 0.114660 \\
\hline
\end{tabular}

\section{Trienamine B14}

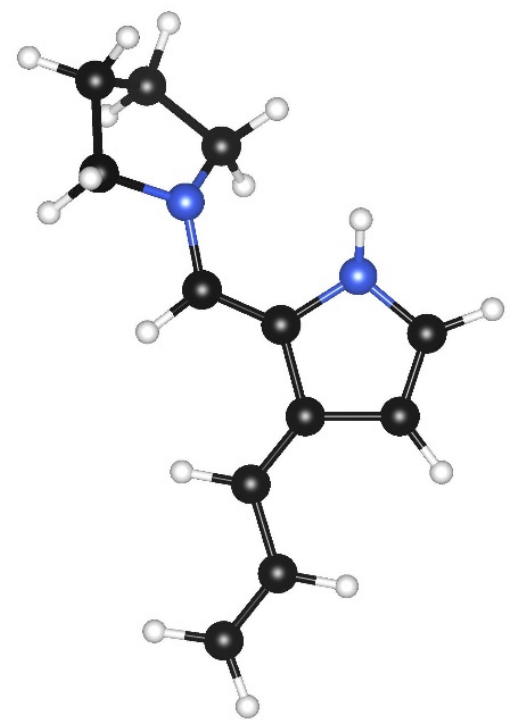

HF $(M 062 X / 6-31+G(d, p))=-576.1149762$ Hartrees

Imaginary Frequencies: none found

Zero-point correction $=0.260139$ (Hartree/Particle)

Thermal correction $=0.219492$ Hartrees

Coordinates from last standard orientation:

\begin{tabular}{cccccc} 
Center & Atomic & Atomic & \multicolumn{3}{c}{ Coordinates (Angstroms) } \\
Number & Number & Type & $X$ & Y & $Z$ \\
-1 & 6 & 0 & -1.857374 & 0.329637 & -0.069101 \\
2 & 6 & 0 & -0.394237 & 0.471413 & -0.174996 \\
3 & 6 & 0 & 0.540909 & -0.484268 & -0.367254 \\
4 & 1 & 0 & 0.220975 & -1.506911 & -0.561141 \\
5 & 7 & 0 & 1.905771 & -0.250295 & -0.374043 \\
6 & 6 & 0 & 2.790119 & -1.346045 & -0.770950 \\
7 & 1 & 0 & 2.758002 & -1.498136 & -1.854220 \\
8 & 1 & 0 & 2.486219 & -2.288115 & -0.283660
\end{tabular}




$\begin{array}{rrrrrr}9 & 6 & 0 & 4.161611 & -0.901258 & -0.268604 \\ 10 & 1 & 0 & 4.609212 & -0.195962 & -0.977415 \\ 11 & 1 & 0 & 4.850425 & -1.738276 & -0.135080 \\ 12 & 6 & 0 & 3.808419 & -0.187359 & 1.040031 \\ 13 & 1 & 0 & 4.599550 & 0.471970 & 1.404727 \\ 14 & 1 & 0 & 3.595367 & -0.929007 & 1.817740 \\ 15 & 6 & 0 & 2.526780 & 0.571898 & 0.681584 \\ 16 & 1 & 0 & 1.838440 & 0.682876 & 1.525516 \\ 17 & 1 & 0 & 2.765195 & 1.577550 & 0.308714 \\ 18 & 6 & 0 & -2.547372 & -0.830942 & 0.094632 \\ 19 & 6 & 0 & -3.989503 & -0.922797 & 0.156479 \\ 20 & 6 & 0 & -4.674066 & -2.068547 & 0.313091 \\ 21 & 1 & 0 & -5.757423 & -2.080904 & 0.351560 \\ 22 & 1 & 0 & -4.161033 & -3.022500 & 0.404668 \\ 23 & 1 & 0 & -1.993463 & -1.762776 & 0.196688 \\ 24 & 1 & 0 & -4.548124 & 0.007819 & 0.065591 \\ 25 & 6 & 0 & -1.311050 & 2.540073 & -0.175460 \\ 26 & 1 & 0 & -1.309558 & 3.621631 & -0.215535 \\ 27 & 6 & 0 & -2.367385 & 1.699652 & -0.124698 \\ 28 & 1 & 0 & -3.403651 & 1.998590 & -0.074287 \\ 29 & 7 & 0 & -0.102148 & 1.855207 & -0.107269 \\ 30 & 1 & 0 & 0.703385 & 2.199012 & -0.613207 \\ ---------------------1\end{array}$

Fulvene trienamine B14' 


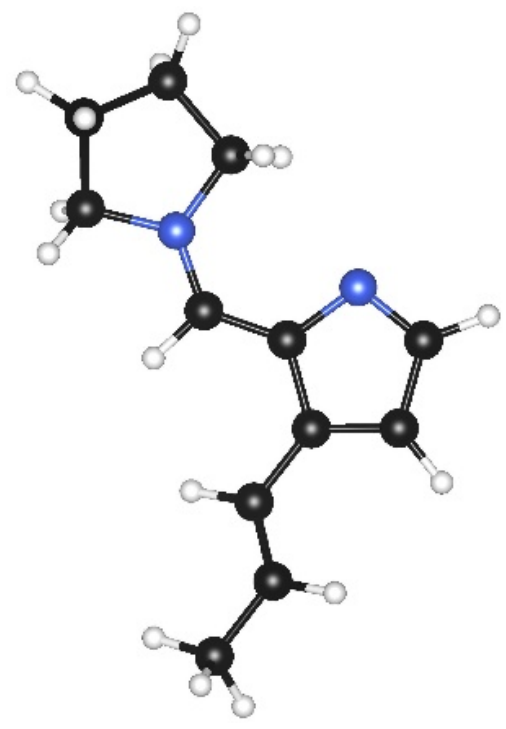

HF $(M 062 X / 6-31+G(d, p))=-576.1430189$ Hartrees

Imaginary Frequencies: none found

Zero-point correction $=0.260901$ (Hartree/Particle)

Thermal correction $=0.219145$ Hartrees

Coordinates from last standard orientation:

\begin{tabular}{|c|c|c|c|c|c|}
\hline \multirow{2}{*}{$\begin{array}{l}\text { Center } \\
\text { Number }\end{array}$} & \multirow{2}{*}{$\begin{array}{l}\text { Atomic } \\
\text { Number }\end{array}$} & \multirow{2}{*}{$\begin{array}{c}\text { Atomic } \\
\text { Type }\end{array}$} & \multicolumn{3}{|c|}{ Coordinates (Angstroms) } \\
\hline & & & $\mathrm{X}$ & $\mathrm{Y}$ & $\mathrm{z}$ \\
\hline 1 & 6 & 0 & -1.789362 & 0.552208 & 0.096862 \\
\hline 2 & 6 & 0 & -0.341784 & 0.632152 & 0.077758 \\
\hline 3 & 6 & 0 & 0.504137 & -0.458528 & 0.124022 \\
\hline 4 & 1 & 0 & 0.050605 & -1.446070 & 0.190651 \\
\hline 5 & 7 & 0 & 1.833659 & -0.472131 & 0.089602 \\
\hline 6 & 6 & 0 & 2.615041 & -1.712988 & 0.130982 \\
\hline 7 & 1 & 0 & 2.131969 & -2.486253 & -0.472772 \\
\hline 8 & 1 & 0 & 2.696542 & -2.075106 & 1.164979 \\
\hline 9 & 6 & 0 & 3.978599 & -1.280416 & -0.404470 \\
\hline 10 & 1 & 0 & 3.961237 & -1.272819 & -1.499510 \\
\hline 11 & 1 & 0 & 4.783643 & -1.940981 & -0.076028 \\
\hline 12 & 6 & 0 & 4.104460 & 0.150419 & 0.133226 \\
\hline 13 & 1 & 0 & 4.846266 & 0.746292 & -0.402044 \\
\hline 14 & 1 & 0 & 4.388768 & 0.126321 & 1.190923 \\
\hline 15 & 6 & 0 & 2.690793 & 0.718239 & -0.017802 \\
\hline 16 & 1 & 0 & 2.412950 & 1.449492 & 0.742992 \\
\hline 17 & 1 & 0 & 2.522357 & 1.193380 & -0.990573 \\
\hline 18 & 6 & 0 & -2.592555 & -0.666063 & 0.196777 \\
\hline 19 & 6 & 0 & -3.866376 & -0.781219 & -0.200290 \\
\hline 20 & 6 & 0 & -4.691431 & -2.024430 & -0.060991 \\
\hline 21 & 1 & 0 & -5.051206 & -2.372133 & -1.035724 \\
\hline 22 & 1 & 0 & -4.115368 & -2.831574 & 0.400446 \\
\hline 23 & 1 & 0 & -2.115742 & -1.544376 & 0.635521 \\
\hline 24 & 1 & 0 & -4.348931 & 0.080063 & -0.662350 \\
\hline 25 & 6 & 0 & -1.027134 & 2.659903 & -0.076715 \\
\hline 26 & 1 & 0 & -0.989100 & 3.741716 & -0.157631 \\
\hline 27 & 6 & 0 & -2.213538 & 1.860388 & 0.000545 \\
\hline 28 & 1 & 0 & -3.233669 & 2.221598 & 0.013549 \\
\hline
\end{tabular}




\begin{tabular}{|c|c|c|c|c|c|}
\hline 29 & 7 & 0 & 0.082299 & 1.951277 & -0.032435 \\
\hline 30 & 1 & 0 & -5.577122 & -1.841564 & 0.557992 \\
\hline
\end{tabular}

\section{Trienamine B15}

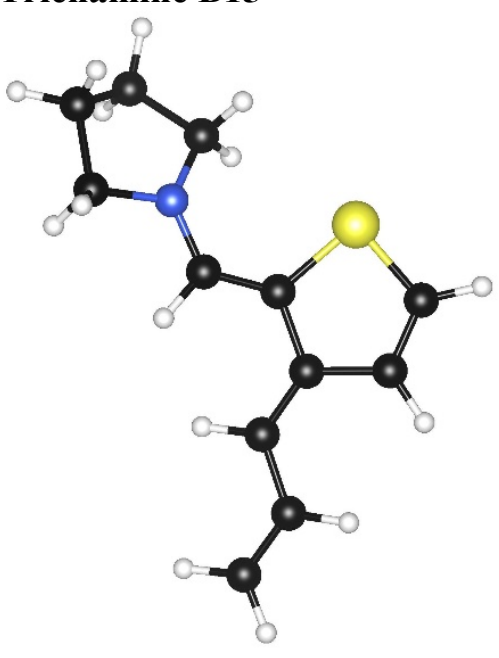

HF $(M 062 X / 6-31+G(d, p))=-918.9390192$ Hartrees

Imaginary Frequencies: none found

Zero-point correction $=0.245074$ (Hartree/Particle)

Thermal correction $=0.203768$ Hartrees

Coordinates from last standard orientation:

\begin{tabular}{|c|c|c|c|c|c|}
\hline \multirow{2}{*}{$\begin{array}{l}\text { Center } \\
\text { Number }\end{array}$} & \multirow{2}{*}{$\begin{array}{l}\text { Atomic } \\
\text { Number }\end{array}$} & \multirow{2}{*}{$\begin{array}{c}\text { Atomic } \\
\text { Type }\end{array}$} & \multicolumn{3}{|c|}{ Coordinates (Angstroms) } \\
\hline & & & $\mathrm{X}$ & Y & Z \\
\hline & & & & & \\
\hline 1 & 6 & 0 & -1.844627 & 0.167030 & -0.029628 \\
\hline 2 & 6 & 0 & -0.389359 & 0.382493 & -0.051990 \\
\hline 3 & 6 & 0 & 0.526010 & -0.595573 & -0.269111 \\
\hline 4 & 1 & 0 & 0.145198 & -1.590272 & -0.503503 \\
\hline 5 & 7 & 0 & 1.883339 & -0.500346 & -0.256770 \\
\hline 6 & 6 & 0 & 2.712168 & -1.645820 & -0.633664 \\
\hline 7 & 1 & 0 & 2.551584 & -1.914156 & -1.682370 \\
\hline 8 & 1 & 0 & 2.466974 & -2.521194 & -0.011944 \\
\hline 9 & 6 & 0 & 4.133697 & -1.157112 & -0.357793 \\
\hline 10 & 1 & 0 & 4.502131 & -0.583167 & -1.214835 \\
\hline 11 & 1 & 0 & 4.828206 & -1.978773 & -0.170488 \\
\hline 12 & 6 & 0 & 3.941733 & -0.234441 & 0.850376 \\
\hline 13 & 1 & 0 & 4.772614 & 0.457444 & 1.004030 \\
\hline 14 & 1 & 0 & 3.819960 & -0.832512 & 1.760095 \\
\hline 15 & 6 & 0 & 2.631196 & 0.488791 & 0.524880 \\
\hline 16 & 1 & 0 & 2.061174 & 0.764655 & 1.418452 \\
\hline 17 & 1 & 0 & 2.815915 & 1.399486 & -0.059094 \\
\hline 18 & 6 & 0 & -2.451657 & -1.036818 & 0.167918 \\
\hline 19 & 6 & 0 & -3.876687 & -1.278189 & 0.081076 \\
\hline 20 & 6 & 0 & -4.454278 & -2.471978 & 0.295324 \\
\hline 21 & 1 & 0 & -5.527436 & -2.604531 & 0.217090 \\
\hline 22 & 1 & 0 & -3.861645 & -3.345837 & 0.553623 \\
\hline 23 & 1 & 0 & -1.836144 & -1.897092 & 0.424366 \\
\hline 24 & 1 & 0 & -4.514889 & -0.436010 & -0.180173 \\
\hline
\end{tabular}




$\begin{array}{llllll}25 & 6 & 0 & -1.699716 & 2.511015 & -0.195085 \\ 26 & 1 & 0 & -1.989306 & 3.552177 & -0.261421 \\ 27 & 6 & 0 & -2.524114 & 1.449401 & -0.201789\end{array}$

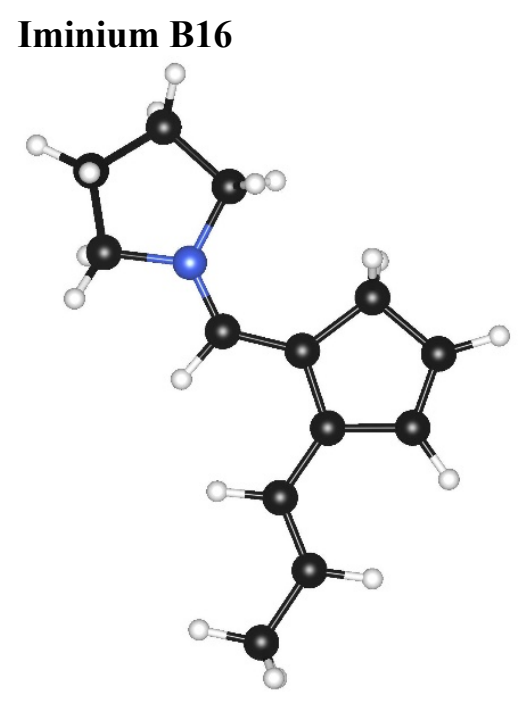

HF $(M 062 X / 6-31+G(d, p))=-560.4863791$ Hartrees

Imaginary Frequencies: none found

Zero-point correction $=0.285357$ (Hartree/Particle)

Thermal correction $=0.243778$ Hartrees

\section{Coordinates from last standard orientation:}

\begin{tabular}{|c|c|c|c|c|c|}
\hline \multirow{2}{*}{$\begin{array}{l}\text { Center } \\
\text { Number }\end{array}$} & \multirow{2}{*}{$\begin{array}{l}\text { Atomic } \\
\text { Number }\end{array}$} & \multirow{2}{*}{$\begin{array}{c}\text { Atomic } \\
\text { Type }\end{array}$} & \multicolumn{3}{|c|}{ Coordinates (Angstroms) } \\
\hline & & & $\mathrm{x}$ & Y & Z \\
\hline 1 & 6 & 0 & -1.739530 & 0.453729 & -0.001317 \\
\hline 2 & 6 & 0 & -0.353124 & 0.631638 & 0.039042 \\
\hline 3 & 6 & 0 & 0.536041 & -0.456997 & 0.102536 \\
\hline 4 & 1 & 0 & 0.100856 & -1.452320 & 0.162249 \\
\hline 5 & 7 & 0 & 1.844733 & -0.456937 & 0.099348 \\
\hline 6 & 6 & 0 & 2.638576 & -1.708606 & 0.171844 \\
\hline 7 & 1 & 0 & 2.139315 & -2.494812 & -0.397342 \\
\hline 8 & 1 & 0 & 2.713674 & -2.013614 & 1.221575 \\
\hline 9 & 6 & 0 & 3.995921 & -1.290757 & -0.382248 \\
\hline 10 & 1 & 0 & 3.977611 & -1.305774 & -1.476563 \\
\hline 11 & 1 & 0 & 4.796095 & -1.949315 & -0.042291 \\
\hline 12 & 6 & 0 & 4.137527 & 0.144339 & 0.136993 \\
\hline 13 & 1 & 0 & 4.878942 & 0.728773 & -0.409083 \\
\hline 14 & 1 & 0 & 4.420559 & 0.137971 & 1.193950 \\
\hline 15 & 6 & 0 & 2.729424 & 0.723059 & -0.024641 \\
\hline 16 & 1 & 0 & 2.479913 & 1.463064 & 0.737353 \\
\hline 17 & 1 & 0 & 2.581340 & 1.162490 & -1.016865 \\
\hline 18 & 6 & 0 & -2.465457 & -0.799850 & -0.002483 \\
\hline 19 & 6 & 0 & -3.808811 & -0.885411 & -0.017673 \\
\hline 20 & 6 & 0 & -4.570793 & -2.165599 & -0.020972 \\
\hline 21 & 1 & 0 & -5.215600 & -2.217840 & -0.904730 \\
\hline 22 & 1 & 0 & -3.911064 & -3.035152 & -0.012679 \\
\hline 23 & 1 & 0 & -1.900452 & -1.728344 & 0.006884 \\
\hline
\end{tabular}




$\begin{array}{rrrrrr}24 & 1 & 0 & -4.404100 & 0.025929 & -0.028017 \\ 25 & 6 & 0 & -1.411284 & 2.721100 & -0.045676 \\ 26 & 1 & 0 & -1.580467 & 3.791020 & -0.076453 \\ 27 & 6 & 0 & -2.364819 & 1.768662 & -0.054149 \\ 28 & 1 & 0 & -3.430666 & 1.947750 & -0.096404 \\ 29 & 6 & 0 & -0.050083 & 2.111690 & 0.017952 \\ 30 & 1 & 0 & 0.556740 & 2.418214 & -0.843098 \\ 31 & 1 & 0 & 0.474775 & 2.452027 & 0.920113 \\ 32 & 1 & 0 & -5.232129 & -2.213497 & 0.850715 \\ -\end{array}$

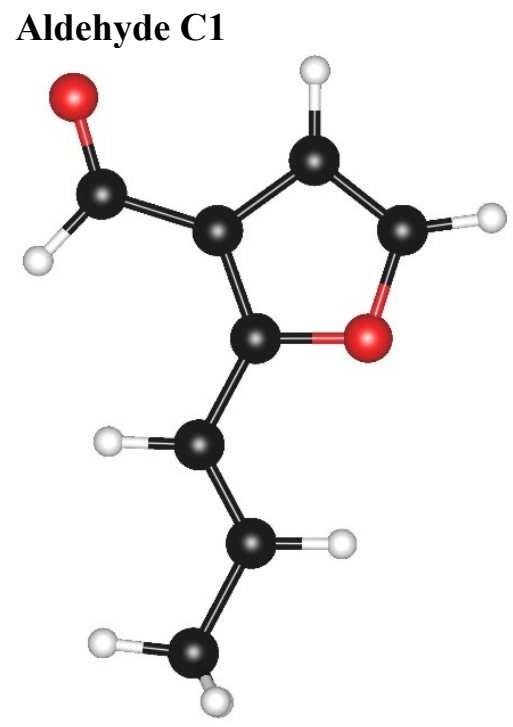

HF $(M 062 X / 6-31+G(d, p))=-459.8997539$ Hartrees Imaginary Frequencies: none found Zero-point correction $=0.142096$ (Hartree/Particle) Thermal correction $=0.106772$ Hartrees

Coordinates from last standard orientation:

\begin{tabular}{|c|c|c|c|c|c|}
\hline \multirow{2}{*}{$\begin{array}{l}\text { Center } \\
\text { Number }\end{array}$} & \multirow{2}{*}{$\begin{array}{l}\text { Atomic } \\
\text { Number }\end{array}$} & \multirow{2}{*}{$\begin{array}{l}\text { Atomic } \\
\text { Type }\end{array}$} & \multicolumn{3}{|c|}{ Coordinates (Angstroms) } \\
\hline & & & $\mathrm{X}$ & $\mathrm{Y}$ & $\mathrm{Z}$ \\
\hline & & & & 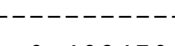 & ---------- \\
\hline 1 & 6 & 0 & -0.806022 & 0.402470 & -0.000384 \\
\hline 2 & 6 & 0 & -1.945642 & -0.312131 & 0.000038 \\
\hline 3 & 6 & 0 & -3.243033 & 0.371508 & -0.000186 \\
\hline 4 & 1 & 0 & -3.189623 & 1.481947 & -0.000992 \\
\hline 5 & 1 & 0 & -1.959956 & -1.398791 & 0.000649 \\
\hline 6 & 1 & 0 & -0.889709 & 1.490258 & -0.000962 \\
\hline 7 & 6 & 0 & 0.536826 & -0.140667 & -0.000173 \\
\hline 8 & 6 & 0 & 1.700695 & 0.587561 & -0.000012 \\
\hline 9 & 6 & 0 & 0.939868 & -1.527476 & -0.000200 \\
\hline 10 & 6 & 0 & 2.291320 & -1.524967 & 0.000031 \\
\hline 11 & 1 & 0 & 0.299992 & -2.397277 & -0.000370 \\
\hline 12 & 1 & 0 & 3.038733 & -2.302341 & 0.000082 \\
\hline 13 & 6 & 0 & 2.003122 & 2.042417 & 0.000189 \\
\hline 14 & 1 & 0 & 2.585516 & 2.314909 & -0.884710 \\
\hline 15 & 1 & 0 & 1.081021 & 2.624798 & 0.000013 \\
\hline 16 & 1 & 0 & 2.585031 & 2.314730 & 0.885463 \\
\hline
\end{tabular}




$\begin{array}{rrrrrr}17 & 8 & 0 & 2.764021 & -0.246351 & 0.000199 \\ 18 & 8 & 0 & -4.315747 & -0.193714 & 0.000427\end{array}$

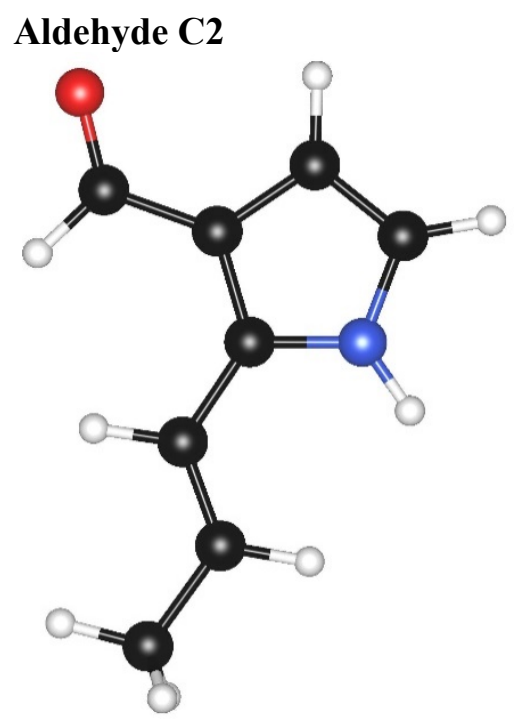

HF $(M 062 X / 6-31+G(d, p))=-440.0527351$ Hartrees

Imaginary Frequencies: none found

Zero-point correction $=0.154786$ (Hartree/Particle $)$

Thermal correction $=0.118401$ Hartrees

Coordinates from last standard orientation:

\begin{tabular}{|c|c|c|c|c|c|}
\hline \multirow{2}{*}{$\begin{array}{l}\text { Center } \\
\text { Number }\end{array}$} & \multirow{2}{*}{$\begin{array}{l}\text { Atomic } \\
\text { Number }\end{array}$} & \multirow{2}{*}{$\begin{array}{c}\text { Atomic } \\
\text { Type }\end{array}$} & \multicolumn{3}{|c|}{ Coordinates (Angstroms) } \\
\hline & & & $X$ & $\mathrm{Y}$ & Z \\
\hline 1 & 6 & 0 & -0.806453 & 0.383111 & -0.000368 \\
\hline 2 & 6 & 0 & -1.950248 & -0.329496 & 0.000082 \\
\hline 3 & 6 & 0 & -3.241031 & 0.358683 & -0.000175 \\
\hline 4 & 1 & 0 & -3.179161 & 1.469390 & -0.000982 \\
\hline 5 & 1 & 0 & -1.967351 & -1.415981 & 0.000729 \\
\hline 6 & 1 & 0 & -0.899120 & 1.470634 & -0.000955 \\
\hline 7 & 6 & 0 & 0.538075 & -0.149235 & -0.000173 \\
\hline 8 & 6 & 0 & 1.703614 & 0.610340 & -0.000011 \\
\hline 9 & 6 & 0 & 0.933147 & -1.528816 & -0.000153 \\
\hline 10 & 6 & 0 & 2.299178 & -1.563585 & 0.000034 \\
\hline 11 & 1 & 0 & 0.278540 & -2.387971 & -0.000312 \\
\hline 12 & 1 & 0 & 2.988368 & -2.393896 & 0.000033 \\
\hline 13 & 6 & 0 & 1.926543 & 2.087293 & 0.000198 \\
\hline 14 & 1 & 0 & 2.484613 & 2.405978 & -0.886344 \\
\hline 15 & 1 & 0 & 0.974723 & 2.619514 & -0.000020 \\
\hline 16 & 1 & 0 & 2.484075 & 2.405785 & 0.887149 \\
\hline 17 & 8 & 0 & -4.321519 & -0.195059 & 0.000373 \\
\hline 18 & 7 & 0 & 2.752514 & -0.262048 & 0.000119 \\
\hline 19 & 1 & 0 & 3.722917 & 0.011588 & 0.000291 \\
\hline
\end{tabular}

\section{Aldehyde C3}




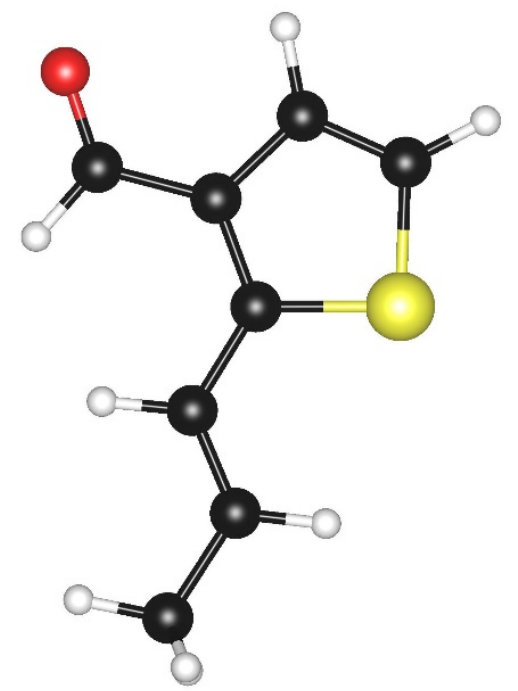

HF $(M 062 X / 6-31+G(d, p))=-782.864751$ Hartrees

Imaginary Frequencies: none found

Zero-point correction $=0.138356$ (Hartree/Particle)

Thermal correction $=0.102913$ Hartrees

Coordinates from last standard orientation:

\begin{tabular}{|c|c|c|c|c|c|}
\hline \multirow{2}{*}{$\begin{array}{l}\text { Center } \\
\text { Number }\end{array}$} & \multirow{2}{*}{$\begin{array}{l}\text { Atomic } \\
\text { Number }\end{array}$} & \multirow{2}{*}{$\begin{array}{c}\text { Atomic } \\
\text { Type }\end{array}$} & \multicolumn{3}{|c|}{ Coordinates (Angstroms) } \\
\hline & & & $\mathrm{x}$ & $\mathrm{Y}$ & Z \\
\hline 1 & 6 & 0 & -1.122570 & 0.413475 & -0.000143 \\
\hline 2 & 6 & 0 & -2.237350 & -0.339552 & 0.000080 \\
\hline 3 & 6 & 0 & -3.556431 & 0.302756 & -0.000062 \\
\hline 4 & 1 & 0 & -3.540218 & 1.414076 & -0.000256 \\
\hline 5 & 1 & 0 & -2.223474 & -1.425818 & 0.000363 \\
\hline 6 & 1 & 0 & -1.255161 & 1.495157 & -0.000404 \\
\hline 7 & 6 & 0 & 0.248157 & -0.077147 & -0.000081 \\
\hline 8 & 6 & 0 & 1.357798 & 0.744430 & 0.000001 \\
\hline 9 & 6 & 0 & 0.604945 & -1.472177 & -0.000100 \\
\hline 10 & 6 & 0 & 1.946522 & -1.675468 & -0.000026 \\
\hline 11 & 1 & 0 & -0.116368 & -2.280122 & -0.000195 \\
\hline 12 & 1 & 0 & 2.481138 & -2.614697 & -0.000022 \\
\hline 13 & 6 & 0 & 1.439914 & 2.241439 & 0.000041 \\
\hline 14 & 1 & 0 & 1.970802 & 2.605536 & -0.884429 \\
\hline 15 & 1 & 0 & 0.445847 & 2.690125 & 0.000016 \\
\hline 16 & 1 & 0 & 1.970726 & 2.605494 & 0.884574 \\
\hline 17 & 8 & 0 & -4.609296 & -0.298304 & 0.000127 \\
\hline 18 & 16 & 0 & 2.815948 & -0.183116 & 0.000068 \\
\hline
\end{tabular}

\section{Enol C4}




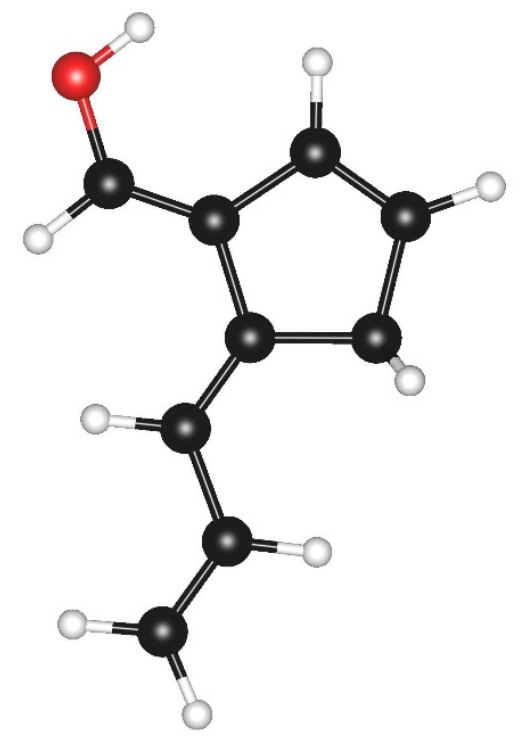

HF $(M 062 X / 6-31+G(d, p))=-423.9631305$ Hartrees

Imaginary Frequencies: none found

Zero-point correction $=0.165237$ (Hartree/Particle)

Thermal correction $=0.129479$ Hartrees

Coordinates from last standard orientation:

\begin{tabular}{|c|c|c|c|c|c|}
\hline \multirow{2}{*}{$\begin{array}{l}\text { Center } \\
\text { Number }\end{array}$} & \multirow{2}{*}{$\begin{array}{l}\text { Atomic } \\
\text { Number }\end{array}$} & \multirow{2}{*}{$\begin{array}{c}\text { Atomic } \\
\text { Type }\end{array}$} & \multicolumn{3}{|c|}{ Coordinates (Angstroms) } \\
\hline & & & $\mathrm{x}$ & Y & Z \\
\hline 1 & 6 & 0 & -0.656707 & 0.529157 & -0.000313 \\
\hline 2 & 6 & 0 & -1.904970 & -0.205457 & -0.000317 \\
\hline 3 & 6 & 0 & -3.097111 & 0.413076 & 0.000091 \\
\hline 4 & 1 & 0 & -3.192027 & 1.495006 & 0.000384 \\
\hline 5 & 1 & 0 & -1.873206 & -1.294198 & -0.000726 \\
\hline 6 & 1 & 0 & -0.740816 & 1.615523 & -0.000736 \\
\hline 7 & 6 & 0 & 0.579237 & -0.015994 & 0.000049 \\
\hline 8 & 6 & 0 & 1.867806 & 0.726034 & -0.000080 \\
\hline 9 & 6 & 0 & 0.926677 & -1.443364 & 0.000400 \\
\hline 10 & 6 & 0 & 2.256356 & -1.617983 & 0.000281 \\
\hline 11 & 1 & 0 & 0.197518 & -2.245675 & 0.000832 \\
\hline 12 & 1 & 0 & 2.761187 & -2.577652 & 0.000545 \\
\hline 13 & 6 & 0 & 2.043536 & 2.050239 & 0.000386 \\
\hline 14 & 1 & 0 & 3.039075 & 2.483475 & 0.000369 \\
\hline 15 & 1 & 0 & 1.206007 & 2.741264 & 0.000859 \\
\hline 16 & 6 & 0 & 2.991926 & -0.302119 & -0.000504 \\
\hline 17 & 1 & 0 & 3.636920 & -0.194995 & 0.880182 \\
\hline 18 & 1 & 0 & 3.635635 & -0.195509 & -0.882217 \\
\hline 19 & 8 & 0 & -4.312699 & -0.185443 & 0.000093 \\
\hline 20 & 1 & 0 & -4.209200 & -1.145234 & -0.000198 \\
\hline
\end{tabular}

\section{Enol C5}




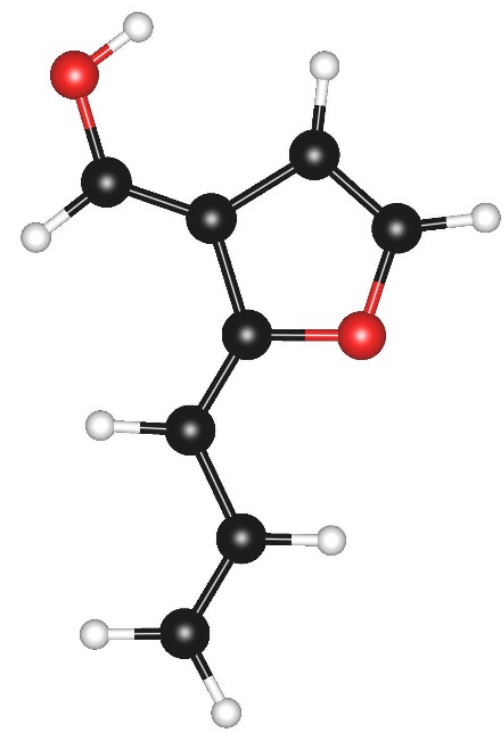

HF $(M 062 X / 6-31+G(d, p))=-459.862049$ Hartrees

Imaginary Frequencies: none found

Zero-point correction $=0.141006$ (Hartree/Particle)

Thermal correction $=0.105386$ Hartrees

Coordinates from last standard orientation:

\begin{tabular}{|c|c|c|c|c|c|}
\hline \multirow{2}{*}{$\begin{array}{l}\text { Center } \\
\text { Number }\end{array}$} & \multirow{2}{*}{$\begin{array}{l}\text { Atomic } \\
\text { Number }\end{array}$} & \multirow{2}{*}{$\begin{array}{c}\text { Atomic } \\
\text { Type }\end{array}$} & \multicolumn{3}{|c|}{ Coordinates (Angstroms) } \\
\hline & & & $x$ & Y & Z \\
\hline 1 & 6 & & & 513107 & ---------- \\
\hline 1 & 6 & 0 & -0.645009 & 0.513107 & -0.000311 \\
\hline 2 & 6 & 0 & -1.889148 & -0.220402 & -0.000467 \\
\hline 3 & 6 & 0 & -3.079553 & 0.397602 & 0.000399 \\
\hline 4 & 1 & 0 & -3.163770 & 1.483292 & 0.001584 \\
\hline 5 & 1 & 0 & -1.874770 & -1.307130 & -0.001285 \\
\hline 6 & 1 & 0 & -0.714583 & 1.600312 & -0.000105 \\
\hline 7 & 6 & 0 & 0.585610 & -0.048214 & -0.000259 \\
\hline 8 & 6 & 0 & 1.872862 & 0.679530 & 0.000000 \\
\hline 9 & 6 & 0 & 0.966719 & -1.459056 & -0.000134 \\
\hline 10 & 8 & 0 & 2.891284 & -0.263280 & 0.000422 \\
\hline 11 & 6 & 0 & 2.306368 & -1.498904 & 0.000336 \\
\hline 12 & 1 & 0 & 0.308009 & -2.314029 & -0.000207 \\
\hline 13 & 1 & 0 & 2.997658 & -2.329425 & 0.000655 \\
\hline 14 & 6 & 0 & 2.164720 & 1.982479 & -0.000216 \\
\hline 15 & 1 & 0 & 3.194700 & 2.315261 & 0.000010 \\
\hline 16 & 1 & 0 & 1.373638 & 2.721001 & -0.000711 \\
\hline 17 & 8 & 0 & -4.243545 & -0.303973 & -0.000742 \\
\hline 18 & 1 & 0 & -4.998215 & 0.291887 & 0.006533 \\
\hline
\end{tabular}

\section{Enol C6}




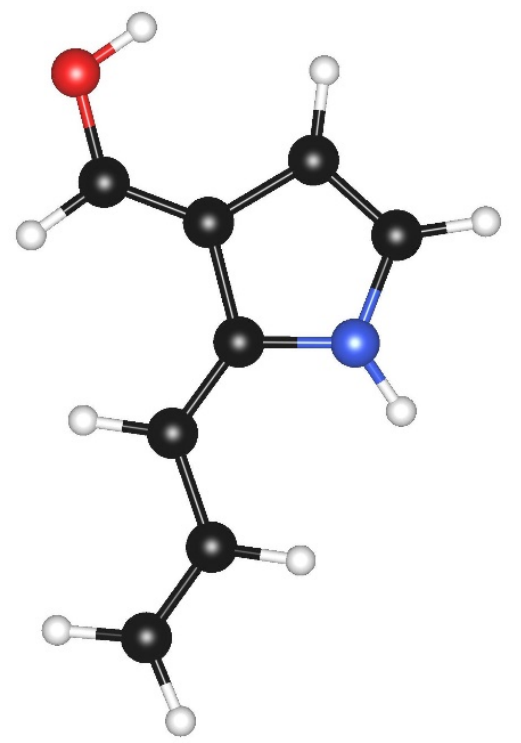

HF $(M 062 X / 6-31+G(d, p))=-440.0059954$ Hartrees

Imaginary Frequencies: none found

Zero-point correction $=0.152594$ (Hartree/Particle)

Thermal correction $=0.117225$ Hartrees

Coordinates from last standard orientation:

\begin{tabular}{|c|c|c|c|c|c|}
\hline \multirow{2}{*}{$\begin{array}{l}\text { Center } \\
\text { Number }\end{array}$} & \multirow{2}{*}{$\begin{array}{l}\text { Atomic } \\
\text { Number }\end{array}$} & \multirow{2}{*}{$\begin{array}{c}\text { Atomic } \\
\text { Type }\end{array}$} & \multicolumn{3}{|c|}{ Coordinates (Angstroms) } \\
\hline & & & $x$ & Y & Z \\
\hline & & & & 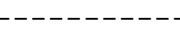 & ---- \\
\hline 1 & 6 & 0 & -0.644083 & 0.509298 & -0.000447 \\
\hline 2 & 6 & 0 & -1.887949 & -0.224226 & -0.000632 \\
\hline 3 & 6 & 0 & -3.079773 & 0.391077 & 0.000796 \\
\hline 4 & 1 & 0 & -3.166834 & 1.476558 & 0.002498 \\
\hline 5 & 1 & 0 & -1.872166 & -1.311002 & -0.002011 \\
\hline 6 & 1 & 0 & -0.715933 & 1.596332 & -0.000073 \\
\hline 7 & 6 & 0 & 0.588202 & -0.052594 & -0.000392 \\
\hline 8 & 6 & 0 & 1.872976 & 0.702316 & -0.000040 \\
\hline 9 & 6 & 0 & 0.954038 & -1.468290 & -0.000246 \\
\hline 10 & 6 & 0 & 2.302438 & -1.542486 & 0.000444 \\
\hline 11 & 1 & 0 & 0.276967 & -2.308476 & -0.000384 \\
\hline 12 & 1 & 0 & 2.936660 & -2.418586 & 0.000876 \\
\hline 13 & 6 & 0 & 2.093801 & 2.028942 & -0.000369 \\
\hline 14 & 1 & 0 & 3.098982 & 2.434458 & -0.000048 \\
\hline 15 & 1 & 0 & 1.267319 & 2.727208 & -0.001050 \\
\hline 16 & 8 & 0 & -4.244667 & -0.313004 & -0.000929 \\
\hline 17 & 1 & 0 & -4.998621 & 0.283447 & 0.007505 \\
\hline 18 & 7 & 0 & 2.868435 & -0.279594 & 0.000626 \\
\hline 19 & 1 & 0 & 3.854014 & -0.082973 & 0.001054 \\
\hline
\end{tabular}

\section{Enol C7}




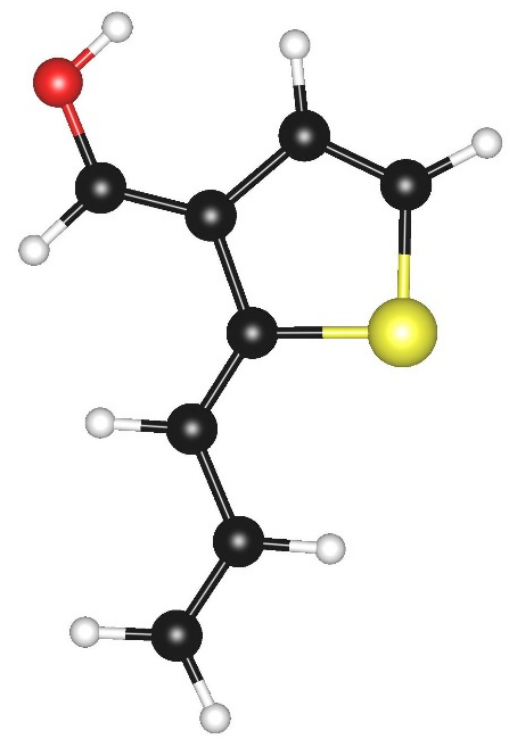

HF $(M 062 X / 6-31+G(d, p))=-782.8262469$ Hartrees

Imaginary Frequencies: none found

Zero-point correction $=0.137839$ (Hartree/Particle)

Thermal correction $=0.101024$ Hartrees

Coordinates from last standard orientation:

\begin{tabular}{|c|c|c|c|c|c|}
\hline \multirow{2}{*}{$\begin{array}{l}\text { Center } \\
\text { Number }\end{array}$} & \multirow{2}{*}{$\begin{array}{l}\text { Atomic } \\
\text { Number }\end{array}$} & \multirow{2}{*}{$\begin{array}{c}\text { Atomic } \\
\text { Type }\end{array}$} & \multicolumn{3}{|c|}{ Coordinates (Angstroms) } \\
\hline & & & $\mathrm{x}$ & $\mathrm{Y}$ & Z \\
\hline 1 & 6 & 0 & -0.986210 & 0.518949 & -0.000517 \\
\hline 2 & 6 & 0 & -2.208741 & -0.249442 & -0.001069 \\
\hline 3 & 6 & 0 & -3.412937 & 0.342563 & 0.001037 \\
\hline 4 & 1 & 0 & -3.521740 & 1.426144 & 0.003651 \\
\hline 5 & 1 & 0 & -2.174655 & -1.335101 & -0.003191 \\
\hline 6 & 1 & 0 & -1.101911 & 1.602027 & 0.000192 \\
\hline 7 & 6 & 0 & 0.269851 & 0.009621 & -0.000595 \\
\hline 8 & 6 & 0 & 1.497249 & 0.848254 & -0.000215 \\
\hline 9 & 6 & 0 & 0.623468 & -1.407149 & -0.000604 \\
\hline 10 & 6 & 0 & 1.945237 & -1.640982 & 0.000265 \\
\hline 11 & 1 & 0 & -0.111655 & -2.202411 & -0.000881 \\
\hline 12 & 1 & 0 & 2.431071 & -2.608284 & 0.000724 \\
\hline 13 & 6 & 0 & 1.594223 & 2.184578 & -0.000719 \\
\hline 14 & 1 & 0 & 2.555691 & 2.684925 & -0.000362 \\
\hline 15 & 1 & 0 & 0.708368 & 2.810237 & -0.001564 \\
\hline 16 & 8 & 0 & -4.559402 & -0.384678 & -0.000762 \\
\hline 17 & 1 & 0 & -5.328577 & 0.192524 & 0.008869 \\
\hline 18 & 16 & 0 & 2.942862 & -0.195688 & 0.000822 \\
\hline
\end{tabular}

\section{Aldehyde C8}




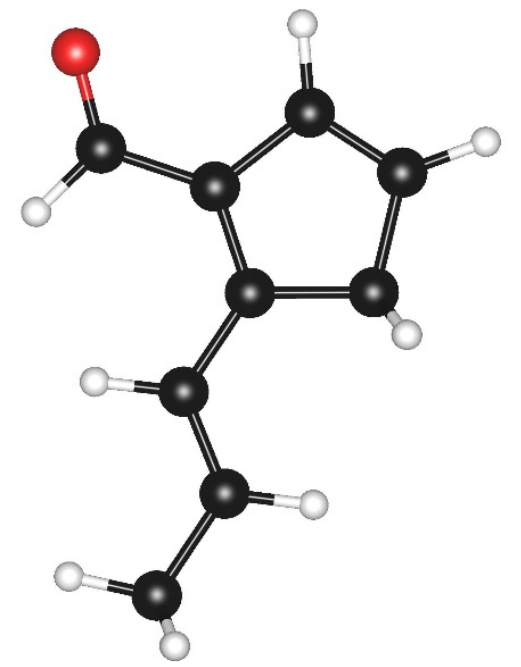

HF $(M 062 X / 6-31+G(d, p))=-423.9812446$ Hartrees

Imaginary Frequencies: none found

Zero-point correction $=0.164541$ (Hartree/Particle)

Thermal correction $=0.128672$ Hartrees

Coordinates from last standard orientation:

\begin{tabular}{|c|c|c|c|c|c|}
\hline \multirow{2}{*}{$\begin{array}{l}\text { Center } \\
\text { Number }\end{array}$} & \multirow{2}{*}{$\begin{array}{l}\text { Atomic } \\
\text { Number }\end{array}$} & \multirow{2}{*}{$\begin{array}{c}\text { Atomic } \\
\text { Type }\end{array}$} & \multicolumn{3}{|c|}{ Coordinates (Angstroms) } \\
\hline & & & $\mathrm{x}$ & Y & $\mathrm{z}$ \\
\hline 1 & 6 & 0 & -0.809016 & 0.423497 & -0.002025 \\
\hline 2 & 6 & 0 & -1.935255 & -0.313468 & 0.000231 \\
\hline 3 & 6 & 0 & -3.246646 & 0.344971 & -0.000648 \\
\hline 4 & 1 & 0 & -3.215960 & 1.456065 & -0.003874 \\
\hline 5 & 1 & 0 & -1.932108 & -1.399933 & 0.003048 \\
\hline 6 & 1 & 0 & -0.917563 & 1.508658 & -0.004529 \\
\hline 7 & 6 & 0 & 0.546942 & -0.095455 & -0.001192 \\
\hline 8 & 6 & 0 & 1.689378 & 0.644532 & -0.000237 \\
\hline 9 & 6 & 0 & 0.908044 & -1.528387 & -0.000880 \\
\hline 10 & 6 & 0 & 2.244217 & -1.654121 & 0.000339 \\
\hline 11 & 1 & 0 & 0.191576 & -2.341389 & -0.001835 \\
\hline 12 & 1 & 0 & 2.806685 & -2.579291 & 0.000563 \\
\hline 13 & 6 & 0 & 1.858931 & 2.128941 & 0.000963 \\
\hline 14 & 1 & 0 & 2.433405 & 2.452467 & -0.874265 \\
\hline 15 & 1 & 0 & 0.905118 & 2.659341 & -0.008717 \\
\hline 16 & 6 & 0 & 2.869788 & -0.288211 & 0.000843 \\
\hline 17 & 1 & 0 & 3.507257 & -0.122816 & 0.880871 \\
\hline 18 & 1 & 0 & 3.508437 & -0.122969 & -0.878363 \\
\hline 19 & 1 & 0 & 2.415857 & 2.453168 & 0.887303 \\
\hline 20 & 8 & 0 & -4.307625 & -0.242137 & 0.001929 \\
\hline
\end{tabular}




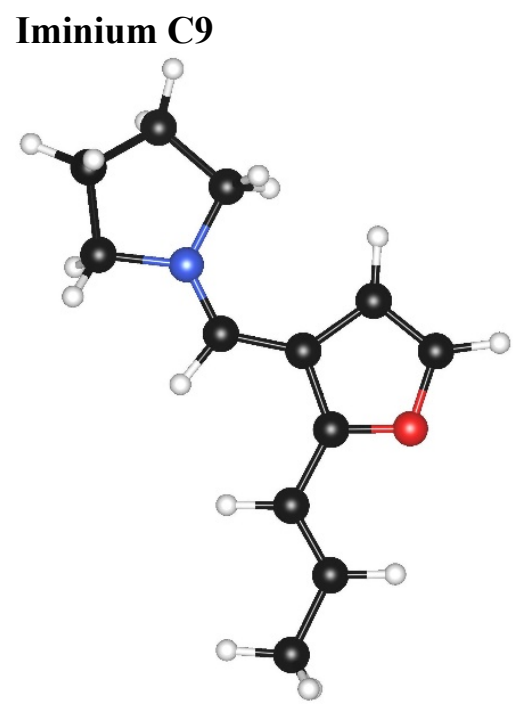

HF $(M 062 X / 6-31+G(d, p))=-596.4039316$ Hartrees

Imaginary Frequencies: none found

Zero-point correction $=0.262326$ (Hartree/Particle $)$

Thermal correction $=0.221163$ Hartrees

Coordinates from last standard orientation:

\begin{tabular}{|c|c|c|c|c|c|}
\hline \multirow{2}{*}{$\begin{array}{l}\text { Center } \\
\text { Number }\end{array}$} & \multirow{2}{*}{$\begin{array}{l}\text { Atomic } \\
\text { Number }\end{array}$} & \multirow{2}{*}{$\begin{array}{c}\text { Atomic } \\
\text { Type }\end{array}$} & \multicolumn{3}{|c|}{ Coordinates (Angstroms) } \\
\hline & & & $\mathrm{X}$ & $\mathrm{Y}$ & $\mathrm{z}$ \\
\hline 1 & 6 & 0 & -1.029896 & -0.553017 & 0.031467 \\
\hline 2 & 6 & 0 & 0.200158 & 0.049768 & 0.040004 \\
\hline 3 & 6 & 0 & 1.361853 & -0.757862 & 0.068274 \\
\hline 4 & 1 & 0 & 1.245447 & -1.841659 & 0.081058 \\
\hline 5 & 7 & 0 & 2.588481 & -0.321630 & 0.075936 \\
\hline 6 & 6 & 0 & 3.782286 & -1.200811 & 0.081844 \\
\hline 7 & 1 & 0 & 3.601432 & -2.072076 & -0.550068 \\
\hline 8 & 1 & 0 & 3.966629 & -1.528914 & 1.110752 \\
\hline 9 & 6 & 0 & 4.888849 & -0.273542 & -0.412557 \\
\hline 10 & 1 & 0 & 4.872621 & -0.217486 & -1.505503 \\
\hline 11 & 1 & 0 & 5.876197 & -0.615050 & -0.099745 \\
\hline 12 & 6 & 0 & 4.496086 & 1.074416 & 0.203239 \\
\hline 13 & 1 & 0 & 4.971657 & 1.924742 & -0.286613 \\
\hline 14 & 1 & 0 & 4.763693 & 1.098937 & 1.263845 \\
\hline 15 & 6 & 0 & 2.973928 & 1.108129 & 0.046074 \\
\hline 16 & 1 & 0 & 2.461222 & 1.644850 & 0.847905 \\
\hline 17 & 1 & 0 & 2.666812 & 1.525597 & -0.919345 \\
\hline 18 & 1 & 0 & 0.290263 & 1.130622 & 0.020182 \\
\hline 19 & 1 & 0 & -1.061948 & -1.642572 & 0.051452 \\
\hline 20 & 6 & 0 & -2.281402 & 0.117197 & -0.000771 \\
\hline 21 & 6 & 0 & -3.516982 & -0.517815 & -0.007806 \\
\hline 22 & 6 & 0 & -2.564809 & 1.536313 & -0.033555 \\
\hline 23 & 6 & 0 & -3.907637 & 1.643132 & -0.056993 \\
\hline 24 & 1 & 0 & -1.861789 & 2.355477 & -0.039180 \\
\hline 25 & 1 & 0 & -4.593638 & 2.474543 & -0.085026 \\
\hline 26 & 6 & 0 & -3.944101 & -1.937853 & 0.015124 \\
\hline 27 & 1 & 0 & -4.539039 & -2.164906 & -0.873957 \\
\hline 28 & 1 & 0 & -3.086170 & -2.609543 & 0.042844 \\
\hline 29 & 1 & 0 & -4.567148 & -2.127484 & 0.893767 \\
\hline
\end{tabular}




\section{Iminium C10}

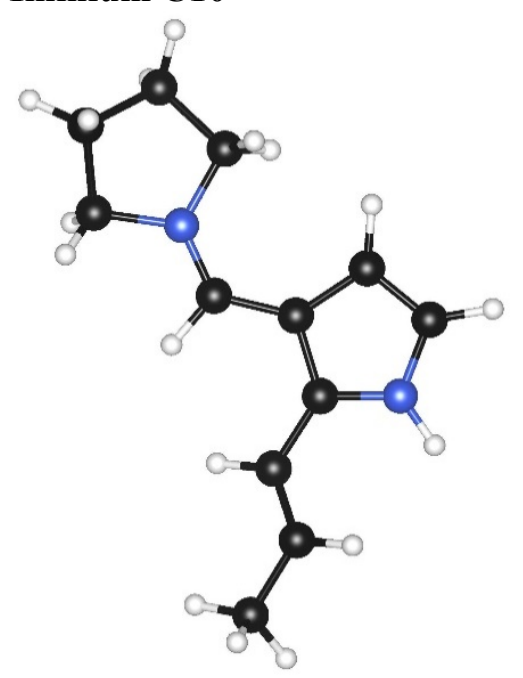

HF $(M 062 X / 6-31+G(d, p))=-576.5659099$ Hartrees

Imaginary Frequencies: none found

Zero-point correction $=0.275330$ (Hartree/Particle)

Thermal correction $=0.233933$ Hartrees

Coordinates from last standard orientation:

\begin{tabular}{|c|c|c|c|c|c|}
\hline \multirow{2}{*}{$\begin{array}{l}\text { Center } \\
\text { Number }\end{array}$} & \multirow{2}{*}{$\begin{array}{l}\text { Atomic } \\
\text { Number }\end{array}$} & \multirow{2}{*}{$\begin{array}{c}\text { Atomic } \\
\text { Type }\end{array}$} & \multicolumn{3}{|c|}{ Coordinates (Angstroms) } \\
\hline & & & $\mathrm{x}$ & Y & z \\
\hline 1 & 6 & 0 & -1.035455 & -0.535357 & 0.009432 \\
\hline 2 & 6 & 0 & 0.203500 & 0.064205 & 0.032468 \\
\hline 3 & 6 & 0 & 1.356139 & -0.744369 & 0.049630 \\
\hline 4 & 1 & 0 & 1.235759 & -1.827775 & 0.042462 \\
\hline 5 & 7 & 0 & 2.590024 & -0.315720 & 0.069404 \\
\hline 6 & 6 & 0 & 3.776238 & -1.201227 & 0.062986 \\
\hline 7 & 1 & 0 & 3.595471 & -2.057404 & -0.589616 \\
\hline 8 & 1 & 0 & 3.957019 & -1.555251 & 1.084113 \\
\hline 9 & 6 & 0 & 4.891058 & -0.271163 & -0.408106 \\
\hline 10 & 1 & 0 & 4.880549 & -0.195323 & -1.499969 \\
\hline 11 & 1 & 0 & 5.875369 & -0.622668 & -0.096520 \\
\hline 12 & 6 & 0 & 4.501447 & 1.068234 & 0.228361 \\
\hline 13 & 1 & 0 & 4.983188 & 1.924532 & -0.244980 \\
\hline 14 & 1 & 0 & 4.765011 & 1.073310 & 1.290326 \\
\hline 15 & 6 & 0 & 2.979804 & 1.110337 & 0.066258 \\
\hline 16 & 1 & 0 & 2.467651 & 1.636032 & 0.876139 \\
\hline 17 & 1 & 0 & 2.677616 & 1.549605 & -0.891395 \\
\hline 18 & 1 & 0 & 0.295975 & 1.144962 & 0.034850 \\
\hline 19 & 1 & 0 & -1.062949 & -1.626020 & 0.014032 \\
\hline 20 & 6 & 0 & -2.282589 & 0.125274 & -0.016693 \\
\hline 21 & 6 & 0 & -3.525787 & -0.539540 & -0.012641 \\
\hline 22 & 6 & 0 & -2.558653 & 1.539827 & -0.046432 \\
\hline 23 & 6 & 0 & -3.910376 & 1.683390 & -0.058770 \\
\hline 24 & 1 & 0 & -1.840095 & 2.345704 & -0.065101 \\
\hline
\end{tabular}




$\begin{array}{rrrrrr}25 & 1 & 0 & -4.532599 & 2.564445 & -0.085685 \\ 26 & 6 & 0 & -3.860400 & -1.992444 & 0.051421 \\ 27 & 1 & 0 & -4.825478 & -2.189400 & -0.421248 \\ 28 & 1 & 0 & -3.110914 & -2.591754 & -0.468367 \\ 29 & 1 & 0 & -3.914111 & -2.336304 & 1.089197 \\ 30 & 7 & 0 & -4.474176 & 0.414444 & -0.037273 \\ 31 & 1 & 0 & -5.467959 & 0.229233 & -0.040633 \\ -\end{array}$

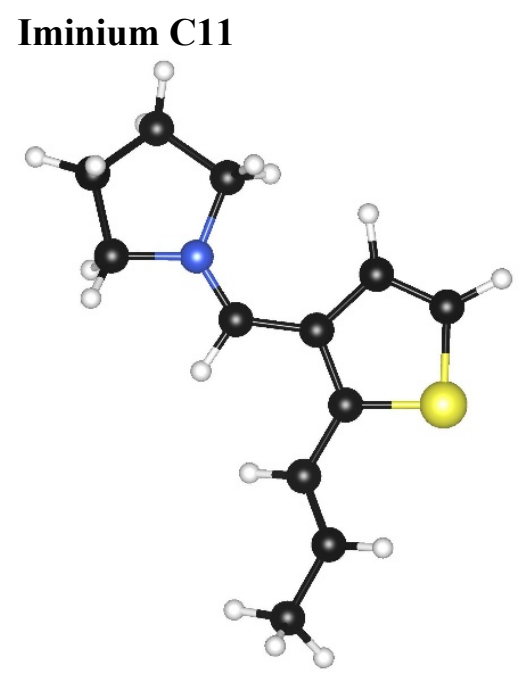

HF $(M 062 X / 6-31+G(d, p))=-919.3693188$ Hartrees

Imaginary Frequencies: none found

Zero-point correction $=0.258819$ (Hartree/Particle)

Thermal correction $=0.216855$ Hartrees

\section{Coordinates from last standard orientation:}

\begin{tabular}{|c|c|c|c|c|c|}
\hline \multirow{2}{*}{$\begin{array}{l}\text { Center } \\
\text { Number }\end{array}$} & \multirow{2}{*}{$\begin{array}{l}\text { Atomic } \\
\text { Number }\end{array}$} & \multirow{2}{*}{$\begin{array}{c}\text { Atomic } \\
\text { Type }\end{array}$} & \multicolumn{3}{|c|}{ Coordinates (Angstroms) } \\
\hline & & & $\mathrm{X}$ & Y & Z \\
\hline & & & -1 & -- & --- \\
\hline 1 & 6 & 0 & 0.681402 & 0.586010 & 0.030573 \\
\hline 2 & 6 & 0 & -0.538678 & -0.037826 & 0.041785 \\
\hline 3 & 6 & 0 & -1.711651 & 0.753549 & 0.065270 \\
\hline 4 & 1 & 0 & -1.610285 & 1.838874 & 0.072778 \\
\hline 5 & 7 & 0 & -2.932478 & 0.300491 & 0.074203 \\
\hline 6 & 6 & 0 & -4.137822 & 1.163495 & 0.075021 \\
\hline 7 & 1 & 0 & -3.967927 & 2.034468 & -0.560378 \\
\hline 8 & 1 & 0 & -4.328005 & 1.493561 & 1.102262 \\
\hline 9 & 6 & 0 & -5.231274 & 0.219494 & -0.416883 \\
\hline 10 & 1 & 0 & -5.212598 & 0.158847 & -1.509553 \\
\hline 11 & 1 & 0 & -6.223598 & 0.549178 & -0.107116 \\
\hline 12 & 6 & 0 & -4.821473 & -1.120332 & 0.205438 \\
\hline 13 & 1 & 0 & -5.284942 & -1.979206 & -0.281133 \\
\hline 14 & 1 & 0 & -5.090234 & -1.143583 & 1.265786 \\
\hline 15 & 6 & 0 & -3.298718 & -1.134344 & 0.050627 \\
\hline 16 & 1 & 0 & -2.780293 & -1.660532 & 0.855769 \\
\hline 17 & 1 & 0 & -2.984543 & -1.552505 & -0.912219 \\
\hline 18 & 1 & 0 & -0.616921 & -1.119465 & 0.027975 \\
\hline 19 & 1 & 0 & 0.686535 & 1.676649 & 0.045461 \\
\hline 20 & 6 & 0 & 1.958413 & -0.046933 & 0.002381 \\
\hline
\end{tabular}




$\begin{array}{rrrrrr}21 & 6 & 0 & 3.141810 & 0.694508 & -0.001821 \\ 22 & 6 & 0 & 2.202870 & -1.467387 & -0.024019 \\ 23 & 6 & 0 & 3.524684 & -1.764229 & -0.046067 \\ 24 & 1 & 0 & 1.428233 & -2.224437 & -0.027574 \\ 25 & 1 & 0 & 3.994034 & -2.737842 & -0.070103 \\ 26 & 6 & 0 & 3.304046 & 2.184892 & 0.026275 \\ 27 & 1 & 0 & 4.357267 & 2.466097 & -0.017915 \\ 28 & 1 & 0 & 2.801429 & 2.649040 & -0.827348 \\ 29 & 1 & 0 & 2.887597 & 2.604384 & 0.947097 \\ 30 & 16 & 0 & 4.501872 & -0.333897 & -0.035918 \\ - & & 0 & & \end{array}$

\section{Trienamine C12}

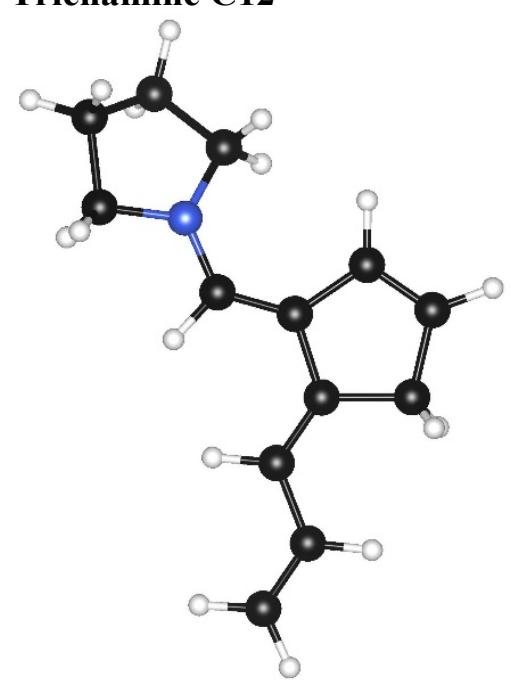

HF $(M 062 X / 6-31+G(d, p))=-560.0783088$ Hartrees

Imaginary Frequencies: none found

Zero-point correction $=0.271150$ (Hartree/Particle)

Thermal correction $=0.228525$ Hartrees

Coordinates from last standard orientation:

\begin{tabular}{|c|c|c|c|c|c|}
\hline \multirow{2}{*}{$\begin{array}{l}\text { Center } \\
\text { Number }\end{array}$} & \multirow{2}{*}{$\begin{array}{l}\text { Atomic } \\
\text { Number }\end{array}$} & \multirow{2}{*}{$\begin{array}{c}\text { Atomic } \\
\text { Type }\end{array}$} & \multicolumn{3}{|c|}{ Coordinates (Angstroms) } \\
\hline & & & $\mathrm{x}$ & Y & Z \\
\hline 1 & 6 & 0 & -1.109614 & 0.654965 & -0.030147 \\
\hline 2 & 6 & 0 & 0.194354 & 0.042078 & -0.036391 \\
\hline 3 & 6 & 0 & 1.329504 & 0.791170 & -0.058162 \\
\hline 4 & 1 & 0 & 1.251764 & 1.877890 & -0.070681 \\
\hline 5 & 7 & 0 & 2.603294 & 0.322090 & -0.059668 \\
\hline 6 & 6 & 0 & 3.787258 & 1.171440 & -0.066801 \\
\hline 7 & 1 & 0 & 3.655207 & 2.024276 & 0.607247 \\
\hline 8 & 1 & 0 & 3.994432 & 1.559157 & -1.076091 \\
\hline 9 & 6 & 0 & 4.891084 & 0.215756 & 0.389739 \\
\hline 10 & 1 & 0 & 4.895083 & 0.153180 & 1.483190 \\
\hline 11 & 1 & 0 & 5.883446 & 0.528765 & 0.057948 \\
\hline 12 & 6 & 0 & 4.445490 & -1.123153 & -0.212183 \\
\hline 13 & 1 & 0 & 4.904156 & -1.987770 & 0.272022 \\
\hline 14 & 1 & 0 & 4.700688 & -1.154890 & -1.276804 \\
\hline 15 & 6 & 0 & 2.921539 & -1.096137 & -0.045336 \\
\hline
\end{tabular}




$\begin{array}{rrrrrr}16 & 1 & 0 & 2.399553 & -1.619072 & -0.857048 \\ 17 & 1 & 0 & 2.599093 & -1.548608 & 0.905239 \\ 18 & 1 & 0 & 0.265368 & -1.041768 & -0.019968 \\ 19 & 1 & 0 & -1.124153 & 1.745695 & -0.049179 \\ 20 & 6 & 0 & -2.302215 & 0.013080 & -0.002919 \\ 21 & 6 & 0 & -3.642997 & 0.649699 & 0.003076 \\ 22 & 6 & 0 & -2.532552 & -1.436011 & 0.030090 \\ 23 & 6 & 0 & -3.843412 & -1.720898 & 0.056732 \\ 24 & 1 & 0 & -1.738083 & -2.174152 & 0.033627 \\ 25 & 1 & 0 & -4.268828 & -2.717858 & 0.085384 \\ 26 & 6 & 0 & -3.933289 & 1.955001 & -0.024477 \\ 27 & 1 & 0 & -4.962576 & 2.300515 & -0.016659 \\ 28 & 1 & 0 & -3.158830 & 2.715574 & -0.056914 \\ 29 & 6 & 0 & -5.347050 & -0.427585 & -0.826149 \\ 30 & 1 & 0 & -5.316884 & -0.391048 & 0.935432 \\ 31 & 1 & 0 & 0--104625\end{array}$

\section{Fulvene trienamine C12'}

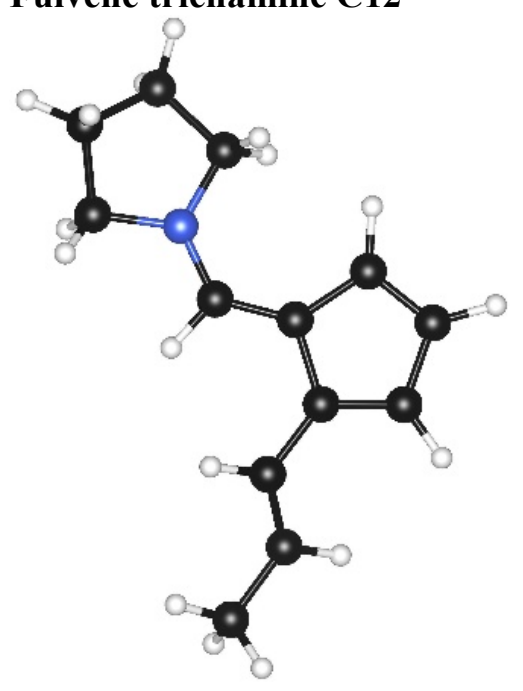

HF $(M 062 X / 6-31+G(d, p))=-560.0894486$ Hartrees Imaginary Frequencies: none found Zero-point correction $=0.272533$ (Hartree/Particle) Thermal correction $=0.231392$ Hartrees

Coordinates from last standard orientation:

\begin{tabular}{|c|c|c|c|c|c|}
\hline \multirow{2}{*}{$\begin{array}{l}\text { Center } \\
\text { Number }\end{array}$} & \multirow{2}{*}{$\begin{array}{l}\text { Atomic } \\
\text { Number }\end{array}$} & \multirow{2}{*}{$\begin{array}{c}\text { Atomic } \\
\text { Type }\end{array}$} & \multicolumn{3}{|c|}{ Coordinates (Angstroms) } \\
\hline & & & $\mathrm{x}$ & Y & $\mathrm{z}$ \\
\hline--- & & & & -1 & ------- \\
\hline 1 & 6 & 0 & -1.795494 & 0.560098 & -0.147086 \\
\hline 2 & 6 & 0 & -0.336870 & 0.705420 & -0.090116 \\
\hline 3 & 6 & 0 & 0.494497 & -0.388127 & -0.079518 \\
\hline 4 & 1 & 0 & 0.028880 & -1.370471 & -0.145669 \\
\hline 5 & 7 & 0 & 1.831237 & -0.447230 & 0.002785 \\
\hline 6 & 6 & 0 & 2.572624 & -1.711030 & -0.021933 \\
\hline 7 & 1 & 0 & 2.155421 & -2.383212 & -0.776822 \\
\hline 8 & 1 & 0 & 2.517439 & -2.207966 & 0.956886 \\
\hline 9 & 6 & 0 & 4.001041 & -1.264448 & -0.329892 \\
\hline
\end{tabular}




$\begin{array}{rrrrrr}10 & 1 & 0 & 4.120027 & -1.121033 & -1.409116 \\ 11 & 1 & 0 & 4.747053 & -1.986876 & 0.007461 \\ 12 & 6 & 0 & 4.090551 & 0.082827 & 0.396651 \\ 13 & 1 & 0 & 4.900797 & 0.717215 & 0.031999 \\ 14 & 1 & 0 & 4.244982 & -0.082206 & 1.468207 \\ 15 & 6 & 0 & 2.711514 & 0.707325 & 0.159907 \\ 16 & 1 & 0 & 2.374384 & 1.325978 & 0.998009 \\ 17 & 1 & 0 & 2.684937 & 1.320947 & -0.750605 \\ 18 & 6 & 0 & -2.525856 & -0.706416 & -0.224974 \\ 19 & 6 & 0 & -3.749342 & -0.920514 & 0.277028 \\ 20 & 6 & 0 & -4.516144 & -2.200881 & 0.133019 \\ 21 & 1 & 0 & -5.457848 & -2.039910 & -0.404197 \\ 22 & 1 & 0 & -3.936860 & -2.948249 & -0.417085 \\ 23 & 1 & 0 & -2.043837 & -1.534220 & -0.749745 \\ 24 & 1 & 0 & -4.232961 & -0.115335 & 0.830282 \\ 25 & 6 & 0 & -1.263345 & 2.788070 & -0.036384 \\ 26 & 1 & 0 & -1.395608 & 3.862998 & -0.010343 \\ 27 & 6 & 0 & -0.062468 & 2.133082 & -0.021649 \\ 28 & 1 & 0 & 0.907381 & 2.606429 & 0.016307 \\ 29 & 6 & 0 & -2.332084 & 1.822768 & -0.115045 \\ 30 & 1 & 0 & -3.388119 & 2.057488 & -0.176693 \\ 31 & 1 & 0 & -4.776464 & -2.620009 & 1.111572 \\ ------------------------------------------------\end{array}$

\section{Trienamine C13}

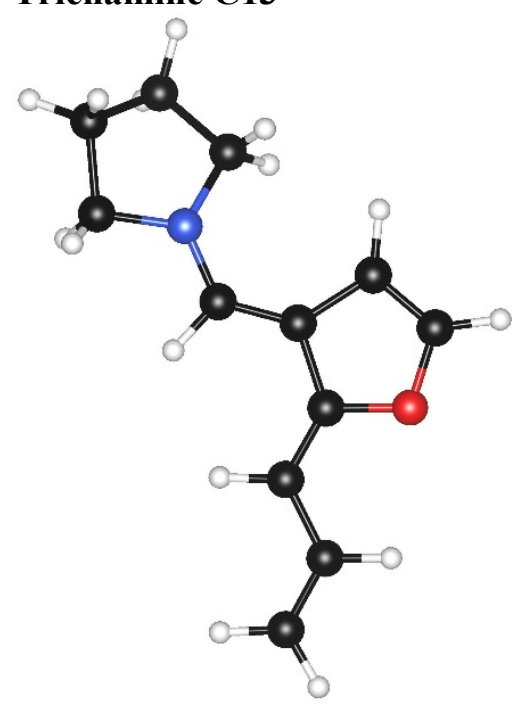

HF $(M 062 X / 6-31+G(d, p))=-595.9798717$ Hartrees

Imaginary Frequencies: none found

Zero-point correction $=0.247648$ (Hartree/Particle)

Thermal correction $=0.206087$ Hartrees

\section{Coordinates from last standard orientation:}

\begin{tabular}{cccccc} 
Center & Atomic & Atomic & \multicolumn{2}{c}{ Coordinates } & \multicolumn{2}{c}{ (Angstroms) } \\
Number & Number & Type & $X$ & Y & $Z$ \\
-1 & 6 & 0 & -1.115178 & -0.643961 & 0.028256 \\
2 & 6 & 0 & 0.187212 & -0.037183 & 0.034621 \\
3 & 6 & 0 & 1.321319 & -0.790558 & 0.063178
\end{tabular}




$\begin{array}{rrrrrr}4 & 1 & 0 & 1.239313 & -1.876818 & 0.082184 \\ 5 & 7 & 0 & 2.594317 & -0.325973 & 0.064005 \\ 6 & 6 & 0 & 3.776473 & -1.179173 & 0.080529 \\ 7 & 1 & 0 & 3.640690 & -2.039397 & -0.583022 \\ 8 & 1 & 0 & 3.981581 & -1.554622 & 1.094580 \\ 9 & 6 & 0 & 4.882784 & -0.232015 & -0.386978 \\ 10 & 1 & 0 & 4.886219 & -0.180902 & -1.480990 \\ 11 & 1 & 0 & 5.874183 & -0.545041 & -0.052602 \\ 12 & 6 & 0 & 4.442345 & 1.114474 & 0.201753 \\ 13 & 1 & 0 & 4.903504 & 1.972370 & -0.291757 \\ 14 & 1 & 0 & 4.698564 & 1.156376 & 1.265731 \\ 15 & 6 & 0 & 2.918245 & 1.091612 & 0.036278 \\ 16 & 1 & 0 & 2.398502 & 1.623554 & 0.843330 \\ 17 & 1 & 0 & 2.596626 & 1.534926 & -0.918625 \\ 18 & 1 & 0 & 0.258404 & 1.046751 & 0.012853 \\ 19 & 1 & 0 & -1.144815 & -1.733673 & 0.048872 \\ 20 & 6 & 0 & -2.299560 & 0.016325 & 0.000168 \\ 21 & 6 & 0 & -3.639086 & -0.601527 & -0.005936 \\ 22 & 6 & 0 & -2.559533 & 1.453420 & -0.030874 \\ 23 & 8 & 0 & -4.575476 & 0.423566 & -0.037251 \\ 24 & 6 & 0 & -3.890727 & 1.608757 & -0.051081 \\ 25 & 1 & 0 & -1.829265 & 2.248655 & -0.037067 \\ 26 & 1 & 0 & -4.510986 & 2.493022 & -0.075059 \\ 27 & 6 & 0 & -4.045218 & -1.875883 & 0.013156 \\ 28 & 1 & 0 & -5.100450 & -2.116394 & 0.004326 \\ 29 & 1 & 0 & -3.322950 & -2.681251 & 0.038809 \\ -------------------------1\end{array}$

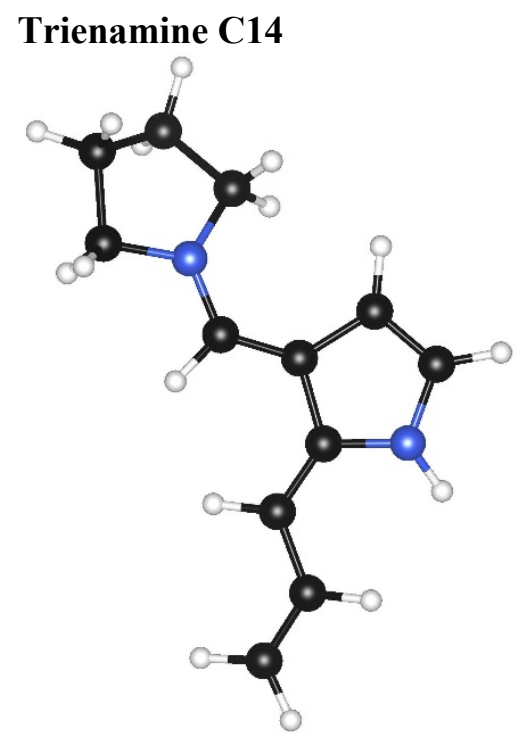

HF $(M 062 X / 6-31+G(d, p))=-576.1231782$ Hartrees

Imaginary Frequencies: none found

Zero-point correction $=0.259749$ (Hartree/Particle)

Thermal correction $=0.217801$ Hartrees

Coordinates from last standard orientation:

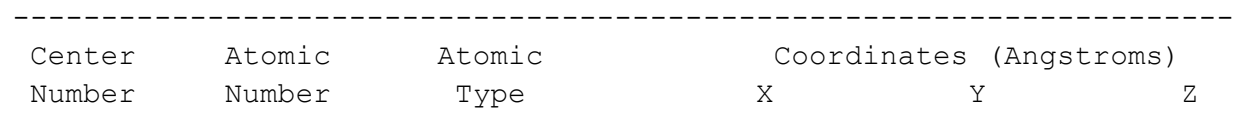




\begin{tabular}{|c|c|c|c|c|c|}
\hline 1 & 6 & 0 & -1.117622 & 0.645405 & -0.014131 \\
\hline 2 & 6 & 0 & 0.186211 & 0.040611 & -0.032851 \\
\hline 3 & 6 & 0 & 1.320604 & 0.792825 & -0.023717 \\
\hline 4 & 1 & 0 & 1.240504 & 1.879120 & 0.000643 \\
\hline 5 & 7 & 0 & 2.595041 & 0.326423 & -0.035061 \\
\hline 6 & 6 & 0 & 3.776555 & 1.178947 & -0.025208 \\
\hline 7 & 1 & 0 & 3.648554 & 2.008122 & 0.678447 \\
\hline 8 & 1 & 0 & 3.972607 & 1.600951 & -1.022952 \\
\hline 9 & 6 & 0 & 4.887082 & 0.211623 & 0.388023 \\
\hline 10 & 1 & 0 & 4.901311 & 0.111271 & 1.478547 \\
\hline 11 & 1 & 0 & 5.875411 & 0.539098 & 0.058175 \\
\hline 12 & 6 & 0 & 4.439896 & -1.106993 & -0.256025 \\
\hline 13 & 1 & 0 & 4.904473 & -1.986518 & 0.194616 \\
\hline 14 & 1 & 0 & 4.686855 & -1.101495 & -1.323034 \\
\hline 15 & 6 & 0 & 2.917233 & -1.090523 & -0.076233 \\
\hline 16 & 1 & 0 & 2.390169 & -1.584273 & -0.902901 \\
\hline 17 & 1 & 0 & 2.603359 & -1.579228 & 0.859031 \\
\hline 18 & 1 & 0 & 0.257517 & -1.043383 & -0.053094 \\
\hline 19 & 1 & 0 & -1.147533 & 1.735186 & 0.003080 \\
\hline 20 & 6 & 0 & -2.301567 & -0.018587 & -0.013628 \\
\hline 21 & 6 & 0 & -3.642504 & 0.619790 & 0.002428 \\
\hline 22 & 6 & 0 & -2.542703 & -1.460490 & 0.004420 \\
\hline 23 & 6 & 0 & -3.876536 & -1.653958 & 0.074055 \\
\hline 24 & 1 & 0 & -1.792646 & -2.237183 & -0.001203 \\
\hline 25 & 1 & 0 & -4.432069 & -2.581096 & 0.116981 \\
\hline 26 & 6 & 0 & -3.985861 & 1.917739 & -0.101508 \\
\hline 27 & 1 & 0 & -5.022070 & 2.233512 & -0.061308 \\
\hline 28 & 1 & 0 & -3.229142 & 2.680534 & -0.229680 \\
\hline 29 & 7 & 0 & -4.551134 & -0.440159 & 0.138617 \\
\hline 30 & 1 & 0 & -5.529379 & -0.336790 & -0.073992 \\
\hline
\end{tabular}

\section{Fulvene trienamine C14'}

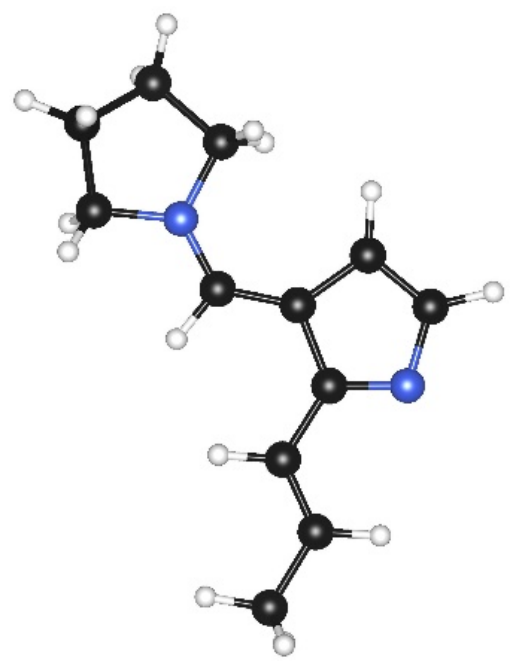

HF $(M 062 X / 6-31+G(d, p))=-576.1377698$ Hartrees Imaginary Frequencies: none found Zero-point correction $=0.261207$ (Hartree/Particle)

Thermal correction $=0.220211$ Hartrees 
Coordinates from last standard orientation:

\begin{tabular}{|c|c|c|c|c|c|}
\hline \multirow{2}{*}{$\begin{array}{l}\text { Center } \\
\text { Number }\end{array}$} & \multirow{2}{*}{$\begin{array}{l}\text { Atomic } \\
\text { Number }\end{array}$} & \multirow{2}{*}{$\begin{array}{c}\text { Atomic } \\
\text { Type }\end{array}$} & \multicolumn{3}{|c|}{ Coordinates (Angstroms) } \\
\hline & & & $\mathrm{x}$ & Y & Z \\
\hline 1 & 6 & 0 & -1.768991 & 0.520421 & -0022940 \\
\hline 2 & 6 & 0 & -0.312468 & 0.653692 & -0.025411 \\
\hline 3 & 6 & 0 & 0.532663 & -0.432109 & -0.011580 \\
\hline 4 & 1 & 0 & 0.086054 & -1.424761 & -0.029224 \\
\hline 5 & 7 & 0 & 1.867430 & -0.456605 & 0.018610 \\
\hline 6 & 6 & 0 & 2.645620 & -1.700682 & 0.006883 \\
\hline 7 & 1 & 0 & 2.226152 & -2.404954 & -0.716374 \\
\hline 8 & 1 & 0 & 2.630738 & -2.167335 & 1.001336 \\
\hline 9 & 6 & 0 & 4.051644 & -1.222716 & -0.354197 \\
\hline 10 & 1 & 0 & 4.138716 & -1.111996 & -1.440255 \\
\hline 11 & 1 & 0 & 4.826428 & -1.912626 & -0.014180 \\
\hline 12 & 6 & 0 & 4.120830 & 0.149819 & 0.325581 \\
\hline 13 & 1 & 0 & 4.904973 & 0.792812 & -0.078411 \\
\hline 14 & 1 & 0 & 4.301885 & 0.025305 & 1.398391 \\
\hline 15 & 6 & 0 & 2.721564 & 0.728631 & 0.097859 \\
\hline 16 & 1 & 0 & 2.386383 & 1.376494 & 0.913449 \\
\hline 17 & 1 & 0 & 2.655348 & 1.294001 & -0.840829 \\
\hline 18 & 6 & 0 & -2.530397 & -0.731160 & 0.010468 \\
\hline 19 & 6 & 0 & -3.867743 & -0.783402 & 0.027668 \\
\hline 20 & 6 & 0 & -4.667012 & -2.049172 & 0.061559 \\
\hline 21 & 1 & 0 & -5.331168 & -2.116717 & -0.807495 \\
\hline 22 & 1 & 0 & -4.020354 & -2.931477 & 0.068373 \\
\hline 23 & 1 & 0 & -1.974411 & -1.668174 & 0.023590 \\
\hline 24 & 1 & 0 & -4.409334 & 0.161337 & 0.016814 \\
\hline 25 & 6 & 0 & -1.335649 & 2.641187 & -0.070546 \\
\hline 26 & 1 & 0 & -1.583990 & 3.695145 & -0.099472 \\
\hline 27 & 6 & 0 & -0.087719 & 2.085836 & -0.057100 \\
\hline 28 & 1 & 0 & 0.849709 & 2.619354 & -0.086762 \\
\hline 29 & 7 & 0 & -2.352406 & 1.698960 & -0.045650 \\
\hline 30 & 1 & 0 & -5.306348 & -2.084965 & 0.950856 \\
\hline
\end{tabular}

\section{Trienamine C15}

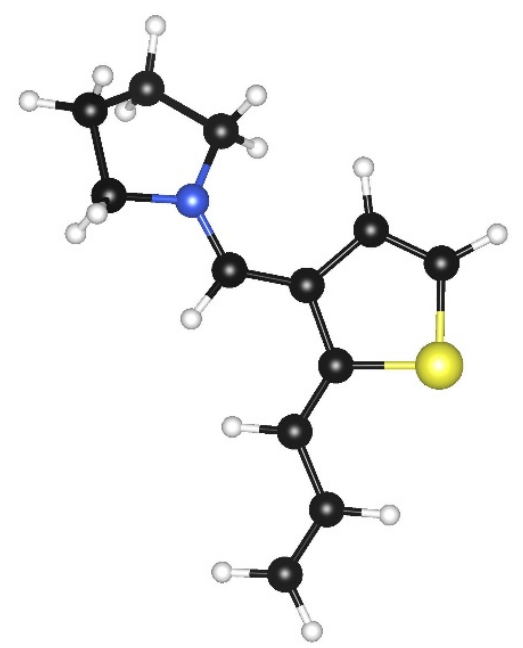

HF $(M 062 X / 6-31+G(d, p))=-918.9445892$ Hartrees

Imaginary Frequencies: none found 
Zero-point correction $=0.244232$ (Hartree/Particle)

Thermal correction $=0.200726$ Hartrees

Coordinates from last standard orientation:

\begin{tabular}{|c|c|c|c|c|c|}
\hline \multirow{2}{*}{$\begin{array}{l}\text { Center } \\
\text { Number }\end{array}$} & \multirow{2}{*}{$\begin{array}{l}\text { Atomic } \\
\text { Number }\end{array}$} & \multirow{2}{*}{$\begin{array}{c}\text { Atomic } \\
\text { Type }\end{array}$} & \multicolumn{3}{|c|}{ Coordinates (Angstroms) } \\
\hline & & & $\mathrm{x}$ & Y & z \\
\hline 1 & 6 & 0 & -0.740436 & 0.683344 & -0.037885 \\
\hline 2 & 6 & 0 & 0.547398 & 0.048767 & -0.048990 \\
\hline 3 & 6 & 0 & 1.692357 & 0.788243 & -0.058939 \\
\hline 4 & 1 & 0 & 1.623839 & 1.875642 & -0.059017 \\
\hline 5 & 7 & 0 & 2.957888 & 0.308802 & -0.062584 \\
\hline 6 & 6 & 0 & 4.150857 & 1.148229 & -0.050330 \\
\hline 7 & 1 & 0 & 4.018509 & 1.995909 & 0.629642 \\
\hline 8 & 1 & 0 & 4.368689 & 1.541392 & -1.054648 \\
\hline 9 & 6 & 0 & 5.241505 & 0.178446 & 0.407392 \\
\hline 10 & 1 & 0 & 5.234725 & 0.105091 & 1.500115 \\
\hline 11 & 1 & 0 & 6.239378 & 0.486356 & 0.088029 \\
\hline 12 & 6 & 0 & 4.790368 & -1.150354 & -0.212412 \\
\hline 13 & 1 & 0 & 5.237992 & -2.023656 & 0.266212 \\
\hline 14 & 1 & 0 & 5.053420 & -1.172964 & -1.275271 \\
\hline 15 & 6 & 0 & 3.265590 & -1.113281 & -0.056648 \\
\hline 16 & 1 & 0 & 2.744835 & -1.625276 & -0.875539 \\
\hline 17 & 1 & 0 & 2.933128 & -1.567989 & 0.888939 \\
\hline 18 & 1 & 0 & 0.606224 & -1.035439 & -0.045350 \\
\hline 19 & 1 & 0 & -0.729488 & 1.773411 & -0.045245 \\
\hline 20 & 6 & 0 & -1.952550 & 0.067424 & -0.017329 \\
\hline 21 & 6 & 0 & -3.242343 & 0.799549 & -0.008205 \\
\hline 22 & 6 & 0 & -2.181262 & -1.373105 & 0.008261 \\
\hline 23 & 6 & 0 & -3.476337 & -1.725308 & 0.047238 \\
\hline 24 & 1 & 0 & -1.377290 & -2.099335 & 0.000643 \\
\hline 25 & 1 & 0 & -3.876069 & -2.730608 & 0.074935 \\
\hline 26 & 6 & 0 & -3.462669 & 2.122614 & -0.043831 \\
\hline 27 & 1 & 0 & -4.466077 & 2.531933 & -0.030974 \\
\hline 28 & 1 & 0 & -2.638204 & 2.825801 & -0.088129 \\
\hline 29 & 16 & 0 & -4.592105 & -0.368080 & 0.055863 \\
\hline
\end{tabular}

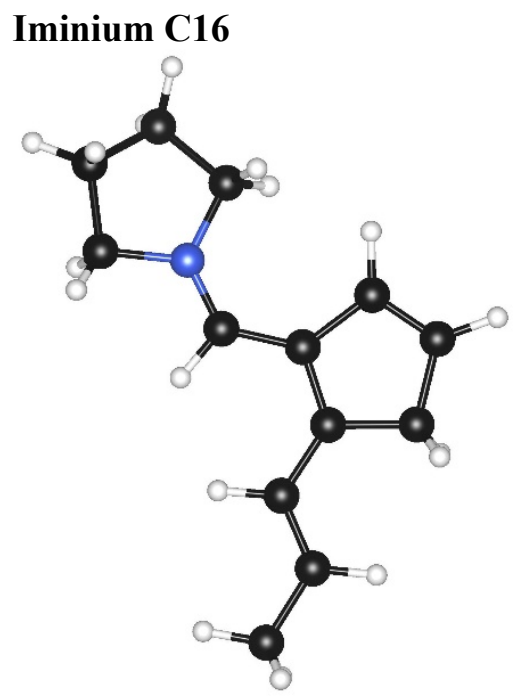


HF $(M 062 X / 6-31+G(d, p))=-560.4866441$ Hartrees

Imaginary Frequencies: none found

Zero-point correction $=0.284901$ (Hartree/Particle)

Thermal correction $=0.243456$ Hartrees

Coordinates from last standard orientation:

\begin{tabular}{|c|c|c|c|c|c|}
\hline \multirow{2}{*}{$\begin{array}{l}\text { Center } \\
\text { Number }\end{array}$} & \multirow{2}{*}{$\begin{array}{l}\text { Atomic } \\
\text { Number }\end{array}$} & \multirow{2}{*}{$\begin{array}{c}\text { Atomic } \\
\text { Type }\end{array}$} & \multicolumn{3}{|c|}{ Coordinates (Angstroms) } \\
\hline & & & $x$ & Y & Z \\
\hline 1 & 6 & 0 & -1.028839 & -0.593724 & 0.029855 \\
\hline 2 & 6 & 0 & 0.194275 & 0.021660 & 0.040183 \\
\hline 3 & 6 & 0 & 1.366295 & -0.773820 & 0.064398 \\
\hline 4 & 1 & 0 & 1.262495 & -1.858939 & 0.071190 \\
\hline 5 & 7 & 0 & 2.587056 & -0.322542 & 0.074237 \\
\hline 6 & 6 & 0 & 3.791755 & -1.186755 & 0.074070 \\
\hline 7 & 1 & 0 & 3.621171 & -2.056177 & -0.563209 \\
\hline 8 & 1 & 0 & 3.980284 & -1.518985 & 1.100891 \\
\hline 9 & 6 & 0 & 4.886649 & -0.242786 & -0.414686 \\
\hline 10 & 1 & 0 & 4.869153 & -0.179658 & -1.507234 \\
\hline 11 & 1 & 0 & 5.878268 & -0.574330 & -0.104655 \\
\hline 12 & 6 & 0 & 4.477620 & 1.096044 & 0.210229 \\
\hline 13 & 1 & 0 & 4.942651 & 1.955517 & -0.273784 \\
\hline 14 & 1 & 0 & 4.744994 & 1.116615 & 1.270988 \\
\hline 15 & 6 & 0 & 2.955231 & 1.112281 & 0.053190 \\
\hline 16 & 1 & 0 & 2.435646 & 1.637717 & 0.857971 \\
\hline 17 & 1 & 0 & 2.642880 & 1.531673 & -0.909654 \\
\hline 18 & 1 & 0 & 0.275447 & 1.103047 & 0.024627 \\
\hline 19 & 1 & 0 & -1.047607 & -1.683068 & 0.046560 \\
\hline 20 & 6 & 0 & -2.290377 & 0.072602 & -0.000969 \\
\hline 21 & 6 & 0 & -3.508027 & -0.565760 & -0.006145 \\
\hline 22 & 6 & 0 & -2.512724 & 1.532171 & -0.033646 \\
\hline 23 & 6 & 0 & -3.831108 & 1.776366 & -0.058116 \\
\hline 24 & 1 & 0 & -1.728677 & 2.279622 & -0.038183 \\
\hline 25 & 1 & 0 & -4.306914 & 2.748222 & -0.086281 \\
\hline 26 & 6 & 0 & -3.820659 & -2.020982 & 0.020108 \\
\hline 27 & 1 & 0 & -4.419549 & -2.292877 & -0.855662 \\
\hline 28 & 1 & 0 & -2.932981 & -2.654448 & 0.035185 \\
\hline 29 & 6 & 0 & -4.584474 & 0.477522 & -0.043134 \\
\hline 30 & 1 & 0 & -5.251094 & 0.385197 & 0.825951 \\
\hline 31 & 1 & 0 & -5.223914 & 0.349565 & -0.928072 \\
\hline 32 & 1 & 0 & -4.425354 & -2.259822 & 0.901683 \\
\hline
\end{tabular}




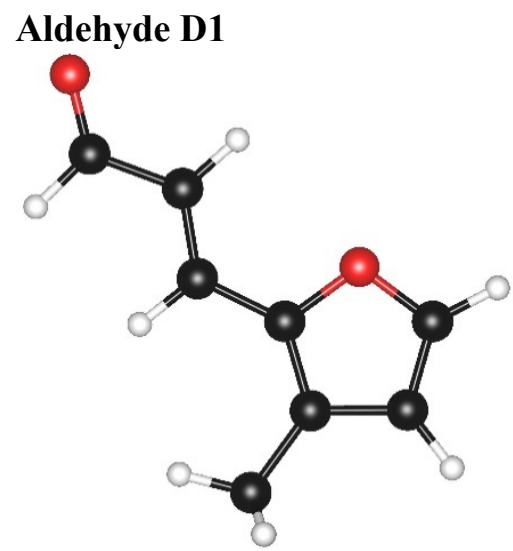

HF $(M 062 X / 6-31+G(d, p))=-459.8979071$ Hartrees

Imaginary Frequencies: none found

Zero-point correction $=0.142232$ (Hartree/Particle $)$

Thermal correction $=0.107220$ Hartrees

Coordinates from last standard orientation:

\begin{tabular}{|c|c|c|c|c|c|}
\hline \multirow{2}{*}{$\begin{array}{l}\text { Center } \\
\text { Number }\end{array}$} & \multirow{2}{*}{$\begin{array}{l}\text { Atomic } \\
\text { Number }\end{array}$} & \multirow{2}{*}{$\begin{array}{c}\text { Atomic } \\
\text { Type }\end{array}$} & \multicolumn{3}{|c|}{ Coordinates (Angstroms) } \\
\hline & & & $\mathrm{x}$ & Y & Z \\
\hline 1 & 6 & 0 & -0.102481 & 0.233702 & 0.000662 \\
\hline 2 & 6 & 0 & 1.220485 & -0.152536 & -0.000293 \\
\hline 3 & 6 & 0 & 1.732705 & -1.521036 & -0.000535 \\
\hline 4 & 1 & 0 & 0.973475 & -2.327786 & -0.001345 \\
\hline 5 & 6 & 0 & -1.344428 & -0.515975 & 0.001103 \\
\hline 6 & 6 & 0 & -2.559712 & 0.047555 & -0.000951 \\
\hline 7 & 6 & 0 & -3.843060 & -0.720904 & -0.000307 \\
\hline 8 & 1 & 0 & -4.445198 & -0.469280 & -0.879852 \\
\hline 9 & 1 & 0 & -3.664479 & -1.798944 & 0.000203 \\
\hline 10 & 1 & 0 & -1.244975 & -1.598792 & 0.003158 \\
\hline 11 & 1 & 0 & -2.629402 & 1.134185 & -0.003093 \\
\hline 12 & 6 & 0 & 1.104095 & 2.068347 & 0.000042 \\
\hline 13 & 1 & 0 & 1.192255 & 3.143044 & 0.000020 \\
\hline 14 & 6 & 0 & 1.999456 & 1.054762 & -0.000831 \\
\hline 15 & 1 & 0 & 3.076803 & 1.120251 & -0.001701 \\
\hline 16 & 1 & 0 & -4.444698 & -0.468471 & 0.879380 \\
\hline 17 & 8 & 0 & -0.172167 & 1.582452 & 0.000942 \\
\hline 18 & 8 & 0 & 2.915149 & -1.794664 & 0.000293 \\
\hline
\end{tabular}




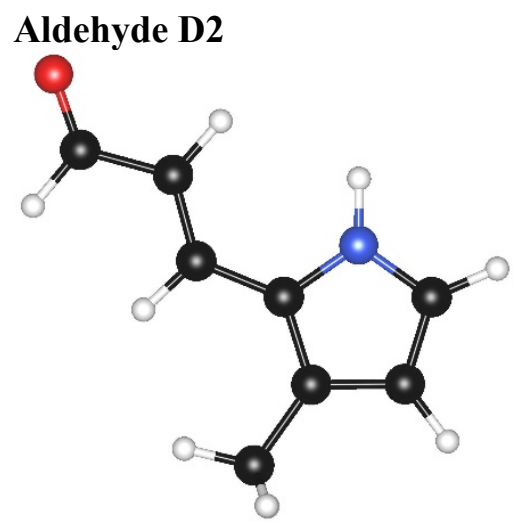

HF $(M 062 X / 6-31+G(d, p))=-440.0507199$ Hartrees

Imaginary Frequencies: none found

Zero-point correction $=0.154902$ (Hartree/Particle)

Thermal correction $=0.118945$ Hartrees

Coordinates from last standard orientation:

\begin{tabular}{|c|c|c|c|c|c|}
\hline \multirow{2}{*}{$\begin{array}{l}\text { Center } \\
\text { Number }\end{array}$} & \multirow{2}{*}{$\begin{array}{l}\text { Atomic } \\
\text { Number }\end{array}$} & \multirow{2}{*}{$\begin{array}{c}\text { Atomic } \\
\text { Type }\end{array}$} & \multicolumn{3}{|c|}{ Coordinates (Angstroms) } \\
\hline & & & $\mathrm{x}$ & Y & Z \\
\hline 1 & 6 & 0 & -0.105054 & 0.294259 & 0.006244 \\
\hline 2 & 6 & 0 & 1.210306 & -0.174614 & 0.001134 \\
\hline 3 & 6 & 0 & 1.629481 & -1.571959 & -0.000469 \\
\hline 4 & 1 & 0 & 0.818978 & -2.328335 & 0.002384 \\
\hline 5 & 6 & 0 & -1.360766 & -0.443665 & 0.011096 \\
\hline 6 & 6 & 0 & -2.590663 & 0.086088 & -0.010487 \\
\hline 7 & 6 & 0 & -3.854805 & -0.717756 & -0.002341 \\
\hline 8 & 1 & 0 & -4.460024 & -0.505008 & -0.890045 \\
\hline 9 & 1 & 0 & -3.639599 & -1.788958 & 0.018323 \\
\hline 10 & 1 & 0 & -1.262397 & -1.526477 & 0.033873 \\
\hline 11 & 1 & 0 & -2.717368 & 1.169269 & -0.036964 \\
\hline 12 & 6 & 0 & 1.284370 & 2.070152 & -0.000962 \\
\hline 13 & 1 & 0 & 1.532334 & 3.120735 & -0.001999 \\
\hline 14 & 6 & 0 & 2.079378 & 0.956498 & -0.003712 \\
\hline 15 & 1 & 0 & 3.158369 & 0.920876 & -0.008570 \\
\hline 16 & 1 & 0 & -4.469122 & -0.473160 & 0.870804 \\
\hline 17 & 8 & 0 & 2.790153 & -1.932376 & -0.004543 \\
\hline 18 & 7 & 0 & -0.030214 & 1.656994 & 0.004617 \\
\hline 19 & 1 & 0 & -0.824369 & 2.277093 & 0.013190 \\
\hline
\end{tabular}

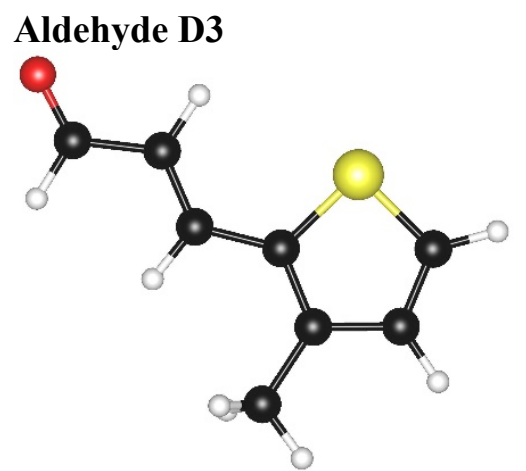


HF $(M 062 X / 6-31+G(d, p))=-782.8633054$ Hartrees

Imaginary Frequencies: none found

Zero-point correction $=0.138643$ (Hartree/Particle)

Thermal correction $=0.102203$ Hartrees

Coordinates from last standard orientation:

\begin{tabular}{|c|c|c|c|c|c|}
\hline \multirow{2}{*}{$\begin{array}{l}\text { Center } \\
\text { Number }\end{array}$} & \multirow{2}{*}{$\begin{array}{l}\text { Atomic } \\
\text { Number }\end{array}$} & \multirow{2}{*}{$\begin{array}{c}\text { Atomic } \\
\text { Type }\end{array}$} & \multicolumn{3}{|c|}{ Coordinates (Angstroms) } \\
\hline & & & $\mathrm{X}$ & Y & Z \\
\hline 1 & 6 & 0 & 0.125050 & -0.098312 & 0.005184 \\
\hline 2 & 6 & 0 & -1.157442 & 0.424254 & 0.001208 \\
\hline 3 & 6 & 0 & -1.481629 & 1.859008 & -0.000075 \\
\hline 4 & 1 & 0 & -0.630460 & 2.565087 & 0.002036 \\
\hline 5 & 6 & 0 & 1.392667 & 0.625986 & 0.010374 \\
\hline 6 & 6 & 0 & 2.609713 & 0.066859 & -0.010400 \\
\hline 7 & 6 & 0 & 3.891835 & 0.838414 & -0.002498 \\
\hline 8 & 1 & 0 & 4.491419 & 0.604729 & -0.888674 \\
\hline 9 & 1 & 0 & 3.707890 & 1.915461 & 0.016113 \\
\hline 10 & 1 & 0 & 1.321544 & 1.711153 & 0.033079 \\
\hline 11 & 1 & 0 & 2.697905 & -1.020084 & -0.034860 \\
\hline 12 & 6 & 0 & -1.662947 & -1.837838 & -0.003394 \\
\hline 13 & 1 & 0 & -2.192573 & -2.780246 & -0.006459 \\
\hline 14 & 6 & 0 & -2.177586 & -0.580968 & -0.003807 \\
\hline 15 & 1 & 0 & -3.233527 & -0.341085 & -0.007702 \\
\hline 16 & 1 & 0 & 4.497890 & 0.575842 & 0.871153 \\
\hline 17 & 8 & 0 & -2.619055 & 2.281731 & -0.003641 \\
\hline 18 & 16 & 0 & 0.065899 & -1.829320 & 0.004056 \\
\hline
\end{tabular}

\section{Enol D4}

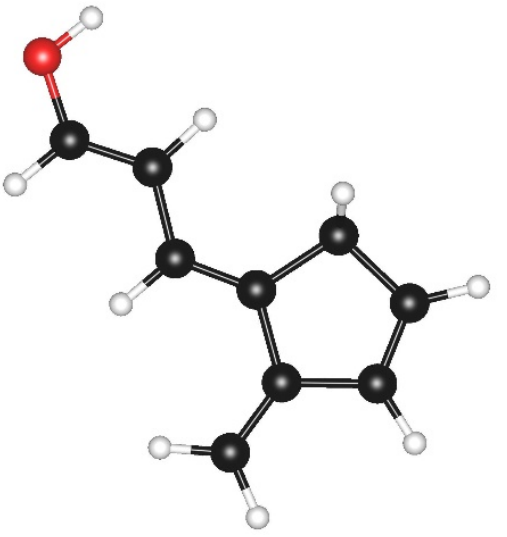

HF $(M 062 X / 6-31+G(d, p))=-423.9608766$ Hartrees

Imaginary Frequencies: none found

Zero-point correction $=0.165335$ (Hartree/Particle)

Thermal correction $=0.129713$ Hartrees

Coordinates from last standard orientation:

$\begin{array}{llll}\text { Center } & \text { Atomic } & \text { Atomic } & \text { Coordinates (Angstroms) } \\ \text { Number } & \text { Number } & \text { Type } & \text { Y } \\ \end{array}$




$\begin{array}{rrrrrr}1 & 6 & 0 & 0.307748 & 0.235456 & 0.000003 \\ 2 & 6 & 0 & -1.115973 & -0.173308 & 0.000008 \\ 3 & 6 & 0 & -1.581025 & -1.434547 & -0.000016 \\ 4 & 1 & 0 & -0.927563 & -2.299945 & -0.000044 \\ 5 & 6 & 0 & 1.388492 & -0.565664 & 0.000050 \\ 6 & 6 & 0 & 2.762105 & -0.085795 & 0.000024 \\ 7 & 6 & 0 & 3.835796 & -0.887779 & -0.000023 \\ 8 & 1 & 0 & 4.843590 & -0.487803 & -0.000031 \\ 9 & 1 & 0 & 3.729165 & -1.969592 & -0.000042 \\ 10 & 1 & 0 & 1.256974 & -1.647200 & 0.000096 \\ 11 & 1 & 0 & 2.910593 & 0.993208 & 0.000053 \\ 12 & 6 & 0 & -1.117312 & 2.142470 & 0.000008 \\ 13 & 1 & 0 & -1.458589 & 3.171293 & 0.000020 \\ 14 & 6 & 0 & -1.911786 & 1.063957 & 0.000031 \\ 15 & 1 & 0 & -2.996822 & 1.107156 & 0.000056 \\ 16 & 6 & 0 & 0.341184 & 1.758981 & -0.000048 \\ 17 & 1 & 0 & 0.862999 & 2.154564 & -0.881057 \\ 18 & 1 & 0 & 0.863107 & 2.154637 & 0.880862 \\ 19 & 8 & 0 & -2.885229 & -1.808447 & -0.000021 \\ 20 & 1 & 0 & -3.456991 & -1.031371 & 0.000030 \\ -------------------------------------------1\end{array}$

\section{Enol D5}

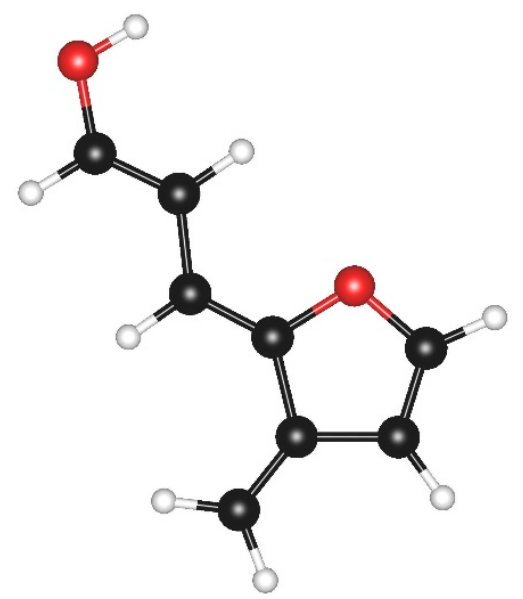

HF $(M 062 X / 6-31+G(d, p))=-459.860336$ Hartrees

Imaginary Frequencies: none found

Zero-point correction $=0.141606($ Hartree/Particle $)$

Thermal correction $=0.106401$ Hartrees

\section{Coordinates from last standard orientation:}

\begin{tabular}{cccccr} 
Center & Atomic & Atomic & \multicolumn{3}{c}{ Coordinates (Angstroms) } \\
Number & Number & Type & X & \multicolumn{2}{c}{ Y } \\
-1 & 6 & 0 & -0.290035 & 0.179621 & -0.000161 \\
2 & 6 & 0 & 1.148336 & -0.134971 & 0.000066 \\
3 & 6 & 0 & 1.691412 & -1.366053 & -0.000500 \\
4 & 1 & 0 & 1.092822 & -2.269758 & -0.001466 \\
5 & 6 & 0 & -1.385739 & -0.600844 & 0.000143 \\
6 & 6 & 0 & -2.743917 & -0.090076 & -0.000095 \\
7 & 6 & 0 & -3.835039 & -0.869159 & 0.000296
\end{tabular}




$\begin{array}{rrrrrr}8 & 1 & 0 & -4.832363 & -0.444134 & 0.000123 \\ 9 & 1 & 0 & -3.755690 & -1.953254 & 0.000823 \\ 10 & 1 & 0 & -1.241109 & -1.677662 & 0.000632 \\ 11 & 1 & 0 & -2.855529 & 0.991872 & -0.000602 \\ 12 & 6 & 0 & 0.845586 & 2.094389 & 0.000045 \\ 13 & 1 & 0 & 0.884677 & 3.173895 & -0.000063 \\ 14 & 6 & 0 & 1.815253 & 1.170643 & 0.000474 \\ 15 & 1 & 0 & 2.875187 & 1.380781 & 0.000739 \\ 16 & 8 & 0 & -0.412649 & 1.561089 & -0.000437 \\ 17 & 8 & 0 & 3.015761 & -1.645232 & -0.000046 \\ 18 & 1 & 0 & 3.531967 & -0.829893 & 0.002076 \\ --------------1\end{array}$

\section{Enol D6}

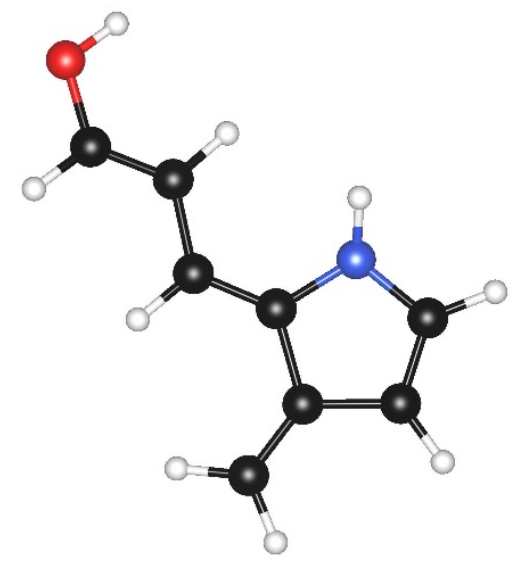

HF $(M 062 X / 6-31+G(d, p))=-440.0055622$ Hartrees

Imaginary Frequencies: none found

Zero-point correction $=0.153962$ (Hartree/Particle)

Thermal correction $=0.118574$ Hartrees

\section{Coordinates from last standard orientation:}

\begin{tabular}{|c|c|c|c|c|c|}
\hline \multirow{2}{*}{$\begin{array}{l}\text { Center } \\
\text { Number }\end{array}$} & \multirow{2}{*}{$\begin{array}{l}\text { Atomic } \\
\text { Number }\end{array}$} & \multirow{2}{*}{$\begin{array}{c}\text { Atomic } \\
\text { Type }\end{array}$} & \multicolumn{3}{|c|}{ Coordinates (Angstroms) } \\
\hline & & & $\mathrm{x}$ & $\mathrm{Y}$ & $\mathrm{Z}$ \\
\hline 1 & 6 & 0 & 2095607 & 0228053 & 1 \\
\hline$\perp$ & 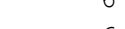 & 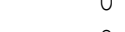 & -0.295001 & $0.2<0003$ & 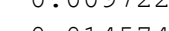 \\
\hline 2 & 6 & 0 & 1.137291 & -0.147054 & 0.014574 \\
\hline 3 & 6 & 0 & 1.612468 & -1.406036 & -0.037870 \\
\hline 4 & 1 & 0 & 0.969735 & -2.276401 & -0.105473 \\
\hline 5 & 6 & 0 & -1.395569 & -0.564194 & 0.027833 \\
\hline 6 & 6 & 0 & -2.759008 & -0.085899 & -0.044847 \\
\hline 7 & 6 & 0 & -3.851887 & -0.862197 & 0.039390 \\
\hline 8 & 1 & 0 & -4.849284 & -0.444479 & -0.034755 \\
\hline 9 & 1 & 0 & -3.768890 & -1.936384 & 0.181251 \\
\hline 10 & 1 & 0 & -1.247622 & -1.636430 & 0.117950 \\
\hline 11 & 1 & 0 & -2.910184 & 0.983763 & -0.194886 \\
\hline 12 & 6 & 0 & 0.997276 & 2.115899 & 0.019159 \\
\hline 13 & 1 & 0 & 1.169402 & 3.183394 & 0.024359 \\
\hline 14 & 6 & 0 & 1.893356 & 1.109258 & 0.058231 \\
\hline 15 & 1 & 0 & 2.966441 & 1.234070 & 0.084774 \\
\hline 16 & 8 & 0 & 2.920665 & -1.753140 & -0.019156 \\
\hline 17 & 1 & 0 & 3.472399 & -0.965558 & 0.062433 \\
\hline
\end{tabular}




\begin{tabular}{|c|c|c|c|c|c|}
\hline 18 & 7 & 0 & -0.297501 & 1.624841 & -0.070725 \\
\hline 19 & 1 & 0 & -1.114722 & 2.182290 & 0.112180 \\
\hline
\end{tabular}

\section{Enol D7}

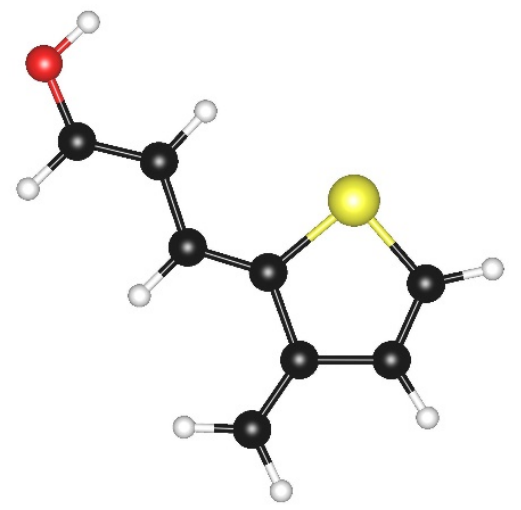

HF $(M 062 X / 6-31+G(d, p))=-440.0055622$ Hartrees

Imaginary Frequencies: none found

Zero-point correction $=0.153962$ (Hartree/Particle $)$

Thermal correction $=0.118574$ Hartrees

Coordinates from last standard orientation:

\begin{tabular}{|c|c|c|c|c|c|}
\hline \multirow{2}{*}{$\begin{array}{l}\text { Center } \\
\text { Number }\end{array}$} & \multirow{2}{*}{$\begin{array}{l}\text { Atomic } \\
\text { Number }\end{array}$} & \multirow{2}{*}{$\begin{array}{c}\text { Atomic } \\
\text { Type }\end{array}$} & \multicolumn{3}{|c|}{ Coordinates (Angstroms) } \\
\hline & & & $\mathrm{X}$ & Y & $\mathrm{z}$ \\
\hline 1 & 6 & 0 & -0.295607 & 0.228053 & -0.009722 \\
\hline 2 & 6 & 0 & 1.137291 & -0.147054 & 0.014574 \\
\hline 3 & 6 & 0 & 1.612468 & -1.406036 & -0.037870 \\
\hline 4 & 1 & 0 & 0.969735 & -2.276401 & -0.105473 \\
\hline 5 & 6 & 0 & -1.395569 & -0.564194 & 0.027833 \\
\hline 6 & 6 & 0 & -2.759008 & -0.085899 & -0.044847 \\
\hline 7 & 6 & 0 & -3.851887 & -0.862197 & 0.039390 \\
\hline 8 & 1 & 0 & -4.849284 & -0.444479 & -0.034755 \\
\hline 9 & 1 & 0 & -3.768890 & -1.936384 & 0.181251 \\
\hline 10 & 1 & 0 & -1.247622 & -1.636430 & 0.117950 \\
\hline 11 & 1 & 0 & -2.910184 & 0.983763 & -0.194886 \\
\hline 12 & 6 & 0 & 0.997276 & 2.115899 & 0.019159 \\
\hline 13 & 1 & 0 & 1.169402 & 3.183394 & 0.024359 \\
\hline 14 & 6 & 0 & 1.893356 & 1.109258 & 0.058231 \\
\hline 15 & 1 & 0 & 2.966441 & 1.234070 & 0.084774 \\
\hline 16 & 8 & 0 & 2.920665 & -1.753140 & -0.019156 \\
\hline 17 & 1 & 0 & 3.472399 & -0.965558 & 0.062433 \\
\hline 18 & 7 & 0 & -0.297501 & 1.624841 & -0.070725 \\
\hline 19 & 1 & 0 & -1.114722 & 2.182290 & 0.112180 \\
\hline
\end{tabular}




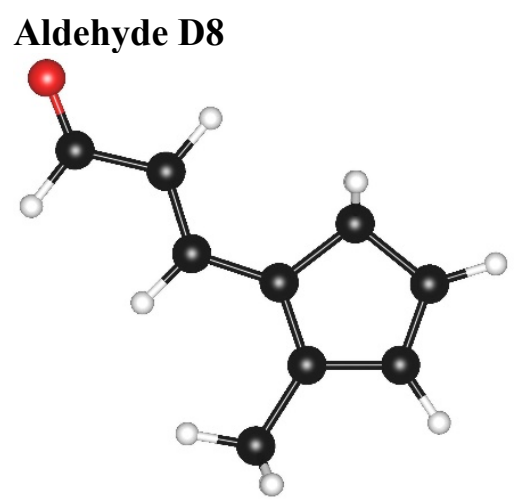

HF $(M 062 X / 6-31+G(d, p))=-423.9805038$ Hartrees

Imaginary Frequencies: none found

Zero-point correction $=0.164887$ (Hartree/Particle)

Thermal correction $=0.129622$ Hartrees

Coordinates from last standard orientation:

\begin{tabular}{|c|c|c|c|c|c|}
\hline \multirow{2}{*}{$\begin{array}{l}\text { Center } \\
\text { Number }\end{array}$} & \multirow{2}{*}{$\begin{array}{l}\text { Atomic } \\
\text { Number }\end{array}$} & \multirow{2}{*}{$\begin{array}{c}\text { Atomic } \\
\text { Type }\end{array}$} & \multicolumn{3}{|c|}{ Coordinates (Angstroms) } \\
\hline & & & $x$ & $Y$ & Z \\
\hline 1 & 6 & 0 & -0.113819 & 0.281663 & 0.000145 \\
\hline 2 & 6 & 0 & 1.162129 & -0.209571 & 0.000027 \\
\hline 3 & 6 & 0 & 1.559929 & -1.621378 & 0.000172 \\
\hline 4 & 1 & 0 & 0.744675 & -2.369977 & 0.000730 \\
\hline 5 & 6 & 0 & -1.360488 & -0.466393 & 0.000303 \\
\hline 6 & 6 & 0 & -2.579191 & 0.095678 & -0.000359 \\
\hline 7 & 6 & 0 & -3.865027 & -0.669881 & -0.000171 \\
\hline 8 & 1 & 0 & -4.467677 & -0.420157 & -0.880152 \\
\hline 9 & 1 & 0 & -3.685433 & -1.747835 & 0.000281 \\
\hline 10 & 1 & 0 & -1.292156 & -1.552852 & 0.001034 \\
\hline 11 & 1 & 0 & -2.660568 & 1.182951 & -0.001044 \\
\hline 12 & 6 & 0 & 1.448470 & 2.062390 & -0.000150 \\
\hline 13 & 1 & 0 & 1.872773 & 3.058718 & -0.000246 \\
\hline 14 & 6 & 0 & 2.122967 & 0.900346 & -0.000330 \\
\hline 15 & 1 & 0 & 3.196217 & 0.756685 & -0.000626 \\
\hline 16 & 6 & 0 & -0.030677 & 1.788382 & 0.000431 \\
\hline 17 & 1 & 0 & -0.527873 & 2.214458 & 0.881984 \\
\hline 18 & 1 & 0 & -0.528719 & 2.215069 & -0.880316 \\
\hline 19 & 1 & 0 & -4.467837 & -0.419462 & 0.879507 \\
\hline 20 & 8 & 0 & 2.718855 & -1.985626 & -0.000195 \\
\hline
\end{tabular}




\section{Iminium D9}

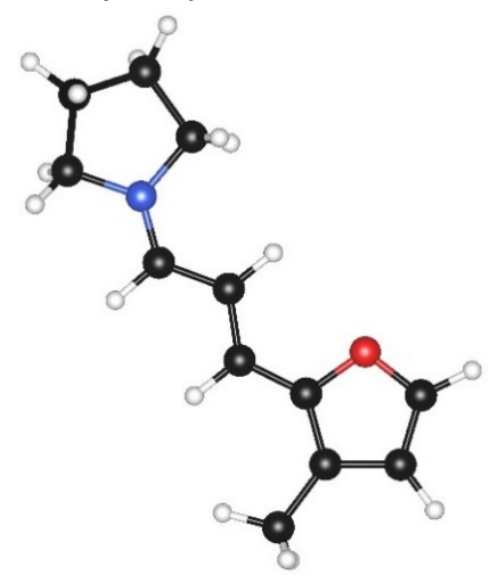

HF $(M 062 X / 6-31+G(d, p))=-596.3965876$ Hartrees

Imaginary Frequencies: none found

Zero-point correction $=0.262554$ (Hartree/Particle)

Thermal correction $=0.221869$ Hartrees

Coordinates from last standard orientation:

\begin{tabular}{|c|c|c|c|c|c|}
\hline \multirow{2}{*}{$\begin{array}{l}\text { Center } \\
\text { Number }\end{array}$} & \multirow{2}{*}{$\begin{array}{l}\text { Atomic } \\
\text { Number }\end{array}$} & \multirow{2}{*}{$\begin{array}{c}\text { Atomic } \\
\text { Type }\end{array}$} & \multicolumn{3}{|c|}{ Coordinates (Angstroms) } \\
\hline & & & $\mathrm{x}$ & Y & Z \\
\hline 1 & 6 & 0 & -1.691202 & 0.394021 & -0.012816 \\
\hline 2 & 6 & 0 & -0.303546 & 0.598035 & -0.018739 \\
\hline 3 & 6 & 0 & 0.596327 & -0.494073 & -0.007672 \\
\hline 4 & 1 & 0 & 0.167257 & -1.494035 & -0.045647 \\
\hline 5 & 7 & 0 & 1.895470 & -0.464442 & 0.037045 \\
\hline 6 & 6 & 0 & 2.734530 & -1.691582 & 0.011388 \\
\hline 7 & 1 & 0 & 2.310201 & -2.411734 & -0.689907 \\
\hline 8 & 1 & 0 & 2.744049 & -2.122550 & 1.018403 \\
\hline 9 & 6 & 0 & 4.109605 & -1.158707 & -0.378783 \\
\hline 10 & 1 & 0 & 4.173958 & -1.038696 & -1.464792 \\
\hline 11 & 1 & 0 & 4.909639 & -1.827159 & -0.058654 \\
\hline 12 & 6 & 0 & 4.149937 & 0.204240 & 0.320211 \\
\hline 13 & 1 & 0 & 4.905457 & 0.875793 & -0.088871 \\
\hline 14 & 1 & 0 & 4.349195 & 0.076851 & 1.388487 \\
\hline 15 & 6 & 0 & 2.736398 & 0.751832 & 0.113151 \\
\hline 16 & 1 & 0 & 2.387552 & 1.384245 & 0.932558 \\
\hline 17 & 1 & 0 & 2.641104 & 1.293913 & -0.834105 \\
\hline 18 & 6 & 0 & -2.504530 & -0.797624 & 0.028396 \\
\hline 19 & 6 & 0 & -3.848367 & -0.761915 & 0.013660 \\
\hline 20 & 6 & 0 & -4.721951 & -1.967653 & 0.057456 \\
\hline 21 & 1 & 0 & -5.372979 & -1.992958 & -0.822630 \\
\hline 22 & 1 & 0 & -4.143765 & -2.892735 & 0.096878 \\
\hline 23 & 1 & 0 & -1.988991 & -1.752404 & 0.077499 \\
\hline 24 & 1 & 0 & -4.342161 & 0.207859 & -0.032419 \\
\hline 25 & 6 & 0 & -1.354447 & 2.565597 & -0.078930 \\
\hline 26 & 1 & 0 & -1.731887 & 3.575213 & -0.114552 \\
\hline 27 & 6 & 0 & -0.116032 & 2.035754 & -0.062814 \\
\hline 28 & 1 & 0 & 0.800813 & 2.600663 & -0.093484 \\
\hline 29 & 1 & 0 & -5.380479 & -1.923668 & 0.931391 \\
\hline 30 & 8 & 0 & -2.302198 & 1.578118 & -0.045815 \\
\hline
\end{tabular}




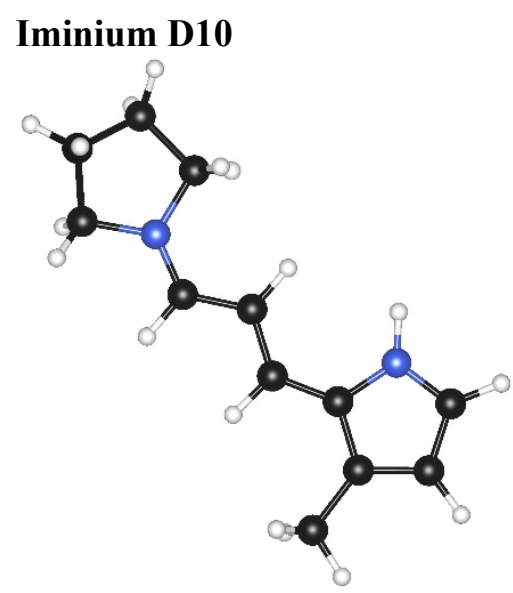

HF $(M 062 X / 6-31+G(d, p))=-576.5584271$ Hartrees

Imaginary Frequencies: none found

Zero-point correction $=0.275649$ (Hartree/Particle)

Thermal correction $=0.235029$ Hartrees

Coordinates from last standard orientation:

\begin{tabular}{|c|c|c|c|c|c|}
\hline \multirow{2}{*}{$\begin{array}{l}\text { Center } \\
\text { Number }\end{array}$} & \multirow{2}{*}{$\begin{array}{l}\text { Atomic } \\
\text { Number }\end{array}$} & \multirow{2}{*}{$\begin{array}{c}\text { Atomic } \\
\text { Type }\end{array}$} & \multicolumn{3}{|c|}{ Coordinates (Angstroms) } \\
\hline & & & $x$ & $\mathrm{Y}$ & Z \\
\hline & & & 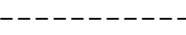 & -1 & ---------- \\
\hline 1 & 6 & 0 & -1.715324 & 0.467131 & 0.108580 \\
\hline 2 & 6 & 0 & -0.312458 & 0.656429 & 0.035958 \\
\hline 3 & 6 & 0 & 0.560096 & -0.454051 & 0.039368 \\
\hline 4 & 1 & 0 & 0.104197 & -1.443502 & 0.041747 \\
\hline 5 & 7 & 0 & 1.862572 & -0.460094 & 0.032756 \\
\hline 6 & 6 & 0 & 2.665810 & -1.709854 & 0.008394 \\
\hline 7 & 1 & 0 & 2.218387 & -2.421633 & -0.687271 \\
\hline 8 & 1 & 0 & 2.670585 & -2.138165 & 1.016632 \\
\hline 9 & 6 & 0 & 4.054053 & -1.220157 & -0.394833 \\
\hline 10 & 1 & 0 & 4.118140 & -1.121460 & -1.483010 \\
\hline 11 & 1 & 0 & 4.837344 & -1.903678 & -0.065053 \\
\hline 12 & 6 & 0 & 4.133108 & 0.154659 & 0.277179 \\
\hline 13 & 1 & 0 & 4.905748 & 0.797151 & -0.146639 \\
\hline 14 & 1 & 0 & 4.331238 & 0.042922 & 1.347542 \\
\hline 15 & 6 & 0 & 2.733916 & 0.733427 & 0.062023 \\
\hline 16 & 1 & 0 & 2.406191 & 1.402124 & 0.861499 \\
\hline 17 & 1 & 0 & 2.646888 & 1.248840 & -0.901202 \\
\hline 18 & 6 & 0 & -2.500605 & -0.753312 & 0.250592 \\
\hline 19 & 6 & 0 & -3.759798 & -0.889143 & -0.190070 \\
\hline 20 & 6 & 0 & -4.588118 & -2.119556 & -0.018630 \\
\hline 21 & 1 & 0 & -4.913524 & -2.501169 & -0.991395 \\
\hline 22 & 1 & 0 & -4.039424 & -2.905673 & 0.503848 \\
\hline 23 & 1 & 0 & -2.022684 & -1.590694 & 0.753210 \\
\hline 24 & 1 & 0 & -4.229628 & -0.063870 & -0.727491 \\
\hline 25 & 6 & 0 & -1.304906 & 2.673837 & -0.108544 \\
\hline 26 & 1 & 0 & -1.579106 & 3.714586 & -0.187584 \\
\hline 27 & 6 & 0 & -0.083146 & 2.074954 & -0.104125 \\
\hline 28 & 1 & 0 & 0.855603 & 2.593148 & -0.209052 \\
\hline 29 & 1 & 0 & -5.494926 & -1.890597 & 0.550732 \\
\hline 30 & 7 & 0 & -2.269776 & 1.692125 & 0.025655 \\
\hline 31 & 1 & 0 & -3.260362 & 1.871282 & 0.119262 \\
\hline
\end{tabular}




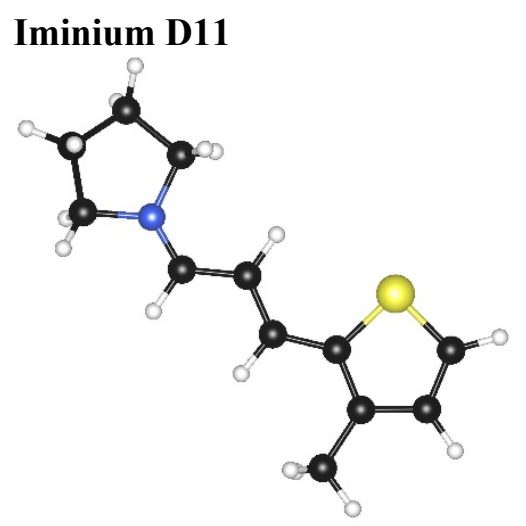

HF $(M 062 X / 6-31+G(d, p))=-919.3596831$ Hartrees Imaginary Frequencies: none found Zero-point correction $=0.258779$ (Hartree/Particle)

Thermal correction $=0.216453$ Hartrees

Coordinates from last standard orientation:

\begin{tabular}{|c|c|c|c|c|c|}
\hline \multirow{2}{*}{$\begin{array}{l}\text { Center } \\
\text { Number }\end{array}$} & \multirow{2}{*}{$\begin{array}{l}\text { Atomic } \\
\text { Number }\end{array}$} & \multirow{2}{*}{$\begin{array}{c}\text { Atomic } \\
\text { Type }\end{array}$} & \multicolumn{3}{|c|}{ Coordinates (Angstroms) } \\
\hline & & & $\mathrm{x}$ & $\mathrm{Y}$ & Z \\
\hline 1 & 6 & 0 & -1.570318 & 0.231180 & 0.100240 \\
\hline 2 & 6 & 0 & -0.202107 & 0.550798 & 0.016608 \\
\hline 3 & 6 & 0 & 0.744852 & -0.512991 & -0.002921 \\
\hline 4 & 1 & 0 & 0.342243 & -1.523576 & -0.046115 \\
\hline 5 & 7 & 0 & 2.043341 & -0.458791 & 0.010814 \\
\hline 6 & 6 & 0 & 2.891327 & -1.682015 & -0.046452 \\
\hline 7 & 1 & 0 & 2.482309 & -2.376714 & -0.781534 \\
\hline 8 & 1 & 0 & 2.881658 & -2.149353 & 0.944114 \\
\hline 9 & 6 & 0 & 4.272200 & -1.134237 & -0.389693 \\
\hline 10 & 1 & 0 & 4.363483 & -0.986638 & -1.470345 \\
\hline 11 & 1 & 0 & 5.066727 & -1.807939 & -0.066854 \\
\hline 12 & 6 & 0 & 4.289813 & 0.211075 & 0.343229 \\
\hline 13 & 1 & 0 & 5.054136 & 0.894363 & -0.028494 \\
\hline 14 & 1 & 0 & 4.458034 & 0.059879 & 1.413841 \\
\hline 15 & 6 & 0 & 2.882025 & 0.758086 & 0.105113 \\
\hline 16 & 1 & 0 & 2.511155 & 1.393444 & 0.912280 \\
\hline 17 & 1 & 0 & 2.814315 & 1.296144 & -0.846673 \\
\hline 18 & 6 & 0 & -2.191145 & -1.079997 & 0.254689 \\
\hline 19 & 6 & 0 & -3.427289 & -1.378756 & -0.176790 \\
\hline 20 & 6 & 0 & -4.087777 & -2.703986 & 0.003423 \\
\hline 21 & 1 & 0 & -4.362173 & -3.129434 & -0.967207 \\
\hline 22 & 1 & 0 & -3.444743 & -3.411183 & 0.531381 \\
\hline 23 & 1 & 0 & -1.616459 & -1.848198 & 0.767670 \\
\hline 24 & 1 & 0 & -4.001400 & -0.616280 & -0.704811 \\
\hline 25 & 6 & 0 & -1.142936 & 2.670485 & -0.146636 \\
\hline 26 & 1 & 0 & -1.272210 & 3.738779 & -0.252908 \\
\hline 27 & 6 & 0 & 0.017578 & 1.970910 & -0.128059 \\
\hline 28 & 1 & 0 & 0.978034 & 2.451041 & -0.241286 \\
\hline 29 & 1 & 0 & -5.017971 & -2.587092 & 0.569381 \\
\hline 30 & 16 & 0 & -2.524866 & 1.650686 & 0.037209 \\
\hline
\end{tabular}




\section{Trienamine D12}

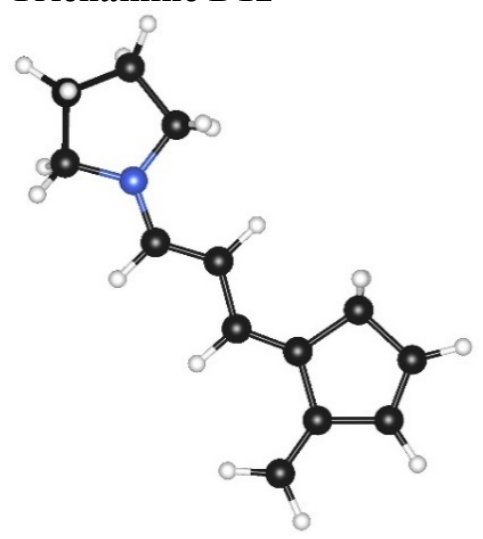

HF $(M 062 X / 6-31+G(d, p))=-560.073306$ Hartrees

Imaginary Frequencies: none found

Zero-point correction $=0.271512$ (Hartree/Particle)

Thermal correction $=0.232085$ Hartrees

Coordinates from last standard orientation:

\begin{tabular}{|c|c|c|c|c|c|}
\hline \multirow{2}{*}{$\begin{array}{l}\text { Center } \\
\text { Number }\end{array}$} & \multirow{2}{*}{$\begin{array}{l}\text { Atomic } \\
\text { Number }\end{array}$} & \multirow{2}{*}{$\begin{array}{c}\text { Atomic } \\
\text { Type }\end{array}$} & \multicolumn{3}{|c|}{ Coordinates (Angstroms) } \\
\hline & & & $\mathrm{x}$ & $\mathrm{Y}$ & Z \\
\hline & & & (5) & 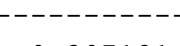 & ---------- \\
\hline 1 & 6 & 0 & -1.827852 & 0.307121 & -0.044236 \\
\hline 2 & 6 & 0 & -0.369781 & 0.541339 & -0.108042 \\
\hline 3 & 6 & 0 & 0.548475 & -0.454960 & -0.230683 \\
\hline 4 & 1 & 0 & 0.179870 & -1.471602 & -0.363203 \\
\hline 5 & 7 & 0 & 1.909509 & -0.376128 & -0.225989 \\
\hline 6 & 6 & 0 & 2.729982 & -1.574981 & -0.389007 \\
\hline 7 & 1 & 0 & 2.531914 & -2.054097 & -1.353162 \\
\hline 8 & 1 & 0 & 2.518349 & -2.304876 & 0.408446 \\
\hline 9 & 6 & 0 & 4.157111 & -1.040336 & -0.275414 \\
\hline 10 & 1 & 0 & 4.486988 & -0.649786 & -1.244033 \\
\hline 11 & 1 & 0 & 4.866153 & -1.806099 & 0.046405 \\
\hline 12 & 6 & 0 & 4.003289 & 0.106225 & 0.730367 \\
\hline 13 & 1 & 0 & 4.830875 & 0.818540 & 0.705944 \\
\hline 14 & 1 & 0 & 3.929227 & -0.298308 & 1.745606 \\
\hline 15 & 6 & 0 & 2.668397 & 0.740677 & 0.327816 \\
\hline 16 & 1 & 0 & 2.129762 & 1.175441 & 1.177995 \\
\hline 17 & 1 & 0 & 2.815806 & 1.529372 & -0.423448 \\
\hline 18 & 6 & 0 & -2.471286 & -0.871883 & 0.084874 \\
\hline 19 & 6 & 0 & -3.916767 & -1.014796 & 0.118382 \\
\hline 20 & 6 & 0 & -4.560359 & -2.185751 & 0.240247 \\
\hline 21 & 1 & 0 & -5.643159 & -2.239386 & 0.260034 \\
\hline 22 & 1 & 0 & -4.014014 & -3.122157 & 0.321912 \\
\hline 23 & 1 & 0 & -1.897521 & -1.793464 & 0.178189 \\
\hline 24 & 1 & 0 & -4.505457 & -0.101732 & 0.036190 \\
\hline 25 & 6 & 0 & -1.361238 & 2.638861 & -0.122039 \\
\hline 26 & 1 & 0 & -1.491799 & 3.714207 & -0.155218 \\
\hline 27 & 6 & 0 & -0.181787 & 1.999846 & -0.116530 \\
\hline 28 & 1 & 0 & 0.779814 & 2.496022 & -0.157334 \\
\hline 29 & 6 & 0 & -2.513519 & 1.666933 & -0.106781 \\
\hline 30 & 1 & 0 & -3.177486 & 1.825417 & 0.752131 \\
\hline
\end{tabular}




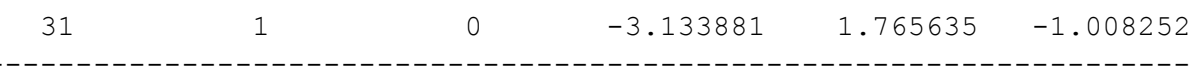

\section{Fulvene trienamine D12'}

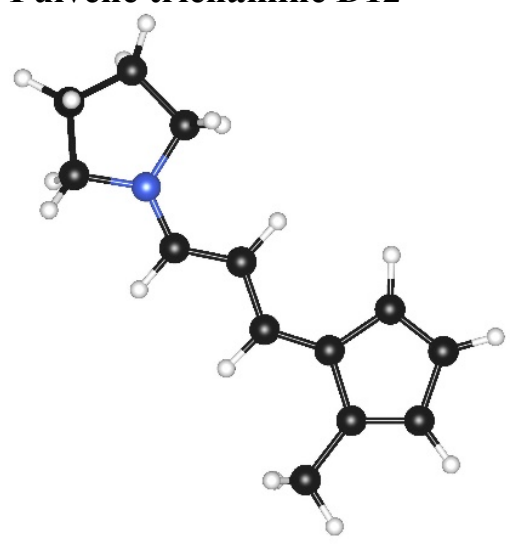

HF $(M 062 X / 6-31+G(d, p))=-560.0912156$ Hartrees

Imaginary Frequencies: none found

Zero-point correction $=0.271813$ (Hartree/Particle)

Thermal correction $=0.230195$ Hartrees

Coordinates from last standard orientation:

\begin{tabular}{|c|c|c|c|c|c|}
\hline \multirow{2}{*}{$\begin{array}{l}\text { Center } \\
\text { Number }\end{array}$} & \multirow{2}{*}{$\begin{array}{l}\text { Atomic } \\
\text { Number }\end{array}$} & \multirow{2}{*}{$\begin{array}{c}\text { Atomic } \\
\text { Type }\end{array}$} & \multicolumn{3}{|c|}{ Coordinates (Angstroms) } \\
\hline & & & $\mathrm{x}$ & Y & $\mathrm{z}$ \\
\hline & & & & & -------- \\
\hline 1 & 6 & 0 & -1.132369 & -0.547330 & 0.028544 \\
\hline 2 & 6 & 0 & 0.172321 & 0.040776 & 0.036827 \\
\hline 3 & 6 & 0 & 1.289492 & -0.742770 & 0.061365 \\
\hline 4 & 1 & 0 & 1.177782 & -1.826743 & 0.073689 \\
\hline 5 & 7 & 0 & 2.568300 & -0.313685 & 0.067162 \\
\hline 6 & 6 & 0 & 3.730101 & -1.198576 & 0.066680 \\
\hline 7 & 1 & 0 & 3.566911 & -2.046889 & -0.605195 \\
\hline 8 & 1 & 0 & 3.927305 & -1.588094 & 1.076092 \\
\hline 9 & 6 & 0 & 4.858394 & -0.275103 & -0.395063 \\
\hline 10 & 1 & 0 & 4.858583 & -0.210690 & -1.488324 \\
\hline 11 & 1 & 0 & 5.842190 & -0.618761 & -0.068909 \\
\hline 12 & 6 & 0 & 4.456515 & 1.075110 & 0.212129 \\
\hline 13 & 1 & 0 & 4.940178 & 1.926107 & -0.271335 \\
\hline 14 & 1 & 0 & 4.714836 & 1.096440 & 1.276125 \\
\hline 15 & 6 & 0 & 2.933025 & 1.097355 & 0.047304 \\
\hline 16 & 1 & 0 & 2.426237 & 1.637058 & 0.856143 \\
\hline 17 & 1 & 0 & 2.624809 & 1.551183 & -0.906094 \\
\hline 18 & 1 & 0 & 0.261487 & 1.122698 & 0.019997 \\
\hline 19 & 1 & 0 & -1.165068 & -1.639215 & 0.048955 \\
\hline 20 & 6 & 0 & -2.324065 & 0.111288 & -0.001303 \\
\hline 21 & 6 & 0 & -3.644430 & -0.533435 & -0.007354 \\
\hline 22 & 6 & 0 & -3.903273 & 1.753644 & -0.060399 \\
\hline 23 & 1 & 0 & -4.407674 & 2.712233 & -0.089997 \\
\hline 24 & 6 & 0 & -3.877234 & -2.012989 & 0.024040 \\
\hline 25 & 1 & 0 & -4.948165 & -2.230451 & 0.001535 \\
\hline 26 & 1 & 0 & -3.416804 & -2.514614 & -0.835080 \\
\hline 27 & 6 & 0 & -4.577746 & 0.455427 & -0.042327 \\
\hline 28 & 1 & 0 & -5.652407 & 0.311020 & -0.054757 \\
\hline 29 & 6 & 0 & -2.559581 & 1.554257 & -0.035735 \\
\hline
\end{tabular}




$\begin{array}{rrrrrr}30 & 1 & 0 & -1.791935 & 2.317623 & -0.042166 \\ 31 & 1 & 0 & -3.463250 & -2.469034 & 0.930932 \\ - & - & 0 & -\end{array}$

\section{Trienamine D13}

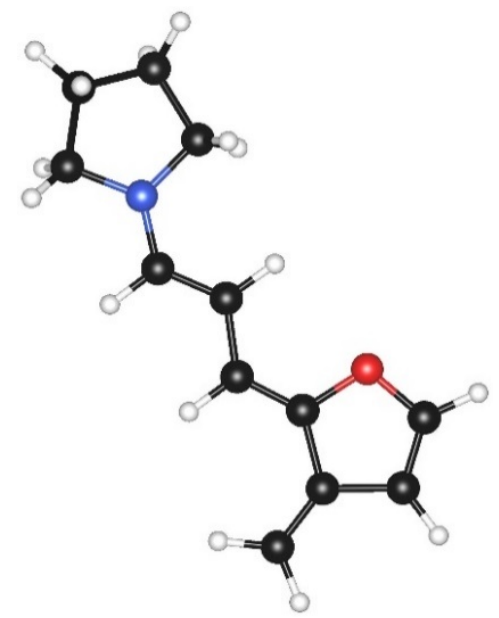

HF $(M 062 X / 6-31+G(d, p))=-595.9750923$ Hartrees

Imaginary Frequencies: none found

Zero-point correction $=0.248397$ (Hartree/Particle)

Thermal correction $=0.207832$ Hartrees

Coordinates from last standard orientation:

\begin{tabular}{|c|c|c|c|c|c|}
\hline \multirow{2}{*}{$\begin{array}{l}\text { Center } \\
\text { Number }\end{array}$} & \multirow{2}{*}{$\begin{array}{l}\text { Atomic } \\
\text { Number }\end{array}$} & \multirow{2}{*}{$\begin{array}{c}\text { Atomic } \\
\text { Type }\end{array}$} & \multicolumn{3}{|c|}{ Coordinates (Angstroms) } \\
\hline & & & $\mathrm{X}$ & Y & z \\
\hline & & & & & 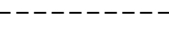 \\
\hline 1 & 6 & 0 & 1.797682 & 0.277345 & 0.029812 \\
\hline 2 & 6 & 0 & 0.348016 & 0.508579 & 0.077283 \\
\hline 3 & 6 & 0 & -0.566238 & -0.498594 & 0.155267 \\
\hline 4 & 1 & 0 & -0.191104 & -1.516858 & 0.243521 \\
\hline 5 & 7 & 0 & -1.920853 & -0.416906 & 0.148842 \\
\hline 6 & 6 & 0 & -2.762880 & -1.607594 & 0.255292 \\
\hline 7 & 1 & 0 & -2.510516 & -2.181208 & 1.152461 \\
\hline 8 & 1 & 0 & -2.629705 & -2.259802 & -0.621717 \\
\hline 9 & 6 & 0 & -4.176118 & -1.027483 & 0.296687 \\
\hline 10 & 1 & 0 & -4.423209 & -0.723444 & 1.319425 \\
\hline 11 & 1 & 0 & -4.930561 & -1.740239 & -0.042727 \\
\hline 12 & 6 & 0 & -4.056776 & 0.204845 & -0.607008 \\
\hline 13 & 1 & 0 & -4.855934 & 0.933379 & -0.455248 \\
\hline 14 & 1 & 0 & -4.069347 & -0.102875 & -1.658021 \\
\hline 15 & 6 & 0 & -2.676749 & 0.765811 & -0.249525 \\
\hline 16 & 1 & 0 & -2.186144 & 1.258137 & -1.096906 \\
\hline 17 & 1 & 0 & -2.739684 & 1.486364 & 0.578314 \\
\hline 18 & 6 & 0 & 2.536750 & -0.849373 & -0.074597 \\
\hline 19 & 6 & 0 & 3.984544 & -0.866065 & -0.081179 \\
\hline 20 & 6 & 0 & 4.732993 & -1.975679 & -0.191211 \\
\hline 21 & 1 & 0 & 5.816026 & -1.928216 & -0.190079 \\
\hline 22 & 1 & 0 & 4.277618 & -2.958337 & -0.285765 \\
\hline 23 & 1 & 0 & 2.012351 & -1.796474 & -0.165483 \\
\hline 24 & 1 & 0 & 4.476688 & 0.100107 & 0.011485 \\
\hline 25 & 6 & 0 & 1.448436 & 2.473715 & 0.129376 \\
\hline 26 & 1 & 0 & 1.810686 & 3.489859 & 0.175958 \\
\hline
\end{tabular}




\begin{tabular}{|c|c|c|c|c|c|}
\hline 27 & 6 & 0 & 0.208780 & 1.966524 & 0.112985 \\
\hline 28 & 1 & 0 & -0.699600 & 2.545863 & 0.159158 \\
\hline 29 & 8 & 0 & 2.422219 & 1.509987 & 0.089329 \\
\hline
\end{tabular}

\section{Trienamine D14}

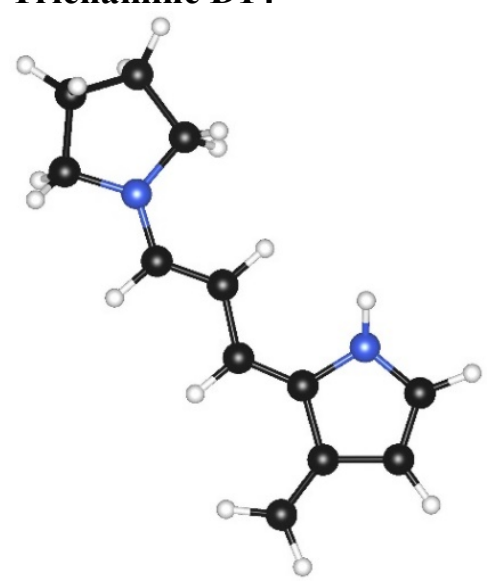

HF $(M 062 X / 6-31+G(d, p))=-576.1195168$ Hartrees

Imaginary Frequencies: none found

Zero-point correction $=0.260292$ (Hartree/Particle)

Thermal correction $=0.218647$ Hartrees

\section{Coordinates from last standard orientation:}

\begin{tabular}{|c|c|c|c|c|c|}
\hline \multirow{2}{*}{$\begin{array}{l}\text { Center } \\
\text { Number }\end{array}$} & \multirow{2}{*}{$\begin{array}{l}\text { Atomic } \\
\text { Number }\end{array}$} & \multirow{2}{*}{$\begin{array}{c}\text { Atomic } \\
\text { Type }\end{array}$} & \multicolumn{3}{|c|}{ Coordinates (Angstroms) } \\
\hline & & & $\mathrm{X}$ & Y & $\mathrm{z}$ \\
\hline 1 & 6 & 0 & -1.810461 & 0.316915 & -0.034383 \\
\hline 2 & 6 & 0 & -0.354818 & 0.552447 & -0.068468 \\
\hline 3 & 6 & 0 & 0.545656 & -0.463681 & -0.202131 \\
\hline 4 & 1 & 0 & 0.157590 & -1.469790 & -0.355128 \\
\hline 5 & 7 & 0 & 1.900709 & -0.397330 & -0.182714 \\
\hline 6 & 6 & 0 & 2.729631 & -1.584096 & -0.388730 \\
\hline 7 & 1 & 0 & 2.508527 & -2.050918 & -1.353832 \\
\hline 8 & 1 & 0 & 2.547630 & -2.326488 & 0.403448 \\
\hline 9 & 6 & 0 & 4.153748 & -1.033379 & -0.309481 \\
\hline 10 & 1 & 0 & 4.455489 & -0.639631 & -1.285961 \\
\hline 11 & 1 & 0 & 4.878368 & -1.792021 & -0.006196 \\
\hline 12 & 6 & 0 & 4.015744 & 0.112785 & 0.699461 \\
\hline 13 & 1 & 0 & 4.835766 & 0.832526 & 0.651241 \\
\hline 14 & 1 & 0 & 3.971940 & -0.289704 & 1.717252 \\
\hline 15 & 6 & 0 & 2.666019 & 0.734211 & 0.329715 \\
\hline 16 & 1 & 0 & 2.147783 & 1.178683 & 1.186761 \\
\hline 17 & 1 & 0 & 2.784754 & 1.509948 & -0.439995 \\
\hline 18 & 6 & 0 & -2.499792 & -0.849051 & 0.122349 \\
\hline 19 & 6 & 0 & -3.935742 & -0.967312 & 0.036303 \\
\hline 20 & 6 & 0 & -4.643952 & -2.089881 & 0.258505 \\
\hline 21 & 1 & 0 & -5.722863 & -2.107526 & 0.156254 \\
\hline 22 & 1 & 0 & -4.153370 & -3.017354 & 0.542018 \\
\hline 23 & 1 & 0 & -1.934629 & -1.751935 & 0.333561 \\
\hline 24 & 1 & 0 & -4.492017 & -0.074859 & -0.255716 \\
\hline 25 & 6 & 0 & -1.404166 & 2.564316 & -0.132656 \\
\hline 26 & 1 & 0 & -1.681360 & 3.607893 & -0.185625 \\
\hline
\end{tabular}




\begin{tabular}{|c|c|c|c|c|c|}
\hline 27 & 6 & 0 & -0.177222 & 2.007402 & -0.079641 \\
\hline 28 & 1 & 0 & 0.752625 & 2.552505 & -0.117696 \\
\hline 29 & 7 & 0 & -2.389412 & 1.579512 & -0.170448 \\
\hline 30 & 1 & 0 & -3.343193 & 1.759356 & 0.096688 \\
\hline
\end{tabular}

\section{Fulvene trienamine D14,}

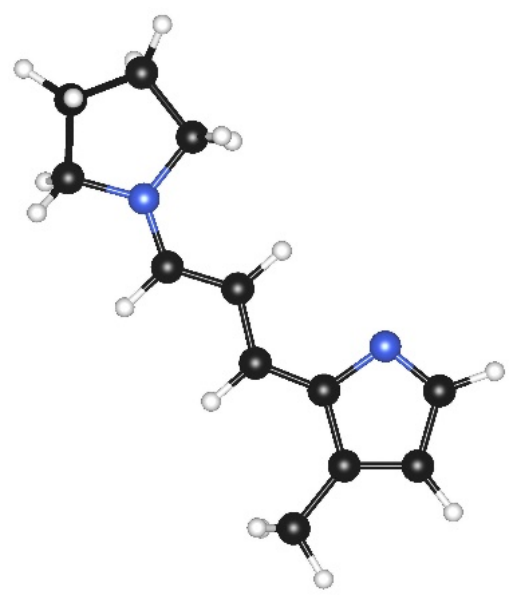

HF $(M 062 X / 6-31+G(d, p))=-576.1425105$ Hartrees

Imaginary Frequencies: none found

Zero-point correction $=0.260696$ (Hartree/Particle)

Thermal correction $=0.219094$ Hartrees

\section{Coordinates from last standard orientation:}

\begin{tabular}{|c|c|c|c|c|c|}
\hline \multirow{2}{*}{$\begin{array}{l}\text { Center } \\
\text { Number }\end{array}$} & \multirow{2}{*}{$\begin{array}{l}\text { Atomic } \\
\text { Number }\end{array}$} & \multirow{2}{*}{$\begin{array}{c}\text { Atomic } \\
\text { Type }\end{array}$} & \multicolumn{3}{|c|}{ Coordinates (Angstroms) } \\
\hline & & & $\mathrm{X}$ & Y & z \\
\hline & & & & & 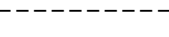 \\
\hline 1 & 6 & 0 & -1.141246 & -0.596119 & 0.031325 \\
\hline 2 & 6 & 0 & 0.149582 & 0.000656 & 0.039750 \\
\hline 3 & 6 & 0 & 1.274935 & -0.776097 & 0.066868 \\
\hline 4 & 1 & 0 & 1.176179 & -1.861574 & 0.081518 \\
\hline 5 & 7 & 0 & 2.544636 & -0.331557 & 0.072374 \\
\hline 6 & 6 & 0 & 3.719892 & -1.199588 & 0.072125 \\
\hline 7 & 1 & 0 & 3.565533 & -2.054286 & -0.593545 \\
\hline 8 & 1 & 0 & 3.925110 & -1.579071 & 1.083541 \\
\hline 9 & 6 & 0 & 4.833002 & -0.262274 & -0.398232 \\
\hline 10 & 1 & 0 & 4.827619 & -0.202290 & -1.491732 \\
\hline 11 & 1 & 0 & 5.822713 & -0.591388 & -0.075037 \\
\hline 12 & 6 & 0 & 4.414475 & 1.084367 & 0.205665 \\
\hline 13 & 1 & 0 & 4.885180 & 1.940083 & -0.282071 \\
\hline 14 & 1 & 0 & 4.675772 & 1.112860 & 1.268784 \\
\hline 15 & 6 & 0 & 2.890633 & 1.086302 & 0.044767 \\
\hline 16 & 1 & 0 & 2.376389 & 1.621970 & 0.850567 \\
\hline 17 & 1 & 0 & 2.572978 & 1.528400 & -0.910210 \\
\hline 18 & 1 & 0 & 0.201975 & 1.084358 & 0.020312 \\
\hline 19 & 1 & 0 & -1.193510 & -1.686367 & 0.051390 \\
\hline 20 & 6 & 0 & -2.322825 & 0.088962 & 0.000288 \\
\hline 21 & 6 & 0 & -3.664220 & -0.481480 & -0.010936 \\
\hline 22 & 6 & 0 & -3.659380 & 1.769595 & -0.057142 \\
\hline 23 & 1 & 0 & -4.005512 & 2.798977 & -0.084300 \\
\hline 24 & 6 & 0 & -4.001087 & -1.940113 & 0.015772 \\
\hline
\end{tabular}




$\begin{array}{rrrrrr}25 & 1 & 0 & -5.083429 & -2.085694 & -0.014318 \\ 26 & 1 & 0 & -3.566988 & -2.466631 & -0.841201 \\ 27 & 6 & 0 & -4.507846 & 0.589242 & -0.047762 \\ 28 & 1 & 0 & -5.590577 & 0.573713 & -0.065994 \\ 29 & 7 & 0 & -2.385025 & 1.489703 & -0.029087 \\ 30 & 1 & 0 & -3.622201 & -2.420807 & 0.924356 \\ -\end{array}$

\section{Trienamine D15}

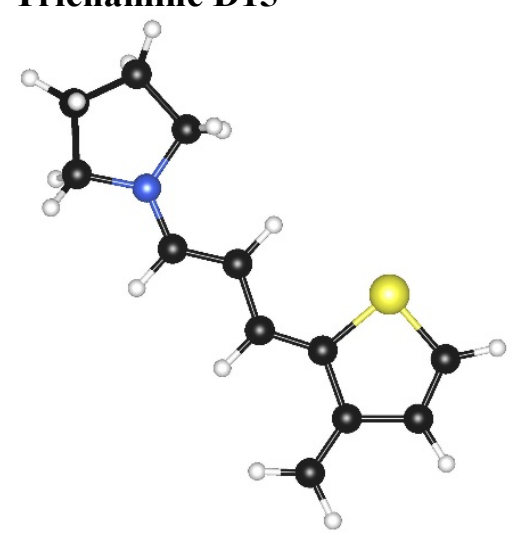

HF $(M 062 X / 6-31+G(d, p))=-918.9403592$ Hartrees

Imaginary Frequencies: none found

Zero-point correction $=0.244624$ (Hartree/Particle)

Thermal correction $=0.202883$ Hartrees

Coordinates from last standard orientation:

\begin{tabular}{|c|c|c|c|c|c|}
\hline \multirow{2}{*}{$\begin{array}{l}\text { Center } \\
\text { Number }\end{array}$} & \multirow{2}{*}{$\begin{array}{l}\text { Atomic } \\
\text { Number }\end{array}$} & \multirow{2}{*}{$\begin{array}{c}\text { Atomic } \\
\text { Type }\end{array}$} & \multicolumn{3}{|c|}{ Coordinates (Angstroms) } \\
\hline & & & $\mathrm{x}$ & Y & Z \\
\hline 1 & 6 & 0 & -1.640185 & 0.073354 & -0.027715 \\
\hline 2 & 6 & 0 & -0.215382 & 0.446174 & -0.092488 \\
\hline 3 & 6 & 0 & 0.753541 & -0.496227 & -0.296318 \\
\hline 4 & 1 & 0 & 0.435806 & -1.510709 & -0.534960 \\
\hline 5 & 7 & 0 & 2.101909 & -0.349431 & -0.254567 \\
\hline 6 & 6 & 0 & 2.987775 & -1.471173 & -0.575418 \\
\hline 7 & 1 & 0 & 2.852962 & -1.789304 & -1.613814 \\
\hline 8 & 1 & 0 & 2.770246 & -2.328589 & 0.079401 \\
\hline 9 & 6 & 0 & 4.383723 & -0.909852 & -0.304025 \\
\hline 10 & 1 & 0 & 4.747470 & -0.372512 & -1.186433 \\
\hline 11 & 1 & 0 & 5.105528 & -1.690992 & -0.056832 \\
\hline 12 & 6 & 0 & 4.133857 & 0.074114 & 0.843850 \\
\hline 13 & 1 & 0 & 4.934229 & 0.806313 & 0.969856 \\
\hline 14 & 1 & 0 & 4.015208 & -0.471934 & 1.785904 \\
\hline 15 & 6 & 0 & 2.803341 & 0.720849 & 0.451116 \\
\hline 16 & 1 & 0 & 2.209612 & 1.047790 & 1.311632 \\
\hline 17 & 1 & 0 & 2.968527 & 1.587333 & -0.204573 \\
\hline 18 & 6 & 0 & -2.174422 & -1.162144 & 0.129944 \\
\hline 19 & 6 & 0 & -3.591008 & -1.464054 & 0.139215 \\
\hline 20 & 6 & 0 & -4.103814 & -2.696164 & 0.284062 \\
\hline 21 & 1 & 0 & -5.174302 & -2.866426 & 0.288038 \\
\hline 22 & 1 & 0 & -3.460041 & -3.564552 & 0.399687 \\
\hline 23 & 1 & 0 & -1.496624 & -2.000586 & 0.278885 \\
\hline 24 & 1 & 0 & -4.277694 & -0.625719 & 0.021012 \\
\hline
\end{tabular}




$\begin{array}{rrrrrr}25 & 6 & 0 & -1.217676 & 2.570489 & -0.095188 \\ 26 & 1 & 0 & -1.347265 & 3.643870 & -0.129701 \\ 27 & 6 & 0 & -0.056286 & 1.897127 & -0.062476 \\ 28 & 1 & 0 & 0.901303 & 2.400308 & -0.087275 \\ 29 & 16 & 0 & -2.642444 & 1.542923 & -0.119138 \\ -\end{array}$

\section{Iminium D16}

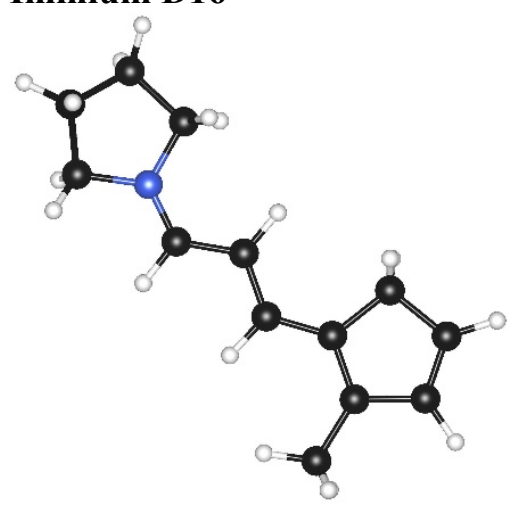

HF $(M 062 X / 6-31+G(d, p))=-560.4807685$ Hartrees

Imaginary Frequencies: none found

Zero-point correction $=0.285113$ (Hartree/Particle)

Thermal correction $=0.243995$ Hartrees

\section{Coordinates from last standard orientation:}

\begin{tabular}{|c|c|c|c|c|c|}
\hline \multirow{2}{*}{$\begin{array}{l}\text { Center } \\
\text { Number }\end{array}$} & \multirow{2}{*}{$\begin{array}{l}\text { Atomic } \\
\text { Number }\end{array}$} & \multirow{2}{*}{$\begin{array}{c}\text { Atomic } \\
\text { Type }\end{array}$} & \multicolumn{3}{|c|}{ Coordinates (Angstroms) } \\
\hline & & & $\mathrm{X}$ & Y & $\mathrm{z}$ \\
\hline 1 & 6 & 0 & -1.717095 & 0.429196 & -0.021872 \\
\hline 2 & 6 & 0 & -0.342112 & 0.639454 & -0.059523 \\
\hline 3 & 6 & 0 & 0.562277 & -0.458225 & -0.075570 \\
\hline 4 & 1 & 0 & 0.131964 & -1.454031 & -0.157742 \\
\hline 5 & 7 & 0 & 1.861905 & -0.450077 & -0.008842 \\
\hline 6 & 6 & 0 & 2.667160 & -1.700070 & -0.062392 \\
\hline 7 & 1 & 0 & 2.258924 & -2.366730 & -0.823707 \\
\hline 8 & 1 & 0 & 2.608818 & -2.186015 & 0.917625 \\
\hline 9 & 6 & 0 & 4.077164 & -1.196147 & -0.349851 \\
\hline 10 & 1 & 0 & 4.207686 & -1.027708 & -1.423401 \\
\hline 11 & 1 & 0 & 4.836514 & -1.905296 & -0.018283 \\
\hline 12 & 6 & 0 & 4.118772 & 0.130844 & 0.415012 \\
\hline 13 & 1 & 0 & 4.918083 & 0.794256 & 0.082754 \\
\hline 14 & 1 & 0 & 4.250001 & -0.051274 & 1.486018 \\
\hline 15 & 6 & 0 & 2.738931 & 0.734626 & 0.150346 \\
\hline 16 & 1 & 0 & 2.367417 & 1.356224 & 0.966951 \\
\hline 17 & 1 & 0 & 2.719172 & 1.305948 & -0.783549 \\
\hline 18 & 6 & 0 & -2.433715 & -0.822021 & 0.023850 \\
\hline 19 & 6 & 0 & -3.778781 & -0.903923 & 0.048627 \\
\hline 20 & 6 & 0 & -4.548182 & -2.178613 & 0.089844 \\
\hline 21 & 1 & 0 & -5.217818 & -2.244224 & -0.774511 \\
\hline 22 & 1 & 0 & -3.893102 & -3.051794 & 0.095956 \\
\hline 23 & 1 & 0 & -1.869512 & -1.751487 & 0.041870 \\
\hline 24 & 1 & 0 & -4.365164 & 0.014683 & 0.039430 \\
\hline 25 & 6 & 0 & -1.249778 & 2.748868 & -0.081187 \\
\hline
\end{tabular}




$\begin{array}{rrrrrr}26 & 1 & 0 & -1.380741 & 3.822763 & -0.111097 \\ 27 & 6 & 0 & -0.080904 & 2.092603 & -0.100157 \\ 28 & 1 & 0 & 0.891155 & 2.559525 & -0.160320 \\ 29 & 6 & 0 & -2.387947 & 1.775526 & -0.024451 \\ 30 & 1 & 0 & -3.005094 & 1.910801 & 0.873480 \\ 31 & 1 & 0 & -3.060828 & 1.879772 & -0.885753 \\ 32 & 1 & 0 & -5.185541 & -2.207582 & 0.980119 \\ -\end{array}$

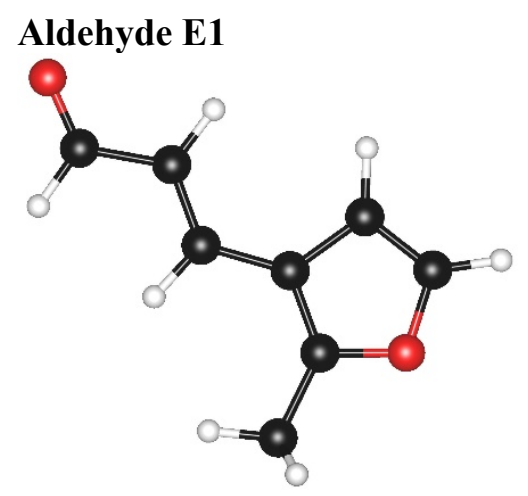

HF $(M 062 X / 6-31+G(d, p))=-459.8995532$ Hartrees

Imaginary Frequencies: none found

Zero-point correction $=0.142296$ (Hartree/Particle)

Thermal correction $=0.107131$ Hartrees

\section{Coordinates from last standard orientation:}

\begin{tabular}{|c|c|c|c|c|c|}
\hline \multirow{2}{*}{$\begin{array}{l}\text { Center } \\
\text { Number }\end{array}$} & \multirow{2}{*}{$\begin{array}{l}\text { Atomic } \\
\text { Number }\end{array}$} & \multirow{2}{*}{$\begin{array}{c}\text { Atomic } \\
\text { Type }\end{array}$} & \multicolumn{3}{|c|}{ Coordinates (Angstroms) } \\
\hline & & & $\mathrm{x}$ & $\mathrm{Y}$ & z \\
\hline 1 & 6 & 0 & 0.757427 & 0.479038 & 0.000182 \\
\hline 2 & 6 & 0 & 1.879217 & -0.265634 & 0.000242 \\
\hline 3 & 6 & 0 & 3.190157 & 0.391113 & 0.000018 \\
\hline 4 & 1 & 0 & 3.160620 & 1.502534 & 0.000196 \\
\hline 5 & 1 & 0 & 1.854113 & -1.351415 & 0.000341 \\
\hline 6 & 1 & 0 & 0.844920 & 1.564908 & 0.000153 \\
\hline 7 & 6 & 0 & -0.585896 & -0.033882 & 0.000150 \\
\hline 8 & 6 & 0 & -1.811344 & 0.587159 & -0.000172 \\
\hline 9 & 6 & 0 & -2.075144 & -1.632149 & -0.000056 \\
\hline 10 & 1 & 0 & -2.366082 & -2.671372 & -0.000074 \\
\hline 11 & 6 & 0 & -2.095239 & 2.056040 & 0.000046 \\
\hline 12 & 1 & 0 & -2.671399 & 2.338803 & 0.885890 \\
\hline 13 & 1 & 0 & -1.176452 & 2.645768 & -0.005341 \\
\hline 14 & 6 & 0 & -2.779071 & -0.467913 & -0.000346 \\
\hline 15 & 1 & 0 & -3.854673 & -0.366105 & -0.000679 \\
\hline 16 & 1 & 0 & -2.680602 & 2.337126 & -0.880234 \\
\hline 17 & 8 & 0 & -0.749684 & -1.388813 & 0.000298 \\
\hline 18 & 8 & 0 & 4.250798 & -0.196547 & -0.000377 \\
\hline
\end{tabular}




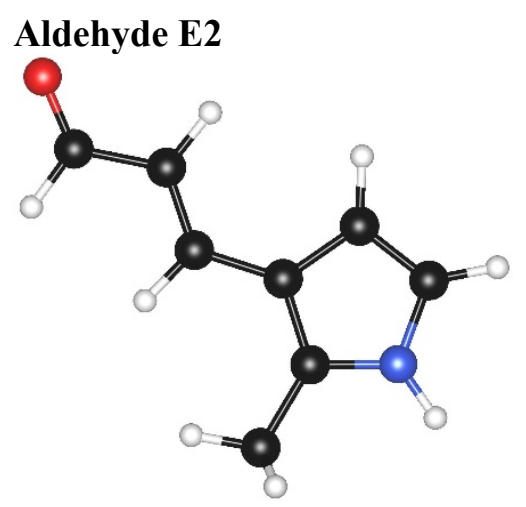

HF $(M 062 X / 6-31+G(d, p))=-440.0524551$ Hartrees

Imaginary Frequencies: none found

Zero-point correction $=0.154487$ (Hartree/Particle)

Thermal correction $=0.119864$ Hartrees

Coordinates from last standard orientation:

\begin{tabular}{|c|c|c|c|c|c|}
\hline \multirow{2}{*}{$\begin{array}{l}\text { Center } \\
\text { Number }\end{array}$} & \multirow{2}{*}{$\begin{array}{l}\text { Atomic } \\
\text { Number }\end{array}$} & \multirow{2}{*}{$\begin{array}{c}\text { Atomic } \\
\text { Type }\end{array}$} & \multicolumn{3}{|c|}{ Coordinates (Angstroms) } \\
\hline & & & $\mathrm{x}$ & Y & Z \\
\hline 1 & 6 & 0 & 0.766700 & 0.423655 & 0.000034 \\
\hline 2 & 6 & 0 & 1.907996 & -0.297987 & 0.000017 \\
\hline 3 & 6 & 0 & 3.205147 & 0.376714 & 0.000043 \\
\hline 4 & 1 & 0 & 3.156322 & 1.487253 & 0.000432 \\
\hline 5 & 1 & 0 & 1.932152 & -1.385188 & -0.000079 \\
\hline 6 & 1 & 0 & 0.857839 & 1.509478 & 0.000201 \\
\hline 7 & 6 & 0 & -0.580048 & -0.075399 & -0.000051 \\
\hline 8 & 6 & 0 & -1.788044 & 0.620376 & -0.000211 \\
\hline 9 & 6 & 0 & -2.231841 & -1.593432 & 0.000077 \\
\hline 10 & 1 & 0 & -2.669754 & -2.580565 & 0.000073 \\
\hline 11 & 6 & 0 & -1.976274 & 2.107023 & 0.000060 \\
\hline 12 & 1 & 0 & -2.529711 & 2.428712 & 0.887419 \\
\hline 13 & 1 & 0 & -1.024122 & 2.641650 & -0.008778 \\
\hline 14 & 6 & 0 & -2.824018 & -0.346691 & -0.000049 \\
\hline 15 & 1 & 0 & -3.886814 & -0.150321 & -0.000192 \\
\hline 16 & 1 & 0 & -2.544729 & 2.426711 & -0.878420 \\
\hline 17 & 8 & 0 & 4.277124 & -0.193160 & -0.000104 \\
\hline 18 & 7 & 0 & -0.883114 & -1.421716 & 0.000082 \\
\hline 19 & 1 & 0 & -0.204084 & -2.165991 & 0.000082 \\
\hline
\end{tabular}

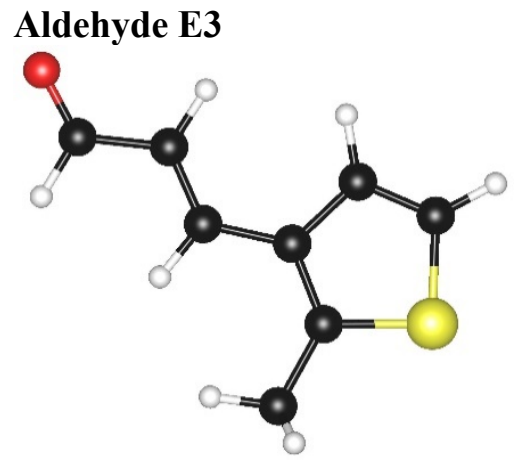


HF $(M 062 X / 6-31+G(d, p))=-782.865599$ Hartrees

Imaginary Frequencies: none found

Zero-point correction $=0.138198$ (Hartree/Particle)

Thermal correction $=0.101369$ Hartrees

Coordinates from last standard orientation:

\begin{tabular}{|c|c|c|c|c|c|}
\hline \multirow{2}{*}{$\begin{array}{l}\text { Center } \\
\text { Number }\end{array}$} & \multirow{2}{*}{$\begin{array}{l}\text { Atomic } \\
\text { Number }\end{array}$} & \multirow{2}{*}{$\begin{array}{c}\text { Atomic } \\
\text { Type }\end{array}$} & \multicolumn{3}{|c|}{ Coordinates (Angstroms) } \\
\hline & & & $\mathrm{X}$ & $\mathrm{Y}$ & Z \\
\hline 1 & 6 & 0 & 0.926549 & 0.528431 & 0.004386 \\
\hline 2 & 6 & 0 & 2.022446 & -0.254338 & -0.001684 \\
\hline 3 & 6 & 0 & 3.356248 & 0.354682 & 0.000700 \\
\hline 4 & 1 & 0 & 3.366117 & 1.466059 & 0.006957 \\
\hline 5 & 1 & 0 & 1.975204 & -1.340983 & -0.008455 \\
\hline 6 & 1 & 0 & 1.073545 & 1.609800 & 0.009277 \\
\hline 7 & 6 & 0 & -0.454458 & 0.101149 & 0.003767 \\
\hline 8 & 6 & 0 & -1.564857 & 0.924653 & 0.001802 \\
\hline 9 & 6 & 0 & -2.575878 & -1.174312 & -0.002870 \\
\hline 10 & 1 & 0 & -3.320406 & -1.958495 & -0.004191 \\
\hline 11 & 6 & 0 & -1.508831 & 2.428055 & -0.002066 \\
\hline 12 & 1 & 0 & -0.923428 & 2.810688 & 0.839358 \\
\hline 13 & 1 & 0 & -1.060345 & 2.808791 & -0.925064 \\
\hline 14 & 6 & 0 & -2.779074 & 0.177589 & -0.002331 \\
\hline 15 & 1 & 0 & -3.762245 & 0.634147 & -0.003921 \\
\hline 16 & 1 & 0 & -2.514497 & 2.845869 & 0.076027 \\
\hline 17 & 8 & 0 & 4.394619 & -0.271324 & -0.003606 \\
\hline 18 & 16 & 0 & -0.907735 & -1.576296 & 0.001789 \\
\hline
\end{tabular}

\section{Enol E4}

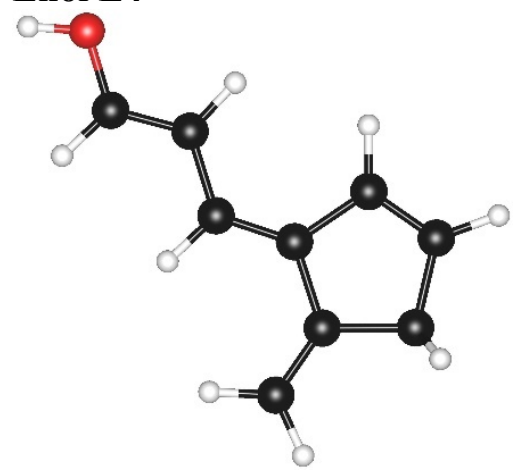

HF $(M 062 X / 6-31+G(d, p))=-423.9613425$ Hartrees

Imaginary Frequencies: none found

Zero-point correction $=0.164473$ (Hartree/Particle)

Thermal correction $=0.127394$ Hartrees

Coordinates from last standard orientation:

\begin{tabular}{cccccc} 
Center & Atomic & Atomic & \multicolumn{3}{c}{ Coordinates } \\
Number & Number & Type & X Angstroms) & Y \\
1 & 6 & 0 & -0.495803 & -0.672379 & 0.000000 \\
2 & 6 & 0 & 0.289802 & -1.887812 & 0.000000
\end{tabular}




$\begin{array}{rrrrrr}3 & 6 & 0 & -0.278206 & -3.101898 & 0.000000 \\ 4 & 1 & 0 & -1.359432 & -3.231789 & 0.000000 \\ 5 & 1 & 0 & 1.375079 & -1.832658 & 0.000000 \\ 6 & 1 & 0 & -1.578649 & -0.797508 & 0.000000 \\ 7 & 6 & 0 & 0.000000 & 0.584507 & 0.000000 \\ 8 & 6 & 0 & -0.792347 & 1.842581 & 0.000000 \\ 9 & 6 & 0 & 1.412743 & 0.987475 & 0.000000 \\ 10 & 6 & 0 & 1.534225 & 2.323023 & 0.000000 \\ 11 & 1 & 0 & 2.241479 & 0.288904 & 0.000000 \\ 12 & 1 & 0 & 2.473112 & 2.865432 & 0.000000 \\ 13 & 6 & 0 & -2.122602 & 1.966041 & 0.000000 \\ 14 & 1 & 0 & -2.595308 & 2.943513 & 0.000000 \\ 15 & 1 & 0 & -2.779518 & 1.101496 & 0.000000 \\ 16 & 6 & 0 & 0.190523 & 3.006337 & 0.000000 \\ 17 & 1 & 0 & 0.057711 & 3.645802 & 0.881276 \\ 18 & 1 & 0 & 0.057711 & 3.645802 & -0.881276 \\ 19 & 8 & 0 & 0.471436 & -4.237600 & 0.000000 \\ 20 & 1 & 0 & -0.093685 & -5.015454 & 0.000000 \\ ---------------\end{array}$

\section{Enol E5}

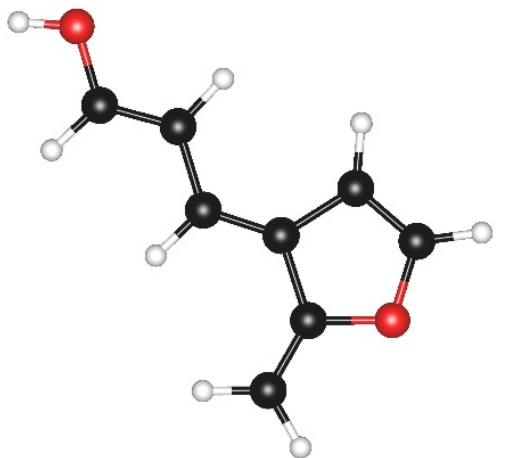

HF $(M 062 X / 6-31+G(d, p))=-459.8632432$ Hartrees

Imaginary Frequencies: none found

Zero-point correction $=0.142086$ (Hartree/Particle)

Thermal correction $=0.107406$ Hartrees

\section{Coordinates from last standard orientation:}

\begin{tabular}{|c|c|c|c|c|c|}
\hline \multirow{2}{*}{$\begin{array}{l}\text { Center } \\
\text { Number }\end{array}$} & \multirow{2}{*}{$\begin{array}{l}\text { Atomic } \\
\text { Number }\end{array}$} & \multirow{2}{*}{$\begin{array}{c}\text { Atomic } \\
\text { Type }\end{array}$} & \multicolumn{3}{|c|}{ Coordinates (Angstroms) } \\
\hline & & & $\mathrm{x}$ & Y & $\mathrm{Z}$ \\
\hline & & & -- & 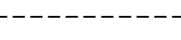 & -------- \\
\hline 1 & 6 & 0 & -0.600001 & 0.610946 & -0.000201 \\
\hline 2 & 6 & 0 & -1.834554 & -0.144729 & -0.000349 \\
\hline 3 & 6 & 0 & -3.037133 & 0.451659 & 0.000217 \\
\hline 4 & 1 & 0 & -3.155020 & 1.531409 & 0.000775 \\
\hline 5 & 1 & 0 & -1.761903 & -1.230844 & -0.000899 \\
\hline 6 & 1 & 0 & -0.655176 & 1.696268 & -0.000185 \\
\hline 7 & 6 & 0 & 0.623157 & 0.057730 & -0.000100 \\
\hline 8 & 6 & 0 & 1.969529 & 0.663833 & 0.000030 \\
\hline 9 & 6 & 0 & 2.107376 & -1.589701 & 0.000130 \\
\hline 10 & 1 & 0 & 2.362596 & -2.639932 & 0.000176 \\
\hline 11 & 6 & 0 & 2.285502 & 1.969956 & -0.000039 \\
\hline 12 & 1 & 0 & 3.321318 & 2.289499 & 0.000098 \\
\hline 13 & 1 & 0 & 1.523122 & 2.741514 & -0.000229 \\
\hline
\end{tabular}




\begin{tabular}{|c|c|c|c|c|c|}
\hline 14 & 6 & 0 & 2.872117 & -0.490595 & 0.000180 \\
\hline 15 & 1 & 0 & 3.951270 & -0.456522 & 0.000298 \\
\hline 16 & 8 & 0 & 0.766127 & -1.325243 & -0.000058 \\
\hline 17 & 8 & 0 & -4.239556 & -0.171850 & 0.000232 \\
\hline 18 & 1 & 0 & -4.114733 & -1.129244 & -0.000629 \\
\hline
\end{tabular}

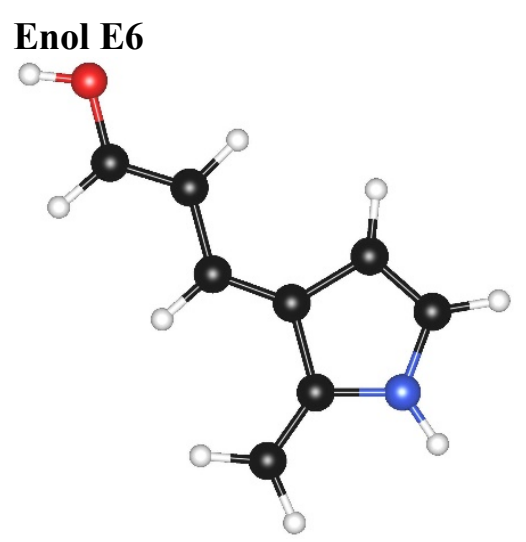

HF $(M 062 X / 6-31+G(d, p))=-440.0057536$ Hartrees

Imaginary Frequencies: none found

Zero-point correction $=0.154170$ (Hartree/Particle)

Thermal correction $=0.119075$ Hartrees

\section{Coordinates from last standard orientation:}

\begin{tabular}{|c|c|c|c|c|c|}
\hline \multirow{2}{*}{$\begin{array}{l}\text { Center } \\
\text { Number }\end{array}$} & \multirow{2}{*}{$\begin{array}{l}\text { Atomic } \\
\text { Number }\end{array}$} & \multirow{2}{*}{$\begin{array}{c}\text { Atomic } \\
\text { Type }\end{array}$} & \multicolumn{3}{|c|}{ Coordinates (Angstroms) } \\
\hline & & & $x$ & Y & z \\
\hline & & & & & \\
\hline 1 & 6 & 0 & -0.610640 & 0.578955 & -0.023314 \\
\hline 2 & 6 & 0 & -1.858502 & -0.152608 & 0.070809 \\
\hline 3 & 6 & 0 & -3.056304 & 0.429598 & -0.099515 \\
\hline 4 & 1 & 0 & -3.162545 & 1.482863 & -0.341697 \\
\hline 5 & 1 & 0 & -1.824113 & -1.213285 & 0.322858 \\
\hline 6 & 1 & 0 & -0.666976 & 1.658552 & -0.133800 \\
\hline 7 & 6 & 0 & 0.616551 & 0.021210 & 0.018802 \\
\hline 8 & 6 & 0 & 1.942188 & 0.694943 & -0.003443 \\
\hline 9 & 6 & 0 & 2.253453 & -1.559738 & -0.041401 \\
\hline 10 & 1 & 0 & 2.643959 & -2.568572 & -0.069135 \\
\hline 11 & 6 & 0 & 2.180910 & 2.016548 & 0.067434 \\
\hline 12 & 1 & 0 & 3.196208 & 2.396756 & 0.049875 \\
\hline 13 & 1 & 0 & 1.377800 & 2.740901 & 0.150916 \\
\hline 14 & 6 & 0 & 2.923287 & -0.391938 & -0.073716 \\
\hline 15 & 1 & 0 & 3.995609 & -0.266025 & -0.104359 \\
\hline 16 & 8 & 0 & -4.266651 & -0.174267 & 0.002308 \\
\hline 17 & 1 & 0 & -4.155069 & -1.104405 & 0.234657 \\
\hline 18 & 7 & 0 & 0.879156 & -1.361374 & 0.096398 \\
\hline 19 & 1 & 0 & 0.228594 & -2.024853 & -0.296514 \\
\hline
\end{tabular}




\section{Enol E7}

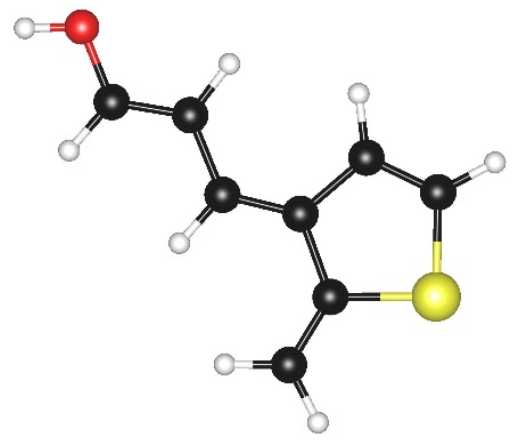

HF $(M 062 X / 6-31+G(d, p))=-782.8287946$ Hartrees

Imaginary Frequencies: none found

Zero-point correction $=0.138604$ (Hartree/Particle)

Thermal correction $=0.102369$ Hartrees

Coordinates from last standard orientation:

\begin{tabular}{|c|c|c|c|c|c|}
\hline \multirow{2}{*}{$\begin{array}{l}\text { Center } \\
\text { Number }\end{array}$} & \multirow{2}{*}{$\begin{array}{l}\text { Atomic } \\
\text { Number }\end{array}$} & \multirow{2}{*}{$\begin{array}{c}\text { Atomic } \\
\text { Type }\end{array}$} & \multicolumn{3}{|c|}{ Coordinates (Angstroms) } \\
\hline & & & $\mathrm{x}$ & Y & Z \\
\hline & & & & & 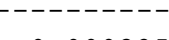 \\
\hline 1 & 6 & 0 & -0.776382 & 0.695943 & 0.000325 \\
\hline 2 & 6 & 0 & -1.987905 & -0.094670 & 0.000202 \\
\hline 3 & 6 & 0 & -3.206017 & 0.472105 & 0.000281 \\
\hline 4 & 1 & 0 & -3.346590 & 1.549220 & 0.000405 \\
\hline 5 & 1 & 0 & -1.901783 & -1.181183 & 0.000088 \\
\hline 6 & 1 & 0 & -0.897243 & 1.778124 & 0.001081 \\
\hline 7 & 6 & 0 & 0.479469 & 0.208589 & -0.000337 \\
\hline 8 & 6 & 0 & 1.741082 & 0.991546 & 0.000040 \\
\hline 9 & 6 & 0 & 2.561326 & -1.216600 & 0.000870 \\
\hline 10 & 1 & 0 & 3.242414 & -2.057930 & 0.001400 \\
\hline 11 & 6 & 0 & 1.838727 & 2.333243 & -0.000901 \\
\hline 12 & 1 & 0 & 2.811264 & 2.813168 & -0.000310 \\
\hline 13 & 1 & 0 & 0.967491 & 2.978709 & -0.002260 \\
\hline 14 & 6 & 0 & 2.890008 & 0.082417 & 0.001290 \\
\hline 15 & 1 & 0 & 3.911303 & 0.445293 & 0.002230 \\
\hline 16 & 8 & 0 & -4.392011 & -0.175997 & 0.000280 \\
\hline 17 & 1 & 0 & -4.250600 & -1.131271 & 0.000165 \\
\hline 18 & 16 & 0 & 0.834874 & -1.538850 & -0.000979 \\
\hline
\end{tabular}

\section{Aldehyde E8}

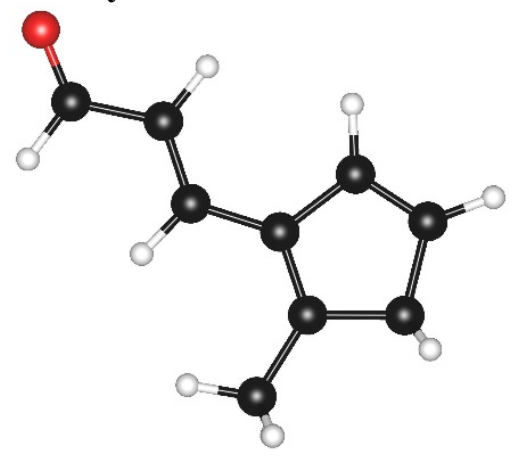


HF $(M 062 X / 6-31+G(d, p))=-423.9849446$ Hartrees

Imaginary Frequencies: none found

Zero-point correction $=0.164944$ (Hartree/Particle)

Thermal correction $=0.129505$ Hartrees

Coordinates from last standard orientation:

\begin{tabular}{|c|c|c|c|c|c|}
\hline \multirow{2}{*}{$\begin{array}{l}\text { Center } \\
\text { Number }\end{array}$} & \multirow{2}{*}{$\begin{array}{l}\text { Atomic } \\
\text { Number }\end{array}$} & \multirow{2}{*}{$\begin{array}{c}\text { Atomic } \\
\text { Type }\end{array}$} & \multicolumn{3}{|c|}{ Coordinates (Angstroms) } \\
\hline & & & $\mathrm{x}$ & $\mathrm{Y}$ & Z \\
\hline 1 & 6 & 0 & -0.783936 & 0.448481 & -0.000704 \\
\hline 2 & 6 & 0 & -1.911605 & -0.292869 & 0.000066 \\
\hline 3 & 6 & 0 & -3.224794 & 0.354874 & -0.000212 \\
\hline 4 & 1 & 0 & -3.200271 & 1.466582 & -0.001543 \\
\hline 5 & 1 & 0 & -1.898095 & -1.380196 & 0.001020 \\
\hline 6 & 1 & 0 & -0.894318 & 1.533762 & -0.001640 \\
\hline 7 & 6 & 0 & 0.562284 & -0.070200 & -0.000395 \\
\hline 8 & 6 & 0 & 1.719611 & 0.652694 & -0.000061 \\
\hline 9 & 6 & 0 & 2.401169 & -1.545595 & 0.000111 \\
\hline 10 & 1 & 0 & 3.001699 & -2.447050 & 0.000162 \\
\hline 11 & 6 & 0 & 1.898859 & 2.137522 & 0.000340 \\
\hline 12 & 1 & 0 & 2.471941 & 2.451439 & -0.878529 \\
\hline 13 & 1 & 0 & 0.949439 & 2.675010 & -0.003794 \\
\hline 14 & 6 & 0 & 2.854222 & -0.277537 & 0.000309 \\
\hline 15 & 1 & 0 & 3.892817 & 0.035867 & 0.000602 \\
\hline 16 & 6 & 0 & 0.900997 & -1.538314 & -0.000290 \\
\hline 17 & 1 & 0 & 0.493266 & -2.051522 & -0.881488 \\
\hline 18 & 1 & 0 & 0.492892 & -2.051432 & 0.880794 \\
\hline 19 & 1 & 0 & 2.464499 & 2.451871 & 0.883906 \\
\hline 20 & 8 & 0 & -4.284339 & -0.237333 & 0.000690 \\
\hline
\end{tabular}

\section{Iminium E9}

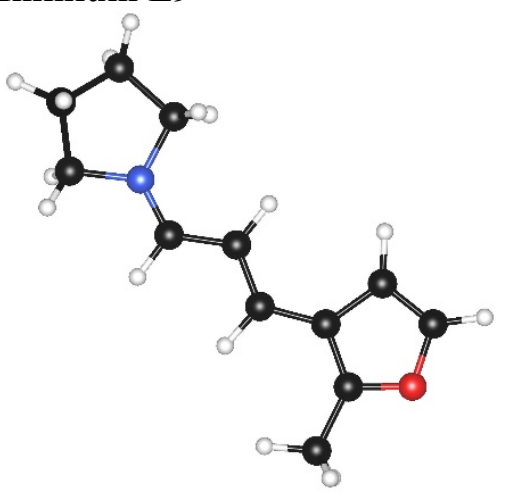

HF $(M 062 X / 6-31+G(d, p))=-596.4064371$ Hartrees

Imaginary Frequencies: none found

Zero-point correction $=0.262403$ (Hartree/Particle)

Thermal correction $=0.221077$ Hartrees

Coordinates from last standard orientation:

\begin{tabular}{|c|c|c|c|}
\hline Center & Atomic & Atomic & Coordinates (Angstroms) \\
\hline Number & Number & Type & $\mathrm{X}$ \\
\hline
\end{tabular}




\begin{tabular}{|c|c|c|c|c|c|}
\hline 1 & 6 & 0 & 1.067826 & 0.654190 & 0.032542 \\
\hline 2 & 6 & 0 & -0.152866 & 0.026918 & 0.040890 \\
\hline 3 & 6 & 0 & -1.327905 & 0.810419 & 0.067953 \\
\hline 4 & 1 & 0 & -1.236381 & 1.896558 & 0.079228 \\
\hline 5 & 7 & 0 & -2.545869 & 0.346055 & 0.076087 \\
\hline 6 & 6 & 0 & -3.758645 & 1.197117 & 0.079817 \\
\hline 7 & 1 & 0 & -3.598347 & 2.070909 & -0.554361 \\
\hline 8 & 1 & 0 & -3.951912 & 1.524111 & 1.107571 \\
\hline 9 & 6 & 0 & -4.843555 & 0.243520 & -0.412981 \\
\hline 10 & 1 & 0 & -4.825364 & 0.185758 & -1.505832 \\
\hline 11 & 1 & 0 & -5.838823 & 0.562574 & -0.101462 \\
\hline 12 & 6 & 0 & -4.419460 & -1.093802 & 0.205218 \\
\hline 13 & 1 & 0 & -4.874971 & -1.956051 & -0.282923 \\
\hline 14 & 1 & 0 & -4.686831 & -1.122361 & 1.265827 \\
\hline 15 & 6 & 0 & -2.896776 & -1.091897 & 0.048913 \\
\hline 16 & 1 & 0 & -2.371843 & -1.615112 & 0.851841 \\
\hline 17 & 1 & 0 & -2.578526 & -1.505002 & -0.914828 \\
\hline 18 & 1 & 0 & -0.209130 & -1.056038 & 0.021419 \\
\hline 19 & 1 & 0 & 1.108211 & 1.741835 & 0.051912 \\
\hline 20 & 6 & 0 & 2.313776 & 0.000439 & 0.000831 \\
\hline 21 & 6 & 0 & 3.615183 & 0.486863 & -0.010463 \\
\hline 22 & 6 & 0 & 3.616160 & -1.743616 & -0.055159 \\
\hline 23 & 1 & 0 & 3.800517 & -2.807780 & -0.079301 \\
\hline 24 & 6 & 0 & 4.060073 & 1.911360 & 0.010020 \\
\hline 25 & 1 & 0 & 4.651299 & 2.135352 & -0.882142 \\
\hline 26 & 1 & 0 & 3.220958 & 2.607947 & 0.045912 \\
\hline 27 & 6 & 0 & 4.450553 & -0.660083 & -0.046819 \\
\hline 28 & 1 & 0 & 5.530330 & -0.681981 & -0.064474 \\
\hline 29 & 1 & 0 & 4.694615 & 2.096558 & 0.881095 \\
\hline 30 & 8 & 0 & 2.330136 & -1.368530 & -0.027084 \\
\hline
\end{tabular}

\section{Iminium E10}

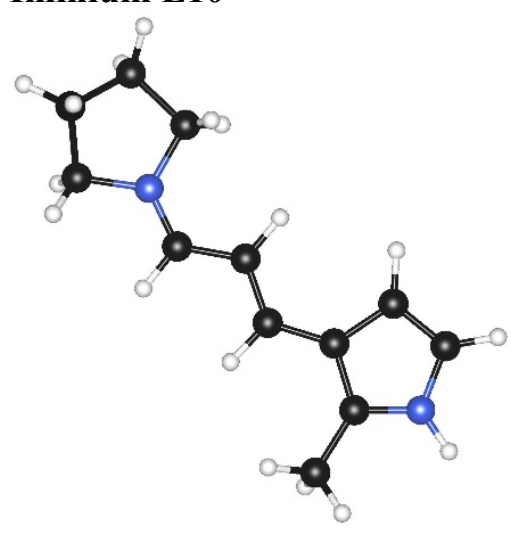

HF $(M 062 X / 6-31+G(d, p))=-576.5614715$ Hartrees

Imaginary Frequencies: none found

Zero-point correction $=0.275266$ (Hartree/Particle $)$

Thermal correction $=0.234156$ Hartrees

Coordinates from last standard orientation:

Center Atomic Atomic Coordinates (Angstroms)




\begin{tabular}{|c|c|c|c|c|c|}
\hline Number & Number & Type & $\mathrm{x}$ & $\mathrm{Y}$ & Z \\
\hline 1 & 6 & 0 & 1.063741 & 0.531954 & 0.022623 \\
\hline 2 & 6 & 0 & -0.179461 & -0.073439 & 0.035485 \\
\hline 3 & 6 & 0 & -1.328448 & 0.735181 & 0.059310 \\
\hline 4 & 1 & 0 & -1.203342 & 1.817893 & 0.064651 \\
\hline 5 & 7 & 0 & -2.566558 & 0.312474 & 0.071735 \\
\hline 6 & 6 & 0 & -3.746649 & 1.205778 & 0.072953 \\
\hline 7 & 1 & 0 & -3.560518 & 2.066517 & -0.572170 \\
\hline 8 & 1 & 0 & -3.926611 & 1.552476 & 1.096807 \\
\hline 9 & 6 & 0 & -4.866957 & 0.287251 & -0.407804 \\
\hline 10 & 1 & 0 & -4.856845 & 0.222767 & -1.500395 \\
\hline 11 & 1 & 0 & -5.849282 & 0.641062 & -0.092622 \\
\hline 12 & 6 & 0 & -4.485762 & -1.061168 & 0.214579 \\
\hline 13 & 1 & 0 & -4.972166 & -1.909584 & -0.268081 \\
\hline 14 & 1 & 0 & -4.749920 & -1.075860 & 1.276291 \\
\hline 15 & 6 & 0 & -2.963899 & -1.109672 & 0.053650 \\
\hline 16 & 1 & 0 & -2.457301 & -1.646752 & 0.860098 \\
\hline 17 & 1 & 0 & -2.663773 & -1.542578 & -0.907950 \\
\hline 18 & 1 & 0 & -0.286133 & -1.153296 & 0.024581 \\
\hline 19 & 1 & 0 & 1.092279 & 1.621442 & 0.038004 \\
\hline 20 & 6 & 0 & 2.322472 & -0.076296 & -0.006220 \\
\hline 21 & 6 & 0 & 3.591016 & 0.553678 & -0.007728 \\
\hline 22 & 6 & 0 & 3.877646 & -1.680240 & -0.058502 \\
\hline 23 & 1 & 0 & 4.267890 & -2.687957 & -0.086817 \\
\hline 24 & 6 & 0 & 3.840567 & 2.028901 & 0.029530 \\
\hline 25 & 1 & 0 & 4.909072 & 2.232833 & -0.051009 \\
\hline 26 & 1 & 0 & 3.339551 & 2.541873 & -0.796629 \\
\hline 27 & 6 & 0 & 4.552078 & -0.460558 & -0.040059 \\
\hline 28 & 1 & 0 & 5.624873 & -0.334234 & -0.049861 \\
\hline 29 & 1 & 0 & 3.486756 & 2.467104 & 0.967841 \\
\hline 30 & 7 & 0 & 2.552562 & -1.446284 & -0.037435 \\
\hline 31 & 1 & 0 & 1.845373 & -2.165257 & -0.049742 \\
\hline
\end{tabular}

\section{Iminium E11}

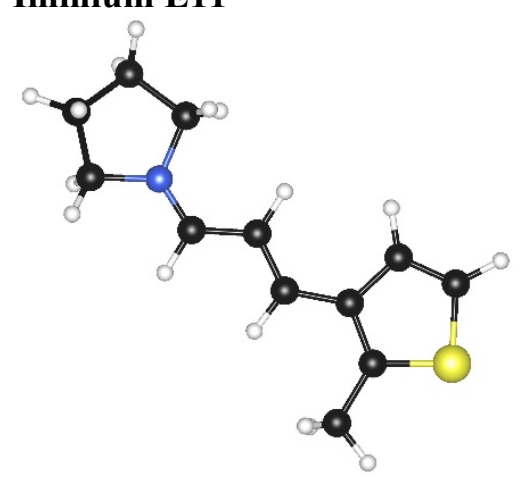

HF $($ M062X/6-31+G(d,p)) = -919.3709258 Hartrees

Imaginary Frequencies: none found

Zero-point correction $=0.258864$ (Hartree/Particle)

Thermal correction $=0.216555$ Hartrees

Coordinates from last standard orientation:

\begin{tabular}{|c|c|c|c|}
\hline Center & Atomic & Atomic & Coordinates (Angstroms) \\
\hline Number & Number & Type & $\mathrm{X}$ \\
\hline
\end{tabular}




\begin{tabular}{|c|c|c|c|c|c|}
\hline 1 & 6 & 0 & 0.828413 & 0.722743 & 0.030469 \\
\hline 2 & 6 & 0 & -0.381840 & 0.073524 & 0.045192 \\
\hline 3 & 6 & 0 & -1.570133 & 0.836593 & 0.066215 \\
\hline 4 & 1 & 0 & -1.494818 & 1.923990 & 0.070199 \\
\hline 5 & 7 & 0 & -2.780878 & 0.353823 & 0.076442 \\
\hline 6 & 6 & 0 & -4.006571 & 1.186368 & 0.073891 \\
\hline 7 & 1 & 0 & -3.857996 & 2.060100 & -0.563223 \\
\hline 8 & 1 & 0 & -4.207531 & 1.514082 & 1.099906 \\
\hline 9 & 6 & 0 & -5.075321 & 0.214367 & -0.418196 \\
\hline 10 & 1 & 0 & -5.052519 & 0.151934 & -1.510710 \\
\hline 11 & 1 & 0 & -6.076392 & 0.519664 & -0.111547 \\
\hline 12 & 6 & 0 & -4.633374 & -1.113586 & 0.207633 \\
\hline 13 & 1 & 0 & -5.073925 & -1.984972 & -0.278008 \\
\hline 14 & 1 & 0 & -4.903924 & -1.141303 & 1.267437 \\
\hline 15 & 6 & 0 & -3.110138 & -1.089293 & 0.056733 \\
\hline 16 & 1 & 0 & -2.581253 & -1.600118 & 0.865157 \\
\hline 17 & 1 & 0 & -2.782501 & -1.503622 & -0.903357 \\
\hline 18 & 1 & 0 & -0.434289 & -1.010866 & 0.035257 \\
\hline 19 & 1 & 0 & 0.820470 & 1.813593 & 0.042148 \\
\hline 20 & 6 & 0 & 2.113556 & 0.132595 & 0.001951 \\
\hline 21 & 6 & 0 & 3.328102 & 0.827772 & -0.007210 \\
\hline 22 & 6 & 0 & 4.057398 & -1.378007 & -0.049961 \\
\hline 23 & 1 & 0 & 4.706523 & -2.243788 & -0.072335 \\
\hline 24 & 6 & 0 & 3.455068 & 2.323327 & 0.017167 \\
\hline 25 & 1 & 0 & 4.504400 & 2.615087 & -0.040565 \\
\hline 26 & 1 & 0 & 2.934512 & 2.783516 & -0.827822 \\
\hline 27 & 6 & 0 & 4.430984 & -0.055738 & -0.036778 \\
\hline 28 & 1 & 0 & 5.463943 & 0.270274 & -0.048274 \\
\hline 29 & 1 & 0 & 3.044001 & 2.741033 & 0.941357 \\
\hline 30 & 16 & 0 & 2.365037 & -1.591835 & -0.026460 \\
\hline
\end{tabular}

Trienamine E12

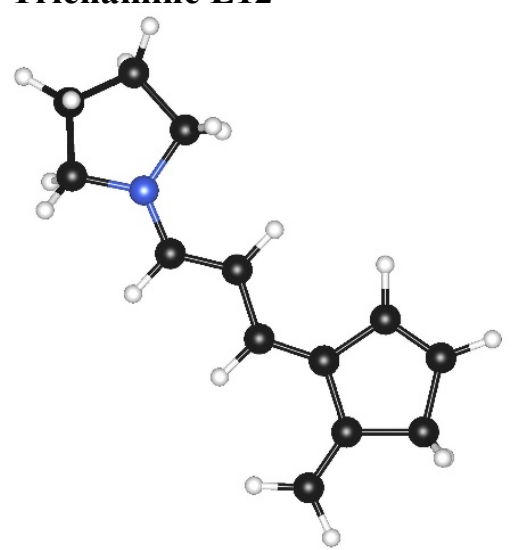

HF $(M 062 X / 6-31+G(d, p))=-560.0793104$ Hartrees

Imaginary Frequencies: none found

Zero-point correction $=0.271272$ (Hartree/Particle)

Thermal correction $=0.228995$ Hartrees

Coordinates from last standard orientation:

Center Atomic Atomic Coordinates (Angstroms)




\begin{tabular}{|c|c|c|c|c|c|}
\hline Number & Number & Type & $\mathrm{x}$ & $\mathrm{Y}$ & Z \\
\hline 1 & 6 & 0 & -1.136738 & 0.696726 & -0.029104 \\
\hline 2 & 6 & 0 & 0.167393 & 0.076363 & -0.037190 \\
\hline 3 & 6 & 0 & 1.308037 & 0.815608 & -0.063138 \\
\hline 4 & 1 & 0 & 1.239352 & 1.902977 & -0.079077 \\
\hline 5 & 7 & 0 & 2.577405 & 0.335833 & -0.064900 \\
\hline 6 & 6 & 0 & 3.770153 & 1.172923 & -0.077968 \\
\hline 7 & 1 & 0 & 3.645493 & 2.033808 & 0.587092 \\
\hline 8 & 1 & 0 & 3.983481 & 1.548100 & -1.090637 \\
\hline 9 & 6 & 0 & 4.862919 & 0.210121 & 0.390120 \\
\hline 10 & 1 & 0 & 4.862953 & 0.156962 & 1.484076 \\
\hline 11 & 1 & 0 & 5.859418 & 0.510124 & 0.058753 \\
\hline 12 & 6 & 0 & 4.405446 & -1.129324 & -0.201819 \\
\hline 13 & 1 & 0 & 4.853693 & -1.994412 & 0.291185 \\
\hline 14 & 1 & 0 & 4.663924 & -1.172776 & -1.265244 \\
\hline 15 & 6 & 0 & 2.881134 & -1.085339 & -0.040540 \\
\hline 16 & 1 & 0 & 2.356355 & -1.608304 & -0.850464 \\
\hline 17 & 1 & 0 & 2.550140 & -1.527861 & 0.911752 \\
\hline 18 & 1 & 0 & 0.225229 & -1.008859 & -0.018606 \\
\hline 19 & 1 & 0 & -1.155075 & 1.787629 & -0.047696 \\
\hline 20 & 6 & 0 & -2.312790 & 0.040429 & -0.001203 \\
\hline 21 & 6 & 0 & -3.661898 & 0.651546 & 0.006906 \\
\hline 22 & 6 & 0 & -3.972939 & -1.649129 & 0.052101 \\
\hline 23 & 1 & 0 & -4.449479 & -2.623367 & 0.076834 \\
\hline 24 & 6 & 0 & -3.999286 & 1.951910 & -0.011998 \\
\hline 25 & 1 & 0 & -5.040794 & 2.256152 & -0.002774 \\
\hline 26 & 1 & 0 & -3.253020 & 2.739607 & -0.038059 \\
\hline 27 & 6 & 0 & -4.614287 & -0.473171 & 0.040408 \\
\hline 28 & 1 & 0 & -5.690105 & -0.333774 & 0.053256 \\
\hline 29 & 6 & 0 & -2.475179 & -1.469507 & 0.027641 \\
\hline 30 & 1 & 0 & -2.000185 & -1.920410 & 0.910394 \\
\hline 31 & 1 & 0 & -2.025006 & -1.951372 & -0.851777 \\
\hline
\end{tabular}

\section{Fulvene trienaine E12'}

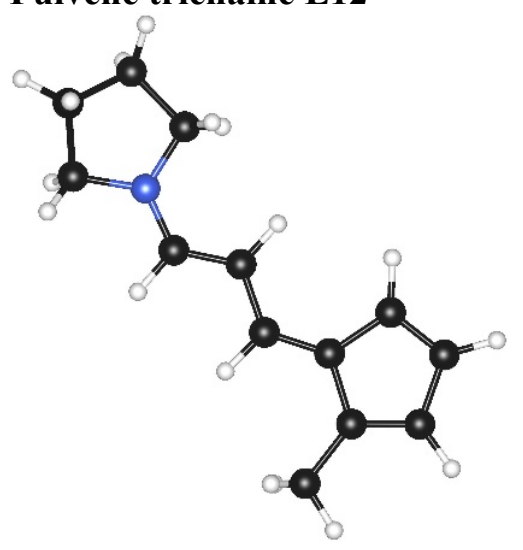

HF $(M 062 X / 6-31+G(d, p))=-560.0912156$ Hartrees

Imaginary Frequencies: none found Zero-point correction $=0.271814$ (Hartree/Particle) Thermal correction $=0.230197$ Hartrees

Coordinates from last standard orientation: 


\begin{tabular}{|c|c|c|c|c|c|}
\hline \multirow{2}{*}{$\begin{array}{l}\text { Center } \\
\text { Number }\end{array}$} & \multirow{2}{*}{$\begin{array}{l}\text { Atomic } \\
\text { Number }\end{array}$} & \multirow{2}{*}{$\begin{array}{c}\text { Atomic } \\
\text { Type }\end{array}$} & \multicolumn{3}{|c|}{ Coordinates (Angstroms) } \\
\hline & & & $\mathrm{x}$ & Y & Z \\
\hline 1 & 6 & 0 & -1.132359 & -0.547378 & 0.028635 \\
\hline 2 & 6 & 0 & 0.172335 & 0.040713 & 0.037266 \\
\hline 3 & 6 & 0 & 1.289509 & -0.742835 & 0.061532 \\
\hline 4 & 1 & 0 & 1.177812 & -1.826813 & 0.073492 \\
\hline 5 & 7 & 0 & 2.568307 & -0.313723 & 0.067482 \\
\hline 6 & 6 & 0 & 3.730157 & -1.198531 & 0.066895 \\
\hline 7 & 1 & 0 & 3.566748 & -2.047108 & -0.604591 \\
\hline 8 & 1 & 0 & 3.927779 & -1.587659 & 1.076379 \\
\hline 9 & 6 & 0 & 4.858202 & -0.275125 & -0.395569 \\
\hline 10 & 1 & 0 & 4.857839 & -0.210908 & -1.488841 \\
\hline 11 & 1 & 0 & 5.842174 & -0.618672 & -0.069828 \\
\hline 12 & 6 & 0 & 4.456555 & 1.075167 & 0.211609 \\
\hline 13 & 1 & 0 & 4.939936 & 1.926112 & -0.272227 \\
\hline 14 & 1 & 0 & 4.715431 & 1.096672 & 1.275466 \\
\hline 15 & 6 & 0 & 2.932965 & 1.097340 & 0.047589 \\
\hline 16 & 1 & 0 & 2.426614 & 1.636964 & 0.856760 \\
\hline 17 & 1 & 0 & 2.624160 & 1.551219 & -0.905589 \\
\hline 18 & 1 & 0 & 0.261489 & 1.122643 & 0.020912 \\
\hline 19 & 1 & 0 & -1.165092 & -1.639267 & 0.048779 \\
\hline 20 & 6 & 0 & -2.324033 & 0.111284 & -0.001140 \\
\hline 21 & 6 & 0 & -3.644396 & -0.533426 & -0.007600 \\
\hline 22 & 6 & 0 & -2.559540 & 1.554259 & -0.035497 \\
\hline 23 & 6 & 0 & -3.903212 & 1.753678 & -0.060509 \\
\hline 24 & 1 & 0 & -1.791888 & 2.317618 & -0.041899 \\
\hline 25 & 1 & 0 & -4.407576 & 2.712287 & -0.090149 \\
\hline 26 & 6 & 0 & -3.877273 & -2.012959 & 0.023949 \\
\hline 27 & 1 & 0 & -4.948221 & -2.230344 & 0.001328 \\
\hline 28 & 1 & 0 & -3.416797 & -2.514809 & -0.835018 \\
\hline 29 & 6 & 0 & -4.577702 & 0.455462 & -0.042663 \\
\hline 30 & 1 & 0 & -5.652365 & 0.311090 & -0.055304 \\
\hline 31 & 1 & 0 & -3.463442 & -2.468855 & 0.930980 \\
\hline
\end{tabular}

\section{Trienamine E13}

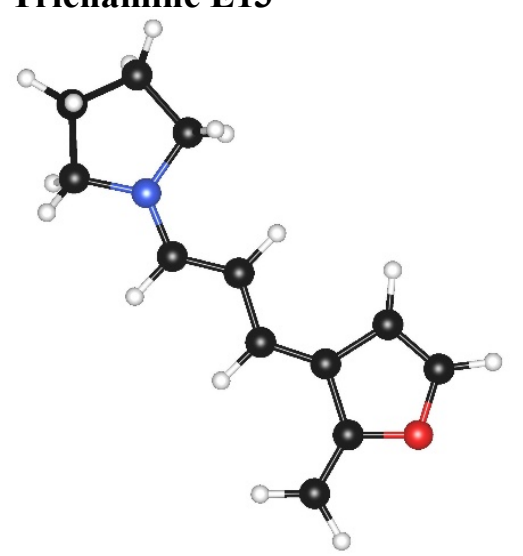

HF $(M 062 X / 6-31+G(d, p))=-595.9781919$ Hartrees

Imaginary Frequencies: none found Zero-point correction $=0.247817$ (Hartree/Particle) Thermal correction $=0.206159$ Hartrees

Coordinates from last standard orientation: 


\begin{tabular}{|c|c|c|c|c|c|}
\hline \multirow{2}{*}{$\begin{array}{l}\text { Center } \\
\text { Number }\end{array}$} & \multirow{2}{*}{$\begin{array}{l}\text { Atomic } \\
\text { Number }\end{array}$} & \multirow{2}{*}{$\begin{array}{c}\text { Atomic } \\
\text { Type }\end{array}$} & \multicolumn{3}{|c|}{ Coordinates (Angstroms) } \\
\hline & & & $\mathrm{x}$ & $\mathrm{Y}$ & z \\
\hline 1 & 6 & 0 & -1.150482 & 0.733600 & -0.031192 \\
\hline 2 & 6 & 0 & 0.145015 & 0.104943 & -0.038036 \\
\hline 3 & 6 & 0 & 1.289715 & 0.838323 & -0.062832 \\
\hline 4 & 1 & 0 & 1.230847 & 1.926266 & -0.077863 \\
\hline 5 & 7 & 0 & 2.554690 & 0.347002 & -0.064257 \\
\hline 6 & 6 & 0 & 3.754793 & 1.172983 & -0.073757 \\
\hline 7 & 1 & 0 & 3.638317 & 2.032315 & 0.594972 \\
\hline 8 & 1 & 0 & 3.971803 & 1.550627 & -1.084843 \\
\hline 9 & 6 & 0 & 4.838766 & 0.198248 & 0.390306 \\
\hline 10 & 1 & 0 & 4.838701 & 0.141168 & 1.484075 \\
\hline 11 & 1 & 0 & 5.837897 & 0.490275 & 0.059652 \\
\hline 12 & 6 & 0 & 4.368385 & -1.134769 & -0.206064 \\
\hline 13 & 1 & 0 & 4.809116 & -2.005723 & 0.283374 \\
\hline 14 & 1 & 0 & 4.625508 & -1.176680 & -1.269901 \\
\hline 15 & 6 & 0 & 2.844746 & -1.077413 & -0.043074 \\
\hline 16 & 1 & 0 & 2.313894 & -1.593396 & -0.853213 \\
\hline 17 & 1 & 0 & 2.510529 & -1.518553 & 0.908490 \\
\hline 18 & 1 & 0 & 0.182098 & -0.979735 & -0.019119 \\
\hline 19 & 1 & 0 & -1.197529 & 1.820382 & -0.051851 \\
\hline 20 & 6 & 0 & -2.327768 & 0.082803 & -0.001624 \\
\hline 21 & 6 & 0 & -3.715626 & 0.575124 & 0.007986 \\
\hline 22 & 6 & 0 & -3.665614 & -1.682805 & 0.052640 \\
\hline 23 & 1 & 0 & -3.836389 & -2.750030 & 0.076356 \\
\hline 24 & 6 & 0 & -4.147459 & 1.850305 & -0.011207 \\
\hline 25 & 1 & 0 & -5.207588 & 2.075883 & -0.000920 \\
\hline 26 & 1 & 0 & -3.457409 & 2.686584 & -0.037509 \\
\hline 27 & 6 & 0 & -4.519508 & -0.649704 & 0.043728 \\
\hline 28 & 1 & 0 & -5.597817 & -0.704789 & 0.059733 \\
\hline 29 & 8 & 0 & -2.354323 & -1.311679 & 0.027139 \\
\hline
\end{tabular}

\section{Trienamine E14}

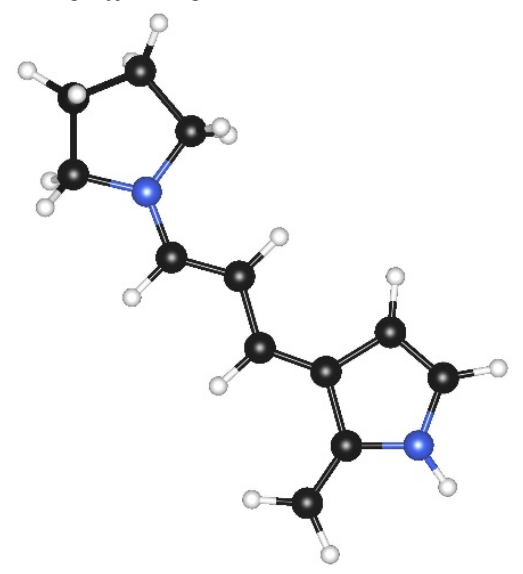

HF $(M 062 X / 6-31+G(d, p))=-576.1207353$ Hartrees

Imaginary Frequencies: none found Zero-point correction $=0.260177$ (Hartree/Particle)

Thermal correction $=0.218584$ Hartrees 
Coordinates from last standard orientation:

\begin{tabular}{|c|c|c|c|c|c|}
\hline \multirow{2}{*}{$\begin{array}{l}\text { Center } \\
\text { Number }\end{array}$} & \multirow{2}{*}{$\begin{array}{l}\text { Atomic } \\
\text { Number }\end{array}$} & \multirow{2}{*}{$\begin{array}{c}\text { Atomic } \\
\text { Type }\end{array}$} & \multicolumn{3}{|c|}{ Coordinates (Angstroms) } \\
\hline & & & $\mathrm{x}$ & Y & Z \\
\hline 1 & 6 & 0 & -1.147217 & 0.704635 & 0000538 \\
\hline 2 & 6 & 0 & 0.157289 & 0.087435 & -0.027876 \\
\hline 3 & 6 & 0 & 1.300043 & 0.816200 & 0.076473 \\
\hline 4 & 1 & 0 & 1.234240 & 1.896341 & 0.202694 \\
\hline 5 & 7 & 0 & 2.570531 & 0.332573 & 0.052214 \\
\hline 6 & 6 & 0 & 3.760030 & 1.172207 & 0.072709 \\
\hline 7 & 1 & 0 & 3.669969 & 1.948959 & 0.839082 \\
\hline 8 & 1 & 0 & 3.921750 & 1.664292 & -0.899426 \\
\hline 9 & 6 & 0 & 4.872941 & 0.165574 & 0.365570 \\
\hline 10 & 1 & 0 & 4.919365 & -0.028533 & 1.442382 \\
\hline 11 & 1 & 0 & 5.853775 & 0.512907 & 0.033718 \\
\hline 12 & 6 & 0 & 4.392616 & -1.087629 & -0.378211 \\
\hline 13 & 1 & 0 & 4.855253 & -2.007940 & -0.015540 \\
\hline 14 & 1 & 0 & 4.618226 & -0.993329 & -1.445737 \\
\hline 15 & 6 & 0 & 2.872512 & -1.071650 & -0.166665 \\
\hline 16 & 1 & 0 & 2.325279 & -1.455434 & -1.038617 \\
\hline 17 & 1 & 0 & 2.566831 & -1.666880 & 0.707262 \\
\hline 18 & 1 & 0 & 0.223198 & -0.989559 & -0.163161 \\
\hline 19 & 1 & 0 & -1.188341 & 1.791179 & 0.036667 \\
\hline 20 & 6 & 0 & -2.327818 & 0.048755 & -0.013549 \\
\hline 21 & 6 & 0 & -3.701586 & 0.607826 & -0.041564 \\
\hline 22 & 6 & 0 & -3.820803 & -1.659093 & 0.155788 \\
\hline 23 & 1 & 0 & -4.127834 & -2.692298 & 0.255218 \\
\hline 24 & 6 & 0 & -4.057402 & 1.896720 & -0.206231 \\
\hline 25 & 1 & 0 & -5.102533 & 2.185589 & -0.215440 \\
\hline 26 & 1 & 0 & -3.320302 & 2.681030 & -0.340567 \\
\hline 27 & 6 & 0 & -4.587068 & -0.551489 & 0.103963 \\
\hline 28 & 1 & 0 & -5.666641 & -0.516261 & 0.122864 \\
\hline 29 & 7 & 0 & -2.469768 & -1.358030 & 0.002327 \\
\hline 30 & 1 & 0 & -1.768801 & -1.928817 & 0.451153 \\
\hline
\end{tabular}

\section{Fulvene trienamine E14'}

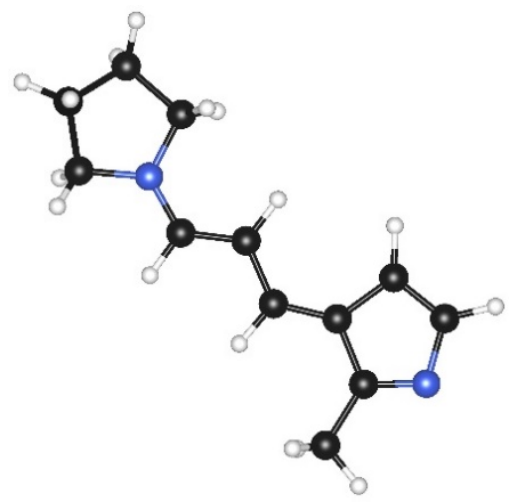

HF $(M 062 X / 6-31+G(d, p))=-576.138208$ Hartrees

Imaginary Frequencies: none found

Zero-point correction $=0.260572$ (Hartree/Particle)

Thermal correction $=0.218975$ Hartrees

Coordinates from last standard orientation: 


\begin{tabular}{|c|c|c|c|c|c|}
\hline \multirow{2}{*}{$\begin{array}{l}\text { Center } \\
\text { Number }\end{array}$} & \multirow{2}{*}{$\begin{array}{l}\text { Atomic } \\
\text { Number }\end{array}$} & \multirow{2}{*}{$\begin{array}{l}\text { Atomic } \\
\text { Type }\end{array}$} & \multicolumn{3}{|c|}{ Coordinates (Angstroms) } \\
\hline & & & $\mathrm{x}$ & Y & Z \\
\hline 1 & 6 & 0 & -1.124805 & -0.524237 & 0.028073 \\
\hline 2 & 6 & 0 & 0.175620 & 0.060634 & 0.036302 \\
\hline 3 & 6 & 0 & 1.290971 & -0.730905 & 0.062381 \\
\hline 4 & 1 & 0 & 1.171225 & -1.814051 & 0.075887 \\
\hline 5 & 7 & 0 & 2.567785 & -0.310860 & 0.068536 \\
\hline 6 & 6 & 0 & 3.726049 & -1.203421 & 0.071548 \\
\hline 7 & 1 & 0 & 3.555573 & -2.054312 & -0.594643 \\
\hline 8 & 1 & 0 & 3.919371 & -1.586134 & 1.083716 \\
\hline 9 & 6 & 0 & 4.858867 & -0.288677 & -0.395402 \\
\hline 10 & 1 & 0 & 4.857910 & -0.227913 & -1.488803 \\
\hline 11 & 1 & 0 & 5.840575 & -0.637988 & -0.069469 \\
\hline 12 & 6 & 0 & 4.467005 & 1.065802 & 0.208823 \\
\hline 13 & 1 & 0 & 4.955790 & 1.911993 & -0.277577 \\
\hline 14 & 1 & 0 & 4.726484 & 1.088455 & 1.272413 \\
\hline 15 & 6 & 0 & 2.943780 & 1.099153 & 0.044938 \\
\hline 16 & 1 & 0 & 2.440328 & 1.643503 & 0.852166 \\
\hline 17 & 1 & 0 & 2.638320 & 1.549885 & -0.910212 \\
\hline 18 & 1 & 0 & 0.265994 & 1.142392 & 0.018550 \\
\hline 19 & 1 & 0 & -1.163657 & -1.615865 & 0.048357 \\
\hline 20 & 6 & 0 & -2.315932 & 0.138575 & -0.001676 \\
\hline 21 & 6 & 0 & -3.639592 & -0.497214 & -0.008892 \\
\hline 22 & 6 & 0 & -2.608376 & 1.562736 & -0.036500 \\
\hline 23 & 6 & 0 & -3.962471 & 1.649657 & -0.059915 \\
\hline 24 & 1 & 0 & -1.897223 & 2.377327 & -0.043819 \\
\hline 25 & 1 & 0 & -4.573426 & 2.543143 & -0.088285 \\
\hline 26 & 6 & 0 & -3.910320 & -1.969375 & 0.024033 \\
\hline 27 & 1 & 0 & -4.988739 & -2.130269 & -0.013811 \\
\hline 28 & 1 & 0 & -3.449062 & -2.481907 & -0.827078 \\
\hline 29 & 7 & 0 & -4.594052 & 0.388297 & -0.042770 \\
\hline 30 & 1 & 0 & -3.520363 & -2.426689 & 0.939976 \\
\hline
\end{tabular}

\section{Trienamine E15}

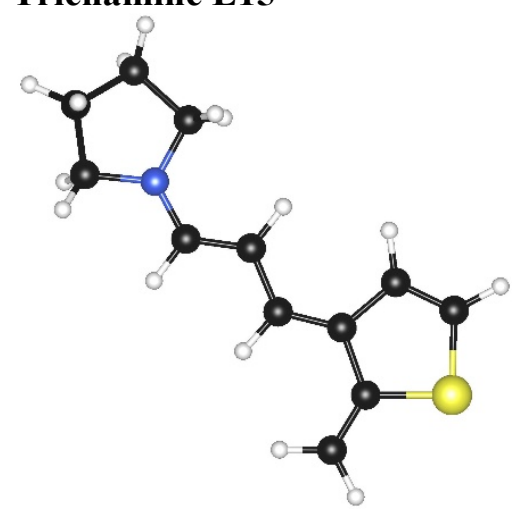

HF $(M 062 X / 6-31+G(d, p))=-918.9451571$ Hartrees Imaginary Frequencies: none found Zero-point correction $=0.244509$ (Hartree/Particle) Thermal correction $=0.201144$ Hartrees

Coordinates from last standard orientation: 


\begin{tabular}{|c|c|c|c|c|c|}
\hline \multirow{2}{*}{$\begin{array}{l}\text { Center } \\
\text { Number }\end{array}$} & \multirow{2}{*}{$\begin{array}{l}\text { Atomic } \\
\text { Number }\end{array}$} & \multirow{2}{*}{$\begin{array}{c}\text { Atomic } \\
\text { Type }\end{array}$} & \multicolumn{3}{|c|}{ Coordinates (Angstroms) } \\
\hline & & & $\mathrm{x}$ & $\mathrm{Y}$ & z \\
\hline 1 & 6 & 0 & -0.904449 & 0.848680 & -0.035707 \\
\hline 2 & 6 & 0 & 0.374340 & 0.191099 & -0.050000 \\
\hline 3 & 6 & 0 & 1.538101 & 0.897517 & -0.067011 \\
\hline 4 & 1 & 0 & 1.503511 & 1.986538 & -0.072611 \\
\hline 5 & 7 & 0 & 2.787721 & 0.376503 & -0.071522 \\
\hline 6 & 6 & 0 & 4.009690 & 1.171801 & -0.067592 \\
\hline 7 & 1 & 0 & 3.907548 & 2.033227 & 0.600365 \\
\hline 8 & 1 & 0 & 4.244949 & 1.543136 & -1.076453 \\
\hline 9 & 6 & 0 & 5.063311 & 0.168702 & 0.405691 \\
\hline 10 & 1 & 0 & 5.049996 & 0.108963 & 1.499206 \\
\hline 11 & 1 & 0 & 6.072905 & 0.436169 & 0.086552 \\
\hline 12 & 6 & 0 & 4.565848 & -1.150474 & -0.199578 \\
\hline 13 & 1 & 0 & 4.979063 & -2.033600 & 0.291784 \\
\hline 14 & 1 & 0 & 4.832449 & -1.196168 & -1.260854 \\
\hline 15 & 6 & 0 & 3.042588 & -1.055648 & -0.051400 \\
\hline 16 & 1 & 0 & 2.506793 & -1.556347 & -0.867592 \\
\hline 17 & 1 & 0 & 2.688440 & -1.488640 & 0.896372 \\
\hline 18 & 1 & 0 & 0.400287 & -0.895516 & -0.042328 \\
\hline 19 & 1 & 0 & -0.896851 & 1.938833 & -0.043648 \\
\hline 20 & 6 & 0 & -2.112719 & 0.244530 & -0.011227 \\
\hline 21 & 6 & 0 & -3.440987 & 0.897337 & 0.008059 \\
\hline 22 & 6 & 0 & -4.035899 & -1.383803 & 0.059527 \\
\hline 23 & 1 & 0 & -4.631724 & -2.287372 & 0.086978 \\
\hline 24 & 6 & 0 & -3.680938 & 2.223209 & -0.015189 \\
\hline 25 & 1 & 0 & -4.698562 & 2.597339 & 0.004997 \\
\hline 26 & 1 & 0 & -2.883016 & 2.956432 & -0.055702 \\
\hline 27 & 6 & 0 & -4.492259 & -0.122731 & 0.054293 \\
\hline 28 & 1 & 0 & -5.544707 & 0.136373 & 0.078043 \\
\hline 29 & 16 & 0 & -2.287805 & -1.531013 & 0.012272 \\
\hline
\end{tabular}

\section{Iminium E16}

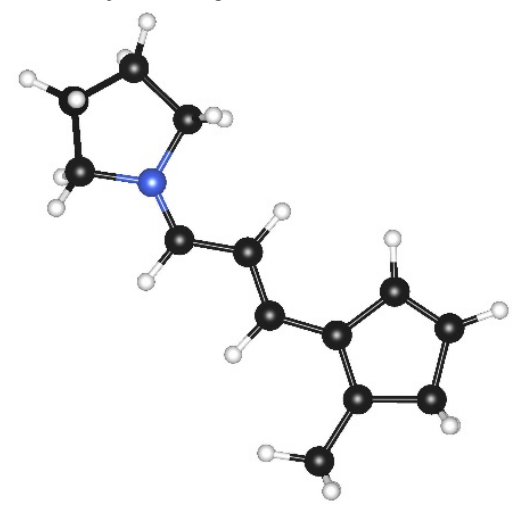

HF $(M 062 X / 6-31+G(d, p))=-560.4940946$ Hartrees

Imaginary Frequencies: none found

Zero-point correction $=0.285296$ (Hartree/Particle)

Thermal correction $=0.244124$ Hartrees

Coordinates from last standard orientation: 


\begin{tabular}{|c|c|c|c|c|c|}
\hline \multirow{2}{*}{$\begin{array}{l}\text { Center } \\
\text { Number }\end{array}$} & \multirow{2}{*}{$\begin{array}{l}\text { Atomic } \\
\text { Number }\end{array}$} & \multirow{2}{*}{$\begin{array}{c}\text { Atomic } \\
\text { Type }\end{array}$} & \multicolumn{3}{|c|}{ Coordinates (Angstroms) } \\
\hline & & & $\mathrm{x}$ & $\mathrm{Y}$ & Z \\
\hline 1 & 6 & 0 & 1.050474 & 0.600880 & 0.029048 \\
\hline 2 & 6 & 0 & -0.181101 & -0.017489 & 0.038623 \\
\hline 3 & 6 & 0 & -1.347808 & 0.772659 & 0.065486 \\
\hline 4 & 1 & 0 & -1.244281 & 1.857721 & 0.076372 \\
\hline 5 & 7 & 0 & -2.574357 & 0.324044 & 0.074431 \\
\hline 6 & 6 & 0 & -3.774617 & 1.191121 & 0.079290 \\
\hline 7 & 1 & 0 & -3.604868 & 2.061218 & -0.557618 \\
\hline 8 & 1 & 0 & -3.963140 & 1.524223 & 1.106063 \\
\hline 9 & 6 & 0 & -4.873454 & 0.251832 & -0.410438 \\
\hline 10 & 1 & 0 & -4.858669 & 0.193769 & -1.503345 \\
\hline 11 & 1 & 0 & -5.863919 & 0.583290 & -0.096478 \\
\hline 12 & 6 & 0 & -4.465252 & -1.091247 & 0.206107 \\
\hline 13 & 1 & 0 & -4.932726 & -1.947362 & -0.281606 \\
\hline 14 & 1 & 0 & -4.731213 & -1.116785 & 1.267176 \\
\hline 15 & 6 & 0 & -2.942703 & -1.107640 & 0.047772 \\
\hline 16 & 1 & 0 & -2.424758 & -1.638050 & 0.850893 \\
\hline 17 & 1 & 0 & -2.631318 & -1.526845 & -0.915908 \\
\hline 18 & 1 & 0 & -0.258488 & -1.099964 & 0.021048 \\
\hline 19 & 1 & 0 & 1.064196 & 1.690586 & 0.047219 \\
\hline 20 & 6 & 0 & 2.299293 & -0.054039 & -0.001204 \\
\hline 21 & 6 & 0 & 3.534538 & 0.569719 & -0.007357 \\
\hline 22 & 6 & 0 & 3.997449 & -1.672574 & -0.056040 \\
\hline 23 & 1 & 0 & 4.517506 & -2.622908 & -0.082144 \\
\hline 24 & 6 & 0 & 3.846733 & 2.026383 & 0.016253 \\
\hline 25 & 1 & 0 & 4.441554 & 2.291085 & -0.863977 \\
\hline 26 & 1 & 0 & 2.960065 & 2.660142 & 0.036065 \\
\hline 27 & 6 & 0 & 4.570681 & -0.448767 & -0.041525 \\
\hline 28 & 1 & 0 & 5.632666 & -0.233378 & -0.052921 \\
\hline 29 & 6 & 0 & 2.506428 & -1.545173 & -0.032106 \\
\hline 30 & 1 & 0 & 2.055414 & -2.012549 & -0.917759 \\
\hline 31 & 1 & 0 & 2.080605 & -2.045493 & 0.848001 \\
\hline 32 & 1 & 0 & 4.457908 & 2.259006 & 0.894444 \\
\hline
\end{tabular}

\section{Model A: N-Methyl Aldehyde}

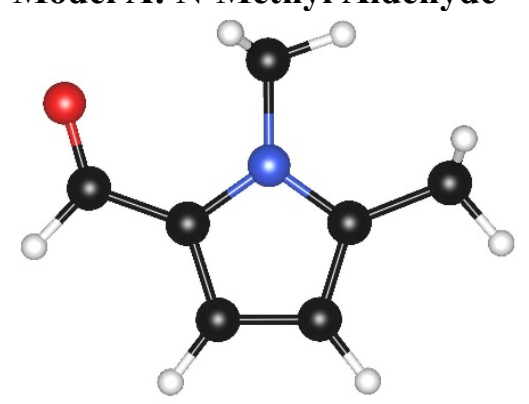

HF $(M 062 X / 6-31+G(d, p))=-401.9760183$ Hartrees

Imaginary Frequencies: none found

Zero-point correction $=0.149452$ (Hartree/Particle)

Thermal correction $=0.115336$ Hartrees

Coordinates from last standard orientation:

Center Atomic Atomic Coordinates (Angstroms)




\begin{tabular}{|c|c|c|c|c|c|}
\hline Number & Number & Type & $\mathrm{x}$ & $\mathrm{Y}$ & Z \\
\hline 1 & 6 & 0 & 1.431694 & 0.097266 & 0.000049 \\
\hline 2 & 6 & 0 & 1.331592 & 1.485019 & 0.000243 \\
\hline 3 & 6 & 0 & -0.035549 & 1.807896 & 0.000246 \\
\hline 4 & 6 & 0 & -0.742589 & 0.611924 & -0.000176 \\
\hline 5 & 7 & 0 & 0.173284 & -0.424027 & -0.000049 \\
\hline 6 & 1 & 0 & 2.170060 & 2.166626 & 0.000393 \\
\hline 7 & 1 & 0 & -0.481136 & 2.793490 & 0.000367 \\
\hline 8 & 6 & 0 & 2.662441 & -0.750927 & 0.000156 \\
\hline 9 & 1 & 0 & 2.716414 & -1.392257 & 0.886039 \\
\hline 10 & 1 & 0 & 2.717117 & -1.391562 & -0.886195 \\
\hline 11 & 1 & 0 & 3.540221 & -0.102981 & 0.000718 \\
\hline 12 & 6 & 0 & -2.179749 & 0.439658 & -0.000495 \\
\hline 13 & 1 & 0 & -2.737868 & 1.397026 & -0.000637 \\
\hline 14 & 8 & 0 & -2.773108 & -0.626205 & -0.000456 \\
\hline 15 & 6 & 0 & -0.166760 & -1.840676 & 0.000318 \\
\hline 16 & 1 & 0 & -0.753906 & -2.087761 & 0.885908 \\
\hline 17 & 1 & 0 & -0.761887 & -2.085904 & -0.880354 \\
\hline 18 & 1 & 0 & 0.756382 & -2.419811 & -0.004294 \\
\hline
\end{tabular}

\section{Model A: N-Methyl Enol}

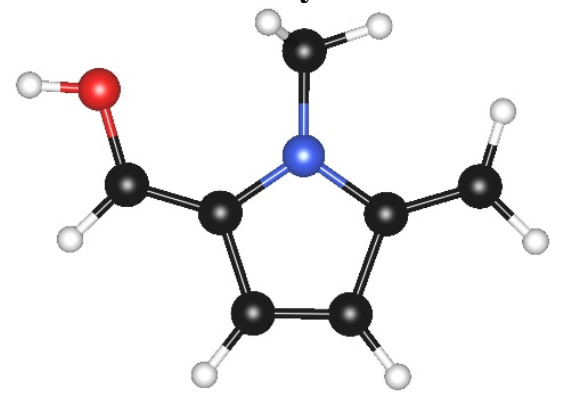

HF $(M 062 X / 6-31+G(d, p))=-401.9241129$ Hartrees

Imaginary Frequencies: none found

Zero-point correction $=0.147610$ (Hartree/Particle)

Thermal correction $=0.114830$ Hartrees

\section{Coordinates from last standard orientation:}

\begin{tabular}{|c|c|c|c|c|c|}
\hline \multirow{2}{*}{$\begin{array}{l}\text { Center } \\
\text { Number }\end{array}$} & \multirow{2}{*}{$\begin{array}{l}\text { Atomic } \\
\text { Number }\end{array}$} & \multirow{2}{*}{$\begin{array}{c}\text { Atomic } \\
\text { Type }\end{array}$} & \multicolumn{3}{|c|}{ Coordinates (Angstroms) } \\
\hline & & & $\mathrm{x}$ & $\mathrm{Y}$ & Z \\
\hline 1 & 6 & 0 & 1.558034 & -0.074061 & -0.000042 \\
\hline 2 & 6 & 0 & 1.513494 & 1.393769 & -0.000060 \\
\hline 3 & 1 & 0 & 2.401662 & 2.011126 & -0.000108 \\
\hline 4 & 6 & 0 & 0.231780 & 1.797207 & -0.000024 \\
\hline 5 & 1 & 0 & -0.143980 & 2.812141 & -0.000039 \\
\hline 6 & 6 & 0 & -0.628134 & 0.616561 & 0.000062 \\
\hline 7 & 6 & 0 & -1.973424 & 0.649482 & 0.000037 \\
\hline 8 & 1 & 0 & -2.485604 & 1.605266 & -0.000013 \\
\hline 9 & 6 & 0 & 2.670066 & -0.837289 & -0.000070 \\
\hline 10 & 1 & 0 & 2.652371 & -1.919093 & -0.000048 \\
\hline 11 & 1 & 0 & 3.636566 & -0.350580 & -0.000131 \\
\hline 12 & 8 & 0 & -2.744797 & -0.485499 & -0.000246 \\
\hline 13 & 1 & 0 & -3.675324 & -0.244956 & 0.001157 \\
\hline
\end{tabular}




\begin{tabular}{|c|c|c|c|c|c|}
\hline 14 & 7 & 0 & 0.225308 & -0.488008 & 0.000052 \\
\hline 15 & 6 & 0 & -0.212921 & -1.866870 & 0.000141 \\
\hline 16 & 1 & 0 & -0.811436 & -2.087405 & 0.888161 \\
\hline 17 & 1 & 0 & -0.811305 & -2.087584 & -0.887924 \\
\hline 18 & 1 & 0 & 0.664912 & -2.511655 & 0.000278 \\
\hline
\end{tabular}

\section{Model A: N-Methyl Iminium}

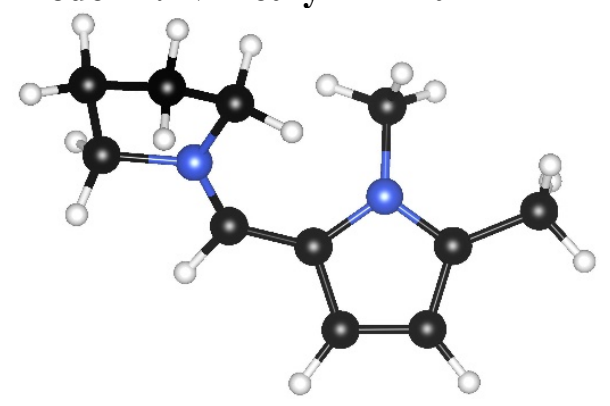

HF $(M 062 X / 6-31+G(d, p))=-538.4674297$ Hartrees

Imaginary Frequencies: none found

Zero-point correction $=0.270132$ (Hartree/Particle)

Thermal correction $=0.231458$ Hartrees

Coordinates from last standard orientation:

\begin{tabular}{|c|c|c|c|c|c|}
\hline \multirow{2}{*}{$\begin{array}{l}\text { Center } \\
\text { Number }\end{array}$} & \multirow{2}{*}{$\begin{array}{l}\text { Atomic } \\
\text { Number }\end{array}$} & \multirow{2}{*}{$\begin{array}{c}\text { Atomic } \\
\text { Type }\end{array}$} & \multicolumn{3}{|c|}{ Coordinates (Angstroms) } \\
\hline & & & $\mathrm{x}$ & $\mathrm{Y}$ & $\mathrm{Z}$ \\
\hline & & & 1 & (15 & ---------- \\
\hline 1 & 6 & 0 & -1.722837 & 1.857073 & 0.232348 \\
\hline 2 & 6 & 0 & -2.931929 & 1.229409 & 0.493860 \\
\hline 3 & 6 & 0 & -0.805995 & 0.868289 & -0.176819 \\
\hline 4 & 6 & 0 & 0.560658 & 1.139168 & -0.361636 \\
\hline 5 & 6 & 0 & -2.773592 & -0.128070 & 0.186240 \\
\hline 6 & 6 & 0 & -3.798586 & -1.209555 & 0.187535 \\
\hline 7 & 7 & 0 & 1.615022 & 0.367417 & -0.267930 \\
\hline 8 & 6 & 0 & 1.685857 & -0.960140 & 0.375870 \\
\hline 9 & 6 & 0 & 2.982235 & 0.907853 & -0.513902 \\
\hline 10 & 6 & 0 & 3.065431 & -0.933008 & 1.030289 \\
\hline 11 & 1 & 0 & 0.849155 & -1.065871 & 1.071087 \\
\hline 12 & 6 & 0 & 3.911550 & -0.202457 & -0.017598 \\
\hline 13 & 1 & 0 & 3.101931 & 1.136695 & -1.574624 \\
\hline 14 & 1 & 0 & 3.026351 & -0.362807 & 1.963721 \\
\hline 15 & 1 & 0 & 4.840653 & 0.200515 & 0.386843 \\
\hline 16 & 1 & 0 & 1.638069 & -1.748339 & -0.382025 \\
\hline 17 & 1 & 0 & 3.430103 & -1.936875 & 1.252589 \\
\hline 18 & 1 & 0 & 4.165178 & -0.879589 & -0.838607 \\
\hline 19 & 1 & 0 & 0.784982 & 2.183909 & -0.578904 \\
\hline 20 & 1 & 0 & -1.483234 & 2.903547 & 0.368125 \\
\hline 21 & 1 & 0 & -3.845453 & 1.681422 & 0.851624 \\
\hline 22 & 1 & 0 & -3.434089 & -2.111412 & 0.687288 \\
\hline 23 & 1 & 0 & 3.094202 & 1.828920 & 0.067222 \\
\hline 24 & 1 & 0 & -4.692529 & -0.863625 & 0.706803 \\
\hline 25 & 1 & 0 & -4.089087 & -1.479715 & -0.834051 \\
\hline 26 & 7 & 0 & -1.493089 & -0.347597 & -0.201778 \\
\hline
\end{tabular}




$\begin{array}{llllll}27 & 6 & 0 & -1.064931 & -1.569150 & -0.879793 \\ 28 & 1 & 0 & -0.223923 & -1.337833 & -1.532687 \\ 29 & 1 & 0 & -0.781418 & -2.344591 & -0.164033 \\ 30 & 1 & 0 & -1.881588 & -1.939556 & -1.500783 \\ - & 1 & 0 & -------1038\end{array}$

\section{Model A: N-Methyl Trienamine}

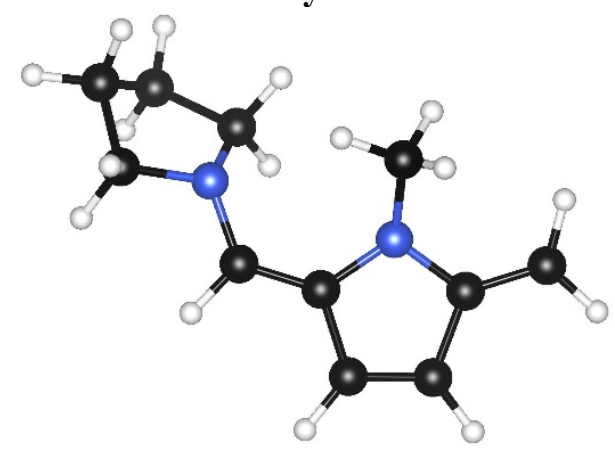

HF $(M 062 X / 6-31+G(d, p))=-538.036348$ Hartrees

Imaginary Frequencies: none found

Zero-point correction $=0.255373$ (Hartree/Particle)

Thermal correction $=0.216550$ Hartrees

Coordinates from last standard orientation:

\begin{tabular}{|c|c|c|c|c|c|}
\hline \multirow{2}{*}{$\begin{array}{l}\text { Center } \\
\text { Number }\end{array}$} & \multirow{2}{*}{$\begin{array}{l}\text { Atomic } \\
\text { Number }\end{array}$} & \multirow{2}{*}{$\begin{array}{c}\text { Atomic } \\
\text { Type }\end{array}$} & \multicolumn{3}{|c|}{ Coordinates (Angstroms) } \\
\hline & & & $\mathrm{x}$ & Y & Z \\
\hline 1 & 6 & 0 & 2.814713 & -0.290995 & 0.174884 \\
\hline 2 & 6 & 0 & 3.041271 & 1.135354 & 0.424505 \\
\hline 3 & 6 & 0 & 1.890383 & 1.802036 & 0.213702 \\
\hline 4 & 6 & 0 & 0.844891 & 0.846479 & -0.130048 \\
\hline 5 & 7 & 0 & 1.452245 & -0.422839 & -0.119433 \\
\hline 6 & 1 & 0 & 3.998097 & 1.543542 & 0.721410 \\
\hline 7 & 1 & 0 & 1.712587 & 2.864467 & 0.323323 \\
\hline 8 & 6 & 0 & -0.466044 & 1.151306 & -0.287805 \\
\hline 9 & 1 & 0 & -0.729474 & 2.207776 & -0.343159 \\
\hline 10 & 7 & 0 & -1.520895 & 0.247424 & -0.353478 \\
\hline 11 & 6 & 0 & 3.726602 & -1.285042 & 0.158615 \\
\hline 12 & 1 & 0 & 4.762619 & -1.063211 & 0.379788 \\
\hline 13 & 1 & 0 & 3.459920 & -2.311244 & -0.062630 \\
\hline 14 & 6 & 0 & -2.829785 & 0.783659 & -0.728474 \\
\hline 15 & 1 & 0 & -2.902276 & 0.912720 & -1.813030 \\
\hline 16 & 1 & 0 & -2.995164 & 1.768383 & -0.257387 \\
\hline 17 & 6 & 0 & -3.818778 & -0.241848 & -0.177894 \\
\hline 18 & 1 & 0 & -3.900208 & -1.091781 & -0.864468 \\
\hline 19 & 1 & 0 & -4.816455 & 0.177492 & -0.029747 \\
\hline 20 & 6 & 0 & -3.137271 & -0.676126 & 1.123125 \\
\hline 21 & 1 & 0 & -3.519594 & -1.618286 & 1.523197 \\
\hline 22 & 1 & 0 & -3.270119 & 0.099459 & 1.885641 \\
\hline 23 & 6 & 0 & -1.662979 & -0.762671 & 0.716618 \\
\hline 24 & 1 & 0 & -0.972097 & -0.540559 & 1.536400 \\
\hline 25 & 1 & 0 & -1.424492 & -1.764457 & 0.336867 \\
\hline 26 & 6 & 0 & 0.999916 & -1.540788 & -0.923578 \\
\hline 27 & 1 & 0 & 0.959721 & -2.459369 & -0.327726 \\
\hline
\end{tabular}




\begin{tabular}{|c|c|c|c|c|c|}
\hline 28 & 1 & 0 & 0.008023 & -1.323316 & -1.319312 \\
\hline 29 & 1 & 0 & 1.691956 & -1.701891 & -1.760688 \\
\hline
\end{tabular}

\section{Model B: N-Methyl Aldehyde}

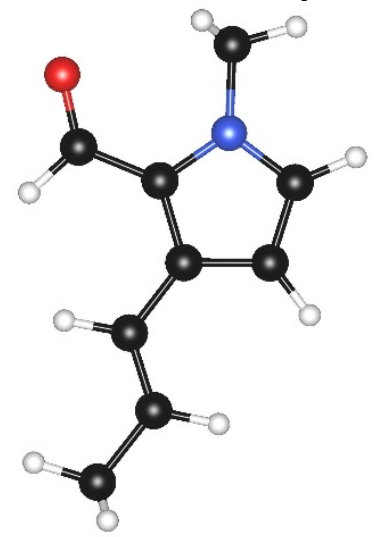

HF $(M 062 X / 6-31+G(d, p))=-479.3428132$ Hartrees

Imaginary Frequencies: none found Zero-point correction $=0.183139$ (Hartree/Particle) Thermal correction $=0.145447$ Hartrees

Coordinates from last standard orientation:

\begin{tabular}{|c|c|c|c|c|c|}
\hline \multirow{2}{*}{$\begin{array}{l}\text { Center } \\
\text { Number }\end{array}$} & \multirow{2}{*}{$\begin{array}{l}\text { Atomic } \\
\text { Number }\end{array}$} & \multirow{2}{*}{$\begin{array}{c}\text { Atomic } \\
\text { Type }\end{array}$} & \multicolumn{3}{|c|}{ Coordinates (Angstroms) } \\
\hline & & & $\mathrm{x}$ & $\mathrm{Y}$ & Z \\
\hline 1 & 6 & 0 & 0.500004 & -0.314085 & -0.000219 \\
\hline 2 & 6 & 0 & -0.757848 & 0.307683 & -0.000068 \\
\hline 3 & 6 & 0 & -1.070859 & 1.721983 & 0.000088 \\
\hline 4 & 1 & 0 & -0.190110 & 2.390109 & -0.000160 \\
\hline 5 & 6 & 0 & 1.794721 & 0.367839 & -0.000532 \\
\hline 6 & 6 & 0 & 2.983147 & -0.247639 & 0.000319 \\
\hline 7 & 6 & 0 & 4.303291 & 0.460140 & 0.000064 \\
\hline 8 & 1 & 0 & 4.895763 & 0.185613 & -0.879529 \\
\hline 9 & 1 & 0 & 4.170264 & 1.545221 & -0.001213 \\
\hline 10 & 1 & 0 & 1.781120 & 1.456549 & -0.001458 \\
\hline 11 & 1 & 0 & 3.015861 & -1.337018 & 0.001339 \\
\hline 12 & 6 & 0 & -1.112035 & -1.885584 & 0.000171 \\
\hline 13 & 1 & 0 & -1.696717 & -2.795127 & 0.000247 \\
\hline 14 & 6 & 0 & 0.258648 & -1.708013 & 0.000371 \\
\hline 15 & 1 & 0 & 0.992808 & -2.500095 & 0.000576 \\
\hline 16 & 1 & 0 & 4.895047 & 0.187585 & 0.880756 \\
\hline 17 & 8 & 0 & -2.196907 & 2.193140 & 0.000602 \\
\hline 18 & 7 & 0 & -1.725914 & -0.677391 & -0.000303 \\
\hline 19 & 6 & 0 & -3.168718 & -0.476452 & -0.000478 \\
\hline 20 & 1 & 0 & -3.475004 & 0.082162 & 0.884505 \\
\hline 21 & 1 & 0 & -3.474061 & 0.086527 & -0.882945 \\
\hline 22 & 1 & 0 & -3.640432 & -1.460144 & -0.003115 \\
\hline
\end{tabular}




\section{Model B: N-Methyl Enol}

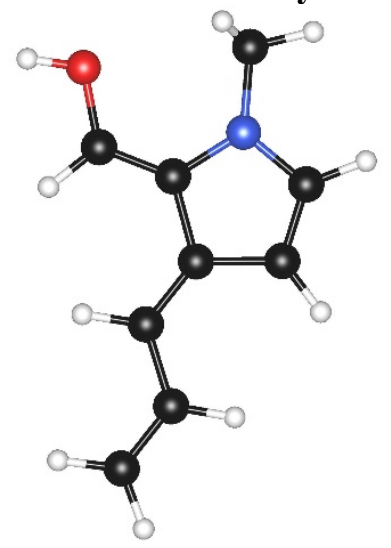

HF $(M 062 X / 6-31+G(d, p))=-479.2896605$ Hartrees

Imaginary Frequencies: none found

Zero-point correction $=0.181189$ (Hartree/Particle)

Thermal correction $=0.144177$ Hartrees

Coordinates from last standard orientation:

\begin{tabular}{|c|c|c|c|c|c|}
\hline \multirow{2}{*}{$\begin{array}{l}\text { Center } \\
\text { Number }\end{array}$} & \multirow{2}{*}{$\begin{array}{l}\text { Atomic } \\
\text { Number }\end{array}$} & \multirow{2}{*}{$\begin{array}{c}\text { Atomic } \\
\text { Type }\end{array}$} & \multicolumn{3}{|c|}{ Coordinates (Angstroms) } \\
\hline & & & $\mathrm{x}$ & Y & Z \\
\hline & & & & & \\
\hline 1 & 6 & 0 & 0.665774 & 0.250794 & -0.000134 \\
\hline 2 & 6 & 0 & -0.712518 & -0.310003 & -0.000597 \\
\hline 3 & 6 & 0 & -1.049688 & -1.610470 & 0.000066 \\
\hline 4 & 1 & 0 & -0.280281 & -2.374202 & 0.001512 \\
\hline 5 & 6 & 0 & 1.824424 & -0.459271 & -0.000076 \\
\hline 6 & 6 & 0 & 3.144230 & 0.132753 & 0.000294 \\
\hline 7 & 6 & 0 & 4.290461 & -0.568111 & 0.000396 \\
\hline 8 & 1 & 0 & 5.256317 & -0.075753 & 0.000672 \\
\hline 9 & 1 & 0 & 4.285298 & -1.655134 & 0.000198 \\
\hline 10 & 1 & 0 & 1.784728 & -1.547289 & -0.000295 \\
\hline 11 & 1 & 0 & 3.198655 & 1.220269 & 0.000499 \\
\hline 12 & 6 & 0 & -0.852054 & 1.940021 & -0.000964 \\
\hline 13 & 1 & 0 & -1.366095 & 2.893544 & -0.001157 \\
\hline 14 & 6 & 0 & 0.480328 & 1.696572 & 0.000109 \\
\hline 15 & 1 & 0 & 1.256616 & 2.445374 & 0.000512 \\
\hline 16 & 8 & 0 & -2.353921 & -2.034553 & 0.000206 \\
\hline 17 & 1 & 0 & -2.382784 & -2.995300 & -0.005005 \\
\hline 18 & 7 & 0 & -1.595456 & 0.782534 & -0.001649 \\
\hline 19 & 6 & 0 & -3.041348 & 0.718901 & 0.001760 \\
\hline 20 & 1 & 0 & -3.415399 & 0.197518 & -0.883308 \\
\hline 21 & 1 & 0 & -3.411580 & 0.200973 & 0.890545 \\
\hline 22 & 1 & 0 & -3.423565 & 1.741557 & 0.000595 \\
\hline
\end{tabular}




\section{Model B: N-Methyl Iminium}

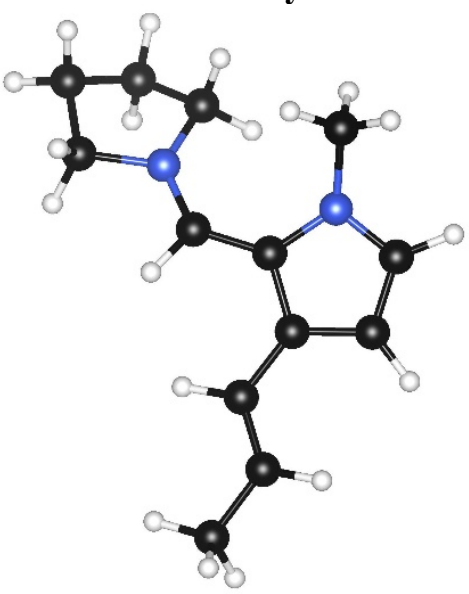

HF $(M 062 X / 6-31+G(d, p))=-615.8341523$ Hartrees

Imaginary Frequencies: none found

Zero-point correction $=0.303290$ (Hartree/Particle)

Thermal correction $=0.260398$ Hartrees

Coordinates from last standard orientation:

\begin{tabular}{|c|c|c|c|c|c|}
\hline \multirow{2}{*}{$\begin{array}{l}\text { Center } \\
\text { Number }\end{array}$} & \multirow{2}{*}{$\begin{array}{l}\text { Atomic } \\
\text { Number }\end{array}$} & \multirow{2}{*}{$\begin{array}{c}\text { Atomic } \\
\text { Type }\end{array}$} & \multicolumn{3}{|c|}{ Coordinates (Angstroms) } \\
\hline & & & $\mathrm{X}$ & Y & Z \\
\hline 1 & 6 & 0 & 1.811152 & 0.364035 & -0.055058 \\
\hline 2 & 6 & 0 & 0.424778 & 0.574691 & 0.192680 \\
\hline 3 & 6 & 0 & -0.475122 & -0.468409 & 0.463737 \\
\hline 4 & 1 & 0 & -0.015667 & -1.378795 & 0.848089 \\
\hline 5 & 7 & 0 & -1.773729 & -0.555436 & 0.304879 \\
\hline 6 & 6 & 0 & -2.507601 & -1.790214 & 0.700901 \\
\hline 7 & 1 & 0 & -2.514532 & -1.882130 & 1.788671 \\
\hline 8 & 1 & 0 & -1.983796 & -2.650426 & 0.271200 \\
\hline 9 & 6 & 0 & -3.899165 & -1.603635 & 0.092071 \\
\hline 10 & 1 & 0 & -4.567925 & -1.124564 & 0.813260 \\
\hline 11 & 1 & 0 & -4.342873 & -2.556578 & -0.198661 \\
\hline 12 & 6 & 0 & -3.645054 & -0.670443 & -1.096586 \\
\hline 13 & 1 & 0 & -4.546884 & -0.166905 & -1.447642 \\
\hline 14 & 1 & 0 & -3.204823 & -1.219390 & -1.934975 \\
\hline 15 & 6 & 0 & -2.627727 & 0.315913 & -0.525713 \\
\hline 16 & 1 & 0 & -2.006371 & 0.818940 & -1.271267 \\
\hline 17 & 1 & 0 & -3.128453 & 1.059805 & 0.101330 \\
\hline 18 & 6 & 0 & 2.493409 & -0.925472 & -0.071043 \\
\hline 19 & 6 & 0 & 3.827825 & -1.059563 & -0.078744 \\
\hline 20 & 6 & 0 & 4.547987 & -2.367305 & -0.090689 \\
\hline 21 & 1 & 0 & 5.201782 & -2.452928 & 0.783358 \\
\hline 22 & 1 & 0 & 3.854933 & -3.211368 & -0.094141 \\
\hline 23 & 1 & 0 & 1.886102 & -1.827931 & -0.079077 \\
\hline 24 & 1 & 0 & 4.449264 & -0.164388 & -0.070942 \\
\hline 25 & 6 & 0 & 1.344838 & 2.556787 & -0.201384 \\
\hline 26 & 1 & 0 & 1.390253 & 3.635623 & -0.274126 \\
\hline 27 & 6 & 0 & 2.361739 & 1.625499 & -0.330809 \\
\hline 28 & 1 & 0 & 3.382657 & 1.849249 & -0.601424 \\
\hline
\end{tabular}




$\begin{array}{rrrrrr}29 & 1 & 0 & 5.191459 & -2.439467 & -0.973749 \\ 30 & 7 & 0 & 0.176317 & 1.946768 & 0.091327 \\ 31 & 6 & 0 & -0.993990 & 2.675995 & 0.573483 \\ 32 & 1 & 0 & -1.699127 & 2.881071 & -0.233633 \\ 33 & 1 & 0 & -1.482117 & 2.101848 & 1.362638 \\ 34 & 1 & 0 & -0.660412 & 3.621739 & 1.000572 \\ -\end{array}$

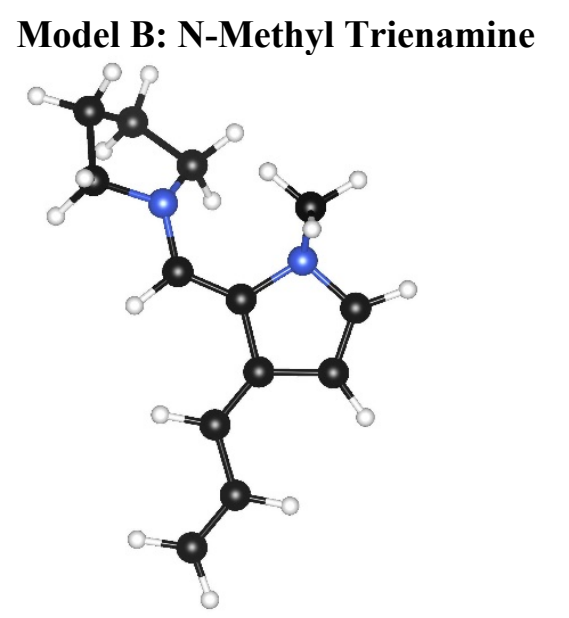

HF $(M 062 X / 6-31+G(d, p))=-615.4020067$ Hartrees

Imaginary Frequencies: none found

Zero-point correction $=0.289129$ (Hartree/Particle)

Thermal correction $=0.247231$ Hartrees

Coordinates from last standard orientation:

\begin{tabular}{|c|c|c|c|c|c|}
\hline \multirow{2}{*}{$\begin{array}{l}\text { Center } \\
\text { Number }\end{array}$} & \multirow{2}{*}{$\begin{array}{l}\text { Atomic } \\
\text { Number }\end{array}$} & \multirow{2}{*}{$\begin{array}{c}\text { Atomic } \\
\text { Type }\end{array}$} & \multicolumn{3}{|c|}{ Coordinates (Angstroms) } \\
\hline & & & $\mathrm{x}$ & $\mathrm{Y}$ & Z \\
\hline 1 & 6 & 0 & -1.897788 & 0.202946 & -0.007825 \\
\hline 2 & 6 & 0 & -0.442985 & 0.390763 & -0.174869 \\
\hline 3 & 6 & 0 & 0.479549 & -0.554031 & -0.471925 \\
\hline 4 & 1 & 0 & 0.135230 & -1.529418 & -0.816614 \\
\hline 5 & 7 & 0 & 1.847247 & -0.399223 & -0.341899 \\
\hline 6 & 6 & 0 & 2.712695 & -1.449128 & -0.877810 \\
\hline 7 & 1 & 0 & 2.773134 & -1.384303 & -1.968628 \\
\hline 8 & 1 & 0 & 2.321382 & -2.447179 & -0.615883 \\
\hline 9 & 6 & 0 & 4.051327 & -1.197903 & -0.187636 \\
\hline 10 & 1 & 0 & 4.593451 & -0.397974 & -0.703880 \\
\hline 11 & 1 & 0 & 4.686034 & -2.086560 & -0.168391 \\
\hline 12 & 6 & 0 & 3.614075 & -0.729778 & 1.204473 \\
\hline 13 & 1 & 0 & 4.398578 & -0.201578 & 1.751431 \\
\hline 14 & 1 & 0 & 3.303304 & -1.593672 & 1.802239 \\
\hline 15 & 6 & 0 & 2.403369 & 0.160634 & 0.905741 \\
\hline 16 & 1 & 0 & 1.643656 & 0.139563 & 1.693492 \\
\hline 17 & 1 & 0 & 2.706982 & 1.207031 & 0.767735 \\
\hline 18 & 6 & 0 & -2.579155 & -0.972044 & -0.050640 \\
\hline 19 & 6 & 0 & -4.008205 & -1.098884 & 0.137461 \\
\hline 20 & 6 & 0 & -4.681783 & -2.260097 & 0.081907 \\
\hline 21 & 1 & 0 & -5.755166 & -2.300651 & 0.228662 \\
\hline 22 & 1 & 0 & -4.169636 & -3.198498 & -0.114929 \\
\hline
\end{tabular}




$\begin{array}{rrrrrr}23 & 1 & 0 & -2.030163 & -1.894819 & -0.231439 \\ 24 & 1 & 0 & -4.565397 & -0.183962 & 0.333732 \\ 25 & 6 & 0 & -1.360755 & 2.388840 & 0.326830 \\ 26 & 1 & 0 & -1.365269 & 3.457920 & 0.504762 \\ 27 & 6 & 0 & -2.406964 & 1.539061 & 0.297216 \\ 28 & 1 & 0 & -3.433351 & 1.797873 & 0.512383 \\ 29 & 7 & 0 & -0.136679 & 1.757691 & 0.087299 \\ 30 & 6 & 0 & 0.755272 & 2.449718 & -0.842537 \\ 31 & 1 & 0 & 1.696030 & 1.906957 & -0.933790 \\ 32 & 1 & 0 & 0.302668 & 2.525193 & -1.841408 \\ 33 & 1 & 0 & 0.952645 & 3.454216 & -0.459594 \\ -------1.459\end{array}$

\section{Model C: N-Methyl Aldehyde}

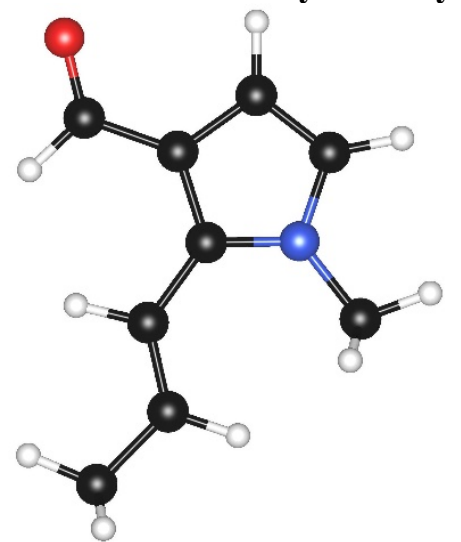

HF $(M 062 X / 6-31+G(d, p))=-479.3380203$ Hartrees

Imaginary Frequencies: none found

Zero-point correction $=0.183041$ (Hartree/Particle)

Thermal correction $=0.147182$ Hartrees

Coordinates from last standard orientation:

\begin{tabular}{|c|c|c|c|c|c|}
\hline \multirow{2}{*}{$\begin{array}{l}\text { Center } \\
\text { Number }\end{array}$} & \multirow{2}{*}{$\begin{array}{l}\text { Atomic } \\
\text { Number }\end{array}$} & \multirow{2}{*}{$\begin{array}{c}\text { Atomic } \\
\text { Type }\end{array}$} & \multicolumn{3}{|c|}{ Coordinates (Angstroms) } \\
\hline & & & $\mathrm{x}$ & $\mathrm{Y}$ & Z \\
\hline 1 & 6 & 0 & 0.004769 & 0.102861 & -0.000090 \\
\hline 2 & 6 & 0 & -1.333247 & -0.317855 & 0.000070 \\
\hline 3 & 6 & 0 & -1.825738 & -1.691553 & -0.000113 \\
\hline 4 & 1 & 0 & -1.063766 & -2.495822 & -0.000332 \\
\hline 5 & 6 & 0 & 1.182743 & -0.754720 & -0.000103 \\
\hline 6 & 6 & 0 & 2.492186 & -0.459614 & 0.000352 \\
\hline 7 & 6 & 0 & 3.563452 & -1.509072 & 0.000082 \\
\hline 8 & 1 & 0 & 4.209845 & -1.410275 & 0.879067 \\
\hline 9 & 1 & 0 & 3.134293 & -2.514402 & 0.002187 \\
\hline 10 & 1 & 0 & 0.930484 & -1.813388 & -0.000438 \\
\hline 11 & 1 & 0 & 2.842954 & 0.566860 & 0.000934 \\
\hline 12 & 6 & 0 & -1.317794 & 1.918362 & 0.000376 \\
\hline 13 & 1 & 0 & -1.524953 & 2.978692 & 0.000539 \\
\hline 14 & 6 & 0 & -2.158065 & 0.838715 & 0.000156 \\
\hline 15 & 1 & 0 & -3.237612 & 0.843382 & 0.000201 \\
\hline 16 & 1 & 0 & 4.206820 & -1.412755 & -0.881421 \\
\hline 17 & 8 & 0 & -3.005916 & -1.985007 & -0.000108 \\
\hline 18 & 7 & 0 & -0.012853 & 1.473727 & 0.000065 \\
\hline
\end{tabular}




$\begin{array}{rrrrrr}19 & 6 & 0 & 1.126807 & 2.370713 & -0.000467 \\ 20 & 1 & 0 & 1.737924 & 2.220838 & 0.893331 \\ 21 & 1 & 0 & 1.738232 & 2.219217 & -0.893762 \\ 22 & 1 & 0 & 0.752395 & 3.394593 & -0.001474 \\ - & 1 & --------1\end{array}$

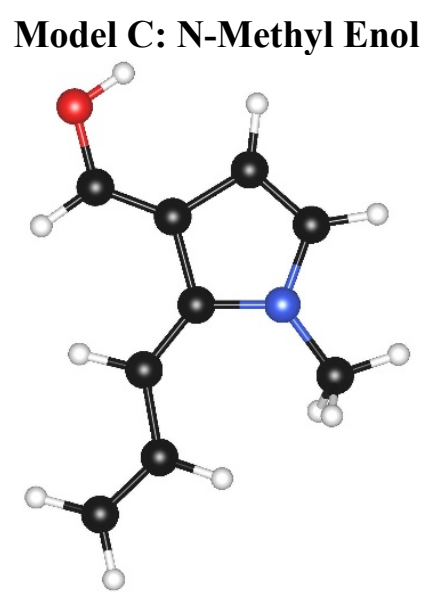

HF $(M 062 X / 6-31+G(d, p))=-479.2920517$ Hartrees

Imaginary Frequencies: none found

Zero-point correction $=0.182672$ (Hartree/Particle)

Thermal correction $=0.144988$ Hartrees

Coordinates from last standard orientation:

\begin{tabular}{|c|c|c|c|c|c|}
\hline \multirow{2}{*}{$\begin{array}{l}\text { Center } \\
\text { Number }\end{array}$} & \multirow{2}{*}{$\begin{array}{l}\text { Atomic } \\
\text { Number }\end{array}$} & \multirow{2}{*}{$\begin{array}{c}\text { Atomic } \\
\text { Type }\end{array}$} & \multicolumn{3}{|c|}{ Coordinates (Angstroms) } \\
\hline & & & $\mathrm{x}$ & Y & Z \\
\hline 1 & 6 & 0 & -0.159835 & 0.040163 & -0.012213 \\
\hline 2 & 6 & 0 & 1.298875 & -0.242662 & 0.016211 \\
\hline 3 & 6 & 0 & 1.866239 & -1.463406 & -0.028807 \\
\hline 4 & 1 & 0 & 1.296380 & -2.382950 & -0.093708 \\
\hline 5 & 6 & 0 & -1.158385 & -0.882390 & 0.046348 \\
\hline 6 & 6 & 0 & -2.595569 & -0.729682 & -0.064629 \\
\hline 7 & 6 & 0 & -3.455470 & -1.759700 & 0.020554 \\
\hline 8 & 1 & 0 & -4.524664 & -1.611725 & -0.078871 \\
\hline 9 & 1 & 0 & -3.105521 & -2.774628 & 0.190247 \\
\hline 10 & 1 & 0 & -0.825869 & -1.909834 & 0.175056 \\
\hline 11 & 1 & 0 & -3.010982 & 0.256766 & -0.243185 \\
\hline 12 & 6 & 0 & 1.011372 & 1.995784 & -0.024243 \\
\hline 13 & 1 & 0 & 1.109746 & 3.073488 & -0.042324 \\
\hline 14 & 6 & 0 & 1.974159 & 1.055352 & 0.036021 \\
\hline 15 & 1 & 0 & 3.036306 & 1.252273 & 0.059505 \\
\hline 16 & 8 & 0 & 3.198428 & -1.706650 & -0.007444 \\
\hline 17 & 1 & 0 & 3.685999 & -0.877570 & 0.071280 \\
\hline 18 & 7 & 0 & -0.256947 & 1.433880 & -0.083770 \\
\hline 19 & 6 & 0 & -1.467712 & 2.207432 & 0.061001 \\
\hline 20 & 1 & 0 & -2.113882 & 2.110239 & -0.816817 \\
\hline 21 & 1 & 0 & -2.023579 & 1.896136 & 0.952562 \\
\hline 22 & 1 & 0 & -1.194777 & 3.258501 & 0.170740 \\
\hline
\end{tabular}




\section{Model C: N-Methyl Iminium}

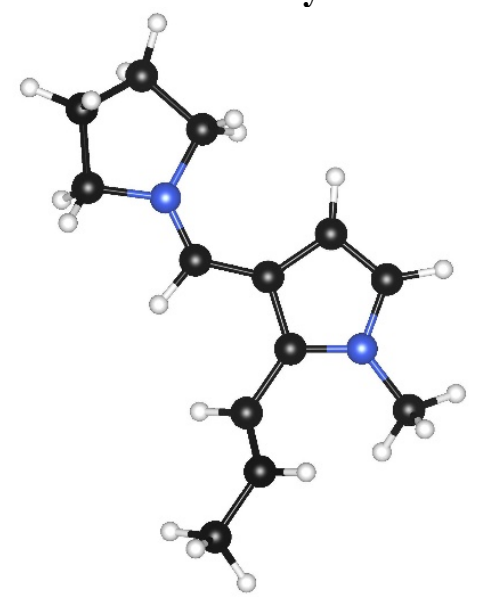

HF $(M 062 X / 6-31+G(d, p))=-615.8505083$ Hartrees

Imaginary Frequencies: none found

Zero-point correction $=0.303749$ (Hartree/Particle)

Thermal correction $=0.261707$ Hartrees

Coordinates from last standard orientation:

\begin{tabular}{|c|c|c|c|c|c|}
\hline \multirow{2}{*}{$\begin{array}{l}\text { Center } \\
\text { Number }\end{array}$} & \multirow{2}{*}{$\begin{array}{l}\text { Atomic } \\
\text { Number }\end{array}$} & \multirow{2}{*}{$\begin{array}{l}\text { Atomic } \\
\text { Type }\end{array}$} & \multicolumn{3}{|c|}{ Coordinates (Angstroms) } \\
\hline & & & $\mathrm{X}$ & Y & z \\
\hline & & & - & --- & ---------- \\
\hline 1 & 6 & 0 & -1.492579 & -0.248818 & -0.134969 \\
\hline 2 & 6 & 0 & -0.106471 & -0.545752 & -0.044852 \\
\hline 3 & 6 & 0 & 0.845996 & 0.492826 & -0.079440 \\
\hline 4 & 1 & 0 & 0.463237 & 1.512099 & -0.122783 \\
\hline 5 & 7 & 0 & 2.146619 & 0.402288 & -0.055137 \\
\hline 6 & 6 & 0 & 3.040332 & 1.587914 & -0.057134 \\
\hline 7 & 1 & 0 & 2.637574 & 2.353537 & 0.608060 \\
\hline 8 & 1 & 0 & 3.094140 & 1.982647 & -1.077527 \\
\hline 9 & 6 & 0 & 4.380628 & 1.009326 & 0.386824 \\
\hline 10 & 1 & 0 & 4.418118 & 0.939738 & 1.478511 \\
\hline 11 & 1 & 0 & 5.219397 & 1.621265 & 0.052900 \\
\hline 12 & 6 & 0 & 4.366642 & -0.388276 & -0.241140 \\
\hline 13 & 1 & 0 & 5.083468 & -1.072644 & 0.213990 \\
\hline 14 & 1 & 0 & 4.586690 & -0.325364 & -1.311253 \\
\hline 15 & 6 & 0 & 2.924294 & -0.853118 & -0.030418 \\
\hline 16 & 1 & 0 & 2.556917 & -1.523554 & -0.811169 \\
\hline 17 & 1 & 0 & 2.786596 & -1.327467 & 0.947935 \\
\hline 18 & 6 & 0 & -2.129328 & 1.051907 & -0.349932 \\
\hline 19 & 6 & 0 & -3.221478 & 1.480179 & 0.298362 \\
\hline 20 & 6 & 0 & -3.870678 & 2.807166 & 0.073872 \\
\hline 21 & 1 & 0 & -3.890354 & 3.385604 & 1.002847 \\
\hline 22 & 1 & 0 & -3.349563 & 3.385767 & -0.691773 \\
\hline 23 & 1 & 0 & -1.659682 & 1.706290 & -1.082344 \\
\hline 24 & 1 & 0 & -3.673048 & 0.839101 & 1.055645 \\
\hline 25 & 6 & 0 & -1.260697 & -2.456948 & 0.177818 \\
\hline 26 & 1 & 0 & -1.621559 & -3.467156 & 0.303241 \\
\hline 27 & 6 & 0 & 0.007616 & -1.966850 & 0.156851 \\
\hline 28 & 1 & 0 & 0.899269 & -2.557326 & 0.290046 \\
\hline 29 & 1 & 0 & -4.912018 & 2.675508 & -0.237729 \\
\hline 30 & 7 & 0 & -2.158173 & -1.415890 & -0.007720 \\
\hline
\end{tabular}




$\begin{array}{rlllrr}31 & 6 & 0 & -3.599271 & -1.629951 & -0.112625 \\ 32 & 1 & 0 & -4.072807 & -1.560828 & 0.869113 \\ 33 & 1 & 0 & -4.028311 & -0.884851 & -0.782080 \\ 34 & 1 & 0 & -3.767221 & -2.624777 & -0.524930\end{array}$

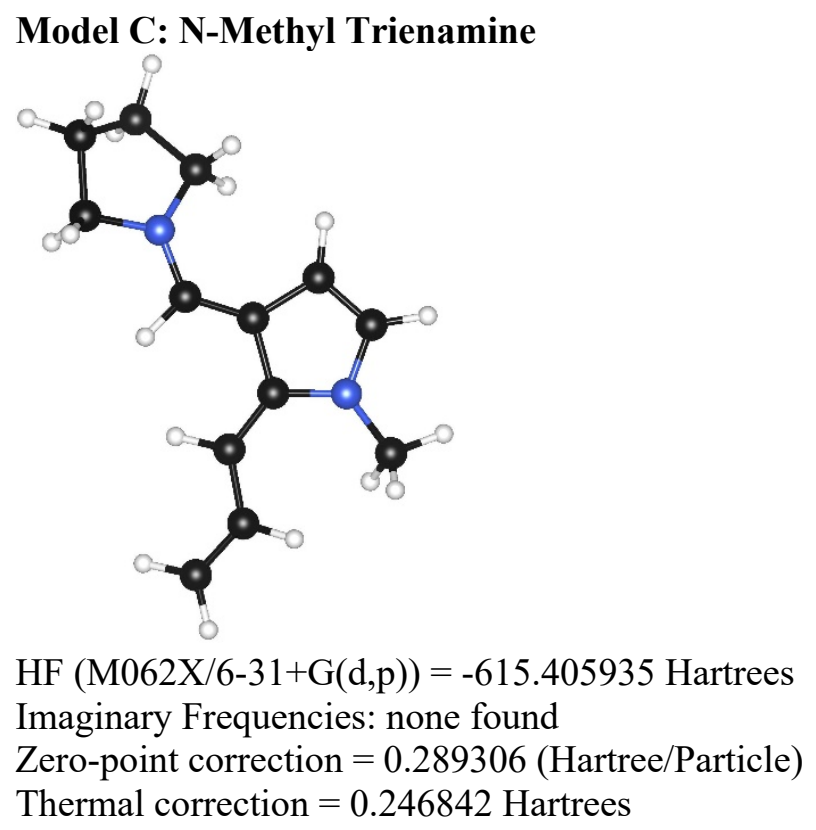

Coordinates from last standard orientation:

\begin{tabular}{|c|c|c|c|c|c|}
\hline \multirow{2}{*}{$\begin{array}{l}\text { Center } \\
\text { Number }\end{array}$} & \multirow{2}{*}{$\begin{array}{l}\text { Atomic } \\
\text { Number }\end{array}$} & \multirow{2}{*}{$\begin{array}{c}\text { Atomic } \\
\text { Type }\end{array}$} & \multicolumn{3}{|c|}{ Coordinates (Angstroms) } \\
\hline & & & $x$ & Y & Z \\
\hline 1 & 6 & 0 & 1.567162 & -0.122782 & -0.027991 \\
\hline 2 & 6 & 0 & 0.127385 & -0.449763 & -0.067520 \\
\hline 3 & 6 & 0 & -0.837610 & 0.504419 & -0.211371 \\
\hline 4 & 1 & 0 & -0.519833 & 1.533954 & -0.370908 \\
\hline 5 & 7 & 0 & -2.184581 & 0.342239 & -0.198725 \\
\hline 6 & 6 & 0 & -3.089846 & 1.467203 & -0.431620 \\
\hline 7 & 1 & 0 & -2.916051 & 1.910900 & -1.416991 \\
\hline 8 & 1 & 0 & -2.937920 & 2.247837 & 0.329405 \\
\hline 9 & 6 & 0 & -4.476270 & 0.835159 & -0.303145 \\
\hline 10 & 1 & 0 & -4.774784 & 0.393911 & -1.260168 \\
\hline 11 & 1 & 0 & -5.238564 & 1.558725 & -0.006503 \\
\hline 12 & 6 & 0 & -4.248853 & -0.269003 & 0.735949 \\
\hline 13 & 1 & 0 & -5.025903 & -1.036543 & 0.728860 \\
\hline 14 & 1 & 0 & -4.205196 & 0.166688 & 1.740033 \\
\hline 15 & 6 & 0 & -2.873235 & -0.820769 & 0.351090 \\
\hline 16 & 1 & 0 & -2.311377 & -1.216504 & 1.204505 \\
\hline 17 & 1 & 0 & -2.964215 & -1.618272 & -0.400048 \\
\hline 18 & 6 & 0 & 2.120132 & 1.112444 & 0.177903 \\
\hline 19 & 6 & 0 & 3.485308 & 1.547447 & -0.016181 \\
\hline 20 & 6 & 0 & 3.927816 & 2.795077 & 0.231856 \\
\hline 21 & 1 & 0 & 4.956911 & 3.077054 & 0.041041 \\
\hline 22 & 1 & 0 & 3.264640 & 3.560574 & 0.626503 \\
\hline 23 & 1 & 0 & 1.425612 & 1.890951 & 0.483903 \\
\hline
\end{tabular}




$\begin{array}{rrrrrr}24 & 1 & 0 & 4.199350 & 0.837924 & -0.425631 \\ 25 & 6 & 0 & 1.297422 & -2.376852 & -0.205840 \\ 26 & 1 & 0 & 1.645478 & -3.397238 & -0.296649 \\ 27 & 6 & 0 & 0.036558 & -1.907051 & -0.123599 \\ 28 & 1 & 0 & -0.857354 & -2.508527 & -0.174329 \\ 29 & 7 & 0 & 2.230401 & -1.340062 & -0.191077 \\ 30 & 6 & 0 & 3.626202 & -1.566946 & 0.112081 \\ 31 & 1 & 0 & 4.277572 & -1.286390 & -0.721068 \\ 32 & 1 & 0 & 3.916588 & -0.992380 & 0.999055 \\ 33 & 1 & 0 & 3.771273 & -2.629401 & 0.317926\end{array}$

\section{Model D: N-Methyl Aldehyde}

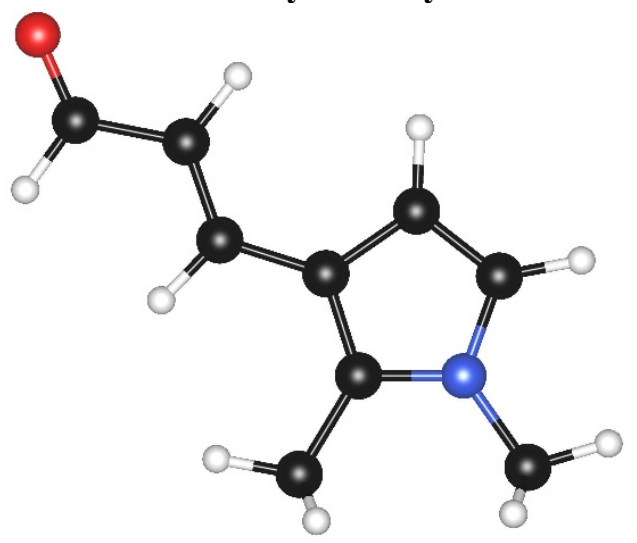

HF $(M 062 X / 6-31+G(d, p))=-479.3435913$ Hartrees

Imaginary Frequencies: none found

Zero-point correction $=0.182803$ (Hartree/Particle)

Thermal correction $=0.145068$ Hartrees

\section{Coordinates from last standard orientation:}

\begin{tabular}{|c|c|c|c|c|c|}
\hline \multirow{2}{*}{$\begin{array}{l}\text { Center } \\
\text { Number }\end{array}$} & \multirow{2}{*}{$\begin{array}{l}\text { Atomic } \\
\text { Number }\end{array}$} & \multirow{2}{*}{$\begin{array}{c}\text { Atomic } \\
\text { Type }\end{array}$} & \multicolumn{3}{|c|}{ Coordinates (Angstroms) } \\
\hline & & & $\mathrm{X}$ & Y & Z \\
\hline 1 & 6 & 0 & -1.237343 & 0.373788 & -0.000219 \\
\hline 2 & 6 & 0 & -2.391076 & -0.324071 & -0.000067 \\
\hline 3 & 6 & 0 & -3.671192 & 0.381889 & -0.000239 \\
\hline 4 & 1 & 0 & -3.592702 & 1.491658 & -0.000539 \\
\hline 5 & 1 & 0 & -2.422672 & -1.410171 & 0.000207 \\
\hline 6 & 1 & 0 & -1.318307 & 1.462318 & -0.000473 \\
\hline 7 & 6 & 0 & 0.100132 & -0.173031 & -0.000088 \\
\hline 8 & 6 & 0 & 1.277036 & 0.573464 & 0.000027 \\
\hline 9 & 6 & 0 & 0.481336 & -1.553053 & -0.000055 \\
\hline 10 & 6 & 0 & 1.848317 & -1.595298 & 0.000089 \\
\hline 11 & 1 & 0 & -0.179188 & -2.407638 & -0.000154 \\
\hline 12 & 1 & 0 & 2.532608 & -2.431051 & 0.000140 \\
\hline 13 & 6 & 0 & 1.506464 & 2.048874 & 0.000099 \\
\hline 14 & 1 & 0 & 2.067059 & 2.369716 & -0.885015 \\
\hline 15 & 1 & 0 & 0.555836 & 2.582972 & -0.000075 \\
\hline 16 & 1 & 0 & 2.066711 & 2.369679 & 0.885448 \\
\hline 17 & 8 & 0 & -4.760538 & -0.154877 & -0.000088 \\
\hline 18 & 7 & 0 & 2.325105 & -0.302266 & 0.000134 \\
\hline 19 & 6 & 0 & 3.724815 & 0.074242 & 0.000312 \\
\hline
\end{tabular}




$\begin{array}{rrrrrr}20 & 1 & 0 & 3.969537 & 0.659716 & -0.890337 \\ 21 & 1 & 0 & 3.969342 & 0.659608 & 0.891087 \\ 22 & 1 & 0 & 4.329408 & -0.832747 & 0.000322 \\ - & - & - & -\end{array}$

\section{Model D: N-Methyl Enol}

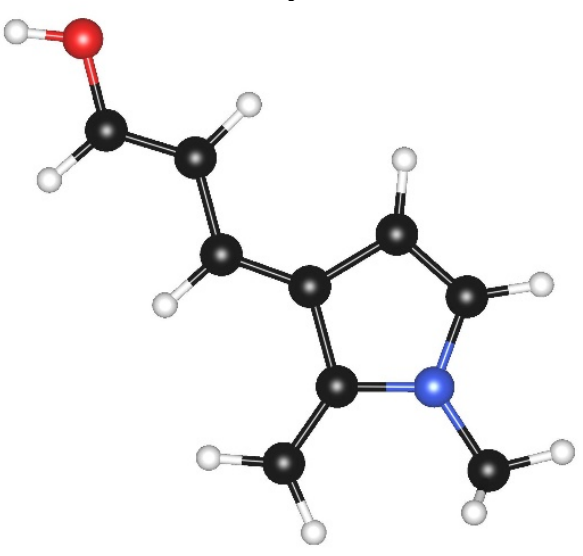

HF $(M 062 X / 6-31+G(d, p))=-479.2982428$ Hartrees

Imaginary Frequencies: none found

Zero-point correction $=-479.2982428$ (Hartree/Particle)

Thermal correction $=0.142483$ Hartrees

Coordinates from last standard orientation:

\begin{tabular}{|c|c|c|c|c|c|}
\hline \multirow{2}{*}{$\begin{array}{l}\text { Center } \\
\text { Number }\end{array}$} & \multirow{2}{*}{$\begin{array}{l}\text { Atomic } \\
\text { Number }\end{array}$} & \multirow{2}{*}{$\begin{array}{c}\text { Atomic } \\
\text { Type }\end{array}$} & \multicolumn{3}{|c|}{ Coordinates (Angstroms) } \\
\hline & & & $\mathrm{x}$ & Y & Z \\
\hline 1 & 6 & 0 & -1.090570 & 0.505750 & -0.000676 \\
\hline 2 & 6 & 0 & -2.338077 & -0.221732 & -0.001024 \\
\hline 3 & 6 & 0 & -3.526821 & 0.399357 & 0.000769 \\
\hline 4 & 1 & 0 & -3.608443 & 1.485261 & 0.002905 \\
\hline 5 & 1 & 0 & -2.327176 & -1.308579 & -0.002772 \\
\hline 6 & 1 & 0 & -1.156611 & 1.593184 & -0.000123 \\
\hline 7 & 6 & 0 & 0.138366 & -0.062725 & -0.000690 \\
\hline 8 & 6 & 0 & 1.428188 & 0.682719 & -0.000196 \\
\hline 9 & 6 & 0 & 0.498844 & -1.478508 & -0.000580 \\
\hline 10 & 6 & 0 & 1.847996 & -1.551346 & 0.000352 \\
\hline 11 & 1 & 0 & -0.178877 & -2.318081 & -0.000801 \\
\hline 12 & 1 & 0 & 2.484832 & -2.426992 & 0.000881 \\
\hline 13 & 6 & 0 & 1.660634 & 2.010181 & -0.000669 \\
\hline 14 & 1 & 0 & 2.665863 & 2.413280 & -0.000223 \\
\hline 15 & 1 & 0 & 0.837221 & 2.712323 & -0.001624 \\
\hline 16 & 8 & 0 & -4.695147 & -0.298886 & -0.000975 \\
\hline 17 & 1 & 0 & -5.446162 & 0.301249 & 0.008073 \\
\hline 18 & 7 & 0 & 2.425921 & -0.293256 & 0.000718 \\
\hline 19 & 6 & 0 & 3.831279 & 0.007275 & 0.001354 \\
\hline 20 & 1 & 0 & 4.107535 & 0.585968 & -0.888955 \\
\hline 21 & 1 & 0 & 4.106474 & 0.586872 & 0.891397 \\
\hline 22 & 1 & 0 & 4.396040 & -0.926431 & 0.002173 \\
\hline
\end{tabular}




\section{Model D: N-Methyl Iminium}

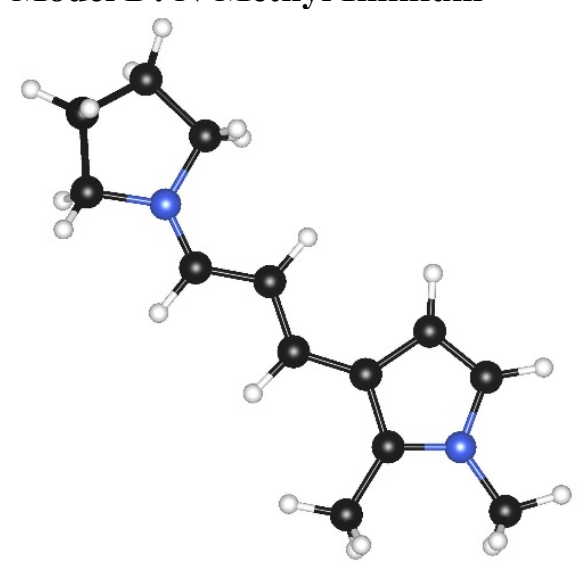

HF $(M 062 X / 6-31+G(d, p))=-615.8593694$ Hartrees

Imaginary Frequencies: none found

Zero-point correction $=0.303228$ (Hartree/Particle)

Thermal correction $=0.259681$ Hartrees

Coordinates from last standard orientation:

\begin{tabular}{|c|c|c|c|c|c|}
\hline \multirow{2}{*}{$\begin{array}{l}\text { Center } \\
\text { Number }\end{array}$} & \multirow{2}{*}{$\begin{array}{l}\text { Atomic } \\
\text { Number }\end{array}$} & \multirow{2}{*}{$\begin{array}{c}\text { Atomic } \\
\text { Type }\end{array}$} & \multicolumn{3}{|c|}{ Coordinates (Angstroms) } \\
\hline & & & $x$ & $Y$ & Z \\
\hline & & & & & \\
\hline 1 & 6 & 0 & 0.591563 & 0.557354 & 0.033993 \\
\hline 2 & 6 & 0 & -0.646211 & -0.050408 & 0.041316 \\
\hline 3 & 6 & 0 & -1.802418 & 0.748843 & 0.066576 \\
\hline 4 & 1 & 0 & -1.689921 & 1.833045 & 0.078085 \\
\hline 5 & 7 & 0 & -3.035160 & 0.311756 & 0.073286 \\
\hline 6 & 6 & 0 & -4.226609 & 1.188913 & 0.075746 \\
\hline 7 & 1 & 0 & -4.049491 & 2.056569 & -0.562665 \\
\hline 8 & 1 & 0 & -4.415620 & 1.526221 & 1.101156 \\
\hline 9 & 6 & 0 & -5.333019 & 0.258954 & -0.415332 \\
\hline 10 & 1 & 0 & -5.316765 & 0.200761 & -1.508239 \\
\hline 11 & 1 & 0 & -6.321356 & 0.598334 & -0.102947 \\
\hline 12 & 6 & 0 & -4.936635 & -1.087835 & 0.201028 \\
\hline 13 & 1 & 0 & -5.410420 & -1.939801 & -0.287938 \\
\hline 14 & 1 & 0 & -5.204877 & -1.111553 & 1.261609 \\
\hline 15 & 6 & 0 & -3.413888 & -1.116112 & 0.045852 \\
\hline 16 & 1 & 0 & -2.902441 & -1.650811 & 0.850440 \\
\hline 17 & 1 & 0 & -3.103464 & -1.539360 & -0.916483 \\
\hline 18 & 1 & 0 & -0.731896 & -1.131527 & 0.023416 \\
\hline 19 & 1 & 0 & 0.608179 & 1.647533 & 0.052860 \\
\hline 20 & 6 & 0 & 1.839014 & -0.096690 & 0.005331 \\
\hline 21 & 6 & 0 & 3.090973 & 0.559984 & 0.002311 \\
\hline 22 & 6 & 0 & 2.109311 & -1.510249 & -0.026016 \\
\hline 23 & 6 & 0 & 3.460394 & -1.653643 & -0.046127 \\
\hline 24 & 1 & 0 & 1.389006 & -2.314664 & -0.033371 \\
\hline 25 & 1 & 0 & 4.080458 & -2.537441 & -0.070707 \\
\hline 26 & 6 & 0 & 3.436662 & 2.009655 & 0.027837 \\
\hline 27 & 1 & 0 & 4.019158 & 2.290736 & -0.855454 \\
\hline 28 & 1 & 0 & 2.539233 & 2.627704 & 0.045328 \\
\hline 29 & 1 & 0 & 4.030660 & 2.257099 & 0.913572 \\
\hline 30 & 7 & 0 & 4.043507 & -0.390972 & -0.028507 \\
\hline 31 & 6 & 0 & 5.479133 & -0.141465 & -0.040817 \\
\hline
\end{tabular}




\begin{tabular}{|c|c|c|c|c|c|}
\hline 32 & 1 & 0 & 5.757060 & 0.434129 & -0.926473 \\
\hline 33 & 1 & 0 & 5.777568 & 0.402998 & 0.857831 \\
\hline 34 & 1 & 0 & 5.996883 & -1.099280 & -0.063655 \\
\hline
\end{tabular}

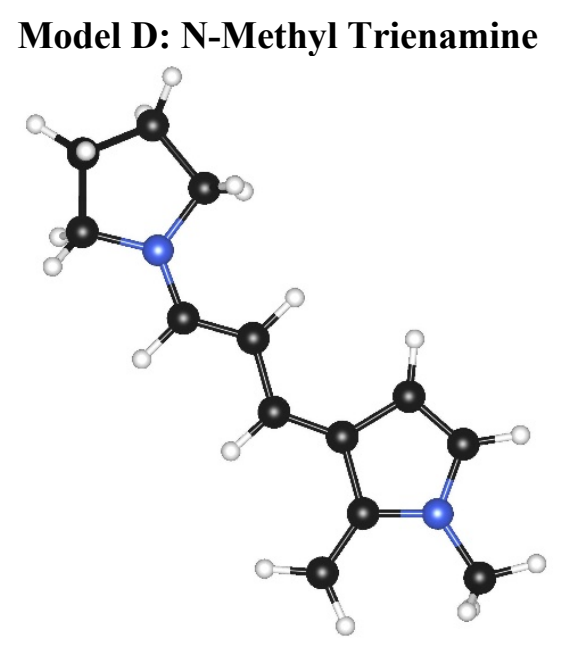

HF $(M 062 X / 6-31+G(d, p))=-615.4153755$ Hartrees

Imaginary Frequencies: none found

Zero-point correction $=0.287821$ (Hartree/Particle)

Thermal correction $=0.243096$ Hartrees

Coordinates from last standard orientation:

\begin{tabular}{|c|c|c|c|c|c|}
\hline \multirow{2}{*}{$\begin{array}{l}\text { Center } \\
\text { Number }\end{array}$} & \multirow{2}{*}{$\begin{array}{l}\text { Atomic } \\
\text { Number }\end{array}$} & \multirow{2}{*}{$\begin{array}{c}\text { Atomic } \\
\text { Type }\end{array}$} & \multicolumn{3}{|c|}{ Coordinates (Angstroms) } \\
\hline & & & $\mathrm{x}$ & $\mathrm{Y}$ & $\mathrm{z}$ \\
\hline 1 & 6 & 0 & 0.662053 & 0.669909 & 0.033299 \\
\hline 2 & 6 & 0 & -0.637146 & 0.055624 & 0.041815 \\
\hline 3 & 6 & 0 & -1.777819 & 0.798394 & 0.053959 \\
\hline 4 & 1 & 0 & -1.707435 & 1.885610 & 0.057001 \\
\hline 5 & 7 & 0 & -3.047713 & 0.319702 & 0.055991 \\
\hline 6 & 6 & 0 & -4.238308 & 1.159603 & 0.053140 \\
\hline 7 & 1 & 0 & -4.111028 & 2.007883 & -0.627545 \\
\hline 8 & 1 & 0 & -4.450930 & 1.553964 & 1.058735 \\
\hline 9 & 6 & 0 & -5.333427 & 0.191473 & -0.398118 \\
\hline 10 & 1 & 0 & -5.333920 & 0.119452 & -1.491002 \\
\hline 11 & 1 & 0 & -6.329130 & 0.499521 & -0.071704 \\
\hline 12 & 6 & 0 & -4.878924 & -1.138639 & 0.216492 \\
\hline 13 & 1 & 0 & -5.329544 & -2.011062 & -0.261259 \\
\hline 14 & 1 & 0 & -5.136511 & -1.163110 & 1.280736 \\
\hline 15 & 6 & 0 & -3.354805 & -1.101092 & 0.053059 \\
\hline 16 & 1 & 0 & -2.830564 & -1.612963 & 0.870314 \\
\hline 17 & 1 & 0 & -3.026350 & -1.558912 & -0.892852 \\
\hline 18 & 1 & 0 & -0.699494 & -1.029057 & 0.034619 \\
\hline 19 & 1 & 0 & 0.684827 & 1.759953 & 0.044206 \\
\hline 20 & 6 & 0 & 1.849610 & 0.012669 & 0.011699 \\
\hline 21 & 6 & 0 & 3.188139 & 0.657455 & 0.004070 \\
\hline 22 & 6 & 0 & 2.098773 & -1.426203 & -0.018474 \\
\hline 23 & 6 & 0 & 3.437427 & -1.605628 & -0.052625 \\
\hline 24 & 1 & 0 & 1.356327 & -2.209735 & -0.022414 \\
\hline
\end{tabular}




$\begin{array}{rrrrrr}25 & 1 & 0 & 4.007544 & -2.525530 & -0.084085 \\ 26 & 6 & 0 & 3.526752 & 1.963752 & 0.046855 \\ 27 & 1 & 0 & 4.560050 & 2.287923 & 0.035751 \\ 28 & 1 & 0 & 2.761448 & 2.727299 & 0.096084 \\ 29 & 7 & 0 & 4.107752 & -0.391723 & -0.050156 \\ 30 & 6 & 0 & 5.531024 & -0.199120 & -0.038203 \\ 31 & 1 & 0 & 5.849122 & 0.403242 & -0.898173 \\ 32 & 1 & 0 & 5.851048 & 0.311875 & 0.879266 \\ 33 & 1 & 0 & 6.024179 & -1.171382 & -0.090333 \\ -\end{array}$

\section{Model E: N-Methyl Aldehyde}

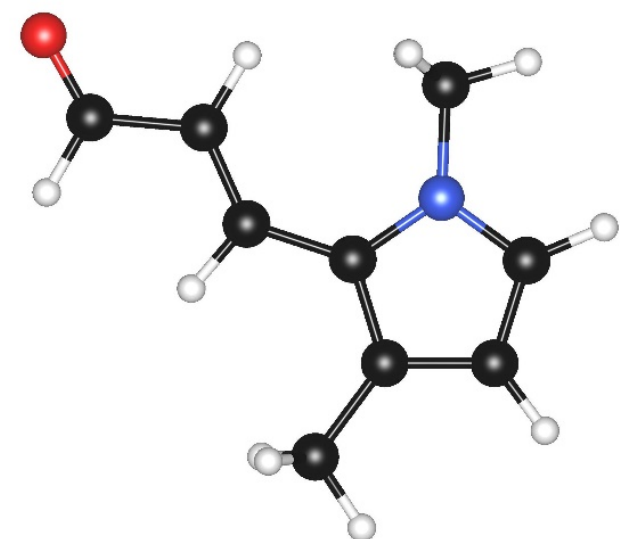

HF $(M 062 X / 6-31+G(d, p))=-479.3409082$ Hartrees

Imaginary Frequencies: none found

Zero-point correction $=0.183048$ (Hartree/Particle)

Thermal correction $=0.145284$ Hartrees

Coordinates from last standard orientation:

\begin{tabular}{|c|c|c|c|c|c|}
\hline \multirow{2}{*}{$\begin{array}{l}\text { Center } \\
\text { Number }\end{array}$} & \multirow{2}{*}{$\begin{array}{l}\text { Atomic } \\
\text { Number }\end{array}$} & \multirow{2}{*}{$\begin{array}{c}\text { Atomic } \\
\text { Type }\end{array}$} & \multicolumn{3}{|c|}{ Coordinates (Angstroms) } \\
\hline & & & $\mathrm{x}$ & Y & Z \\
\hline 1 & 6 & 0 & -0.791688 & -0.532842 & -0.007204 \\
\hline 2 & 6 & 0 & -1.951295 & 0.165128 & 0.004469 \\
\hline 3 & 6 & 0 & -3.219320 & -0.564208 & -0.003544 \\
\hline 4 & 1 & 0 & -3.124920 & -1.672095 & -0.020895 \\
\hline 5 & 1 & 0 & -2.026681 & 1.245417 & 0.021398 \\
\hline 6 & 1 & 0 & -0.895811 & -1.619722 & -0.020772 \\
\hline 7 & 6 & 0 & 0.584007 & -0.123074 & -0.003392 \\
\hline 8 & 6 & 0 & 1.689244 & -0.982161 & -0.001047 \\
\hline 9 & 6 & 0 & 2.422988 & 1.143198 & 0.004610 \\
\hline 10 & 1 & 0 & 2.992552 & 2.061840 & 0.005905 \\
\hline 11 & 6 & 0 & 1.647132 & -2.481870 & 0.001421 \\
\hline 12 & 1 & 0 & 1.188209 & -2.873517 & 0.915069 \\
\hline 13 & 1 & 0 & 1.083990 & -2.874545 & -0.851246 \\
\hline 14 & 6 & 0 & 2.843737 & -0.174957 & 0.004604 \\
\hline 15 & 1 & 0 & 3.869623 & -0.514985 & 0.007255 \\
\hline 16 & 1 & 0 & 2.660294 & -2.885261 & -0.058606 \\
\hline 17 & 8 & 0 & -4.314881 & -0.040466 & 0.007696 \\
\hline 18 & 7 & 0 & 1.065737 & 1.176498 & -0.000114 \\
\hline 19 & 6 & 0 & 0.281268 & 2.396632 & -0.005899 \\
\hline
\end{tabular}




\begin{tabular}{|c|c|c|c|c|c|}
\hline 20 & 1 & 0 & -0.350796 & 2.443837 & -0.896001 \\
\hline 21 & 1 & 0 & -0.342340 & 2.458971 & 0.889324 \\
\hline 22 & 1 & 0 & 0.968335 & 3.243225 & -0.016309 \\
\hline
\end{tabular}

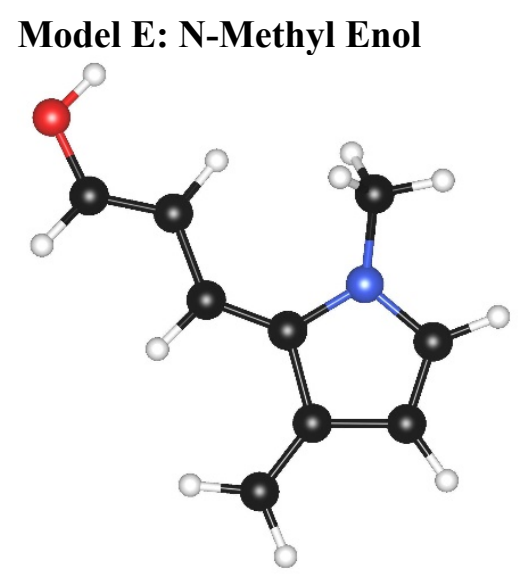

HF $(M 062 X / 6-31+G(d, p))=-479.2919562$ Hartrees

Imaginary Frequencies: none found

Zero-point correction $=0.182499$ (Hartree/Particle)

Thermal correction $=0.145346$ Hartrees

Coordinates from last standard orientation:

\begin{tabular}{|c|c|c|c|c|c|}
\hline \multirow{2}{*}{$\begin{array}{l}\text { Center } \\
\text { Number }\end{array}$} & \multirow{2}{*}{$\begin{array}{l}\text { Atomic } \\
\text { Number }\end{array}$} & \multirow{2}{*}{$\begin{array}{c}\text { Atomic } \\
\text { Type }\end{array}$} & \multicolumn{3}{|c|}{ Coordinates (Angstroms) } \\
\hline & & & $x$ & $Y$ & Z \\
\hline 1 & 6 & 0 & -0.650722 & -0.670247 & 0.052774 \\
\hline 2 & 6 & 0 & -1.922200 & 0.017341 & -0.071012 \\
\hline 3 & 6 & 0 & -3.088221 & -0.635274 & 0.069235 \\
\hline 4 & 1 & 0 & -3.140505 & -1.695965 & 0.296045 \\
\hline 5 & 1 & 0 & -1.950775 & 1.077018 & -0.309168 \\
\hline 6 & 1 & 0 & -0.725667 & -1.743903 & 0.211245 \\
\hline 7 & 6 & 0 & 0.615579 & -0.200784 & -0.028870 \\
\hline 8 & 6 & 0 & 1.852053 & -1.037689 & 0.010848 \\
\hline 9 & 6 & 0 & 2.440072 & 1.149802 & -0.069816 \\
\hline 10 & 1 & 0 & 2.949845 & 2.105021 & -0.101534 \\
\hline 11 & 6 & 0 & 1.927801 & -2.380743 & 0.001774 \\
\hline 12 & 1 & 0 & 2.891954 & -2.876468 & 0.024558 \\
\hline 13 & 1 & 0 & 1.045746 & -3.010647 & -0.032984 \\
\hline 14 & 6 & 0 & 2.964145 & -0.088152 & 0.011319 \\
\hline 15 & 1 & 0 & 4.012730 & -0.344923 & 0.033200 \\
\hline 16 & 8 & 0 & -4.329156 & -0.096408 & -0.046533 \\
\hline 17 & 1 & 0 & -4.263114 & 0.843554 & -0.254789 \\
\hline 18 & 7 & 0 & 1.051622 & 1.131192 & -0.146231 \\
\hline 19 & 6 & 0 & 0.246720 & 2.292357 & 0.158068 \\
\hline 20 & 1 & 0 & -0.437642 & 2.536068 & -0.660513 \\
\hline 21 & 1 & 0 & -0.332165 & 2.136945 & 1.076987 \\
\hline 22 & 1 & 0 & 0.910124 & 3.146549 & 0.306919 \\
\hline
\end{tabular}




\section{Model E: N-Methyl Iminium}

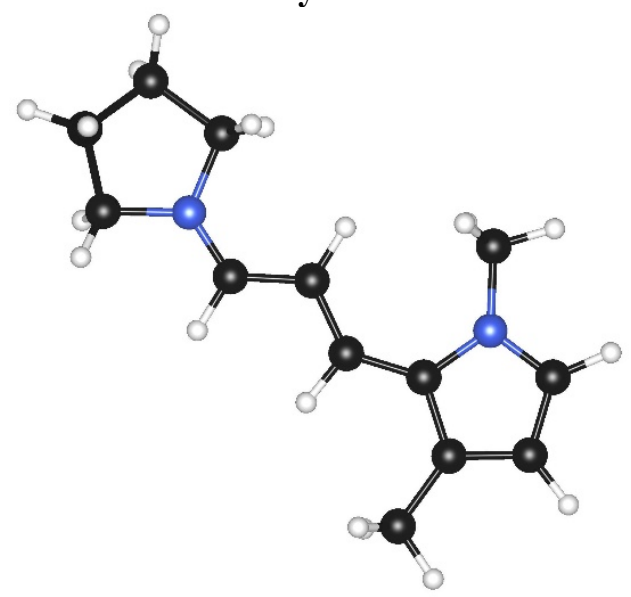

HF $(M 062 X / 6-31+G(d, p))=-615.8522084$ Hartrees

Imaginary Frequencies: none found

Zero-point correction $=0.303822$ (Hartree/Particle)

Thermal correction $=0.261010$ Hartrees

Coordinates from last standard orientation:

\begin{tabular}{|c|c|c|c|c|c|}
\hline \multirow{2}{*}{$\begin{array}{l}\text { Center } \\
\text { Number }\end{array}$} & \multirow{2}{*}{$\begin{array}{l}\text { Atomic } \\
\text { Number }\end{array}$} & \multirow{2}{*}{$\begin{array}{l}\text { Atomic } \\
\text { Type }\end{array}$} & \multicolumn{3}{|c|}{ Coordinates (Angstroms) } \\
\hline & & & $\mathrm{X}$ & Y & Z \\
\hline & & & -- & --1 & ---------- \\
\hline 1 & 6 & 0 & 0.912313 & -0.629013 & -0.029915 \\
\hline 2 & 6 & 0 & -0.325470 & -0.003636 & -0.043120 \\
\hline 3 & 6 & 0 & -1.474008 & -0.811759 & -0.064539 \\
\hline 4 & 1 & 0 & -1.352810 & -1.894994 & -0.069582 \\
\hline 5 & 7 & 0 & -2.712684 & -0.386486 & -0.074261 \\
\hline 6 & 6 & 0 & -3.894254 & -1.276767 & -0.071687 \\
\hline 7 & 1 & 0 & -3.708748 & -2.137617 & 0.573559 \\
\hline 8 & 1 & 0 & -4.079265 & -1.624277 & -1.094441 \\
\hline 9 & 6 & 0 & -5.011037 & -0.355596 & 0.412519 \\
\hline 10 & 1 & 0 & -4.997057 & -0.291063 & 1.505092 \\
\hline 11 & 1 & 0 & -5.995407 & -0.706767 & 0.100694 \\
\hline 12 & 6 & 0 & -4.628334 & 0.991971 & -0.210827 \\
\hline 13 & 1 & 0 & -5.111085 & 1.841619 & 0.273405 \\
\hline 14 & 1 & 0 & -4.896119 & 1.007262 & -1.271654 \\
\hline 15 & 6 & 0 & -3.105716 & 1.036007 & -0.055372 \\
\hline 16 & 1 & 0 & -2.600691 & 1.571798 & -0.863850 \\
\hline 17 & 1 & 0 & -2.800613 & 1.469582 & 0.904512 \\
\hline 18 & 1 & 0 & -0.436240 & 1.072017 & -0.032773 \\
\hline 19 & 1 & 0 & 0.889204 & -1.719459 & -0.043623 \\
\hline 20 & 6 & 0 & 2.217478 & -0.130222 & -0.002254 \\
\hline 21 & 6 & 0 & 3.395097 & -0.929313 & 0.005450 \\
\hline 22 & 6 & 0 & 3.969423 & 1.239841 & 0.049360 \\
\hline 23 & 1 & 0 & 4.494198 & 2.185933 & 0.074371 \\
\hline 24 & 6 & 0 & 3.445057 & -2.425155 & -0.020992 \\
\hline 25 & 1 & 0 & 4.480479 & -2.765610 & 0.022718 \\
\hline 26 & 1 & 0 & 2.915427 & -2.861089 & 0.831572 \\
\hline 27 & 6 & 0 & 4.480217 & -0.059878 & 0.037908 \\
\hline 28 & 1 & 0 & 5.527207 & -0.325807 & 0.051723 \\
\hline 29 & 1 & 0 & 3.002345 & -2.824303 & -0.938821 \\
\hline 30 & 7 & 0 & 2.627456 & 1.205963 & 0.025185 \\
\hline
\end{tabular}




\begin{tabular}{rrrrrr}
31 & 6 & 0 & 1.776571 & 2.385237 & 0.030739 \\
32 & 1 & 0 & 1.168176 & 2.419166 & -0.875949 \\
33 & 1 & 0 & 1.138276 & 2.389356 & 0.917260 \\
34 & 1 & 0 & 2.415293 & 3.267603 & 0.055697 \\
\hline
\end{tabular}

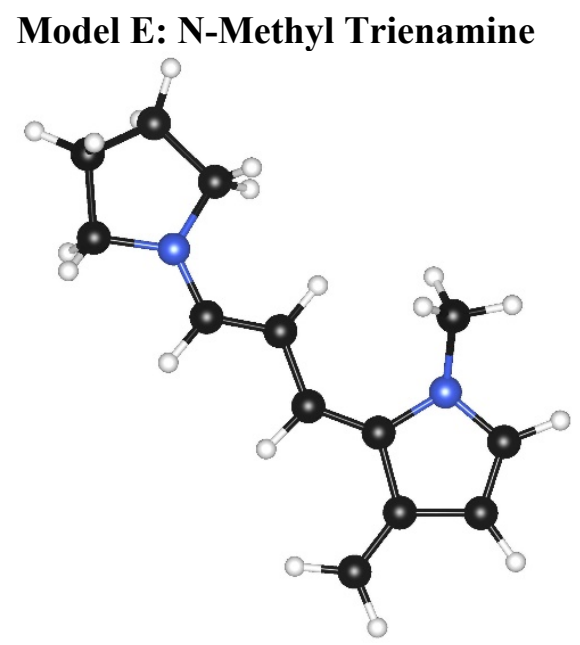

HF $(M 062 X / 6-31+G(d, p))=-615.4064184$ Hartrees

Imaginary Frequencies: none found

Zero-point correction $=0.288375$ (Hartree/Particle)

Thermal correction $=0.244371$ Hartrees

Coordinates from last standard orientation:

\begin{tabular}{|c|c|c|c|c|c|}
\hline \multirow{2}{*}{$\begin{array}{l}\text { Center } \\
\text { Number }\end{array}$} & \multirow{2}{*}{$\begin{array}{l}\text { Atomic } \\
\text { Number }\end{array}$} & \multirow{2}{*}{$\begin{array}{c}\text { Atomic } \\
\text { Type }\end{array}$} & \multicolumn{3}{|c|}{ Coordinates (Angstroms) } \\
\hline & & & $X$ & Y & Z \\
\hline & & & 0 & --------1 & $u^{-1}-----1$ \\
\hline 1 & 6 & 0 & 0.983478 & -0.749614 & 0.055383 \\
\hline 2 & 6 & 0 & -0.319601 & -0.129492 & -0.019641 \\
\hline 3 & 6 & 0 & -1.453539 & -0.867583 & 0.114654 \\
\hline 4 & 1 & 0 & -1.378514 & -1.939621 & 0.293732 \\
\hline 5 & 7 & 0 & -2.730011 & -0.396515 & 0.067125 \\
\hline 6 & 6 & 0 & -3.909902 & -1.248274 & 0.090688 \\
\hline 7 & 1 & 0 & -3.823395 & -2.003320 & 0.878825 \\
\hline 8 & 1 & 0 & -4.052955 & -1.768045 & -0.870443 \\
\hline 9 & 6 & 0 & -5.036554 & -0.245236 & 0.338516 \\
\hline 10 & 1 & 0 & -5.094092 & -0.013861 & 1.407378 \\
\hline 11 & 1 & 0 & -6.010725 & -0.614410 & 0.010488 \\
\hline 12 & 6 & 0 & -4.562747 & 0.985403 & -0.446422 \\
\hline 13 & 1 & 0 & -5.035148 & 1.913737 & -0.118316 \\
\hline 14 & 1 & 0 & -4.783825 & 0.851722 & -1.510614 \\
\hline 15 & 6 & 0 & -3.042462 & 0.991100 & -0.229955 \\
\hline 16 & 1 & 0 & -2.497462 & 1.330742 & -1.121794 \\
\hline 17 & 1 & 0 & -2.741321 & 1.635789 & 0.609516 \\
\hline 18 & 1 & 0 & -0.406193 & 0.934340 & -0.203730 \\
\hline 19 & 1 & 0 & 0.971560 & -1.832115 & 0.173727 \\
\hline 20 & 6 & 0 & 2.224807 & -0.214390 & -0.029231 \\
\hline 21 & 6 & 0 & 3.501059 & -0.982132 & -0.053912 \\
\hline 22 & 6 & 0 & 3.967503 & 1.238380 & -0.046119 \\
\hline 23 & 1 & 0 & 4.426997 & 2.219569 & -0.040646 \\
\hline
\end{tabular}




$\begin{array}{rrrrrr}24 & 6 & 0 & 3.655318 & -2.318715 & -0.128969 \\ 25 & 1 & 0 & 4.647147 & -2.756392 & -0.151491 \\ 26 & 1 & 0 & 2.810611 & -2.997097 & -0.176384 \\ 27 & 6 & 0 & 4.558994 & 0.026925 & -0.032513 \\ 28 & 1 & 0 & 5.620291 & -0.172436 & -0.046607 \\ 29 & 7 & 0 & 2.582712 & 1.150797 & -0.100874 \\ 30 & 6 & 0 & 1.735167 & 2.236674 & 0.341226 \\ 31 & 1 & 0 & 0.987170 & 2.498917 & -0.412855 \\ 32 & 1 & 0 & 1.223076 & 1.978317 & 1.276572 \\ 33 & 1 & 0 & 2.358735 & 3.115927 & 0.516642 \\ - & & & & & \end{array}$

